\title{
Computations of form and stability of rotating drops with finite elements
}

\author{
Berechnungen von Form und Stabilität rotierender Tropfen \\ mit finiten Elementen
}

Von der Fakultät für Mathematik, Informatik und Naturwissenschaften der Rheinisch-Westfälischen Technischen Hochschule Aachen zur Erlangung des akademischen Grades eines Doktors der Naturwissenschaften genehmigte Dissertation

\author{
vorgelegt von \\ Diplom-Mathematiker Claus-Justus Heine \\ aus Krefeld. \\ Berichter: Universitätsprofessor Dr. Josef Bemelmans \\ Universitätsprofessor Dr. Gerhard Dziuk
}

Tag der mündlichen Prüfung: 15. Dezember 2003

Diese Dissertation ist auf den Internetseiten der Hochschulbibliothek online verfügbar. 

At this point I would like to thank Prof. Josef Bemelmans for proposing this problem to me and for his excellent supervision. I would like to thank Prof. Gerhard Dziuk for introducing the finite element theory to me, for providing suitable literature and his help concerning the discretisation of curvature related problems. Further, I would like to thank Alfred Schmidt and Kunibert Siebert for their finite element toolbox ALBERT and for answering a bunch of beginner's questions concerning finite elements. 


\begin{abstract}
We consider the problem of a drop rotating rigidly at a fixed angular velocity. The centrifugal forces are balanced by surface tension alone. Such a drop is described by the Young-Laplace equation:

$$
2 H=4 r^{2} \omega^{2}+C, \quad \int_{\Omega} d x=\text { const. }
$$

Here $H$ denotes the mean curvature, $r$ the orthogonal distance to the axis of rotation, $C$ the Lagrange multiplier for the volume constraint, $\Omega \subset \mathbb{R}^{3}$ the region enclosed by the drop surface and $\omega$ the angular velocity.

Subject of this work is the numerical computation of the bifurcation of shape families from explicitly known axisymmetric drops. The bifurcation parameter is $\omega$.

This problem has been treated numerically by R.A. Brown and L.E. Scriven in 1980 [Brown and SCRIVEn 1980a]. We present an algorithm which avoids an explicit global parametrisation of the drop surface and does not impose a meridional reflective symmetry on the drop shapes, using ideas introduced by G. Dziuk for computing evolutionary surfaces [DZIUK 1991].

The results of the numerical experiments extend the results formerly found by Brown and Scriven and reveal several new branches of spheroidal drop shapes. Furthermore drop shapes of annular type have been computed branching from an axisymmetric family of tori which was found by R. Gulliver [Gulliver 1984].
\end{abstract}




\section{Zusammenfassung}

Wir betrachten einen Tropfen, der als starrer Körper mit konstanter Winkelgeschwindigkeit rotiert. Dabei steht die Zentrifugalkraft im Gleichgewicht mit der Oberflächenspannung; andere Kräfte werden nicht berücksichtigt. Solch ein Tropfen wird von der Young-Laplace Gleichung beschrieben:

$$
2 H=4 r^{2} \omega^{2}+C, \quad \int_{\Omega} d x=\text { const. }
$$

Dabei bezeichnet $H$ die mittlere Krümmung, $r$ den Abstand von der Rotationsachse, $C$ den Lagrangeschen Multiplikator für die Volumenbedingung, $\Omega \subset \mathbb{R}^{3}$ das Gebiet, das der Tropfen einnimmt und $\omega$ die Winkelgeschwindigkeit.

Der Gegenstand der Untersuchung ist die numerische Berechnung von Familien von Tropfen, die von explizit bekannten zylindersymmetrischen Familien abzweigen. Der Verzweigungsparameter ist $\omega$.

Dieses Problem wurde bereits 1980 von R.A. Brown und L.A. Scriven numerisch behandelt [Brown and SCRIVEn 1980a]. Wir stellen einen Algorithmus vor, der eine explizite globale Parametrisierung der Oberfläche des Tropfens vermeidet und ohne die Annahme einer meridialen Spiegelsymmetrie auskommt. Benutzt werden dazu Ideen von G. Dziuk zur Berechnung von Flächen, die sich unter dem Krümmungsfluß entwickeln [DZIUK 1991].

Die Ergebnisse der numerischen Experimente erweitern die Resultate von Brown und Scriven und zeigen etliche neue Verzweigungen. Außerdem wurden Tropfen vom Typ eines Torus berechnet, welche von einer zylindersymmetrischen Familie abzweigen, die von R. Gulliver gefunden wurde [GuLLIVER 1984]. 



\section{Contents}

1 Introduction 1

1.1 The physical model . . . . . . . . . . . . . . . . . 2

1.2 Historical Overview . . . . . . . . . . . . . . . . . 4

1.3 Notation and Fundamental Definitions . . . . . . . . . . . . . . . 6

1.3.1 Analysis in $\mathbb{R}^{n} \ldots \ldots \ldots \ldots$

1.3.2 Differential geometry . . . . . . . . . . . . . . 9

1.3.3 Some aspects from bifurcation theory . . . . . . . . . . . . . 14

1.4 Exact solutions . . . . . . . . . . . . . . . . . 16

1.4.1 Solutions of spheroidal type . . . . . . . . . . . . . 17

1.4.2 Solutions of annular type . . . . . . . . . . . . . . 19

2 The path tracking problem $\quad 21$

2.1 First and second variation of the energy functional . . . . . . . . . 22

2.1.1 The first variation of $E(\Gamma, C) \ldots \ldots . \ldots 22$

2.1.2 The second variation of $E(\Gamma, C) \ldots \ldots . \ldots 24$

2.2 The linear Euler-Lagrange equations . . . . . . . . . . . . . . . . . 28

2.2 .1 Lie-symmetries . . . . . . . . . . . . . . . . . . 29

2.2.2 Weak solutions of the linearisation . . . . . . . . . . 32

2.3 The continuous Newton iteration . . . . . . . . . . . . 37

2.3.1 The differential operators . . . . . . . . . . . . 38

2.3.2 The iteration step . . . . . . . . . . . . . . . . 40

2.4 The discretised Newton iteration _ . . . . . . . . . . . . . . . 42

2.4.1 Iso-parametric triangulations . . . . . . . . . . . . . . . . . 42

2.4 .2 Iso-parametric finite elements . . . . . . . . . . . . . . 47 
2.4.3 A finite element formulation of the Newton iteration . . . . . . 48

2.4.4 An error indicator . . . . . . . . . . . . . . . . . . 52

2.5 The solving of the bifurcation equation . . . . . . . . . . . 53

2.5.1 Finding of bifurcation points . . . . . . . . . . . . . 53

2.5.2 The discretisation of the bifurcation equation . . . . . . . . 55

2.5.3 Solvers for the constraint eigen-problem . . . . . . . . . . 55

2.6 The discrete path-tracking algorithm . . . . . . . . . . . . 56

3 Numerical methods for iso-parametric meshes $\quad 59$

3.1 Approximate normals . . . . . . . . . . . . . . . . . . . . . 59

3.2 Curvature for Discrete Surfaces . . . . . . . . . . . . . . . . . . 61

3.2.1 Definition of the Discrete Curvature . . . . . . . . . . . . 61

3.2 .2 Convergence tests . . . . . . . . . . . . . . 63

3.3 Local refinement and coarsening . . . . . . . . . . . . . . . . . . . 69

3.4 Mesh smoothing . . . . . . . . . . . . . . . . . . . . 70

3.4.1 Subdivision of obtuse angles . . . . . . . . . . . . . . 70

3.4.2 Vertex displacement . . . . . . . . . . . . . . . 72

3.4.3 Smoothing functionals . . . . . . . . . . . . . . . 77

3.4.4 Projection to discretised surfaces . . . . . . . . . . . . . 88

$\begin{array}{lll}4 & \text { Numerical results } & 93\end{array}$

4.1 Experimental convergence tests . . . . . . . . . . . . . . . . 93

4.1.1 Convergence of the discrete Newton iteration . . . . . . . . . . . 93

4.1.2 Accuracy of the error indicators . . . . . . . . . . . . . 95

4.1 .3 Eigenvalue computation . . . . . . . . . . . . . . . 97

4.2 Bifurcation diagrams and drop-shapes . . . . . . . . . . . . . . . 100

4.2.1 Two-lobed spheroidal shapes . . . . . . . . . . . . . . . . . . . 102

4.2.2 Three- and four-lobed shapes . . . . . . . . . . . . . . . 108

4.2.3 Five- and six-lobed spheroidal shapes . . . . . . . . . . . . . . . 110

4.2.4 Annular drop shapes . . . . . . . . . . . . . . . . . . . . . . . 114

$\begin{array}{ll}\text { Bibliography } & 115\end{array}$

$\begin{array}{ll}\text { Index of Notations } & 119\end{array}$ 


\section{Algorithms, figures and tables}

\section{List of Algorithms}

2.3.1 A modified Newton iteration . . . . . . . . . . . . . . . . 38

2.3.2 The iteration step of the continuous Newton iteration . . . . . . . . . . 40

2.4.1 The iteration step of the discrete Newton iteration . . . . . . . . . . . . 49

2.5.1 Flow char: finding of branch-points . . . . . . . . . . . . . . 54

2.6.1 Flow chart: adaptive computation of drop-shapes . . . . . . . . . 58

3.3.1 Local refinement of a triangular mesh . . . . . . . . . . . . . . . . . 69

3.4.1 Mesh adaption and subdivision of obtuse angles . . . . . . . . . . 72

3.4.2 Outline of combined averaging and optimisation based smoothing . . . . 73

3.4.3 CG method for a smoothing-functional . . . . . . . . . . . . . 75

3.4.4 Optimisation-Based Mesh-Smoothing Algorithm . . . . . . . . . . . 76

3.4 .5 Projection of a node $P$ to $\Gamma_{h, 0} \ldots \ldots \ldots \ldots$. . . . . . . 88

\section{List of Figures}

1.1 Plateau's apparatus and drop shapes. . . . . . . . . . . . . 4

1.2 T.G. Wang's repetition of Plateau's experiments. . . . . . . . . . . 5

1.3 Development of a freely rotating two-lobed drop, USML2 space-lab flight

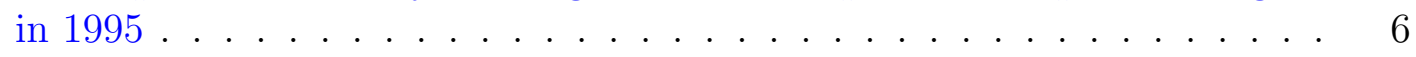

1.4 Limit surfaces of annular and simply connected family . . . . . . . . 16

$1.5 \omega$-L phase diagram for exact annular and simply connected solutions. . . 17

1.6 Computation of the spheroidal drop shapes. . . . . . . . . . . . . 18

1.7 Meridional sections through some spheroidal shapes at different values of $\omega 19$ 
1.8 Meridional sections through some annular shapes at different values of $\omega^{2} .19$

2.1 The action of an infinitesimal rotation f . . . . . . . . . . . . 31

2.2 Artificial meridional reflective symmetry . . . . . . . . . . . . . . 31

2.3 Displacement in normal direction during the Newton iteration . . . . . . 41

2.4 Failing polar coordinate system . . . . . . . . . . . . . . 42

2.5 Parametrisation with Lagrange finite elements . . . . . . . . . . . . 45

3.1 Convergence tests for the discrete curvature . . . . . . . . . . . . 63

3.2 Refinement and coarsening of a conforming patch . . . . . . . . . . 69

3.3 Refinement of an iso-parametric element . . . . . . . . . . . . . 70

3.4 Two-lobed drop with obtuse angles . . . . . . . . . . . . . . . 71

3.5 Examples for subdivision of large angles . . . . . . . . . . . . . . 71

3.6 Contour plots of the smoothing-functionals . . . . . . . . . . . . . . 80

3.7 Contour plots of smoothing-functionals, distorted patch . . . . . . . . . 81

3.8 Planar test triangulation . . . . . . . . . . . . . . . . . . . 83

3.9 Distribution of angles with different smoothing-functionals . . . . . . . . 84

3.10 Magnified parts of Figure 3.9 on page $84 \ldots \ldots$. . . . . . . . . 85

3.11 Mesh-smoothing for a parametric example mesh . . . . . . . . . . . . . . 85

3.12 Geometry and smoothed meshes for a parametric example . . . . . . . 86

3.13 Different smoothing-functionals acting on the two-lobed family . . . . . . 87

3.14 Result of path-tracking using optimisation based mesh-smoothing . . . . 87

3.15 Projection to triangulations . . . . . . . . . . . . . . 89

3.16 Projection to discrete surfaces . . . . . . . . . . . . . . . . . . . 90

3.17 Projection to parametric surfaces . . . . . . . . . . . . . . . . 91

4.1 Drop shapes used during the EOC-tests . . . . . . . . . . . . . . . . . . 95

4.2 EOC for $\mathrm{L}_{2^{-}}$and $\mathrm{H}^{1}$-errors while tracking the spheroidal family . . . . . 96

4.3 The first five eigenvalues, computed for some of the shapes used for the experimental convergence tests shown on the pages before . . . . . . . 97

$4.4 \mathrm{~L}_{2}$-errors and error-indicators while tracking the spheroidal family . . . . 98

$4.5 \mathrm{H}^{1}$-errors and error-indicators during path-tracking . . . . . . . . . . 99

4.6 Bifurcation diagram by Brown and Scriven . . . . . . . . . . . . . 100 
4.7 Bifurcation diagram resulting from Algorithm 2.6.1 on page 58 . . . . . 101

4.8 Spectrum of the linearised Euler-Lagrange equations for the axially symmetric spheroidal family after the turning point . . . . . . . . . . . . . 102

4.9 Two-lobed drop shapes . . . . . . . . . . . . . . . . . 103

4.10 Spectrum of the linearization, two-lobed branch . . . . . . . . . . . . . 104

4.11 Sub-branch of the two-lobed family branching at $\omega \approx 0.262 \ldots \ldots 5$

4.12 Two-lobed sub-branch $(1) \ldots \ldots \ldots$. . . . . . . . . . . 106

4.13 Two-lobed sub-branch $(2) \ldots \ldots \ldots$. . . . . . . . . . . 107

4.14 Three-lobed branch at $\omega=0.1056$. . . . . . . . . . . . . . . 108

4.15 Three-lobed sub-branch breaking the meridional reflective symmetry . . . 109

4.16 "Winding-up" of the two-, five- and six-lobed families . . . . . . . . . . . 110

4.17 Five-lobed drop shapes . . . . . . . . . . . . . . . . . . 111

4.18 Five-lobed shapes after and before "winding-up" in the $(\omega, L)$-plane . . . 112

4.19 Six-lobed shapes _. . . . . . . . . . . . . . . . . . . 113

4.20 Bifurcations from the axially symmetric family of annular shapes . . . . . 114

\section{List of Tables}

3.1 Discrete curvature, problem 1, relative errors . . . . . . . . . . 65

3.2 Discrete curvature, problem 2, relative errors . . . . . . . . . . . . 66

3.3 Discrete curvature, problem 3, relative errors . . . . . . . . . . . . . . 67

3.4 Discrete curvature, problem 4, relative errors . . . . . . . . . . . 68

3.5 Extremal angles for a planar triangulation . . . . . . . . . . . . . 83

4.1 Errors and EOCs for a spheroidal drop shape at $\omega \approx 0.7138 \ldots \ldots$. . . . 94

4.2 Errors and EOCs for an annular drop shape at $\omega \approx 0.7069 \ldots \ldots 4$ 



\section{Chapter 1}

\section{Introduction}

This work is concerned with the numerical computation of the equilibrium figures of rotating drops subject to surface tension and centrifugal forces alone.

Such drops were already studied by J.A.F. Plateau in the first half of the nineteenth century. He made experiments with drops immersed in a tank of liquid of almost the same density as the drop and used a wooden shaft to turn the drops at constant angular velocity. Newer experiments were performed in the context of the low gravity fluid dynamics experiments of the Spacelab in the eightieth and ninetieth of the $20^{\text {th }}$ century. This time, an acoustic multi-pole field was used to initiate the rotation of the drops.

The physical experiments and analytical and numerical works performed in the (roughly) 160 years following Plateau's experiments suggest that the equations governing this problem show an interesting bifurcation behaviour with a rich variety of solution families. However, no strict analytical proof exists yet for the existence of any of the bifurcation phenomena observed by either physical or numerical experiments. The attempt to give a short history of related works is made below in Section 1.2.

In this work we describe a numerical method which uses parametric finite elements on the surface of the drop and, in addition, uses a local adaptive refinement strategy and methods to counter-balance the degeneration of the surface mesh.

This enables us to compute geometrically complicated surfaces without running into coordinate singularities and to handle multiply-connected surfaces like annuli.

Furthermore, for the first time in the numerical treatment of this problem no artificial symmetries are imposed on the drop shapes. Thus our path-tracking algorithm is able to compute a much larger part of the solution-set than it was possible to compute with previous numerical method. Particularly we found several solution-branches not known before.

The remainder of this work is structured as follows:

First we present the physical problem with the corresponding mathematical model and give a short historical overview. After introducing the necessary definitions and notations we present the exact solutions known presently. 
In the second part of this work we explain the discretisation of the differential equation describing the drops and the path-tracking algorithm. The numerical methods for the surface mesh are described in detail in the third chapter. Finally, the last chapter presents the results of the numerical experiments.

The implementation of our algorithm for the computation of the drop-shapes (see Algorithm 2.6.1 on page 58) was based on version 0.2 of the finite element toolbox ALBERT [SChmidT and Siebert 1998]. The toolbox ARPACK [LEHOuCQ et al. 1998] was used as back-end for the eigenvalue computations needed for solving the bifurcation equations.

\subsection{The physical model}

We consider a rotating drop held together by its surface tension alone. Gravitational forces are neglected.

The drop rotates as a rigid body with constant angular velocity $\omega$ around the $z$-axis. The surface tension is proportional to the mean curvature with a constant factor $\sigma$. Moreover, the massdensity $\rho$ of the drop is constant.

The aim is to compute the shape of the drop in dependence on $\omega$ under the constraint of a prescribed volume $V$. So we are searching for compact embedded surfaces $\Gamma \subset \mathbb{R}^{3}$ which satisfy the differential equation derived from the physical model described above.

Let $\Omega \subset \mathbb{R}^{3}$ be the region enclosed by $\Gamma$. Then the drop's energy $E$ in its restsystem is given by

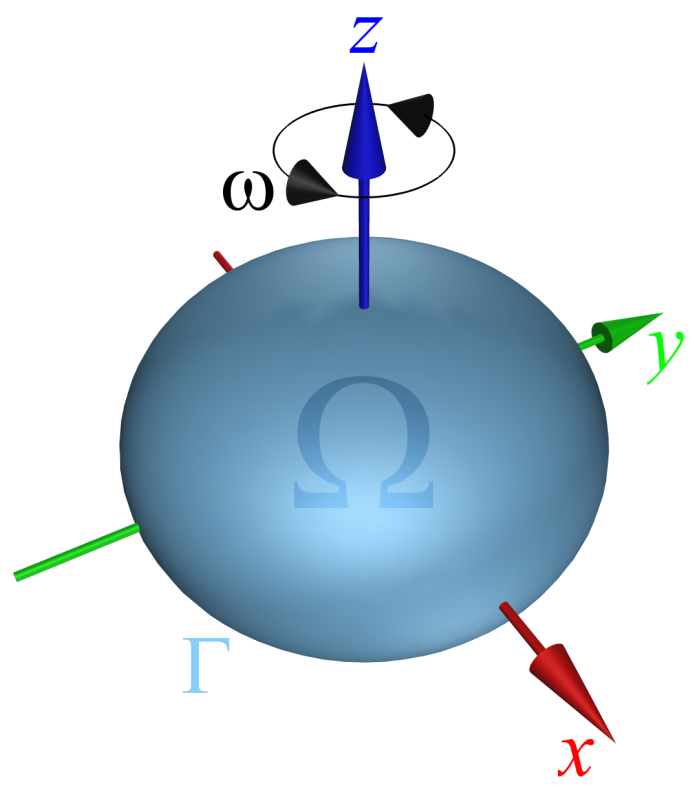

$$
E=E_{\text {surf }}+E_{\text {rot }}-C(|\Omega|-V),
$$

with the surface energy $E_{\text {surf }}:=\sigma|\Gamma|$, the rotational energy $\omega^{2} \frac{\rho}{2} \int_{\Omega} r^{2} d x$ and the Lagrange-parameter $C$ accounting for the volume-constraint. $r$ denotes the distance from the axis of rotation. The drop rotates around the $z$ - or $x_{3}$-axis where a point $P \in \mathbb{R}^{3}$ has the components $(x, y, z) \equiv\left(x_{1}, x_{2}, x_{3}\right)$.

Without loss of generality (see the remark at the end of this section on page 4) we can assume that $V=\frac{4}{3} \pi$. Division of $E$ by $\sigma$ then yields the normalised energy

$$
\hat{E}:=\frac{1}{\sigma} E=|\Gamma|-\frac{1}{2} \hat{\omega}^{2} \frac{\rho}{\sigma} \int_{\Omega} r^{2} d x-\bar{C}\left(|\Omega|-\frac{4}{3} \pi\right), \text { with } \bar{C}:=\frac{\hat{C}}{\sigma},
$$

Substituting $\bar{\omega}^{2}:=\frac{\rho}{8 \sigma} \hat{\omega}^{2}$ one gets rid of the constants $\rho$ and $\sigma$. So the only parameter left is the normalised angular velocity $\bar{\omega}$ :

$$
\bar{E}=|\Gamma|-4 \bar{\omega}^{2} \bar{M}-\bar{C}\left(|\Omega|-\frac{4}{3} \pi\right)
$$


where $\bar{M}:=\int_{\Omega} r^{2} d x$ is the normalised moment of inertia.

In the following we are only concerned with the normalised energy of equation (1.1) and so we omit the bars. For the remainder $E, \omega, M$ and $C$ each denote the quantities $\bar{E}$, $\bar{\omega}, \bar{M}$ and $\bar{C}$ of equation (1.1).

The energy functional $E$ describes a drop rotating at constant angular velocity $\omega$. However, the natural parameter for a freely rotating drop would be its angular momentum. Such a drop is described by another functional $R$ (the so called "Routhian") which is related to $E$ by a Legendre-transformation (see any standard text-book for theoretical mechanics, for example [LANDAu and Lifschitz 1990, Chapter VII]).

$$
R:=E-\omega \frac{\partial E}{\partial \omega}=|\Gamma|+8 \frac{L^{2}}{2 M}-C\left(|\Omega|-\frac{4}{3} \pi\right) .
$$

The formal first and second variation of $E$ and $R$ are given by:

\begin{tabular}{|c|c|}
\hline Preserved angular velocity $\omega$ & Preserved angular momentum $L$ \\
\hline$E=|\Gamma|-4 \omega^{2} M-C\left(|\Omega|-\frac{4}{3} \pi\right)$ & $R=|\Gamma|+4 \frac{L^{2}}{M}-C\left(|\Omega|-\frac{4}{3} \pi\right)$ \\
$\delta E=\delta|\Gamma|-4 \omega^{2} \delta M-C \delta|\Omega|$ & $\delta R=\delta|\Gamma|-4 \frac{L^{2}}{M^{2}} \delta M-C \delta|\Omega|$ \\
$\delta^{2} E=\delta^{2}|\Gamma|-4 \omega^{2} \delta^{2} M-C \delta^{2}|\Omega|$ & $\delta^{2} R=\delta^{2}|\Gamma|-4 \frac{L^{2}}{M^{2}} \delta^{2} M-C \delta^{2}|\Omega|$ \\
& $+8 \frac{L^{2}}{M^{3}}(\delta M)^{2}$ \\
\hline
\end{tabular}

Because of $\omega^{2}=L^{2} / M^{2}$ it is clear that $E$ and $R$ have the same stationary points. Obviously, a rigid body rotating at constant angular velocity has a constant angular momentum and vice versa.

However, in the second variation of $R$ there is the additional term $8 \frac{L^{2}}{M^{3}}(\delta M)^{2} \geq 0$. So there may be stationary points which are minima of $R$ but not of $E$. The reason is that the conservation of angular momentum is an additional constraint on the admissible perturbations.

Another important difference is that the Euler-Lagrange-equations derived from the variation of $R$ are integro-differential equations. In contrast, the variation of $E$ yields "only" partial differential equations. The reason is that the first variation of $R$ contains the term $M=\int_{\Omega} r^{2} d x$ explicitly while the first variation of $E$ only contains the first variation of $M$.

The Euler-Lagrange equations for the first variation of $E$ are

$$
2 H_{\Gamma} \nu_{\Gamma} \equiv-\Delta_{\Gamma} \mathrm{id}_{\Gamma}=\left(4 \omega^{2} r^{2}+C\right) \nu_{\Gamma}, \quad \int_{\Gamma} \operatorname{id}_{\Gamma} \nu_{\Gamma} d \Gamma=4 \pi
$$

Here $\Delta_{\Gamma}$ denotes the Laplace-Beltrami operator, $\nu$ the outer normal-field and $\operatorname{id}_{\Gamma}: \Gamma \rightarrow \Gamma: x \mapsto x$ the identity mapping on $\Gamma$. Furthermore the volume constraint has been transformed into an integral over the surface $\Gamma$ by virtue of Gauß' formula, using div $\mathrm{id}_{\mathbb{R}^{3}}=3$. 
The unknowns in equation (1.3) above are the surface $\Gamma$ itself and the Lagrangemultiplier $C$ for the volume constraint. $\omega$ enters as a parameter. A brief derivation of the Euler-Lagrange equations is in Section 2.1 on page 22.

We note that by applying a scaling transformation $x \mapsto \lambda \cdot x(\lambda>0)$ a solution $\Gamma$ of the Euler-Lagrange equations (1.3) is transformed into a solution $\Gamma^{\lambda}$ of the Equation

$$
2 H_{\Gamma^{\lambda}} \nu_{\Gamma^{\lambda}} \equiv-\Delta_{\Gamma^{\lambda}} \operatorname{id}_{\Gamma^{\lambda}}=\left(4 \frac{\omega^{2}}{\lambda^{3}} r^{2}+\frac{C}{\lambda}\right) \nu_{\Gamma^{\lambda}} x, \quad \int_{\Gamma^{\lambda}} \operatorname{id}_{\Gamma^{\lambda}} \nu_{\Gamma^{\lambda}} d \Gamma^{\lambda}=4 \pi \lambda^{3} .
$$

This follows easily from the facts that $H_{\Gamma^{\lambda}}=\frac{1}{\lambda} H_{\Gamma}$ and $\nu_{\Gamma^{\lambda}}=\nu_{\Gamma}$.

The goal is now to find bifurcations from the two explicitly known families of solutions to (1.3), which are described in Section 1.4. The angular velocity $\omega$ is used as bifurcation parameter. The path-tracking algorithm and the discretisation of equation (1.3) will be described in Chapter 2 below.

\subsection{Historical Overview}

The following is a brief summary of some analytical and numerical results concerning the computation of shape and stability of equilibrium configurations of rotating drops. The list is - of course - incomplete.

1843 J.A.F. Plateau Plateau made physical experiments with a drop immersed in a tank of liquid of almost the same density as the drop. The apparatus is shown in

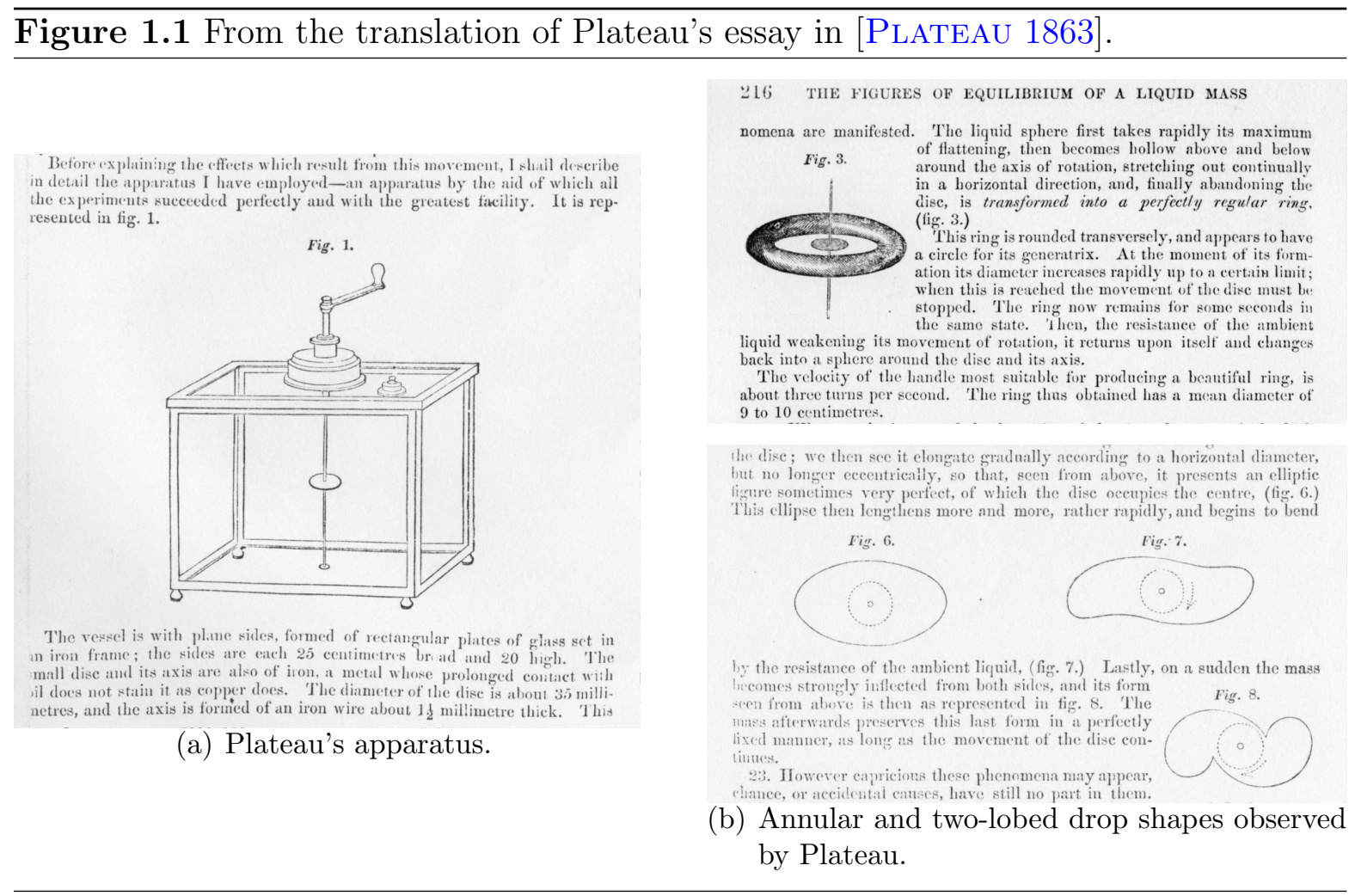


Figure 1.1(a). By turning the vertically mounted shaft the drop could be brought into rotation. Some of the figures observed by Plateau are shown in Figure 1.1(b) on the preceding page. The experiments were published in [Plateau 1843] and later in English translation in [Plateau 1863].

1869 A. Beer He found a family of exact solutions to (1.3). The derivation can be found in his book [BEER 1869], a short outline is given in Section 1.4 below.

1914 Lord Rayleigh By a Taylor-series expansion [RAYLEIGH 1914] found that certain annular equilibrium shapes are stable in the cylinder-symmetric setting.

1965 S. Chandrasekhar He found, by considering oscillations around a linearisation of (1.3), estimates for the value of the angular velocity at which the drop-shapes found by Beer become unstable, see [CHANDRASEKHAR 1965].

1968 L.A. Slobozhanin The annular axisymmetric solutions are actually unstable; [MYSHKis et al. 1987] gives a short outline of Slobozhanin's work, see also [Slobozhanin 1968].

1980 R.A. Brown, L.E. Scriven They performed numerical experiments with a finite element method, investigating bifurcations from the axisymmetric family found by Beer. They found three families of bifurcations - a two-lobed, a threelobed and a four-lobed family - which are no longer cylinder-symmetric, but allow only discrete rotation-groups of order 2,3 and 4 . The results were published in [Brown and SCRIVEn 1980a]. We will comment further on their numerical method below, as our work extends their results.

1982 H.C. Wente He considered stationary rotating drops held together by selfgravitation and surface tension in [WENTE 1982] and showed that every such a drop possesses a reflective symmetry about its equatorial plane.

1984 R. Gulliver He found a (more or less) explicit expression for axisymmetric annular solutions of (1.3), very similar to the representation of the simply connected

Figure 1.2 T.G. Wang's repetition of Plateau's experiments. The pictures are taken from [WANG 1988].

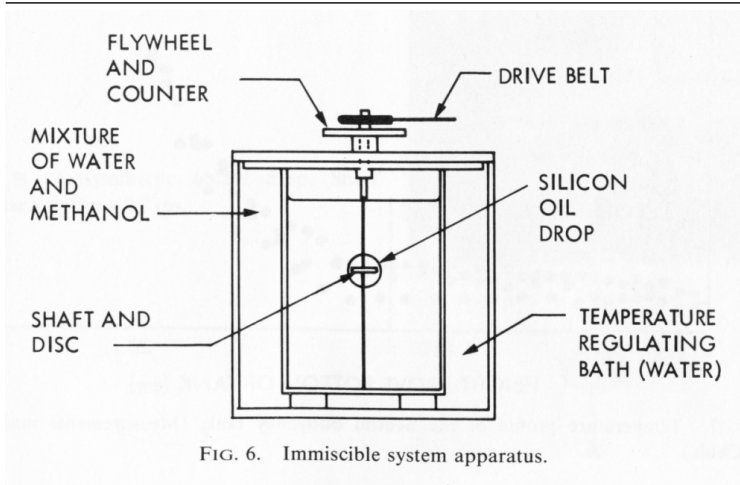

(a) Wang's apparatus

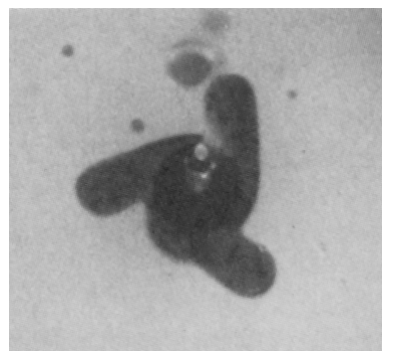

(b) Three-lobed drop shape

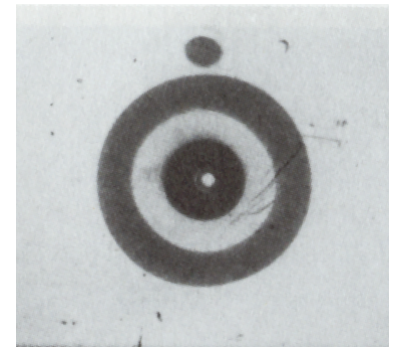

(c) Annular shape 
axisymmetric shapes found by [BEER 1869]. We comment further on those two families in Section 1.4 below.

1985 T.G. Wang Wang repeated and extended Plateau's experiments, using drops of water immersed into a tank of glycerin.

Figure 1.2 on the preceding page shows some of the drop shapes he observed, together with a sketch of the apparatus he has used.

Wang had also a major part in the drop-dynamics experiments of the Spacelab 3 mission in 1985. There, an acoustic multi-pole field was used to initiate the rotation of the centimetre-sized water-drops, see [WANG 1988]. The experiments were continued on subsequent Spacelab flights. Figure 1.3 originates from the USML2 flight in 1995 and shows the development of a freely rotating two-lobed drop.

Figure 1.3 Development of a freely rotating two-lobed drop, USML2 space-lab flight in 1995
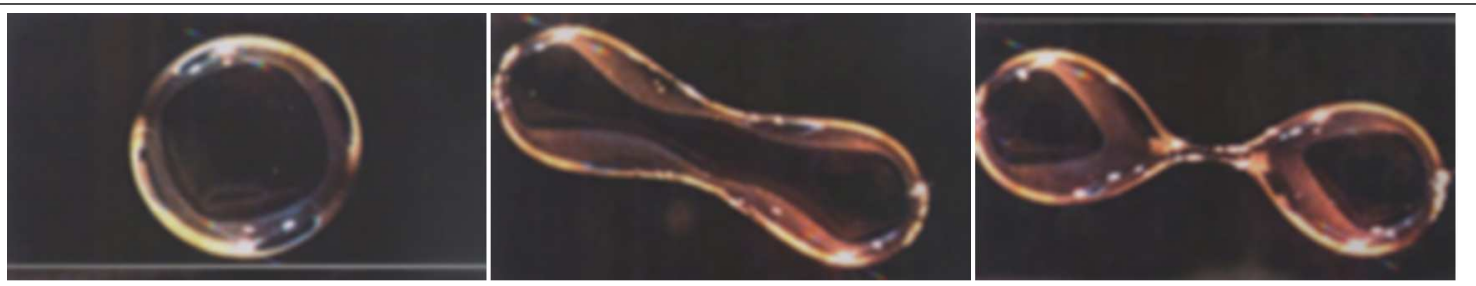

1987 F. Brulois Brulois developed a method to estimate up to arbitrary accuracy the maximum value of the angular velocity $\omega$ up to which the drops of Beer's axisymmetric family of drops are still stable, see [BRULOIS 1987].

\subsection{Notation and Fundamental Definitions}

\subsubsection{Analysis in $\mathbb{R}^{n}$}

\subsubsection{Definition (Natural Numbers, Real Numbers)}

$\mathbb{N}:=\{1,2, \ldots\}$ denotes the set of natural numbers, $\mathbb{N}_{0}:=\mathbb{N} \cup\{0\}$ denotes the set of natural numbers including zero. The set of real numbers is denoted by $\mathbb{R}$.

Further $\mathbb{R}^{+}:=\{x \in \mathbb{R} \mid x>0\}$ and $\mathbb{R}^{\geq 0}, \mathbb{R}^{<0}, \mathbb{R}^{>0} \equiv \mathbb{R}^{+}, \mathbb{R}^{<0}$ denote as usual the sets of non-negative, non-positive, positive and negative real numbers.

\subsubsection{Definition (Scalar Products)}

Let $n, m \in \mathbb{N}$ For $x, y \in \mathbb{R}^{n}$. we denote by $x \cdot y:=\langle x, y\rangle:=x^{T} y$ the Euclidean scalar product. For $A=\left(a_{i j}\right), B=\left(b_{i j}\right) \in \mathbb{R}^{m \times n}$ we denote by $A: B:=\sum_{i=1}^{m} \sum_{j=1}^{n} a_{i j} b_{i j}$, the standard scalar product of $A$ with $B$ in the vector space $\mathbb{R}^{m \times n}$. 
The summation convention is used whenever it is unambiguous: for $a=\left(a_{i}\right), b=\left(b_{i}\right) \in$ $\mathbb{R}^{n}$ we write

$$
a_{i} b_{i}:=a \cdot b=\sum_{i=1}^{n} a_{i} b_{i},
$$

and similarly for other indexed quantities.

\subsubsection{Definition (Polynomial Spaces $P_{k}$ )}

For $k \in \mathbb{N}_{0}$ and $\Omega \subset \mathbb{R}^{n}$ we denote by $P_{k}(\Omega)$ the vector space of polynomials $p: \Omega \rightarrow \mathbb{R}$ of degree less or equal to $k$.

\subsubsection{Definition (Partial Derivatives, Gradients)}

For $\Omega \subset \mathbb{R}^{n}$ open and $f: \Omega \rightarrow \mathbb{R}$ differentiable we denote the partial derivatives of $f$ by

$$
\nabla_{i} f=D_{i} f:=\frac{\partial f}{\partial x_{i}}
$$

and the gradient of $f$ by

$$
\nabla f=D f:=\left(\nabla_{1} f, \ldots, \nabla_{n} f\right)
$$

For $v \in \mathbb{R}^{n}$ we denote by

$$
f_{v}=\frac{\partial f}{\partial v}:=v \cdot \nabla f
$$

the directional derivative of $f$ in direction $v$.

For a multi-index $s=\left(s_{1}, \ldots, s_{n}\right) \in \mathbb{N}_{0}^{n}$ the mixed partial derivatives are denoted by

$$
\nabla^{s} f:=\nabla_{1}^{s_{1}} \cdots \nabla_{n}^{s_{n}} f \text {. }
$$

\subsubsection{Definition (Continuous Function Spaces $C^{k}, C_{0}^{k}$ )}

For $\Omega \subset \mathbb{R}^{n}$ open and bounded we define

$$
\mathrm{C}^{0}(\bar{\Omega}):=\{f: \bar{\Omega} \rightarrow \mathbb{R} \mid f \text { continuous }\}
$$

and for $k \in \mathbb{N}$

$$
\begin{aligned}
\mathrm{C}^{k}(\bar{\Omega}):=\{f: \bar{\Omega} \rightarrow \mathbb{R} \mid & f \text { is } k \text {-times continuously differentiable on } \Omega \\
& \left.\nabla^{s} f \text { can be continuously extended to } \bar{\Omega} \text { for all } s \in \mathbb{N}_{0}^{n},|s| \leq k\right\} .
\end{aligned}
$$

$\mathrm{C}^{0}(\bar{\Omega})$ and $\mathrm{C}^{k}(\bar{\Omega})$ are Banach spaces with the norms

$$
\|f\|_{\sup (\Omega)}:=\sup _{x \in \Omega}|f(x)|, \text { respectively }\|f\|_{\mathrm{C}^{k}(\bar{\Omega})}:=\sum_{|s| \leq k}\left\|\nabla^{s} f\right\|_{\sup (\Omega)} .
$$

Spaces of continuous functions with compact support are denoted by

$$
\mathrm{C}_{0}^{k}(\bar{\Omega}):=\left\{f \in \mathrm{C}^{k}(\bar{\Omega}) \mid \operatorname{supp} f \Subset \Omega\right\} .
$$

Finally

$$
\mathrm{C}^{\infty}(\bar{\Omega}):=\bigcap_{k \in \mathbb{N}} \mathrm{C}^{k}(\bar{\Omega}) \quad \text { and } \quad \mathrm{C}_{0}^{\infty}(\bar{\Omega}):=\bigcap_{k \in \mathbb{N}} \mathrm{C}_{0}^{k}(\bar{\Omega}) .
$$


1.3.6 Definition (HöldeR-SPACES $\mathrm{C}^{k, \alpha}(\bar{\Omega})$, Lipschitz spaCes $\mathrm{C}^{0,1}(\bar{\Omega})$ ) Let $\Omega \subset \mathbb{R}^{n}$ open and bounded and $\alpha \in[0,1], f: \bar{\Omega} \rightarrow \mathbb{R}$. We define

$$
\operatorname{höl}_{\alpha}(f):=\sup _{\substack{x, y \in \bar{\Omega} \\ x \neq y}} \frac{|f(x)-f(y)|}{|x-y|^{\alpha}} .
$$

Then for $k \in \mathbb{N}_{0}$ we define Banach-spaces

$$
\mathrm{C}^{k, \alpha}(\bar{\Omega}):=\left\{f \in \mathrm{C}^{k}(\bar{\Omega}) \mid \operatorname{höl}_{\alpha}\left(\nabla^{s} f\right)<\infty \text { for all } s \in \mathbb{N}_{0}^{n},|s|=k\right\}
$$

with

$$
\|f\|_{\mathrm{C}^{k, \alpha}(\bar{\Omega})}:=\|f\|_{\mathrm{C}^{k}(\bar{\Omega})}+\sum_{|s|=k} \operatorname{höl}_{\alpha}\left(\nabla^{s} f\right) .
$$

Functions in $\mathrm{C}^{0, \alpha}(\bar{\Omega})$ are called Hölder-continuous on $\bar{\Omega}$.

Functions in $\mathrm{C}^{0,1}(\bar{\Omega})$ are called Lipschitz-continuous on $\bar{\Omega}$.

\subsubsection{Definition (LebesGue-SpaCes $\mathrm{L}_{p}(\Omega)$ )}

Let $\Omega \subset \mathbb{R}^{n}$ open, $f: \Omega \rightarrow \mathbb{R}$ measurable and $p \in[1, \infty)$. We define

$$
\|f\|_{\mathrm{L}_{p}(\Omega)}:=\sqrt[p]{\int_{\Omega}|f|^{p} d x} \quad \text { and } \quad\|f\|_{\mathrm{L}_{\infty}(\Omega)}:=\operatorname{esssup}_{x \in \Omega}|f(x)| .
$$

Then the Lebesgue-space $\mathrm{L}_{p}(\Omega)$ is defined as

$$
\mathrm{L}_{p}(\Omega):=\left\{f: \Omega \rightarrow \mathbb{R} \mid f \text { measurable and }\|f\|_{\mathrm{L}_{p}(\Omega)}<\infty\right\} .
$$

REMARK 1.3.1 $\mathrm{L}_{2}(\Omega)$ with the scalar product $(f, g)_{\mathrm{L}_{2}(\Omega)}:=\int_{\Omega} f g$ is a Hilbert-space.

\subsubsection{Definition (SOBOlEv-SPACES $\mathrm{H}_{p}^{k}$ AND $\stackrel{\circ}{\mathrm{H}}{ }_{p}^{k}$ )}

Let $\Omega \subset \mathbb{R}^{n}$ open. Let $k \in \mathbb{N}_{0}$ and $p \in[1, \infty)$. We define

$$
\|f\|_{\mathrm{H}_{p}^{k}(\Omega)}:=\left(\sum_{|s| \leq k}\left\|\nabla^{s} f\right\|_{\mathrm{L}_{p}(\Omega)}^{p}\right)^{1 / p}
$$

and the Sobolev-spaces $\mathrm{H}_{p}^{k}(\Omega)$ and $\stackrel{\circ}{\mathrm{H}}_{p}^{k}(\Omega)$ as the closures

$$
\mathrm{H}_{p}^{k}(\Omega):=\overline{\left\{f \in \mathrm{C}^{\infty}(\Omega) \mid\|f\|_{\mathrm{H}_{p}^{k}(\Omega)}<\infty\right\}}
$$

and

$$
\stackrel{\circ}{\mathrm{H}}_{p}^{k}(\Omega):=\overline{\left\{f \in \mathrm{C}_{0}^{\infty}(\Omega) \mid\|f\|_{\mathrm{H}_{p}^{k}(\Omega)}<\infty\right\}}
$$

with respect to the $\mathrm{H}_{p}^{k}(\Omega)$ norm.

In the case of $p=2$ we set $\mathrm{H}^{k}(\Omega):=\mathrm{H}_{p}^{k}(\Omega)$ and $\stackrel{\circ}{\mathrm{H}}^{k}(\Omega):=\stackrel{\circ}{\mathrm{H}}{ }_{p}^{k}(\Omega)$. 
REMARK 1.3.2 $\mathrm{H}_{p}^{k}(\Omega)$ and $\stackrel{\circ}{\mathrm{H}}_{p}^{k}(\Omega)$ are Banach spaces, $\mathrm{H}_{2}^{k}(\Omega)$ and $\stackrel{\circ}{\mathrm{H}}_{2}^{k}(\Omega)$ with the scalar product $(f, g)_{\mathrm{H}_{2}^{k}(\Omega)}:=\sum_{|s| \leq k} \int_{\Omega} \nabla^{s} f \cdot \nabla^{s} g$ are Hilbert-spaces.

\subsubsection{DEFINITION (WEAK DERIVATIVE)}

Let $\Omega \subset \mathbb{R}^{n}$ open, $f: \Omega \rightarrow \mathbb{R}$ measurable and $s$ any multi-index. Then a locally integrable function $v: \Omega \rightarrow \mathbb{R}$ is called the $\mathrm{s}^{\text {th }}$ weak derivative of $f$ if it satisfies

$$
\int_{\Omega} v \varphi=(-1)^{|s|} \int_{\Omega} f \nabla^{s} \varphi \quad \text { for all } \varphi \in \mathrm{C}_{0}^{|s|}(\Omega)
$$

For $k \in \mathbb{N}_{0}$ the set of all $k$-times weakly differentiable functions is denoted by $\mathrm{W}^{k}(\Omega)$.

REMARK 1.3.3 We use the same notation for weak and for "ordinary" derivatives if it is unambiguous. So the $\mathrm{s}^{\text {th }}$ weak derivative of a function $f$ is denoted by $\nabla^{s} f$ and the like for the weak gradient, divergence and the directional derivatives.

Using Definition 1.3.9 it is possible to get an alternative characterisation of the Sobolev space $\mathrm{H}_{p}^{k}$ :

\subsubsection{Definition (The Space $\mathrm{W}_{p}^{k}$ )}

Let $\Omega \subset \mathbb{R}^{n}$ open. Let $k \in \mathbb{N}_{0}$ and $p \in[1, \infty)$. We define

$$
\mathrm{W}_{p}^{k}(\Omega):=\left\{f \in \mathrm{W}^{k}(\Omega) \mid \nabla^{s} f \in \mathrm{L}_{p}(\Omega) \text { for all }|s| \leq k\right\}
$$

\subsubsection{THEOREM}

Let $\Omega \subset \mathbb{R}^{n}$ open. Let $k \in \mathbb{N}_{0}$ and $p \in[1, \infty)$. Then it holds:

$$
\left(\mathrm{W}_{p}^{k}(\Omega),\|\cdot\|_{\mathrm{H}_{p}^{k}(\Omega)}\right)=\mathrm{H}_{p}^{k}(\Omega) .
$$

Proof: For example [Gilbarg and Trudinger 2001, Section 7.9] or [Evans 1998, Section 5.3]

\subsubsection{Differential geometry}

Analysis on manifolds is treated in detail - for example - in [AUBIN 1982], [CARmo 1992], [CARMo 1993] and relevant aspects can also be found in [WLOKA 1982] and [Gilbarg and Trudinger 2001]. However we do not need the notion of differentiability and curvature for the most general case, but only for the case of 2 dimensional isometric immersions into $\mathbb{R}^{3}$. Therefore we give the most basic definitions for the general case, but the covariant derivative and the curvature will only be defined for the case of $n$-dimensional isometric immersions into $\mathbb{R}^{n+1}$.

Differential manifolds For the most part the following definitions are cited from [AUBIN 1982] and also in some cases from [GALLOT et al. 1993]. 


\subsubsection{DEFINITION (MANIFOLD, CHART, PARAMETRISATION, ATLAS)}

1. A Hausdorff topological space $\Gamma$ is called a topological manifold of dimension $n$ if each point of $\Gamma$ has a neighbourhood homeomorphic to $\mathbb{R}^{n}$.

2. A local chart on $\Gamma$ is a pair $(\Omega, \phi)$ with $\Omega \subset \Gamma$ open and $\phi$ a homeomorphism of $\Omega$ onto an open set of $\mathbb{R}^{n}$.

3. The mapping $\phi^{-1}: \phi(\Omega) \rightarrow \Omega$ is called a parametrisation or coordinate system of $\Omega$.

4. A set $\left(\Omega_{i}, \phi_{i}\right)_{i \in I}$ of local charts of $\Gamma$ such that $\Gamma=\bigcup_{i \in I} \Omega_{i}$ is called an atlas of $\Gamma$.

\subsubsection{Definition ( $\mathrm{C}^{k}$-MANifold, orientable MANifold)}

An atlas $\left(\Omega_{i}, \phi_{i}\right)_{i \in I}$ of $\Gamma$ is called of class $\mathrm{C}^{k}$ if all changes of coordinate

$$
\phi_{i} \circ \phi_{j}^{-1}: \phi_{j}\left(\Omega_{i} \cap \Omega_{j}\right) \rightarrow \mathbb{R}^{n}
$$

are of class $\mathrm{C}^{k}$. Two atlases of class $\mathrm{C}^{k}$ are called equivalent if their union is an atlas of class $\mathrm{C}^{k}$. A differentiable manifold of class $\mathrm{C}^{k}$ is a manifold together with an equivalence class of $\mathrm{C}^{k}$ atlases. A differentiable manifold is called orientable if there exists an atlas so that the Jacobi-determinants for all changes of coordinate are positive.

Analogous definitions are valid for the differentiability classes $\mathrm{C}^{\infty}$ and $\mathrm{C}^{k, \alpha}$.

\subsubsection{DeFinition (DIFFERENTIABLE MAPPING, IMMERSiOn, EMBEDDinG)}

A mapping $f: \Gamma \rightarrow \Xi$ between two differentiable manifolds $\Gamma$ of dimension $n$ and $\Xi$ of dimension $m$, both of class $\mathrm{C}^{k}$, is called differentiable of class $\mathrm{C}^{r}$ in $P \in \Gamma, r \leq k$, if the mapping $\phi_{Q} \circ f \circ \phi_{P}^{-1}$ is differentiable of class $\mathrm{C}^{r}$ at $\phi_{P}(P)$ for local charts $\left(\Omega_{P}, \phi_{P}\right)$ around $P \in \Gamma$ and $\left(\Omega_{Q}, \phi_{Q}\right)$ around $Q:=f(P) \in \Xi$. The rank of $f$ in $P$ is defined as the rank of $\phi_{Q} \circ \phi_{P}^{-1}$ in $\phi_{P}(P)$.

A differentiable mapping $f$ is called immersion if its rank in each point of $\Gamma$ is equal to $n$. An immersion $f$ is called embedding if it is a homeomorphism of $\Gamma$ onto $f(\Gamma)$ where $f(\Gamma)$ has the topology induced by $\Xi$.

\subsubsection{Definition (TANGent Vector)}

$A$ tangent vector at $P \in \Gamma$ is a map $X: f \rightarrow X(f) \in \mathbb{R}$, defined on the set of real-valued functions differentiable in a neighbourhood of $P$, which satisfies

$$
\begin{aligned}
& \text { 1. } X(\lambda f+\mu g)=\lambda X(f)+\mu X(g) \quad \forall \lambda \mu \in \mathbb{R}, \\
& \text { 2. } X(f g)=f(P) X(g)+g(P) X(f)
\end{aligned}
$$

The tangent space $T_{P} \Gamma$ of $\Gamma$ at $P$ is the set of all tangent vectors at $P \in \Gamma$.

REMARK 1.3.4 It follows from 1. and 2. that $X(f)=0$ for all tangent vectors $X$ at $P \in \Gamma$ and all differentiable functions $f$ with $\nabla\left(f \circ \phi^{-1}\right)(\phi(P))=0$ for a local chart $(\Omega, \phi)$ around $P$. 
REMARK 1.3.5 The tangent space $T_{P} \Gamma$ of $\Gamma$ at $p$ has a natural vector space structure (as has any set of real-valued functions).

If $\Phi: \Omega \subset \mathbb{R}^{n} \rightarrow \Gamma$ is a coordinate system of a neighbourhood of $P$ then the tangent vectors

$$
\left(\frac{\partial}{\partial x_{i}}\right)_{P} \text { defined by }\left(\frac{\partial}{\partial x_{i}}\right)_{P}(f):=\left(\frac{\partial\left(f \circ \Phi^{-1}\right)}{\partial x_{i}}\right)_{\Phi^{-1}(P)}
$$

form a basis of $T_{P} \Gamma$.

\subsubsection{Definition (Tangent Space, Cotangent Space, Tensor Bundle)}

1. The tangent space $T \Gamma$ of $\Gamma$ is defined as the fiber bundle $T \Gamma:=\bigcup_{P \in \Gamma} T_{P} \Gamma$.

2. The cotangent space $T^{*} \Gamma$ of $\Gamma$ is defined as the fiber bundle $T^{*} \Gamma:=\bigcup_{P \in \Gamma} T_{P}^{*} \Gamma$, where $T_{P}^{*} \Gamma$ denotes the dual of $T_{P} \Gamma$.

3. The tensor bundle $T_{s}^{r} \Gamma$ of type $(r, s)$ of $\Gamma$ is defined as the bundle of tensor products $\bigcup_{P \in \Gamma} \stackrel{r}{\otimes} T_{P} \Gamma \stackrel{s}{\otimes} T_{P}^{*} \Gamma$.

1.3.17 Definition (Differential Section, Vector Field, Tensor Field)

1. A section of the fiber bundle $(E, \pi, \Gamma)$ is a differentiable map $\xi: \Gamma \rightarrow E$, such that $\pi \circ \xi(P)=P$ for all $P \in \Gamma$.

2. A differentiable vector field on $\Gamma$ is a section of $T \Gamma$, carrying its natural fiber bundle structure: In this case $\pi$ is the mapping $\pi: T \Gamma \rightarrow \Gamma: X \in T_{P} \Gamma \mapsto P$.

3. $A$ differentiable tensor field of type $(r, s)$ on $\Gamma$ is a section of $T_{s}^{r} \Gamma$.

\subsubsection{Definition (Metric, First Fundamental Form)}

$A$ Riemannian metric on $\Gamma$ is a differentiable tensor field $g$ of type $(0,2)$ on $\Gamma$ such that $g(P)$ is a scalar-product on $T_{P} \Gamma \times T_{P} \Gamma$.

$g$ is also called first fundamental form of the Riemannian manifold $\Gamma$.

Analysis on Hyper-Surfaces In the following $\Gamma \subset \mathbb{R}^{n+1}$ is an $n$-dimensional $\mathrm{C}^{2}$ immersion into $\mathbb{R}^{n+1}$. We identify the tangent space $T_{P} \Gamma$ at a point $P \in \Gamma$ with the affine hyper-plane of $\mathbb{R}^{n+1}$ which is tangential to $\Gamma$ at $P$.

$\Gamma$ has the Riemannian metric induced by the Euclidean metric of $\mathbb{R}^{n+1}$ so that $\Gamma \hookrightarrow \mathbb{R}^{n+1}$ by construction is an isometric immersion.

1.3.19 Definition (Tangential Derivatives, Laplace-Beltrami Operator) Let $\Gamma \subset \mathbb{R}^{n+1}$ be an isometric $\mathrm{C}^{2}$-immersion of dimension $n$. We denote its (local) outer unit normal field by $\nu_{\Gamma}=\left(\nu_{1}, \ldots, \nu_{n+1}\right): \Gamma \rightarrow \mathbb{R}^{n+1}$.

1. For a function $f \in \mathrm{C}^{1}(\Gamma)$ the tangential gradient $\nabla_{\Gamma} f$ is defined as

$$
\nabla_{\Gamma} f:=\nabla f-\left(\nabla f \cdot \nu_{\Gamma}\right) \nu_{\Gamma} .
$$

The tangential derivative $\delta_{i}^{\Gamma} f$ of $f$ in direction $i$ is defined as the $i$-th component of $\nabla_{\Gamma} \overline{f(1 \leq i \leq n+1) \text { : }}$

$$
\nabla_{\Gamma} f=\left(\delta_{1}^{\Gamma} f, \ldots, \delta_{n+1}^{\Gamma} f\right) .
$$


2. For a vector field $w=\left(w_{1}, \ldots, w_{n+1}\right) \in\left(\mathrm{C}^{1}(\Gamma)\right)^{n+1}$ the tangential divergence $\nabla_{\Gamma} \cdot w$ is defined as

$$
\nabla_{\Gamma} \cdot w:=\nabla \cdot w-\left(\nabla w_{i} \cdot \nu_{\Gamma}\right) \nu_{i}
$$

3. For a function $g \in \mathrm{C}^{2}(\Gamma)$ the Laplace-Beltrami operator $\Delta_{\Gamma} g$ is defined as

$$
\Delta_{\Gamma} g:=\nabla_{\Gamma} \cdot \nabla_{\Gamma} g
$$

REMARK 1.3.6 This definition makes sense only if $f$ and $w$ are extended to a neighbourhood of $\Gamma$, for example constant in normal direction. However, $\nabla_{\Gamma} f$ and $\nabla_{\Gamma} \cdot w$ do not depend on the extension, but only on the values of $f$ and $w$ on $\Gamma$.

Let $\left(e_{i} \mid 1 \leq i \leq n+1\right)$, denote the standard basis of $\mathbb{R}^{n+1}$. If $e_{i}, 1 \leq i \leq n+1$, is not orthogonal to $T_{P} \Gamma$ in $P \in \Gamma$, then a neighbourhood of $P$ can be parametrised as a graph over the hyperplane $E_{i}:=<e_{j} \mid 1 \leq j \leq n+1, j \neq i>_{\mathbb{R}}$. The components of $\nabla_{\Gamma} f$ are the covariant derivatives of $f$ in direction $e_{j}, j \neq i$. If $e_{i} \in\left(T_{P} \Gamma\right)^{\perp}$ then the corresponding component of $\nabla_{\Gamma} f$ is zero.

REMARK 1.3.7 In the following we will omit the upper index from the directional tangential derivatives whenever it is unambiguous, so $\delta_{i} f \equiv \delta_{i}^{\Gamma} f,(1 \leq i \leq n+1, f$ as in Definition 1.3.19 on the page before).

With $w$ and $g$ as in Definition 1.3.19 on the preceding page we get the following expressions for the tangential divergence and the Laplace-Beltrami operator:

$$
\nabla_{\Gamma} \cdot w=\delta_{i} w_{i}, \quad \Delta_{\Gamma} g=\delta_{i} \delta_{i} g
$$

\subsubsection{Definition (WEINGARTEN-MaP)}

Let $\Gamma \subset \mathbb{R}^{n+1}$ be an isometric $\mathrm{C}^{2}$-immersion of dimension $n$ with a (local) outer unit normal field $\nu_{\Gamma}=\left(\nu_{1}, \ldots, \nu_{n+1}\right): \Gamma \rightarrow \mathbb{R}^{n+1}$.

The Weingarten-map $I I=\left(I I_{1}, \ldots, I I_{n+1}\right): \Gamma \rightarrow \mathbb{R}^{(n+1) \times(n+1)}$ is defined as

$$
I I_{i}:=\nabla_{\Gamma} \nu_{i}, \quad 1 \leq i \leq n+1 .
$$

REMARK 1.3.8 Defined this way the Weingarten-map $I I$ is the usual second fundamental form (see for example [CARMO 1992]), but written in the coordinate frame of the embedding space $\mathbb{R}^{n+1}$, where the tangent space $T_{P} \Gamma$ at a point $P \in \Gamma$ is identified with the geometrical tangent hyper-plane (as sub-space of $\mathbb{R}^{n+1}$ ) of $\Gamma$ at $P$.

$T_{P} \Gamma<\mathbb{R}^{n+1}$ is an invariant sub-space of $I I(P) \in \operatorname{Hom}\left(\mathbb{R}^{n+1}, \mathbb{R}^{n+1}\right)$ and $I I(P)\left(\left(T_{p} \Gamma\right)^{\perp}\right)=\{0\}$, which implies that $\nu_{\Gamma}(P)$ is always an eigenvector of $I I(P)$ with eigenvalue 0 .

\subsubsection{Definition (Principal-, Mean- and Gaussian-Curvature)}

For $P \in \Gamma$ the eigenvalues $\kappa_{i}, 1 \leq i \leq n$ of the second fundamental form $\left.I I(P)\right|_{T_{p} \Gamma \times T_{P} \Gamma}$ are called principal curvatures of $\Gamma$ at $P$. The mean curvature $H_{\Gamma}$, the curvature vector $Y_{\Gamma}$ and the Gauss curvature $K_{\Gamma}$ at $P$ are defined as

$$
H_{\Gamma}:=\frac{1}{n} \sum_{i=1}^{n} \kappa_{i}, \quad Y_{\Gamma}:=n H_{\Gamma} \nu_{\Gamma}, \quad K_{\Gamma}:=\prod_{i=1}^{n} \kappa_{i} .
$$


REMARK 1.3.9 In the special case $n=2$ the mean and Gaussian curvature can be computed from $I I$ (instead of the restriction $\left.I I\right|_{T \Gamma \times T \Gamma}$ ) as follows:

$$
H_{\Gamma}=\frac{1}{2} \operatorname{trace} I I, \quad K_{\Gamma}=\operatorname{det}\left(I I+I_{3}\right)-2 H_{\Gamma}-1 .
$$

\subsubsection{Lemma (A Formula for the Mean Curvature Vector)}

Let $\Gamma \subset \mathbb{R}^{n+1}$ be an isometric $\mathrm{C}^{2}$-immersion of dimension $n$. Let $\operatorname{id}_{\Gamma}: \Gamma \rightarrow \Gamma: P \mapsto P$ be the identity mapping on $\Gamma$. Then the following holds

$$
-\Delta_{\Gamma} \operatorname{id}_{\Gamma}=Y_{\Gamma}
$$

Proof: Under the premises of Lemma 1.3 .22 let $\nu_{\Gamma}=\left(\nu_{1}, \ldots, \nu_{n+1}\right)$ be the outer unit normal field of $\Gamma$. We then have for a point $X=\left(X_{1}, \ldots, X_{n+1}\right) \in \Gamma$ and $1 \leq i \leq n+1$ :

$$
\begin{aligned}
\Delta_{\Gamma} X_{i}=\delta_{j} \delta_{j} X_{i} & =\delta_{j}\left(\nabla_{j} X_{i}-\left\langle\nabla X_{i}, \nu_{\Gamma}\right\rangle \nu_{j}\right) \\
& =\delta_{j}\left(\delta_{i j}-\nu_{i} \nu_{j}\right)=-\delta_{j}\left(\nu_{i} \nu_{j}\right) \\
& =-\underbrace{\nu_{j} \delta_{j} \nu_{i}}_{=0}-\nu_{i} \delta_{j} \nu_{j} \\
& =-\nu_{i} \delta_{j} \nu_{j} \\
& \stackrel{(1.5)}{=}-\operatorname{trace}(I I(X)) \nu_{i}=-n H_{\Gamma} \nu_{i} .
\end{aligned}
$$

\subsubsection{LEMma (INTEGRATION BY PARTS)}

For an isometric $\mathrm{C}^{2}$-immersion $\Gamma \subset \mathbb{R}^{n+1}$ without boundary and $f \in \mathrm{C}^{1}(\Gamma)$ the formula for integration by parts is

$$
\int_{\Gamma} \nabla_{\Gamma} f d o=-\int_{\Gamma} f Y_{\Gamma} d o
$$

Proof: A detailed proof is given in [Gilbarg and Trudinger 2001], chapter 16.

\subsubsection{COROLLARY}

From Lemma 1.3.23 it follows immediately for $f, g \in \mathrm{C}^{1}(\Gamma)$ :

$$
\int_{\Gamma} f \Delta_{\Gamma} g d o=\int_{\Gamma} \nabla_{\Gamma} f \cdot \nabla_{\Gamma} g d o
$$

Proof: Under the assumptions of Corollary 1.3.24 we have by product-rule:

$$
\int_{\Gamma} \nabla_{\Gamma} \cdot\left(f \nabla_{\Gamma} g\right) d o=\int_{\Gamma} \nabla_{\Gamma} f \cdot \nabla_{\Gamma} g d o+\int_{\Gamma} f \nabla_{\Gamma} \cdot \nabla_{\Gamma} g d o .
$$

On the other hand it follows from Lemma 1.3.23

$$
\int_{\Gamma} \nabla_{\Gamma} \cdot\left(f \nabla_{\Gamma} g\right) d o=-\int_{\Gamma} f \underbrace{\left\langle\nabla_{\Gamma} g, Y_{\Gamma}\right\rangle}_{=0} d o .
$$


Wloka defines in [WLOKA 1982, pp. 92] Sobolev-spaces $\mathrm{H}^{s}, s \in \mathbb{R}$, on Hölder-manifolds:

1.3.25 Definition (Sobolev-SPaCes $\mathrm{H}^{s}(\Gamma), \mathrm{H}^{-s}(\Gamma)$ )

Let $\Gamma$ be a compact Riemannian $\mathrm{C}^{k, \alpha}$-manifold of dimension $n, k \in \mathbb{N}_{0}, \alpha \in[0,1]$ with $\alpha=1$ in case $k=0$. Let $\left(\Omega_{l}, \Phi_{l}\right)_{l \in L}$ be a finite $\mathrm{C}^{k, \alpha}$-atlas of $\Gamma$ and $\phi_{l}$ a subordinate partition of unity (i.e. $\operatorname{supp}\left(\phi_{l}\right) \subset \Omega_{l}$ ). Let $s \leq k+\alpha$, if $\alpha \in\{0,1\}$ and $s<k+\alpha$ otherwise.

By definition $v: \Gamma \rightarrow \mathbb{R}$ belongs to $\mathrm{H}^{s}(\Gamma)$ if all functions

$$
\left(v \cdot \phi_{l}\right) \circ \Phi_{l}^{-1}: \Phi_{l}\left(\Omega_{l}\right) \rightarrow \mathbb{R}, l \in L,
$$

belong to $\stackrel{\circ}{\mathrm{H}}^{s}\left(\Phi_{l}\left(\Omega_{l}\right)\right)$. Together with the scalar-product

$$
\langle v, w\rangle_{s}:=\sum_{l}\left\langle\left(v \cdot \phi_{l}\right) \circ \Phi_{l}^{-1},\left(w \cdot \phi_{l}\right) \circ \Phi_{l}^{-1}\right\rangle_{\mathrm{H}^{s}\left(\mathbb{R}^{n}\right)}
$$

$\mathrm{H}^{s}(\Gamma)$ is a Hilbert-space.

For $s>0$ we denote by $\mathrm{H}^{-s}(\Gamma)$ the dual space of $\mathrm{H}^{s}(\Gamma)$.

REMARK 1.3.10 The above definition implicitly defines the notion of weak derivatives for a $\mathrm{C}^{k, \alpha}$-manifold, using the same construction as in the definition of differential mappings on manifolds, Definition 1.3.14 on page 10 .

[Wloka 1982, pp. 92] shows that the Sobolev-spaces $\mathrm{H}^{s}(\Gamma)$ - given the prerequisites of Definition 1.3.25 - are well-defined and in particular that $\langle\cdot, \cdot\rangle_{s}$ only depends up to norm-equivalence on the special choice of the atlas and the local chart.

Definition 1.3.25 covers especially the case of the discrete surfaces of class $\mathrm{C}^{0,1}$ constructed by the finite element approach of Section 2.4.1 on page 42 below.

\subsubsection{Some aspects from bifurcation theory}

The following section gives in brief some notations needed to motivate the approach sketched in Chapter 2. We cite from [CHOW and HaLE 1996].

For the remainder of this section we assume that $X$ and $Z$ are Banach-spaces and $\Lambda$ is an open set in a Banach space. $M: \Lambda \times X \rightarrow Z$ is continuously Frechet differentiable. $D_{x} M(\lambda, x)$ denotes the Frechet-derivative of $M$ with respect to its second argument evaluated at $(\lambda, x) . \Lambda$ is called the parameter set. We are interested in solutions of the equation

$$
M(\lambda, x) \stackrel{!}{=} 0
$$

for $\lambda \in \Lambda, x \in X$. Let $\mathcal{S} \subset \Lambda \times X$ denote the set of solutions of (1.11) and for any $\lambda \in \Lambda$ define

$$
\mathcal{S}_{\lambda}:=\{x \in \mathcal{S} \mid(\lambda, x) \in \mathcal{S}\}
$$




\subsubsection{Definition (Bifurcation Point)}

For any open set $U \subset X$ we define the equivalence relation $\sim_{U}$ on the set $\left\{\mathcal{S}_{\lambda} \mid \lambda \in \Lambda\right\}$ by setting $\mathcal{S}_{\lambda} \sim_{U} \mathcal{S}_{\mu}: \Longleftrightarrow \mathcal{S}_{\lambda} \cap U$ is homeomorphic to $\mathcal{S}_{\mu} \cap U$. A point $\lambda_{c} \in \Lambda$ is called a bifurcation point for $\mathcal{S}$ if, for any neighbourhood $V \subset \Lambda$ of $\lambda_{c}$, there is an $x_{c} \in \mathcal{S}_{\lambda_{c}}$ and a neighbourhood $U \subset X$ of $x_{c}$ such that there are $\lambda_{1}, \lambda_{2} \in V$ with $\mathcal{S}_{\lambda_{1}} \chi_{U} \mathcal{S}_{\lambda_{2}}$.

REMARK 1.3.11 Particularly, $\lambda_{c} \in \Lambda$ is called a bifurcation point if there is an $x_{c} \in \mathcal{S}_{\lambda_{c}}$ and a neighbourhood $V_{c} \times U_{c} \subset \Lambda \times X$ of $\left(\lambda_{c}, x_{c}\right)$ such that for any $\lambda \in V_{c} \backslash\left\{\lambda_{c}\right\}$ there is more than one solution, that is, $\left|\mathcal{S}_{\lambda}\right|>1$. We note that Definition 1.3.26 covers more general types of bifurcations.

We state now a version of the implicit function theorem on Banach spaces.

\subsubsection{Theorem (Implicit Function TheOREM)}

Suppose $X, Y, Z$ are Banach spaces, $U \subset X, V \subset Y$ are open sets, $F: U \times V \rightarrow Z$ is continuously (Frechet-)differentiable, $\left(x_{0}, y_{0}\right) \in U \times V, F\left(x_{0}, y_{0}\right)=0$ and its Frechetderivative $D_{x} F\left(x_{0}, y_{0}\right)$ with respect to $x$ has a bounded inverse.

Then there is a neighbourhood $U_{1} \times V_{1} \subset U \times V$ of $\left(x_{0}, y_{0}\right)$ and a function $f: V_{1} \rightarrow U_{1}$, $f\left(y_{0}\right)=x_{0}$ such that $F(x, y)=0$ for $(x, y) \in U_{1} \times V_{1}$ if and only if $x=f(y)$.

If $F \in \mathrm{C}^{k}(U \times V, Z), k \geq 1$ or analytic in a neighbourhood of $\left(x_{0}, y_{0}\right)$, then $f \in \mathrm{C}^{k}\left(V_{1}, X\right)$ or $f$ is analytic in a neighbourhood of $y_{0}$.

Proof: [CHOw and Hale 1996, Section 2.2]

This means that $D_{x} F\left(x_{0}, y_{0}\right)$ has no bounded inverse at a bifurcation point.

The Liapunov-Schmidt method reduces the bifurcation problem for a certain class of problems to solving a finite set of equations in a finite number of unknowns. We will omit a description of this method here and only cite [CHOW and HALE 1996] with the following result which is based on the method of Liapunov-Schmidt:

1.3.28 Theorem (Bifurcation from a Simple Eigenvalue)

Let $X$ and $Z$ be real Banach-spaces, $\Lambda \subset \mathbb{R}$ open and $M: \Lambda \times X \rightarrow Z$ defined by

$$
\begin{aligned}
& M(\lambda, x)=B x-\lambda A x+N(\lambda, x), \\
& N(\lambda, 0)=0, \quad D_{x} N(\lambda, 0)=0,
\end{aligned}
$$

with $B, A \in L(X, Z)$ and $N \in \mathrm{C}^{1}(\Lambda \times X, Z)$ with continuous mixed second Frechetderivative $D_{\lambda x} N$.

If $\lambda_{0}$ is a simple eigenvalue of the pair $(B, A)$ with eigenvector $y_{0}$, then there is a $\delta>0$ and $\mathrm{C}^{1}$-functions

$$
\begin{aligned}
& \lambda^{*}(u)=\lambda_{0}+O(\|u\|), \\
& x^{*}(u)=u y_{0}+O\left(\|u\|^{2}\right)
\end{aligned}
$$

for $|u|<\delta, u \in \mathbb{R}$, such that $M\left(\lambda^{*}(u), x^{*}(u)\right)=0$. All zeroes of $M$ near $\left(\lambda_{0}, 0\right)$ are either $(\lambda, 0)$ or given by $\left(\lambda^{*}(u), x^{*}(u)\right)$. 
Proof: [CHOW and HALE 1996, remarks at the beginning of Section 5.5 and Theorem $5.3]$

REMARK 1.3.12 If $\lambda_{0}$ is an eigenvalue of the pair $(B, A)$ with eigenvector $y_{0}$, then $\left(B-\lambda_{0} A\right) y_{0}=0$. As $D_{x} M(\lambda, x)=B-\lambda_{0} A$, Theorem 1.3.28 on the preceding page states that under the given assumptions it is sufficient to look at the eigenvalues of $D_{x} M(\lambda, x)$ to determine a bifurcation point.

Moreover, the eigenvector $y_{0}$ approximates the solution-family branching off at $\left(\lambda_{0}, 0\right)$ in the sense stated more precisely in equation (1.12) above.

\subsection{Exact solutions}

Up to now two families of exact solutions to the Euler-Lagrange equations (1.3) are known. One was already discovered by A. Beer [BEER 1869] in the 19th century. It is a one-parameter family of drops having the topological type of a sphere. The other one was detected by R. Gulliver [Gulliver 1984] in 1984 and has the topology of a torus.

Both families consist of surfaces of revolution where the generating curve is given in the following integral form:

$$
\begin{gathered}
u(r)=\int_{r_{-}}^{r} \frac{v(\rho)}{\sqrt{1-v^{2}(\rho)}} d \rho, r_{-} \leq r \leq r_{+} \\
v(\rho):=\omega^{2} \rho^{3}+a \rho+b / \rho
\end{gathered}
$$

$b$ and $c$ are constants which depend on the angular velocity $\omega$. For drops of the topological type of the sphere one has $r_{-}=0, r_{+}$is the equatorial radius, $b=0$ and $a$ is the mean curvature of the drop at the axis of rotation.

For annular drops $r_{-}$and $r_{+}$denote the inner and outer radii of the torus.

Both families meet at a point where the inner radius of the annular shapes and the thickness at the axis of rotation of the simply connected drops both approach 0 (Figure 1.4).

Figure 1.4 Limit surfaces of annular and simply connected family

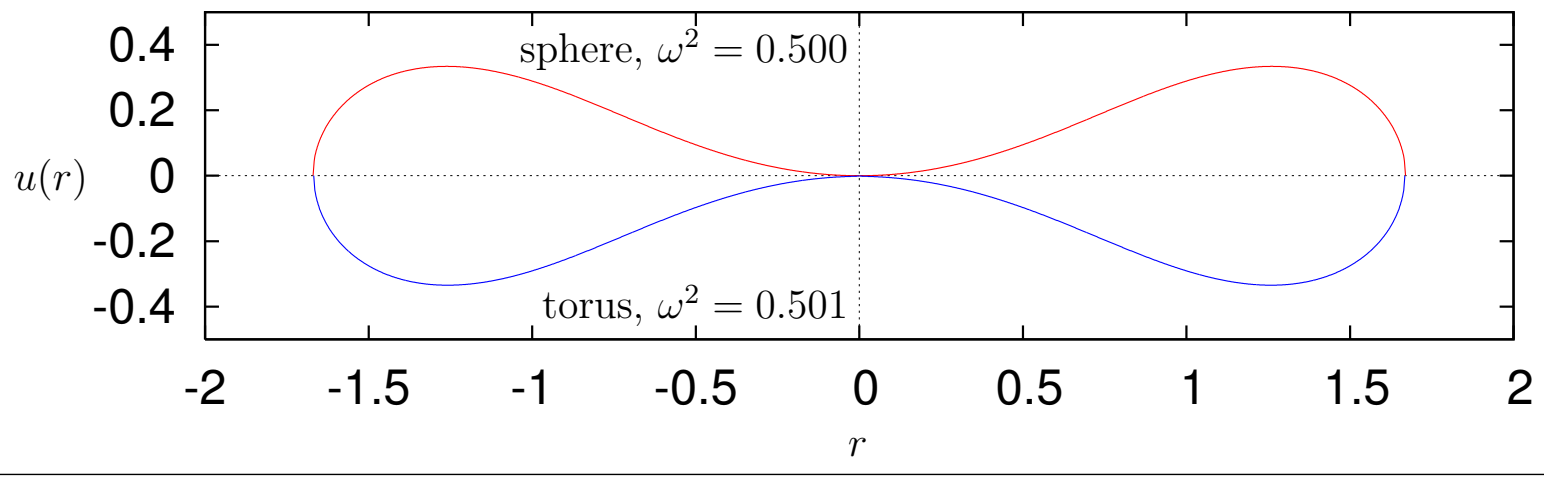


Figure $1.5 \omega-L$ phase diagram for exact annular and simply connected solutions.

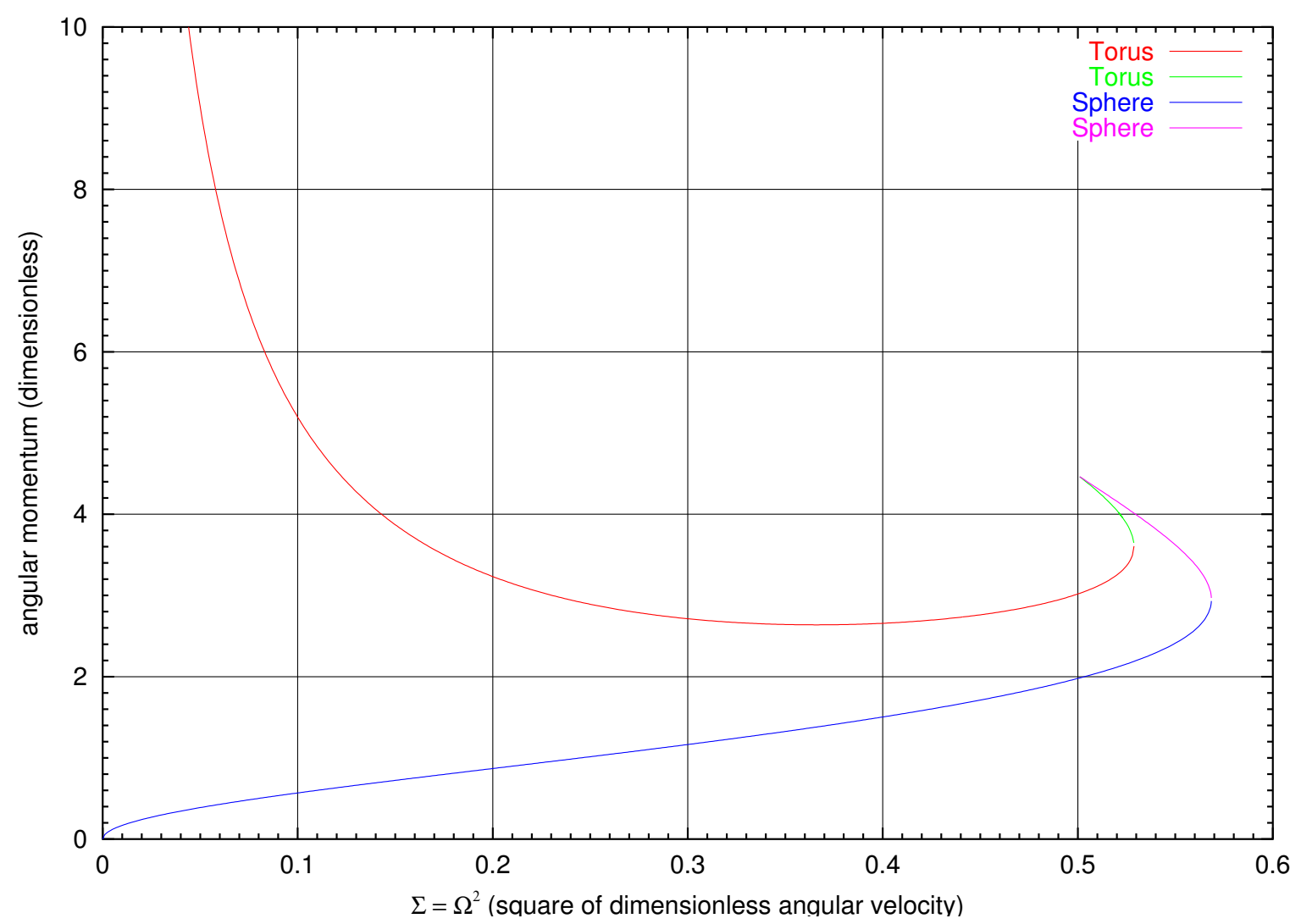

The value of $\omega$ at the "meeting point" in the chosen normalisation is exactly $\omega^{2}=1 / 2$ (see equation (1.15) below and Figure 1.5).

Obviously, between the meeting point and the turning point (maximal value of $\omega$ ) of each of the families there are two solutions for each value of $\omega$.

\subsubsection{Solutions of spheroidal type}

A derivation of the formulas for the coefficient $a$ in (1.13) on the preceding page can be found in [BEER 1869, pp. 158]. A short summary is also in the appendix of [CHANDRASEKHAR 1965], for example. We will just present the resulting expressions.

To get an explicit expression for $a$ in (1.13) one has to use the parameter $k$ defined as

$$
k:=\frac{8 \sigma}{\rho \hat{\omega}^{2} r_{1}^{3}}=\frac{1}{r_{1}^{3} \bar{\omega}^{2}} \equiv \frac{1}{r_{1}^{3} \omega^{2}}
$$

instead of the angular velocity $\omega$ (normalisation as chosen in Section 1.1). Here $r_{1}$ denotes the radius of the drop at is equator. 
Figure 1.6 Computation of the spheroidal drop shapes.

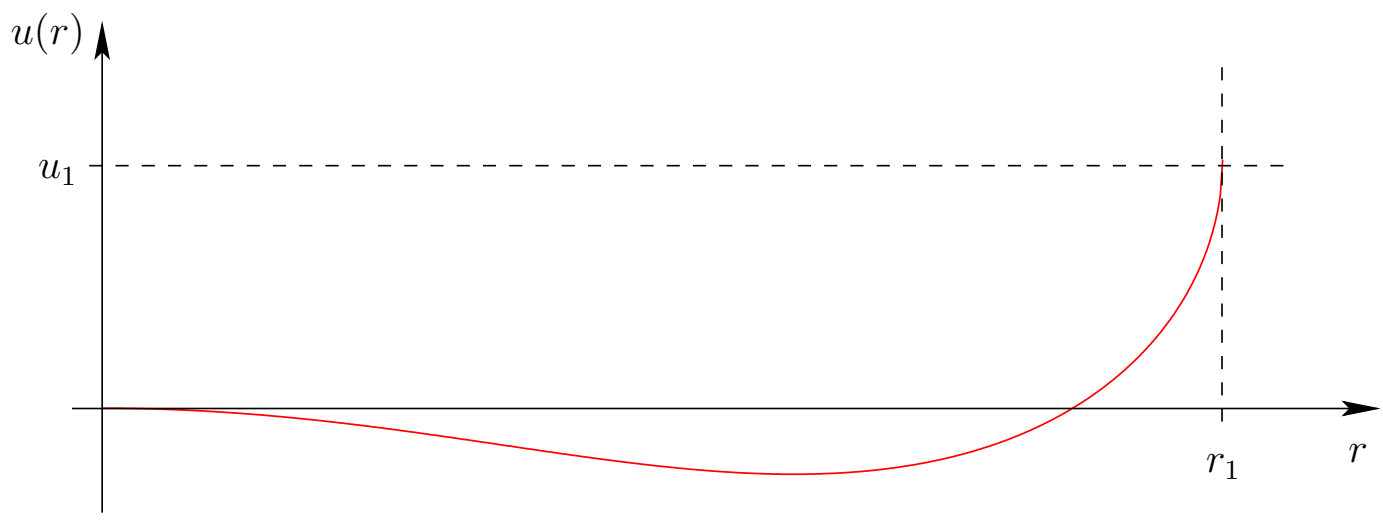

Using the elliptic integrals $F(c, \varphi)$ and $E(c, \varphi)$ of first and second kind in Legendre normal form,

$$
F\left(c, \varphi_{1}\right):=\int_{0}^{\varphi_{1}} \frac{d \varphi}{\sqrt{1-c^{2} \sin ^{2} \varphi}} \quad \text { and } \quad E\left(c, \varphi_{1}\right):=\int_{0}^{\varphi_{1}} \sqrt{1-c^{2} \sin ^{2} \varphi} d \varphi
$$

one first expresses the ratio $u_{1} / r_{1}$ of half of the drop's thickness at the axis of rotation to the equatorial radius $r_{1}$ (see Figure 1.6):

$$
\frac{u_{1}}{r_{1}}=\frac{u\left(r_{1}\right)}{r_{1}}=\left(\frac{k}{2 m}-\frac{m}{2}\right) F\left(c, \varphi_{1}\right)+m E\left(c, \varphi_{1}\right)-\sqrt{1-c^{2} \sin ^{2} \varphi_{1}},
$$

with

$$
\begin{aligned}
m & =\sqrt[4]{2 k+k^{2}} \\
c & =\frac{1}{m} \sqrt{\frac{m^{2}}{2}+\frac{1+2 k}{4}}=\sqrt{\frac{1}{2}+\frac{1+2 k}{4 m^{2}}}=\sqrt{\frac{1}{2}+\frac{1+2 k}{4 \sqrt{2 k+k^{2}}}} \\
\varphi_{1} & =2 \arctan \frac{1}{m}=2 \arctan \frac{1}{\sqrt[4]{2 k+k^{2}}} .
\end{aligned}
$$

Then one uses the volume constraint to compute the constants $\omega^{2}, r_{1}$ and $a$ as functions of $k$ :

$$
\omega^{2}=\frac{1}{2}\left(1-\frac{u_{1}}{r_{1}} \frac{k-1}{k}\right), \quad r_{1}=\sqrt[3]{\frac{1}{\omega^{2} k}}, \quad a=\frac{1}{r_{1}} \frac{k-1}{k}
$$

The generating curve $u(r)$ of the surface of revolution can then be computed from its integral representation (1.13) with $b=0, r_{-}=0$ and $r_{+}=r_{1}$. Clearly, for a drop of thickness 0 at $r=0$ one has $u_{1}=0$ and therefore $\omega^{2}=1 / 2$. Figure 1.7 on the next page gives an overview over the possible drop shapes for some values of $\omega$. 
$\overline{\text { Figure 1.7 Meridional sections through some spheroidal shapes at different values of }}$ $\omega$. Only the "right" half of the section is shown. The abscisse shows the distance to the axis of rotation. The thickness of the shapes at $r=0$ is a monotonically increasing function of the parameter $k$ defined in (1.14).

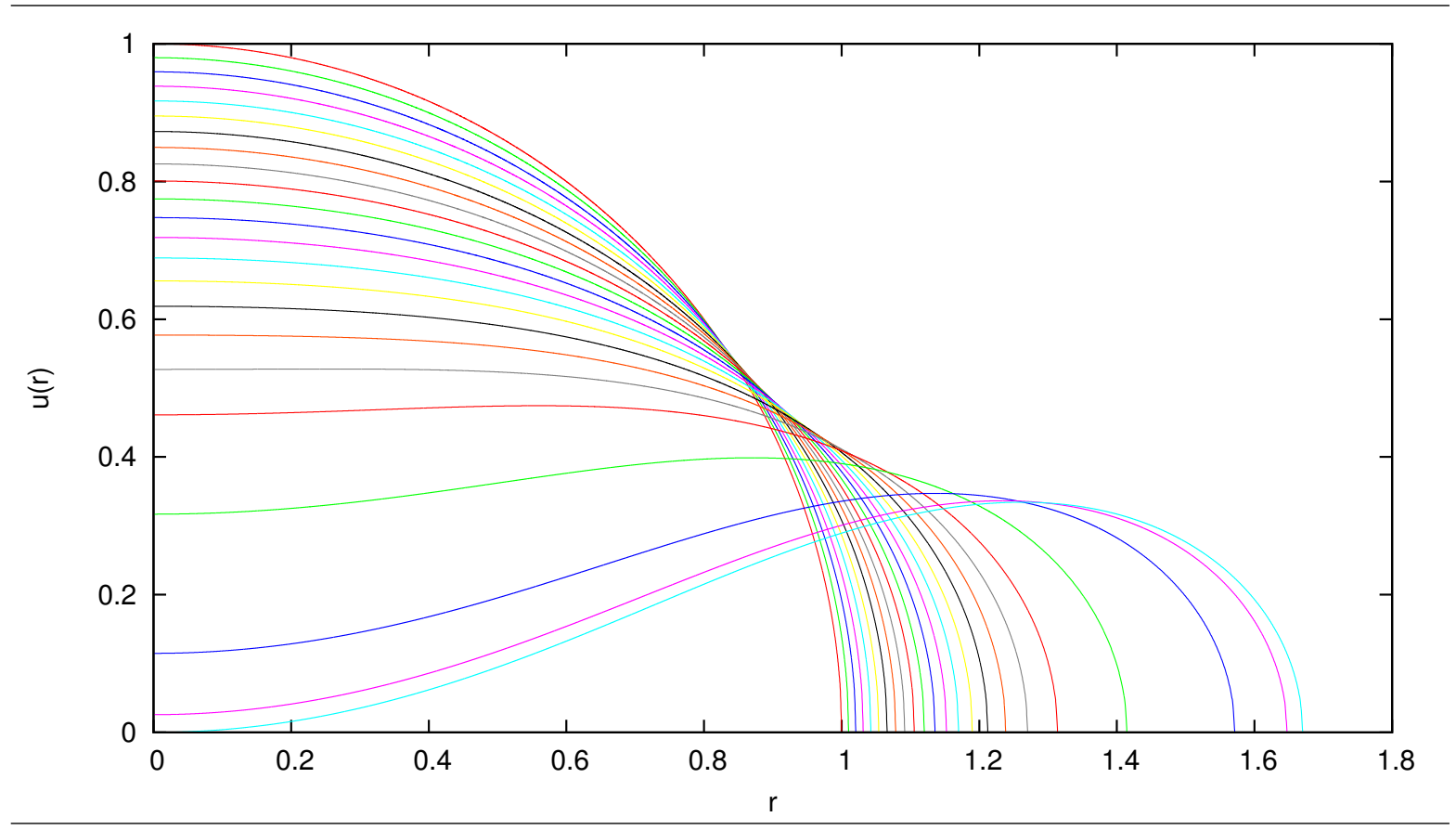

Figure 1.8 Meridional section through some annular shapes at different values of $\omega^{2}$. Only the "right" half of the section is shown. The abscisse shows the distance to the axis of rotation. One observes that the equatorial radius of the torus becomes very large for small $\omega$.

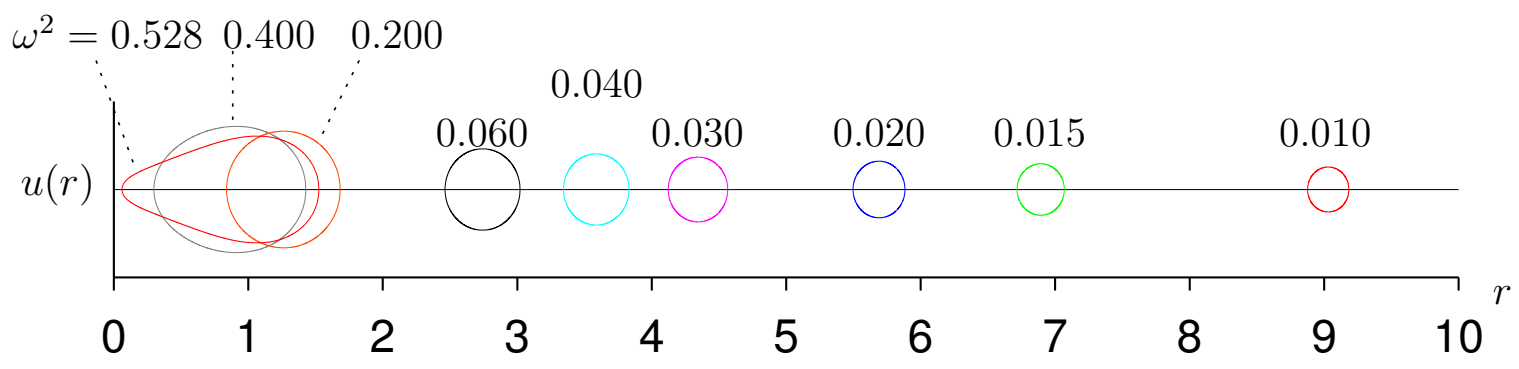

\subsubsection{Solutions of annular type}

Gulliver [GULLIVER 1984] proves the existence of a family of tori as well as the existence of the meeting point with the spheroidal family (Figure 1.5 on page 17). However, the constants $a, b, r_{-}$and $r_{+}$are not given in closed form but have to be computed 
numerically from the following equations ( $\omega^{2}$ given):

(1.16a) $\quad 1 \stackrel{!}{=} \omega^{2} r_{-}^{3}+a r_{-}+b \frac{1}{r_{-}} \stackrel{!}{=} \omega^{2} r_{+}^{3}+a r_{+}+b \frac{1}{r_{+}} \quad$ (smoothness at equator),

(1.16b) $\quad 0 \stackrel{!}{=} F(a, b):=\int_{r_{-}}^{r_{+}} \frac{\omega^{2} \rho^{3}+a \rho+b \frac{1}{\rho}}{\sqrt{1-\left(\omega^{2} \rho^{3}+a \rho+b \frac{1}{\rho}\right)^{2}}} d \rho \quad$ (surface must be closed)

(1.16c) $\frac{2 \pi}{3} \stackrel{!}{=} V(a, b):=\pi \int_{r_{-}}^{r_{+}} \rho^{2} \frac{\omega^{2} \rho^{3}+a \rho+b \frac{1}{\rho}}{\sqrt{1-\left(\omega^{2} \rho^{3}+a \rho+b \frac{1}{\rho}\right)^{2}}} d \rho \quad$ (volume constraint). 


\section{Chapter 2}

\section{The path tracking problem}

In this chapter we will first derive expressions for the first and second variation of the (normalised) energy $E$ of a drop which rotates rigidly at constant angular velocity $\omega$, held together by surface tension alone:

$$
E(\Gamma, C)=|\Gamma|-4 \omega^{2} \int_{\Omega} r^{2}-C(3|\Omega|-4 \pi) .
$$

We use the notation of Section 1.1 which means that $\Omega \subset \mathbb{R}^{3}$ is the interior of the drop, bounded by the surface $\Gamma:=\partial \Omega$. The distance from the axis of rotation is denoted by $r=\sqrt{x_{1}^{2}+x_{2}^{2}}$, where $\mathbb{R}^{3}=\left\{\left(x_{1}, x_{2}, x_{3}\right) \mid x_{1}, x_{2}, x_{3} \in \mathbb{R}\right\} . C \in \mathbb{R}$ is the Lagrangeparameter for the volume constraint.

"Solutions" are pairs $(\Gamma, C)$ which are critical points of $E$, where $\Gamma \subset \mathbb{R}^{3}$ is a compact closed immersion of dimension 2 and $C \in \mathbb{R}$.

Under the assumption that the prerequisites of Theorem 1.3.28 on page 15 hold we know that the solution set of $E$ is locally - and in the absence of bifurcation points - a oneparameter family, parametrised by the angular velocity $\omega$. Branch-points in the sense of Definition 1.3.26 on page 15 are just points where two or more one-parameter families come together.

Before arriving at a fully-discrete scheme for the computation of drop shapes we will first use the second variation of $E$ to construct a Newton iteration for a semi-discrete regime where each step of the Newton iteration is established by solving certain partial differential equations exactly. This is described in Section 2.3 below.

The fully-discrete scheme is introduced by means of a finite element method in Section 2.4 .

In this setup "path-tracking" means that we start with an exactly known solution $\left(\Gamma_{0}, C_{0}\right)$ at an angular velocity $\omega_{0}$ and use this solution as initial value for the Newton scheme to construct a new solution $\left(\Gamma_{1}, C_{1}\right)$ at a different angular velocity $\omega_{1}$. This is iterated to compute a discrete subset $\left\{\left(\Gamma_{i}, C_{i}\right) \mid i \in \mathbb{N}\right\}$ of the solution set of $E$, always using the previous solution $\left(\Gamma_{i-1}, C_{i-1}\right)$ as initial value for the Newton iteration which constructs $\left(\Gamma_{i}, C_{i}\right)$. 
To get a more thorough picture of the solution set the path-tracking algorithm checks for bifurcation points between $\left(\Gamma_{i-1}, C_{i-1}\right)$ and $\left(\Gamma_{i}, C_{i}\right)$. If there is a bifurcation point a new instance of the path-tracking algorithm is started to track down the new branches originating from there.

We start by deriving the full and linearised Euler-Lagrange equations for the critical points of $E$.

\subsection{First and second variation of the energy func- tional}

In the following $\Gamma \subset \mathbb{R}^{3}$ is assumed to be an immersed compact surface of class $\mathrm{C}^{k, \alpha}$ with $k \in \mathbb{N}, k \geq 3, \alpha \in(0,1)$. We will be more specific on the value of $k$ and $\alpha$ when we are discussing the linearised Euler-Lagrange equation below.

We will give expressions for the first and second variation of

$$
\begin{aligned}
E(\Gamma, C) & =|\Gamma|-4 \omega^{2} \int_{\Omega} r^{2} d x-C(3|\Omega|-4 \pi) \\
& =\underbrace{\int_{\Gamma} d o}_{=: E_{\text {surf }}(\Gamma)} \underbrace{-4 \omega^{2} \int_{\Omega} r^{2} d x}_{=: E_{\mathrm{rot}}(\Gamma)} \underbrace{-C\left(\int_{\Gamma} \mathrm{id}_{\Gamma} \cdot \nu_{\Gamma} d o-4 \pi\right)}_{=: E_{\mathrm{vol}}(\Gamma, C)} .
\end{aligned}
$$

and the corresponding Euler-Lagrange equations. The second variation $\delta^{2} E$ will be derived for perpendicular perturbations only. $\delta^{2} E$ will be given in scalar terms of the signed length of the perturbations as well as in terms of the perturbations themselves.

The following is of course well known; the variation of the surface energy is treated in great detail in [NITSCHE 1975], for example.

\subsubsection{The first variation of $E(\Gamma, C)$}

Let $\varphi \in\left(\mathrm{C}^{k, \alpha}(\Gamma)\right)^{3}$. For $\varepsilon>0$ define the variation $\Gamma_{\varphi}(\varepsilon)$ of $\Gamma$ with respect to $\varphi$ as

$$
\Gamma_{\varphi}(\varepsilon):=\{x+\varepsilon \varphi(x) \mid x \in \Gamma\}
$$

We note that $\Gamma_{\varphi}(\varepsilon)$ is again of class $\mathrm{C}^{k, \alpha}$ and defines again an immersed compact surface provided $\varepsilon$ is small enough. This follows from the boundedness of the curvature of $\Gamma$.

Then, for $K \in \mathbb{R}$ the first variation $\delta E(\Gamma, C)[\varphi, K]$ of $E$ at $(\Gamma, C)$ in direction of $(\varphi, K)$ is

$$
\delta E(\Gamma, C)[\varphi, K]=\left.\frac{d}{d \varepsilon} E\left(\Gamma_{\varphi}(\varepsilon), C+\varepsilon K\right)\right|_{\varepsilon=0} .
$$


First variation $\delta E_{\text {surf }}$ of the surface energy

$$
\begin{aligned}
\delta E_{\text {surf }}(\Gamma) \varphi & =\left.\frac{d}{d \varepsilon} E_{\text {surf }}\left(\Gamma_{\varphi}(\varepsilon)\right)\right|_{\varepsilon=0}=\left.\frac{d}{d \varepsilon} \int_{\Gamma_{\varphi}(\varepsilon)} d o_{\varepsilon}\right|_{\varepsilon=0} \\
& =\int_{\Gamma} \nabla_{\Gamma} \operatorname{id}_{\Gamma}: \nabla_{\Gamma} \varphi d o \stackrel{(1.10)}{=}-\int_{\Gamma} \Delta_{\Gamma} \mathrm{id}_{\Gamma} \cdot \varphi d o .
\end{aligned}
$$

First variation $\delta \boldsymbol{E}_{\text {rot }}$ of the rotational energy Obviously, the first variation is just the surface integral over $\Gamma$ of the centrifugal energy-density $-4 \omega^{2} r^{2}$ multiplied by the distance of the perturbed surface $\Gamma_{\varphi}(\varepsilon)$ from the original surface $\Gamma$ :

$$
\delta E_{\mathrm{rot}}\left(\Gamma_{\varphi}(\varepsilon)\right) \varphi=\left.\frac{d}{d \varepsilon} E_{\mathrm{rot}}\left(\Gamma_{\varphi}(\varepsilon)\right)\right|_{\varepsilon=0}=-4 \omega^{2} \int_{\Gamma} r^{2} \varphi \cdot \nu_{\Gamma} d o
$$

This is clear from the additivity of the Riemann-integral (this is the classical setup, $\Gamma$ is of class $\left.\mathrm{C}^{k, \alpha}\right)$.

First variation $\boldsymbol{\delta} \boldsymbol{E}_{\mathrm{vol}}$ of the volume constraint From the same reasoning as is used in the case of $\delta E_{\text {rot }}\left(\Gamma_{\varphi}(\varepsilon)\right) \varphi$ above follows:

$$
\begin{aligned}
\delta E_{\mathrm{vol}}(\Gamma, C)[\varphi, K] & =\left.\frac{d}{d \varepsilon} E_{\mathrm{vol}}\left(\Gamma_{\varphi}(\varepsilon), C+\varepsilon K\right)\right|_{\varepsilon=0} \\
& =-C \int_{\Gamma} \varphi \cdot \nu_{\Gamma} d o-K\left(\int_{\Gamma} \mathrm{id}_{\Gamma} \cdot \nu_{\Gamma} d o-4 \pi\right) .
\end{aligned}
$$

Summarising the above formulas (2.2)-(2.4) we get the following expression for the first variation $\delta E(\Gamma, C)[\varphi, K]$ of the energy $E(\Gamma, C)$ in direction of $(\varphi, K)$ :

$$
\begin{aligned}
\delta E(\Gamma, C)[\varphi, K]= & \left.\frac{d}{d \varepsilon} E\left(\Gamma_{\varphi}(\varepsilon), C+\varepsilon K\right)\right|_{\varepsilon=0} \\
= & -\int_{\Gamma} \Delta_{\Gamma} \mathrm{id}_{\Gamma} \cdot \varphi d o-4 \omega^{2} \int_{\Gamma} r^{2} \varphi \cdot \nu_{\Gamma} d o .-C \int_{\Gamma} \varphi \cdot \nu_{\Gamma} d o \\
& -K\left(\int_{\Gamma} \mathrm{id}_{\Gamma} \cdot \nu_{\Gamma} d o-4 \pi\right) .
\end{aligned}
$$

By definition it holds for a stationary point $\delta E(\Gamma, C)[\varphi, K]=0$ for all $(\varphi, K) \in \mathrm{C}^{\alpha, k}(\Gamma) \times \mathbb{R}$. So it follows from the fundamental Lemma of the calculus of variations that a stationary point of $E$ satisfies the Euler-Lagrange equations:

$$
-\Delta_{\Gamma} \mathrm{id}_{\Gamma}=\left(4 \omega^{2} r^{2}+C\right) \nu_{\Gamma}, \quad \int_{\Gamma} \operatorname{id}_{\Gamma} \cdot \nu_{\Gamma} d o=4 \pi .
$$

This is exactly equation (1.3) on page 3 from the introductory section Section 1.1.

REMARK 2.1.1 (2.6) is a partial differential equation defined on a surface which is itself the major unknown in (2.6). This seems to be a "chicken and egg" problem. But, this, however is not so. (2.6) has to be understood in terms of either local charts, or by interpreting $\mathrm{id}_{\Gamma}$ as a function on another, explicitly known manifold close (in some sense) to a solution $\Gamma$ of (2.6).

In either case the above Euler-Lagrange equations are a system of coupled, highly nonlinear partial differential equations. 


\subsubsection{The second variation of $E(\Gamma, C)$}

The expressions for the second variation $\delta^{2} E$ of $E$ are far more complicated than equation (2.4). Particularly, it makes a major difference whether one allows arbitrary perturbations or only perturbations perpendicular to the surface $\Gamma$, and the interconnection between different kinds of perturbations is quite involved.

In the following we only consider orthogonal perturbations $\varphi \in\left(\mathrm{C}^{k-1, \alpha}(\Gamma)\right)^{3}$, where "orthogonal" means $\langle\varphi, \nu\rangle \varphi=\varphi$. For any $\varphi \in\left(\mathrm{C}^{k-1, \alpha}(\Gamma)\right)^{3}$ we define $\bar{\varphi}:=\langle\varphi, \nu\rangle$.

First we state some well-known identities which are needed in the derivation of the second variation.

\subsubsection{Lemma (Calculus on Surfaces)}

Let $\Gamma \subset \mathbb{R}^{3}$ be an immersed surface of class $\mathrm{C}^{k, \alpha}$ and $\psi \in\left(\mathrm{C}^{k-1, \alpha}(\Gamma)\right)^{3}$ an orthogonal variation with $k \geq 3, \alpha \in(0,1)$. Then the following holds:

Variation of the unit normal field

$$
\left.\frac{d}{d \varepsilon}\left(\nu_{\Gamma_{\psi}(\varepsilon)}\right)\right|_{\varepsilon=0}=-\left(\nabla_{\Gamma} \psi\right)^{t r} \nu_{\Gamma}
$$

\section{Variation of the curvature vector}

$$
\begin{aligned}
-\left.\frac{d}{d \varepsilon}\left(Y_{\Gamma_{\psi}(\varepsilon)}\right)\right|_{\varepsilon=0} & =\left.\frac{d}{d \varepsilon}\left(\Delta_{\Gamma_{\psi}(\varepsilon)} \operatorname{id}_{\Gamma_{\psi}(\varepsilon)}\right)\right|_{\varepsilon=0} \\
& =\nu_{\Gamma} \nu_{\Gamma} \cdot \Delta_{\Gamma} \psi+2 \nu_{\Gamma} \nabla_{\Gamma} \psi: \nabla_{\Gamma} \nu_{\Gamma}+\left(\nabla_{\Gamma} \psi\right)^{t r} \cdot Y_{\Gamma} .
\end{aligned}
$$

\section{Divergence of an orthogonal perturbation}

$$
\nabla_{\Gamma} \cdot \psi=\psi \cdot Y_{\Gamma}
$$

\section{Square of the Weingarten map}

$$
\nabla_{\Gamma} \nu_{\Gamma}: \nabla_{\Gamma} \nu_{\Gamma}=\kappa_{1}^{2}+\kappa_{2}^{2}=-\nu_{\Gamma} \cdot \Delta_{\Gamma} \nu_{\Gamma}
$$

where $\kappa_{1}$ and $\kappa_{2}$ denote the principal curvatures of $\Gamma$.

\section{Laplace-Beltrami operator of an orthogonal perturbation}

$$
\nu_{\Gamma} \cdot \Delta_{\Gamma} \psi=\Delta_{\Gamma} \bar{\psi}+\bar{\psi}\left(\nu_{\Gamma} \cdot \Delta_{\Gamma} \nu_{\Gamma}\right) \stackrel{(2.10)}{=} \Delta_{\Gamma} \bar{\psi}-\bar{\psi}\left(\nabla_{\Gamma} \nu_{\Gamma}: \nabla_{\Gamma} \nu_{\Gamma}\right)
$$

Proof: We omit the proof of the equations (2.7) and (2.8). The derivation is elementary but in parts lengthy. The variation of the surface area and unit normal field have been dealt with elsewhere quite intensively, see for example [NiTsCHE 1975].

Equation (2.9) is clear because $\psi$ has no tangential components:

$$
\begin{aligned}
\nabla_{\Gamma} \cdot \psi & =\nabla_{\Gamma} \cdot\left(\left\langle\psi, \nu_{\Gamma}\right\rangle \nu_{\Gamma}\right) \\
& =\left\langle\psi, \nu_{\Gamma}\right\rangle \nabla_{\Gamma} \cdot \nu_{\Gamma}+\underbrace{\left\langle\left(\nabla_{\Gamma} \psi\right) \cdot \nu_{\Gamma}, \nu_{\Gamma}\right\rangle}_{=0}+\underbrace{\left\langle\left(\nabla_{\Gamma} \nu_{\Gamma}\right) \cdot \psi, \nu_{\Gamma}\right\rangle}_{=0} \\
& =\left\langle\psi, \nu_{\Gamma}\right\rangle 2 H_{\Gamma}=\left\langle\psi, Y_{\Gamma}\right\rangle .
\end{aligned}
$$


Equation (2.10) is a simple application of the product rule:

$$
0=\nu_{\Gamma} \cdot \nabla_{\Gamma} \nu_{\Gamma}=\nabla_{\Gamma} \cdot\left(\nu_{\Gamma} \cdot \nabla_{\Gamma} \nu_{\Gamma}\right)=\nabla_{\Gamma} \nu_{\Gamma}: \nabla_{\Gamma} \nu_{\Gamma}+\nu_{\Gamma} \Delta_{\Gamma} \nu_{\Gamma} .
$$

It is clear that $\nabla_{\Gamma} \nu_{\Gamma}: \nabla_{\Gamma} \nu_{\Gamma}=\kappa_{1}^{2}+\kappa_{2}^{2}$ because $\nabla_{\Gamma} \nu_{\Gamma}$ can be diagonalised in each point of $\Gamma$.

Equation (2.11) follows from the chain-rule and by exploiting orthogonality relations:

$$
\begin{aligned}
\nu_{\Gamma} \cdot \Delta_{\Gamma} \psi & =\nu_{\Gamma} \cdot\left(\Delta_{\Gamma}\left(\bar{\psi} \nu_{\Gamma}\right)\right)=\nu_{\Gamma} \cdot\left(\nu_{\Gamma} \Delta_{\Gamma} \bar{\psi}+\bar{\psi} \Delta_{\Gamma} \nu_{\Gamma}+2 \nabla_{\Gamma} \nu_{\Gamma} \cdot \nabla_{\Gamma} \bar{\psi}\right) \\
& =\Delta_{\Gamma} \bar{\psi}+\bar{\psi} \nu_{\Gamma} \cdot \Delta_{\Gamma} \nu_{\Gamma}+2 \underbrace{\nu_{\Gamma} \cdot \nabla_{\Gamma} \nu_{\Gamma}}_{=0} \cdot \nabla_{\Gamma} \bar{\psi} \stackrel{(2.10)}{=} \Delta_{\Gamma} \bar{\psi}-\bar{\psi}\left(\nabla_{\Gamma} \nu_{\Gamma}: \nabla_{\Gamma} \nu_{\Gamma}\right) .
\end{aligned}
$$

We will now employ Lemma 2.1.1 on the facing page to derive an expression for the second variation of the drop energy. For $\varphi, \psi \in\left(\mathrm{C}^{k, \alpha}(\Gamma)\right)^{3}$ and $K, J \in \mathbb{R}$ the second variation $\delta^{2} E(\Gamma, C)$ of $E$ at $(\Gamma, C)$ in direction of $(\varphi, K)$ and $(\psi, J)$ is

$\delta^{2} E(\Gamma, C)[\varphi, K][\psi, J]=\delta(\delta E(\Gamma, C)[\varphi, K])[\psi, J]=\left.\frac{d}{d \varepsilon}\left(\delta E\left(\Gamma_{\psi}(\varepsilon), C+\varepsilon J\right)[\varphi, K]\right)\right|_{\varepsilon=0}$.

Second variation $\boldsymbol{\delta}^{2} \boldsymbol{E}_{\text {surf }}$ of the surface energy: As a consequence of (2.8) on the preceding page we have:

$$
\begin{aligned}
\delta^{2} E_{\text {surf }}(\Gamma)[\varphi][\psi]= & \left.\frac{d}{d \varepsilon}\left(-\int_{\Gamma_{\psi}(\varepsilon)} \Delta_{\Gamma_{\psi}(\varepsilon)} \operatorname{id}_{\Gamma_{\psi}(\varepsilon)} \cdot \varphi d o\right)\right|_{\varepsilon=0} \\
= & -\left.\int_{\Gamma} \frac{d}{d \varepsilon}\left(\Delta_{\Gamma_{\psi}(\varepsilon)} \mathrm{id}_{\Gamma_{\psi}(\varepsilon)}\right)\right|_{\varepsilon=0} \cdot \varphi d o-\int_{\Gamma} \Delta_{\Gamma} \mathrm{id}_{\Gamma} \cdot \varphi \nabla_{\Gamma} \cdot \psi d o \\
\stackrel{(2.8)}{=} & -\int_{\Gamma}\left(\nu_{\Gamma} \nu_{\Gamma} \cdot \Delta_{\Gamma} \psi+2 \nu_{\Gamma} \nabla_{\Gamma} \psi: \nabla_{\Gamma} \nu_{\Gamma}+\left(\nabla_{\Gamma} \psi\right)^{\mathrm{tr}} \cdot Y_{\Gamma}\right) \cdot \varphi d o \\
& -\int_{\Gamma} \Delta_{\Gamma} \mathrm{id}_{\Gamma} \cdot \varphi \psi \cdot Y_{\Gamma} d o \\
= & -\int_{\Gamma} \varphi \cdot \Delta_{\Gamma} \psi+2 \varphi \cdot \nu_{\Gamma} \nabla_{\Gamma} \psi: \nabla_{\Gamma} \nu_{\Gamma}+\underbrace{\varphi_{i} \delta_{i} \psi_{j}}_{=0}\left(Y_{\Gamma}\right)_{j} d o \\
& -\int_{\Gamma} \Delta_{\Gamma} \mathrm{id}_{\Gamma} \cdot \varphi \psi \cdot Y_{\Gamma} d o \\
= & -\int_{\Gamma} \psi \cdot \Delta_{\Gamma} \varphi d o-2 \underbrace{\int_{\Gamma} \varphi \cdot \nu_{\Gamma} \nabla_{\Gamma} \psi: \nabla_{\Gamma} \nu_{\Gamma} d o}_{=: I_{1}} \\
& -\int_{\Gamma} \psi \cdot Y_{\Gamma} \Delta_{\Gamma} \mathrm{id}_{\Gamma} \cdot \varphi d_{o}
\end{aligned}
$$

$I_{1}$ can be transformed using integration by parts:

$$
\begin{aligned}
I_{1} & =\int_{\Gamma} \varphi \cdot \nu_{\Gamma} \nabla_{\Gamma} \psi: \nabla_{\Gamma} \nu_{\Gamma} d o \\
& =-\int_{\Gamma} \underbrace{\psi \cdot \nabla_{\Gamma} \nu_{\Gamma}}_{=0} \nabla_{\Gamma}\left(\varphi \cdot \nu_{\Gamma}\right) d o-\int_{\Gamma} \varphi \cdot \nu_{\Gamma} \psi \cdot \Delta_{\Gamma} \nu_{\Gamma} d o .
\end{aligned}
$$


Substituting (2.13) in (2.12) and using equation (2.10) from Lemma 2.1.1 on page 24 we arrive at the following expression of the second variation of the surface energy:

$$
\begin{aligned}
\delta^{2} E_{\text {surf }}(\Gamma)[\varphi][\psi]= & -\int_{\Gamma} \psi \cdot \Delta_{\Gamma} \varphi d o-2 \int_{\Gamma} \psi \cdot \nu_{\Gamma} \varphi \cdot \nu_{\Gamma}\left(\nabla_{\Gamma} \nu_{\Gamma}: \nabla_{\Gamma} \nu_{\Gamma}\right) d o \\
& +\int_{\Gamma} \psi \cdot Y_{\Gamma} Y_{\Gamma} \cdot \varphi d o
\end{aligned}
$$

It follows from (2.11) on page 24 that (2.14) depends only on the scalar functions $\bar{\varphi}=\varphi \cdot \nu_{\Gamma}$ and $\bar{\psi}=\psi \cdot \nu_{\Gamma}$ :

$$
\begin{aligned}
\delta^{2} E_{\mathrm{surf}}(\Gamma)[\varphi][\psi]= & -\int_{\Gamma} \bar{\psi} \nu_{\Gamma} \cdot \Delta_{\Gamma}\left(\bar{\varphi} \nu_{\Gamma}\right) d o-2 \int_{\Gamma} \bar{\psi} \bar{\varphi}\left(\nabla_{\Gamma} \nu_{\Gamma}: \nabla_{\Gamma} \nu_{\Gamma}\right) d o \\
& -\int_{\Gamma} \bar{\psi} 2 H_{\Gamma} \bar{\varphi} 2 H_{\Gamma} d o \\
= & -\int_{\Gamma} \bar{\psi} \Delta_{\Gamma} \bar{\varphi} d o-\int_{\Gamma} \bar{\psi} \bar{\varphi}\left(\nabla_{\Gamma} \nu_{\Gamma}: \nabla_{\Gamma} \nu_{\Gamma}\right) d o \\
& -\int_{\Gamma} 2 H_{\Gamma}\left(\bar{\psi} \bar{\varphi} 2 H_{\Gamma}\right) d o .
\end{aligned}
$$

\section{Second variation $\delta^{2} E_{\text {rot }}$ of the rotational energy:}

$$
\begin{aligned}
\delta^{2} E_{\mathrm{rot}}(\Gamma)[\varphi][\psi] & =\left.\frac{d}{d \varepsilon} \delta E_{\mathrm{rot}}\left(\Gamma_{\psi}(\varepsilon)\right)[\varphi]\right|_{\varepsilon=0} \\
& =-\left.4 \omega^{2} \frac{d}{d \varepsilon}\left(\int_{\Gamma_{\psi}(\varepsilon)} r_{\varepsilon}^{2} \nu_{\Gamma_{\psi}(\varepsilon)} \cdot \varphi d o\right)\right|_{\varepsilon=0} \\
& =-4 \omega^{2}\left(\left.\int_{\Gamma} \varphi \cdot \frac{d}{d \varepsilon}\left(r_{\varepsilon}^{2} \nu_{\Gamma_{\psi}(\varepsilon)}\right)\right|_{\varepsilon=0} d o+\int_{\Gamma} r^{2} \nu_{\Gamma} \cdot \varphi \nabla_{\Gamma} \cdot \psi d o\right),
\end{aligned}
$$

with

$$
r_{\varepsilon}^{2}:=\left(x_{1}+\varepsilon \psi_{1}\right)^{2}+\left(x_{2}+\varepsilon \psi_{2}\right)^{2}=r^{2}+2 \varepsilon\left(x_{1} \psi_{1}+x_{2} \psi_{2}\right)+o\left(\varepsilon^{2}\right) .
$$

and $\operatorname{id}_{\Gamma}=\left(x_{1}, x_{2}, x_{3}\right), \psi=\left(\psi_{1}, \psi_{2}, \psi_{3}\right)$.

Inserting the above relation and (2.7) (variation of the normal field) from Lemma 2.1.1 on page 24 into (2.16) above we get

$$
\begin{aligned}
\delta^{2} E_{\mathrm{rot}}(\Gamma)[\varphi][\psi]= & -4 \omega^{2}(\int_{\Gamma} 2 \varphi \cdot \nu_{\Gamma}\left(x_{1} \psi_{1}+x_{2} \psi_{2}\right) d o-\int_{\Gamma} r^{2} \underbrace{\varphi \cdot\left(\nabla_{\Gamma} \psi\right)^{\mathrm{tr}}}_{=0} \cdot \nu_{\Gamma} d o) \\
& -4 \omega^{2} \int_{\Gamma} r^{2} \nu_{\Gamma} \cdot \varphi \nabla_{\Gamma} \cdot \psi d o .
\end{aligned}
$$


Using $\psi=\left(\psi \cdot \nu_{\Gamma}\right) \nu_{\Gamma}$ we finally arrive at the following expressions:

$$
\begin{aligned}
\delta^{2} E_{\mathrm{rot}}(\Gamma)[\varphi][\psi]= & -8 \omega^{2} \int_{\Gamma} \varphi \cdot \nu_{\Gamma} \psi \cdot \nu_{\Gamma}\left(x_{1} \nu_{1}+x_{2} \nu_{2}\right) d o \\
& -4 \omega^{2} \int_{\Gamma} \psi \cdot Y_{\Gamma} r^{2} \nu_{\Gamma} \cdot \varphi d o \\
\Longleftrightarrow \quad \delta^{2} E_{\mathrm{rot}}(\Gamma)[\varphi][\psi]= & -8 \omega^{2} \int_{\Gamma} \bar{\varphi} \bar{\psi}\left(x_{1} \nu_{1}+x_{2} \nu_{2}\right) d o-4 \omega^{2} \int_{\Gamma} 2 H_{\Gamma} \bar{\psi} \bar{\varphi} r^{2} d o .
\end{aligned}
$$

\section{Second variation $\delta^{2} E_{\mathrm{vol}}$ of the volume constraint:}

$$
\begin{aligned}
& \delta^{2} E_{\mathrm{vol}}(\Gamma, C)[\varphi, K][\psi, J]=\left.\frac{d}{d \varepsilon}\left(\delta E_{\mathrm{vol}}\left(\Gamma_{\psi}(\varepsilon), C+\varepsilon J\right)[\varphi, K]\right)\right|_{\varepsilon=0} \\
& =-\left.\frac{d}{d \varepsilon}\left((C+\varepsilon J) \int_{\Gamma_{\psi}(\varepsilon)} \varphi \cdot \nu_{\Gamma_{\psi}(\varepsilon)} d o+K\left(\int_{\Gamma_{\psi}(\varepsilon)} \operatorname{id}_{\Gamma_{\psi}(\varepsilon)} \cdot \nu_{\Gamma_{\psi}(\varepsilon)} d o-4 \pi\right)\right)\right|_{\varepsilon=0} .
\end{aligned}
$$

Taking into account that the variation of the normal field is tangential to $\Gamma$ whereas $\psi$ and $\varphi$ are orthogonal variations the expressions for the second variation of the volume constraint are

$$
\begin{gathered}
\delta^{2} E_{\mathrm{vol}}(\Gamma, C)[\varphi, K][\psi, J]=-J \int_{\Gamma} \varphi \cdot \nu_{\Gamma} d o-K \int_{\Gamma} \psi \cdot \nu_{\Gamma} d o-C \int_{\Gamma} Y_{\Gamma} \cdot \psi \nu_{\Gamma} \cdot \varphi d o \\
(2.20) \Longleftrightarrow \delta^{2} E_{\mathrm{vol}}(\Gamma, C)[\varphi, K][\psi, J]=-J \int_{\Gamma} \bar{\varphi} d o-K \int_{\Gamma} \bar{\psi} d o-C \int_{\Gamma} 2 H_{\Gamma} \bar{\psi} \bar{\varphi} d o .
\end{gathered}
$$

By summing up the different expressions for the second variation of $E_{\text {surf }}, E_{\text {rot }}$ and $E_{\mathrm{vol}}$ given in the equations $(2.14),(2.17)$ and (2.19) (respectively (2.15), (2.18) and (2.20) for the scalar case) we finally arrive at an expression for the second variation $\delta^{2} E(\Gamma, C)[\varphi, K][\psi, J]$ of $E(\Gamma, C)$ at $(\varphi, K)$ and $(\psi, J)$ :

$$
\begin{aligned}
\delta^{2} E[\varphi, K][\psi, J]= & -\int_{\Gamma} \psi \cdot \Delta_{\Gamma} \varphi d o-2 \int_{\Gamma} \psi \cdot \nu_{\Gamma} \varphi \cdot \nu_{\Gamma}\left(\nabla_{\Gamma} \nu_{\Gamma}: \nabla_{\Gamma} \nu_{\Gamma}\right) d o \\
& -8 \omega^{2} \int_{\Gamma} \varphi \cdot \nu_{\Gamma} \psi \cdot \nu_{\Gamma}\left(x_{1} \nu_{1}+x_{2} \nu_{2}\right) d o \\
& -J \int_{\Gamma} \varphi \cdot \nu_{\Gamma} d o-K \int_{\Gamma} \psi \cdot \nu_{\Gamma} d o \\
& +\int_{\Gamma} Y_{\Gamma} \cdot \psi \varphi \cdot \underbrace{\left(Y_{\Gamma}-\left(4 \omega^{2} r^{2}+C\right) \nu_{\Gamma}\right)}_{\equiv 0 \text { if }(\Gamma, C) \text { is a solution of }(2.6)} d o .
\end{aligned}
$$


Using $\bar{\varphi}=\varphi \cdot \nu_{\Gamma}$ and $\bar{\psi}=\psi \cdot \nu_{\Gamma}$ this can be written in scalar terms:

$$
\begin{aligned}
\delta^{2} E[\varphi, K][\psi, J]= & -\int_{\Gamma} \bar{\psi} \Delta_{\Gamma} \bar{\varphi} d o-\int_{\Gamma} \bar{\psi} \bar{\varphi}\left(\nabla_{\Gamma} \nu_{\Gamma}: \nabla_{\Gamma} \nu_{\Gamma}\right) d o \\
& -8 \omega^{2} \int_{\Gamma} \bar{\varphi} \bar{\psi}\left(x_{1} \nu_{1}+x_{2} \nu_{2}\right) d o \\
& -J \int_{\Gamma} \bar{\varphi} d o-K \int_{\Gamma} \bar{\psi} d o \\
& +\int_{\Gamma} 2 H_{\Gamma} \bar{\psi} \bar{\varphi} \underbrace{\left(2 H_{\Gamma}-4 \omega^{2} r^{2}-C\right)}_{\equiv 0 \text { if }(\Gamma, C) \text { is a solution of }(2.6)} d o .
\end{aligned}
$$

We remark that the residual $R_{(\Gamma, C, \omega)}:=2 H_{\Gamma}-\left(4 \omega^{2} r^{2}+C\right)$ vanishes if the second variation is evaluated at a solution $(\Gamma, C)$ of the non-linear Euler-Lagrange equation (2.6). So the last integral in (2.21) and (2.22) is zero in this case.

The linearisations of the non-linear Euler-Lagrange equatio (2.6) are the Euler-Lagrange equations implied by the expressions $(2.21)$ and (2.22) for the second variation $\delta^{2} E$, evaluated at a solution $(\Gamma, C)$. The non-linear Euler-Lagrange equation (2.6) is repeated here for clarity.

$$
\begin{gathered}
-\Delta_{\Gamma} \mathrm{id}_{\Gamma}=\left(4 \omega^{2} r^{2}+C\right) \nu_{\Gamma}, \quad \int_{\Gamma} \operatorname{id}_{\Gamma} \cdot \nu_{\Gamma} d o=4 \pi . \\
\left\{\begin{array}{l}
-\Delta_{\Gamma} \varphi-2 \nu_{\Gamma}\left(\kappa_{1}^{2}+\kappa_{2}^{2}\right) \nu_{\Gamma} \cdot \varphi-8 \omega^{2} \nu_{\Gamma}\left(x_{1} \nu_{1}+x_{2} \nu_{2}\right) \nu_{\Gamma} \cdot \varphi-K \nu_{\Gamma}=0, \\
\int_{\Gamma} \varphi \cdot \nu_{\Gamma} d o=0 .
\end{array}\right. \\
-\Delta_{\Gamma} \bar{\varphi}-\nu_{\Gamma}\left(\kappa_{1}^{2}+\kappa_{2}^{2}\right) \bar{\varphi}-8 \omega^{2}\left(x_{1} \nu_{1}+x_{2} \nu_{2}\right) \bar{\varphi}-K=0, \quad \int_{\Gamma} \bar{\varphi} d o=0 .
\end{gathered}
$$

We note that (2.23) is a coupled system of partial differential equations for the components of the orthogonal perturbation $\varphi$ while (2.24) is a single partial differential equation for the signed length $\bar{\varphi}$ of $\varphi$.

\subsection{The linear Euler-Lagrange equations}

As a preparation for the continuous (in the sense of non-discrete) Newton method described in Section 2.3 below we continue by examining the linear Euler-Lagrange equations (2.23) and (2.24) for the non-homogeneous case. For brevity we define

$$
\begin{aligned}
& a_{\Gamma}:=8 \omega^{2}\left(x_{1} \nu_{1}+x_{2} \nu_{2}\right)+2\left(\kappa_{1}^{2}+\kappa_{2}^{2}\right) \\
& \bar{a}_{\Gamma}:=8 \omega^{2}\left(x_{1} \nu_{1}+x_{2} \nu_{2}\right)+\left(\kappa_{1}^{2}+\kappa_{2}^{2}\right) .
\end{aligned}
$$

Note that $\bar{a}_{\Gamma}$ is not equal to $a_{\Gamma}$ and - violating the conventions introduced above $-a_{\Gamma}$ and $\bar{a}_{\Gamma}$ are both scalar quantities.

$\Gamma$ is still assumed to be of class $\mathrm{C}^{k, \alpha}, k \geq 3$, so $a_{\Gamma}$ and $\bar{a}_{\Gamma}$ are of class $\mathrm{C}^{k-2, \alpha}$. We are interested in solutions $(\varphi, K)$ with $\varphi \in \mathrm{C}^{k, \alpha}(\Gamma), K \in \mathbb{R}$. 


$$
\begin{gathered}
-\Delta_{\Gamma} \varphi-a_{\Gamma} \nu_{\Gamma} \cdot \varphi \nu_{\Gamma}-K \nu_{\Gamma}=f, \quad \int_{\Gamma} \varphi \cdot \nu_{\Gamma} d o=b . \\
-\Delta_{\Gamma} \bar{\varphi}-\bar{a}_{\Gamma} \bar{\varphi}-K=\bar{f}, \quad \int_{\Gamma} \bar{\varphi} d o=b .
\end{gathered}
$$

For the right hand side $(f, b)$ we assume that $f \in \mathrm{C}^{k-2, \alpha}(\Gamma)$ and $b \in \mathbb{R}$ is just a real number. When used in the continuous Newton method below $(f, b)$ will be the residual of the non-linear Euler-Lagrange equations (2.6). So later we will have $f=R_{(\Gamma, C, \omega)} \nu_{\Gamma}$, $b=\int_{\Gamma} \mathrm{id}_{\Gamma} \cdot \nu_{\Gamma} d o-4 \pi$.

REMARK 2.2.1 (2.26) and (2.27) have the structure of a Neumann-problem. The Fredholm alternative - which is not quoted in this work - defines compatibility conditions for the right-hand side in order that (2.26) and (2.27) have a solution, see for example [Evans 1998, Appendix D.5].

Specifically, it is necessary for the scalar equation $(2.27)$ that $\bar{f}+K \in N\left(\left(-\Delta_{\Gamma}-\bar{a}_{\Gamma}\right)^{*}\right)^{\perp}$, where $N(\cdot)$ denotes the kernel of a linear operator and $V^{\perp}$ the orthogonal complement of a Hilbert (sub-)space. $A^{*}$ is the adjoint of a bounded linear operator $A$ on a Hilbert space.

This determines the value of $K$. However, the Fredholm alternative does not provide an explicit expression for $K$ in this general setup.

The problems (2.26) and (2.27) are ill-posed in the sense that we do not have uniqueness of a solution. Therefore we continue by examining the Lie-symmetries of the non-linear Euler-Lagrange equation (2.6) and their consequences for its linearisation.

\subsubsection{Lie-symmetries}

When omitting the volume constraint the non-linear Euler-Lagrange equation (2.6) is invariant with respect to the one-parameter groups of scalings, rotations about the axis of rotation and translations in direction of the axis of rotation. The three symmetries span a 3-dimensional Lie-group which maps any solution of (2.6) to another solution of (2.6).

Thus any solution of (2.6) which is not invariant with respect to this Lie-group generates a differential manifold of solutions. As a consequence, the homogeneous linearised equations have non-trivial solutions in this case.

Scalings: The invariance with respect to scalings is broken by imposing the volume constraint, so we need not discuss it further. We refer here to the remark on page 4 at the end of Section 1.1. 
Translations in $\boldsymbol{x}_{\boldsymbol{3}}$-direction: The invariance with respect to translations in direction of the axis of rotation could be dealt with in a like-wise manner: we just could impose the constraint that the centre of mass remains at the origin and deduce from this a linearised centre-of-mass constraint in the same way we deduced the linearised volume constraint. It is - maybe - remarkable that the homogeneous linearisation just ends up in $\int_{\Gamma} \bar{\varphi} x_{3} d o=0$.

We will omit the proof because we do not need this result. Instead, we use the following theorem to break the translational invariance in $x_{3}$-direction:

\subsubsection{Theorem (Equatorial Reflective Symmetry, [Wente 1982])}

A solution $\Gamma$ of class $\mathrm{C}^{2}$ of (2.6) owns a reflective symmetry with respect to the $\left(x_{1}, x_{2}\right)$ plane, where the $x_{3}$-axis is the axis of rotation.

Proof: The reference to Wente's publication which contains the proof is in the heading of the theorem above. We just like to remark that in his work Wente also takes selfgravitation into account, proving the result stated in the theorem in a far more general regime.

Wente's theorem means that we can eliminate the translational invariance in the $x_{3^{-}}$ direction by cutting the drop at its equatorial plane and imposing Dirichlet boundary conditions on the $x_{3}$-component of the solutions of (2.6) and Neumann boundary conditions on the $x_{1}$ and $x_{2}$ components. We remark that by Theorem 2.2.1 the attribute "equatorial" is well-defined.

REMARK 2.2.2 Cutting the drop at its equatorial plane is just a matter of a clever treatment of (2.6) and its linearisations (2.26) and (2.27). It has no effect on existence and regularity of solutions for the equations above (although - by construction - it has a serious effect on the uniqueness).

In the discussion of the regularity of the solutions of the linearisations of (2.6) we will not dissect the drop at its equatorial plane but instead use reflectively symmetric Ansatzfunctions, see Definition 2.2.2 on page 32.

In the treatment of the discretisation of the Newton scheme the boundary conditions will be used explicitly, because the Theorem 2.2.1 saves half of the computation time. We will, nevertheless, not consistently use definitions like $\Gamma^{+}$for the "upper" half of the drop-surface and so on. We will rather comment on the regime in use at the start of each section for the remainder of our analysis.

Axial symmetry about the $\boldsymbol{x}_{\mathbf{3}}$-axis The invariance of the non-linear EulerLagrange equations (2.6) under the action of a one-parameter Lie-group $G$ implies that the image of a solution $(\Gamma, C)$ under the infinitesimal generator $\mathcal{G}$ of $G$ is a solution of the linearised equations. $\mathcal{G} \cdot(\Gamma, C)$ will be a non-trivial solution if $(\Gamma, C)$ is not invariant under the action of $G$.

While this is clear we want to establish the fact explicitly for the case of the oneparameter group $R$ of rotations around the $x_{3}$-axis, the axis of rotation. As subgroup of 
$G L(3, \mathbb{R})$ we have the following presentation for $R$ and its infinitesimal generator $\mathcal{R}$ :

$$
G=\left\{g(\varepsilon):=\left(\begin{array}{ccc}
\cos \varepsilon & -\sin \varepsilon & 0 \\
\sin \varepsilon & \cos \varepsilon & 0 \\
0 & 0 & 1
\end{array}\right) \mid \varepsilon \in \mathbb{R}\right\}, \quad \mathcal{R}=\left(\begin{array}{ccc}
0 & -1 & 0 \\
1 & 0 & 0 \\
0 & 0 & 0
\end{array}\right) .
$$

The canonical action of the Group $G$ on a solution $(\Gamma, C)$ of the non-linear Euler-Lagrange equation generates a differentiable family of solutions $G \cdot(\Gamma, C):=\{(g(\varepsilon) \cdot \Gamma, C) \mid \varepsilon \in \mathbb{R}\}$. We can expand the mapping $\varepsilon \mapsto g(\varepsilon) \cdot \Gamma$ into a Taylor-series around $\varepsilon=0$ because $G$ is a Lie-group. Up to first order in $\varepsilon$ we thus arrive at $g(\varepsilon) \cdot \Gamma=\Gamma+\varepsilon \mathcal{R} \cdot \Gamma+o\left(\varepsilon^{2}\right)$.

This induces an orthogonal perturbation $\Theta:=\left\langle\mathcal{R} \cdot \operatorname{id}_{\Gamma}, \nu_{\Gamma}\right\rangle \nu_{\Gamma} \in \mathrm{C}^{k-1, \alpha}(\Gamma)$ which is - by construction - a solution of the linearised Euler-Lagrange equation (2.23). We have at a point $X=\left(x_{1}, x_{2}, x_{3}\right) \in \Gamma$ that

$$
\Theta(X)=(\mathcal{R} \cdot X) \nu_{\Gamma}=\left(-x_{2} \nu_{1}+x_{1} \nu_{2}\right) \nu_{\Gamma} .
$$

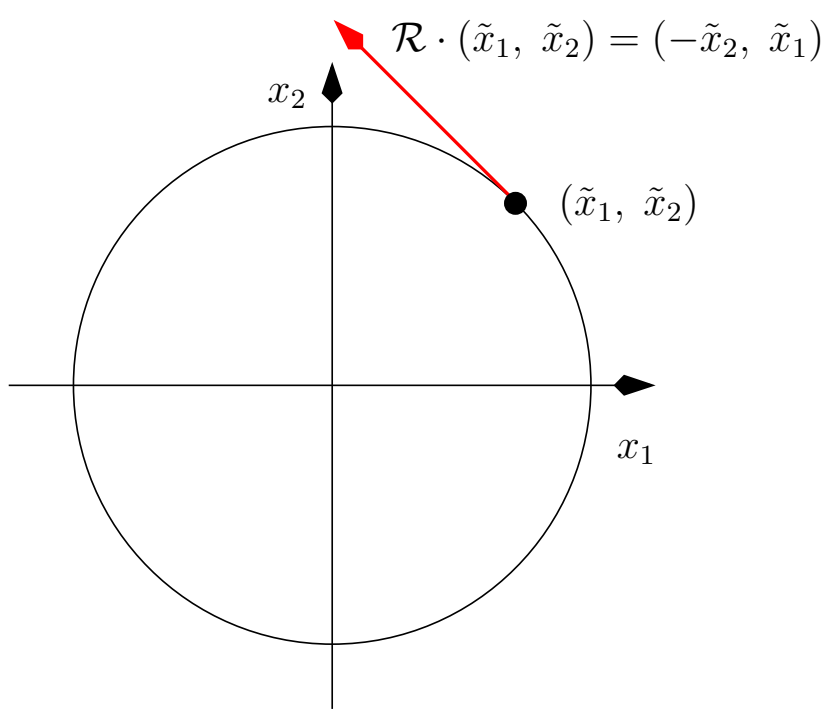

So clearly $\Theta \equiv 0$ if and only if $\Gamma$ is invariant under the action of $G$. We note that the same holds for $\bar{\Theta}(X)=\left(-x_{2} \nu_{1}+x_{1} \nu_{2}\right)$ with respect to the scalar version $(2.24)$ of the linearisation.

Figure 2.1 The action of an infinitesimal rotation

This means that in the presence of symmetry breaking - if $\Gamma$ is not axially symmetric - we can eliminate the singularity of the second variation $\delta^{2} E(\Gamma, C)$ by allowing only perturbations which are transversal to $\Theta$ respectively to $\bar{\Theta}$ in the scalar case.

The notion of "transversal" will be made explicit in the next section when we define weak solutions for the linearised Euler-Lagrange equations (2.23) and (2.24).

We remark at this point that earlier numerical methods for the computation of equilibrium shapes of rotating drops employed by others - the work of [BROWN and SCRIVEN 1980a] being the one of greatest importance - were imposing an artificial reflective symmetry about a meridional plane of the drop surface. The boundary conditions implied by dissecting the drop at this plane also eliminate the singularity of the second variation $\delta^{2} E(\Gamma, C)$. We do not use this technique because we do not want to impose artificial symmetry constraints.

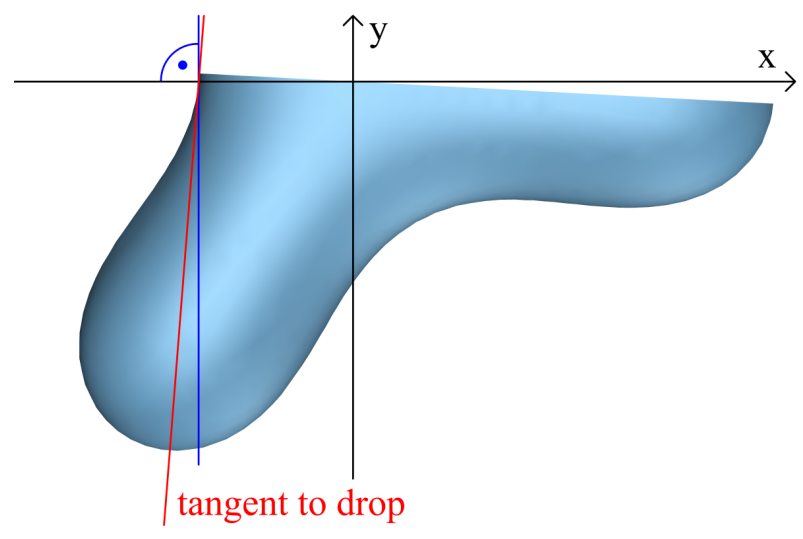

Figure 2.2 Rotations violate the Neumann boundary conditions implied by an artificial dissection at a meridional plane 


\subsubsection{Weak solutions of the linearisation}

We are going to define the notion of a weak solution for both the vector-valued and the scalar-valued non-homogeneous linearised Euler-Lagrange equations defined in (2.26) and (2.27) on page 29. The most important step is choosing an appropriate function space which will contain the weak solutions.

In the following we lower the differentiability-class for the surface $\Gamma$ to $\mathrm{C}^{k, \alpha}$ with $k \geq 2$ (we previously required $k \geq 3$ ). More specifically in the following we assume that $\Gamma \subset \mathbb{R}^{3}$ is an isometrically immersed compact surface of class $\mathrm{C}^{k, \alpha}$ with $k \geq 2$ and $0<\alpha<1$. And we assume that $\Gamma$ possesses a reflective symmetry about the $\left(x_{1}, x_{2}\right)$-plane.

Therefore we can decompose $\Gamma$ as follows:

$$
\Gamma^{+}:=\Gamma \cap\left(\mathbb{R}^{2} \times \mathbb{R}^{+}\right), \quad \Gamma^{-}:=\Gamma \cap\left(\mathbb{R}^{2} \times \mathbb{R}^{-}\right), \quad \Sigma:=\partial \Gamma^{+} \equiv \partial \Gamma^{-} .
$$

Let $\mathcal{I}: \mathbb{R}^{3} \rightarrow \mathbb{R}^{3}:\left(x_{1}, x_{2}, x_{3}\right) \mapsto\left(x_{1}, x_{2},-x_{3}\right)$ denote the reflection about the $\left(x_{1}, x_{2}\right)$ plane. We obviously have

$$
\Gamma=\Gamma^{+} \cup \Sigma \cup \Gamma^{-} \text {and } \Gamma^{-}=\mathcal{I} \cdot \Gamma^{+} .
$$

In preparation for the definition of weak solutions we introduce the following notations:

\subsubsection{Definition (Function Spaces for the Weak Solutions)}

Under the assumption listed above we define the following function spaces on $\Gamma$ :

reflectively symmetric scalar functions

$$
\underline{W}_{ \pm}(\Gamma):=\left\{v \in \mathrm{H}^{1}(\Gamma) \mid v(X)=v(\mathcal{I} \cdot X) \quad \forall X \in \Gamma\right\} .
$$

reflectively symmetric vector-valued functions

$$
W_{ \pm}(\Gamma):=\left\{\left(v_{1}, v_{2}, v_{3}\right) \in\left(\mathrm{H}^{1}(\Gamma)\right)^{3} \mid v_{3}(X)=-v_{3}(\mathcal{I} \cdot X) \quad \forall X \in \Gamma\right\} .
$$

axially asymmetric scalar functions

$$
\underline{W}_{\circlearrowleft}(\Gamma):=\left\{v \in \mathrm{H}^{1}(\Gamma) \mid \int_{\Gamma} \bar{\Theta} v d o=0\right\},
$$

where $\bar{\Theta}$ is the action of the infinitesimal generator of the group of rotations about the $x_{3}$-axis, as defined in the previous section, that is $\bar{\Theta}=-x_{2} \nu_{1}+x_{1} \nu_{2}$ for $X=\left(x_{1}, x_{2}, x_{3}\right) \in \Gamma$.

\section{axially asymmetric vector-valued functions}

$$
W_{\circlearrowleft}(\Gamma):=\left\{v \in\left(\mathrm{H}^{1}(\Gamma)\right)^{3} \mid \int_{\Gamma} \Theta \cdot v d o=0\right\}
$$

where $\Theta$ is the action of the infinitesimal generator of the group of rotations about the $x_{3}$-axis, as defined in the previous section, that is $\Theta=\left(-x_{2} \nu_{1}+x_{1} \nu_{2}\right) \nu_{\Gamma}$ for $X=\left(x_{1}, x_{2}, x_{3}\right) \in \Gamma$. 


\section{scalar mean-value constraint}

$$
\underline{W}(\Gamma):=\left\{v \in \mathrm{H}^{1}(\Gamma) \mid \int_{\Gamma} v d o=0\right\} .
$$

We note that any function $\tilde{v}$ in the affine linear space $\underline{W}^{b}(\Gamma):=\underline{W}(\Gamma)+\frac{b}{|\Gamma|}$ satisfies the non-homogeneous volume constraint $\int_{\Gamma} \tilde{v} d o=b, b \in \mathbb{R}$.

\section{vector-valued mean-value constraint}

$$
W(\Gamma):=\left\{v \in\left(\mathrm{H}^{1}(\Gamma)\right)^{3} \mid \int_{\Gamma} v \cdot \nu_{\Gamma} d o=0\right\} .
$$

We note that any function $\tilde{v}$ in the affine linear space $W^{b}(\Gamma):=W(\Gamma)+\frac{b}{|\Gamma|} \nu_{\Gamma}$ satisfies the non-homogeneous volume constraint $\int_{\Gamma} \tilde{v} d o=b, b \in \mathbb{R}$.

\section{orthogonal weak vector-fields}

$$
W^{\perp}(\Gamma):=\left\{v \in\left(\mathrm{H}^{1}(\Gamma)\right)^{3} \mid\left\langle v, \nu_{\Gamma}\right\rangle \nu_{\Gamma}=v\right\} .
$$

We note that $W^{\perp}(\Gamma)$ is well defined because $\Gamma$ is of class $\mathrm{C}^{k, \alpha}$ and so $\nu_{\Gamma} \in \mathrm{C}^{k-1, \alpha}(\Gamma)$ holds $(k \geq 2)$.

\subsubsection{LEMMA}

Under the assumptions on $\Gamma$ used in Definition 2.2.2 on the facing page it holds:

1. $\underline{W}_{ \pm}(\Gamma), \underline{W}_{\circlearrowleft}(\Gamma)$ and $\underline{W}(\Gamma)$ are closed subspaces of $\mathrm{H}^{1}(\Gamma)$.

$$
\underline{W}(\Gamma):=\underline{W}_{ \pm}(\Gamma) \cap \underline{W}_{\circlearrowleft}(\Gamma) \cap \underline{W}(\Gamma)
$$

is a Hilbert-space. Moreover, for any number $b \in \mathbb{R}$,

$$
\underline{W}(\Gamma)+\frac{b}{|\Gamma|}=\underline{W}_{ \pm}(\Gamma) \cap \underline{W}_{\circlearrowleft}(\Gamma) \cap \underline{W}^{b}(\Gamma) .
$$

2. $W_{ \pm}(\Gamma), W_{\circlearrowleft}(\Gamma), W(\Gamma)$ and $W^{\perp}(\Gamma)$ are closed subspaces of $\left(\mathrm{H}^{1}(\Gamma)\right)^{3}$.

$$
W(\Gamma):=W_{ \pm}(\Gamma) \cap W_{\circlearrowleft}(\Gamma) \cap W^{\perp}(\Gamma) \cap W(\Gamma)
$$

is a Hilbert-space. Moreover, for any number $b \in \mathbb{R}$,

$$
W(\Gamma)+\frac{b}{|\Gamma|} \nu_{\Gamma}=W_{ \pm}(\Gamma) \cap W_{\circlearrowleft}(\Gamma) \cap W^{\perp}(\Gamma) \cap W^{b}(\Gamma) .
$$

3. The "orthogonal lift"

$$
{ }^{\perp}: \mathrm{H}^{1}(\Gamma) \rightarrow W^{\perp}(\Gamma): v \mapsto v \nu_{\Gamma}
$$

is an isomorphism between Hilbert-spaces.

4. If $\Gamma$ is axially symmetric with respect to the $x_{3}$-axis, then it holds that $\underline{W}_{\circlearrowleft}(\Gamma)=\mathrm{H}^{1}(\Gamma)$ and $W_{\circlearrowleft}(\Gamma)=\left(\mathrm{H}^{1}(\Gamma)\right)^{3}$. 


\section{Proof:}

ad 1. $\underline{W}_{ \pm}(\Gamma)$ is the kernel of the operation of the involution $\mathcal{I}$ on $\mathrm{H}^{1}(\Gamma)$ and hence $\underline{W}_{ \pm}(\Gamma)$ is closed. $\underline{W}_{\circlearrowleft}(\Gamma)$ and $\underline{W}(\Gamma)$ are kernels of continuous functionals. In the case of $\underline{W}_{\circlearrowleft}(\Gamma)$ the continuity follows from the fact that $\nu_{\Gamma} \in \mathrm{C}^{k-1, \alpha}(\Gamma), k \geq 2$, which especially means that $\underline{W}_{\circlearrowleft}(\Gamma)$ is well-defined. It follows $\underline{W}(\Gamma):=\underline{W}_{ \pm}(\Gamma) \cap \underline{W}_{\circlearrowleft}(\Gamma) \cap \underline{W}(\Gamma) \leq \mathrm{H}^{1}(\Gamma)$ is a Hilbert-space.

Now, for any constant function $\tilde{b} \in \mathrm{H}^{1}(\Gamma)$ with $\tilde{b} \equiv b, b \in \mathbb{R}$, it is clear that $\tilde{b} \in \underline{W}_{ \pm}(\Gamma) \cap \underline{W}_{\circlearrowleft}(\Gamma)$ because the constant functions on $\Gamma$ share all symmetries with $\Gamma$.

This establishes the relation $\underline{W}(\Gamma)+\frac{b}{|\Gamma|}=\underline{W}_{ \pm}(\Gamma) \cap \underline{W}_{\circlearrowleft}(\Gamma) \cap \underline{W}^{b}(\Gamma)$.

ad 2. For $W_{ \pm}(\Gamma), W_{\circlearrowleft}(\Gamma)$ and $W(\Gamma)$ we can conclude as in the scalar case above. $W^{\perp}(\Gamma)$ is a Hilbert-space because of 3 .

It is clear that $W_{ \pm}(\Gamma) \cap W_{\circlearrowleft}(\Gamma) \cap W^{\perp}(\Gamma)$ contains the unit normal field $\nu_{\Gamma}$ and hence all functions which are constant in normal direction. It therefore holds $W(\Gamma)+\frac{b}{|\Gamma|} \nu_{\Gamma}=W_{ \pm}(\Gamma) \cap W_{\circlearrowleft}(\Gamma) \cap W^{\perp}(\Gamma) \cap W^{b}(\Gamma)$.

ad 3. We note that $\nu_{\Gamma} \in \mathrm{C}^{k-1, \alpha}(\Gamma)$ and hence ${ }^{\perp}$ clearly is well defined because $\Gamma$ is compact. ${ }^{\perp}$ is one-to-one because we have an explicit expression for its inverse; it holds for $v \in \mathrm{H}^{1}(\Gamma), w \in W^{\perp}(\Gamma)$ :

$$
\left(\overline{v^{\perp}}\right) \stackrel{\text { def }}{=}\left\langle v \nu_{\Gamma}, \nu_{\Gamma}\right\rangle=v \quad \text { and } \quad \bar{w}^{\perp} \stackrel{\text { def }}{=}\left\langle w, \nu_{\Gamma}\right\rangle \nu_{\Gamma}=w .
$$

$\perp$ is continuous because $\nabla_{\Gamma} \nu_{\Gamma} \in \mathrm{C}^{k-2, \alpha}(\Gamma), k \geq 2$, is bounded because $\Gamma \subset \mathbb{R}^{3}$ is compact. Therefore we have for $u, v \in \mathrm{H}^{1}(\Gamma)$ :

$$
\begin{aligned}
\left\|(u-v)^{\perp}\right\|_{\left(\mathrm{H}^{1}(\Gamma)\right)^{3}}^{2}= & \int_{\Gamma}|u-v|^{2}\left\|\nu_{\Gamma}\right\|^{2} d o \\
& +\int_{\Gamma}\left\|\nu_{\Gamma}\right\|^{2}\left\|\nabla_{\Gamma}(v-u)\right\|^{2} d o+\int_{\Gamma}|v-u|^{2}\left\|\nabla_{\Gamma} \nu_{\Gamma}\right\|^{2} d o \\
\leq & \|u-v\|_{\mathrm{H}^{1}(\Gamma)}^{2}+\left\|\left(\nabla_{\Gamma} \nu_{\Gamma}\right)^{2}\right\|_{\mathrm{L}_{\infty}(\Gamma)}\|u-v\|_{\mathrm{L}_{2}(\Gamma)}^{2} \\
\leq & \left(1+\left\|\nabla_{\Gamma} \nu_{\Gamma}: \nabla_{\Gamma} \nu_{\Gamma}\right\|_{\mathrm{L}_{\infty}(\Gamma)}\right)\|u-v\|_{\mathrm{H}^{1}(\Gamma)}^{2} .
\end{aligned}
$$

Similarly, ${ }^{-}$(the inverse of $\cdot^{\perp}$ ) is bounded. By the same argument we have for $w, p \in W^{\perp}(\Gamma)$

$$
\|\bar{w}-\bar{p}\|_{\mathrm{H}^{1}(\Gamma)}^{2} \leq\left(1+\left\|\nabla_{\Gamma} \nu_{\Gamma}: \nabla_{\Gamma} \nu_{\Gamma}\right\|_{\mathrm{L}_{\infty}(\Gamma)}\right)\left\|(w-p)^{\perp}\right\|_{\left(\mathrm{H}^{1}(\Gamma)\right)^{3}}^{2}
$$

ad 4. If $\Gamma$ is axially symmetric, then it holds that $\Theta$ and $\bar{\Theta}$ are identically zero.

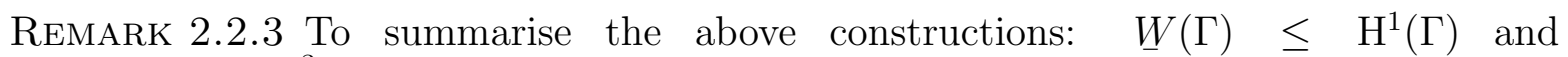
$W(\Gamma) \leq\left(\mathrm{H}^{1}(\Gamma)\right)^{3}$ are well-defined Hilbert-spaces. They relate to the Lie-symmetries of the linearised Euler-Lagrange equations (2.27) and (2.26) on page 29 in that they 
incorporate already the linearised volume constraint, the orthogonality constraint with respect to the infinitesimal generator of the group of rotations about the $x_{3}$-axis and they break the translational invariance in the direction of the $x_{3}$-axis.

This means that $\underline{W}(\Gamma)$ and $W(\Gamma)$ are transversal to the three-dimensional space spanned by the infinitesimal generators of the Lie-symmetry group of the Euler-Lagrange equation. Therefore the non-trivial solutions of the linearised Euler-Lagrange equations induced by the Lie-symmetry group are not contained in the Ansatz-spaces $\underline{W}(\Gamma)$ and $W(\Gamma)$.

\subsubsection{Definition (Weak Solutions of the Linearisation)}

Let $\Gamma \subset \mathbb{R}^{3}$ be an isometrically immersed compact surface of class $\mathrm{C}^{k, \alpha}$ with $k \geq 2$ and $0<\alpha<1$ which possesses a reflective symmetry about the $\left(x_{1}, x_{2}\right)$-plane. Let $f \in\left(\mathrm{C}^{k-2, \alpha}(\Gamma)\right)^{3}, b \in \mathbb{R}$.

1. For $u \in \underline{W}(\Gamma), K \in \mathbb{R}$ we say that $\left(u+\frac{b}{|\Gamma|}, K\right)$ is a weak solution of the linearised Euler-Lagrange equation (2.27) if

$$
\int_{\Gamma} \nabla_{\Gamma} u \cdot \nabla_{\Gamma} \eta d o-\int_{\Gamma} \bar{a}_{\Gamma} u \eta d o=\int_{\Gamma}\left(\bar{f}+\bar{a}_{\Gamma} \frac{b}{|\Gamma|}+K\right) \eta d o
$$

for all $\eta \in \underline{W}(\Gamma)$, where $\bar{a}_{\Gamma}=8 \omega^{2}\left(x_{1} \nu_{1}+x_{2} \nu_{2}\right)+\nabla_{\Gamma} \nu_{\Gamma}: \nabla_{\Gamma} \nu_{\Gamma}$ as defined in (2.25) on page 28.

2. For $w \in W(\Gamma), K \in \mathbb{R}$ we say that $\left(w+\frac{b}{|\Gamma|} \nu_{\Gamma}, K\right)$ is a weak solution of the linearised Euler-Lagrange equation (2.26) if

$$
\int_{\Gamma} \nabla_{\Gamma} w: \nabla_{\Gamma} \chi d o-\int_{\Gamma} a_{\Gamma} \nu_{\Gamma} \cdot w \nu_{\Gamma} \cdot \chi d o=\int_{\Gamma}\left(f+a_{\Gamma} \frac{b}{|\Gamma|} \nu_{\Gamma}+K \nu_{\Gamma}\right) \cdot \chi d o
$$

for all $\chi \in W(\Gamma)$, where $a_{\Gamma}=8 \omega^{2}\left(x_{1} \nu_{1}+x_{2} \nu_{2}\right)+2 \nabla_{\Gamma} \nu_{\Gamma}: \nabla_{\Gamma} \nu_{\Gamma}$ as defined in equation (2.25).

REMARK 2.2.4 We will not analyse existence and uniqueness of solutions of (2.29) and (2.30) above.

\subsubsection{TheOREM (REgularity OF WEAK SOlutions)}

Assume that the prerequisites of Definition 2.2.4 hold.

1. Let $(u, K) \in \underline{W}(\Gamma) \times \mathbb{R}$ be a weak solution of the scalar equation (2.29) in Definition 2.2.4 above. Then it holds that $u \in \mathrm{C}^{k, \alpha}(\Gamma)$.

2. Let $(w, K) \in W(\Gamma) \times \mathbb{R}$ be a weak solution of the vector-valued equation (2.30) in Definition 2.2.4 above. Then it holds that $w \in\left(\mathrm{C}^{k, \alpha}(\Gamma)\right)^{3}$.

Proof: First, let $(u, K) \in \underline{W}(\Gamma) \times \mathbb{R}$ be a weak solution of the scalar equation (2.29). Then $u \in \mathrm{H}^{1}(\Gamma)$ and $u$ is a weak solution of the elliptic equation

$$
-\Delta_{\Gamma} u+c u=g \quad \text { with } \quad c:=-\bar{a}_{\Gamma}, \quad g:=\bar{f}+K .
$$


Since $\Gamma$ is of class $\mathrm{C}^{k, \alpha}$ with $k \geq 2, \alpha \in(0,1)$, we have that

$$
c=-\bar{a}_{\Gamma}=8 \omega^{2}\left(x_{1} \nu_{1}+x_{2} \nu_{2}\right)+\nabla_{\Gamma} \nu_{\Gamma}: \nabla_{\Gamma} \nu_{\Gamma} \in \mathrm{C}^{k-2, \alpha}(\Gamma), \quad \bar{f}=f \cdot \nu_{\Gamma} \in \mathrm{C}^{k-2, \alpha}(\Gamma) .
$$

Furthermore, in a local chart there holds

$$
-\Delta_{\Gamma} u=-|g|^{1 / 2} D_{i}\left(g^{i j} \sqrt{|g|} D_{j} u\right)
$$

where $\left(g_{i j}\right) \in \mathbb{R}^{2 \times 2}$ are the components of the Riemannian metric of $\Gamma$ in the chosen coordinate system, $g^{i k} g_{k j}=\delta_{i j}$ and $|g|$ the determinant of $\left(g_{i j}\right)$. For a $\mathrm{C}^{k, \alpha}$-manifold we have $g_{i j} \in \mathrm{C}^{k-1, \alpha}(\Gamma)$.

It follows then by [AUBin 1982, Theorem 4.18] that $u \in \mathrm{C}^{k, \alpha}(\Gamma)$. We would like to mention at this point that Aubin's theorem requires that $c>0$ on $\Gamma$ but this is only needed to prove existence and uniqueness of a solution. The regularity of a solution is only affected by the regularity of the coefficients of the equation.

The important result is that a solution of the scalar linearised Euler-Lagrange equations is of the same differentiability class as $\Gamma$ itself.

For any weak solution $(w, K) \in W(\Gamma) \times \mathbb{R}$ of the vector-valued linearised Euler-Lagrange equations (2.30) we note that the term $a_{\Gamma} \nu_{\Gamma} \nu_{\Gamma} \cdot w$ - which couples the equations - is of class $\mathrm{C}^{k-2, \alpha}$ and can be added to the right-hand side. What remains is a Poisson-equation on a manifold which is covered by Aubin's theorem.

\subsubsection{TheOREM (EQUivalence of SCAlAR AND VECTOR-VALUED SOluTiOns)}

Let $\Gamma \subset \mathbb{R}^{3}$ be an isometrically immersed compact surface of class $\mathrm{C}^{k, \alpha}(k \geq 2$ and $0<\alpha<1)$ which possesses a reflective symmetry about the $\left(x_{1}, x_{2}\right)$-plane.

For $f \in\left(\mathrm{C}^{k-2, \alpha}(\Gamma)\right)^{3}$ and $b \in \mathbb{R}$ the following holds:

If each of the equations (2.29) and (2.30) of Definition 2.2.4 on the preceding page has a unique solution $(u, \tilde{K}) \in W(\Gamma) \times \mathbb{R}$ respectively $(w, K) \in W(\Gamma) \times \mathbb{R}$ then

$$
u \nu_{\Gamma} \equiv w \quad \text { and } \quad \tilde{K}=K
$$

Particularly, it holds that $v \nu_{\Gamma} \in \mathrm{C}^{k, \alpha}(\Gamma)$.

Proof: Assume that $(u, \tilde{K}) \in \underline{W}(\Gamma) \times \mathbb{R}$ and $(w, K) \in W(\Gamma) \times \mathbb{R}$ are the unique weak solutions of the scalar respectively vector-valued linearised Euler-Lagrange equations.

Then we first have $u^{\perp}=u \nu_{\Gamma} \in W(\Gamma)$ because $\nu_{\Gamma} \in \mathrm{C}^{k-1, \alpha}(\Gamma)$ and $k \geq 2$ and so $u^{\perp} \in \mathrm{H}^{1}(\Gamma)$.

By substituting $\left(u^{\perp}, \tilde{K}\right)$ and an arbitrary test-function $\chi \equiv\left\langle\chi, \nu_{\Gamma}\right\rangle \nu_{\Gamma} \equiv \bar{\chi} \nu_{\Gamma} \in W(\Gamma)$ 
into the right hand side of equation (2.30) it follows:

$$
\begin{aligned}
\int_{\Gamma} \nabla_{\Gamma} & \left(u \nu_{\Gamma}\right): \nabla_{\Gamma}\left(\bar{\chi} \nu_{\Gamma}\right) d o-\int_{\Gamma} a_{\Gamma} \nu_{\Gamma} \cdot\left(u \nu_{\Gamma}\right) \nu_{\Gamma} \cdot\left(\bar{\chi} \nu_{\Gamma}\right) d o-K \int_{\Gamma} \nu_{\Gamma} \cdot\left(\bar{\chi} \nu_{\Gamma}\right) d o \\
& =\int_{\Gamma} \nu_{\Gamma} \cdot \nu_{\Gamma} \nabla_{\Gamma} u \cdot \nabla_{\Gamma} \bar{\chi} d o+\int_{\Gamma}\left(\nabla_{\Gamma} \nu_{\Gamma}: \nabla_{\Gamma} \nu_{\Gamma}\right) u \chi d o-\int_{\Gamma} a_{\Gamma} u \bar{\chi} d o-\tilde{K} \int_{\Gamma} \bar{\chi} d o \\
& =\underbrace{\int_{\Gamma} \nabla_{\Gamma} u \cdot \nabla_{\Gamma} \bar{\chi} d o+\int_{\Gamma} \underbrace{\left(\nabla_{\Gamma} \nu_{\Gamma}: \nabla_{\Gamma} \nu_{\Gamma}-a_{\Gamma}\right)}_{=-\bar{a}_{\Gamma}} u \chi d o-\tilde{K} \int_{\Gamma} \bar{\chi} d o}_{\text {left-hand side of the scalar linearisation (2.29) }} \\
= & \int_{\Gamma} \bar{f} \bar{\chi} d o, \quad \text { because } \bar{\chi} \text { is an admissible scalar test-function } \\
= & \int_{\Gamma} f \cdot \nu_{\Gamma} \chi \cdot \nu_{\Gamma} d o .
\end{aligned}
$$

The term in the last line is the right hand side of the vector-valued linearisation (2.30). This already establishes that $\left(u \nu_{\Gamma}, \tilde{K}\right)$ is a weak solution of the vector-valued linearisation and hence - by the regularity result established in Theorem 2.2 .5 on page 35 - we have $u \nu_{\Gamma} \in \mathrm{C}^{k, \alpha}(\Gamma)$, despite the fact that $\nu_{\Gamma}$ is only of class $\mathrm{C}^{k-1, \alpha}$.

From the assumption of uniqueness we then get $\left(u \nu_{\Gamma}, \tilde{K}\right)=(w, K)$.

\subsection{The continuous Newton iteration}

We will now describe a Newton-type iteration to construct a new solution $\left(\Gamma_{m+1}, C_{m+1}\right)$ at an altered angular velocity $\omega_{m+1}:=\omega_{m}+\varepsilon_{\omega}$ from a known $\mathrm{C}^{\infty}$ solution $\left(\Gamma_{m}, C_{m}\right)$ at angular velocity $\omega_{m}($ with $m \in \mathbb{N})$.

To avoid confusion we like to comment on the kind of indices used throughout this section. The index " $m$ " in the statement above is used to indicate that $\Gamma_{m}$ and $\Gamma_{m+1}$ arise as two distinct surfaces during the path-tracking algorithm. Because $\Gamma_{m+1}$ is constructed by a Newton iteration we will need an additional index which denotes the sequence of surfaces constructed during that Newton iteration. For this we will use upper indices in brackets. An equivalent notation is used for the Lagrange-multipliers

For example, $\left(\Gamma_{m}^{(l)}, C_{m}^{(l)}\right)$ denotes the $l^{\text {th }}$ approximation constructed by the Newtonalgorithm from the $m^{\text {th }}$ exact solution $\left(\Gamma_{m}, C_{m}\right)$ in the course of the path-tracking algorithm. In particular we have

$$
\left(\Gamma_{m}, C_{m}\right)=\left(\Gamma_{m}^{(0)}, C_{m}^{(0)}\right) \quad \text { and } \quad\left(\Gamma_{m+1}, C_{m+1}\right):=\lim _{l \rightarrow \infty}\left(\Gamma_{m}^{(l)}, C_{m}^{(l)}\right)
$$

provided that the Newton method converges.

We are here in the semi-discrete regime, "discrete" because of the Newton iteration and because we track down solution paths in discrete steps with respect to the angular velocity $\omega$, but only semi-discrete because the Newton iteration described here will be established in terms of $\mathrm{C}^{k, \alpha}$ solutions of the linearised Euler-Lagrange equations (2.29) and (2.30) above, with $k \geq 2$ and $\alpha \in(0,1)$. 
This means that at this point of our analysis we discretise with respect to $\omega$. The discretisation "in space" - putting it in analogy to time-dependent problems - will be defined later by a finite element method.

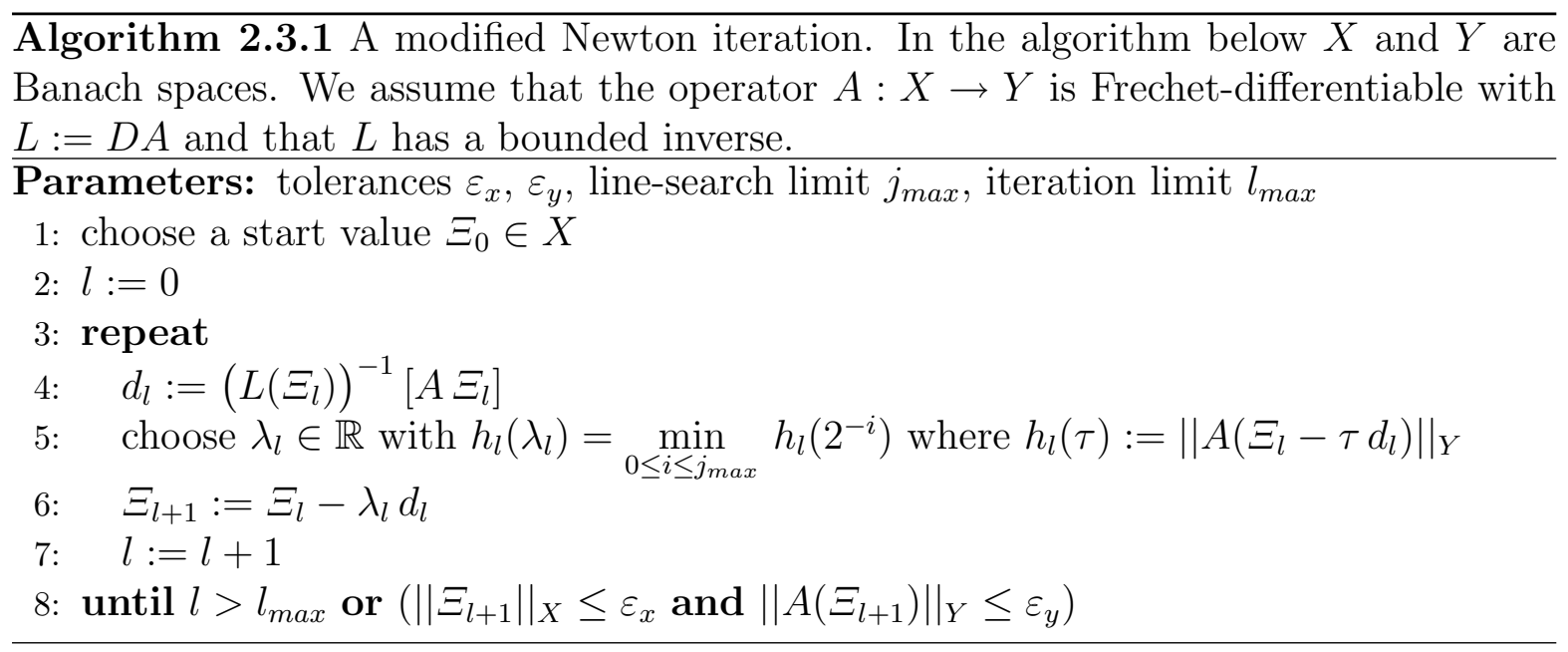

Algorithm 2.3.1 shows the general layout of the Newton iteration we are using.

We like to stress here that the entire discretisation is purely experimental. We do not even know whether the Newton-directions computed in each iteration step of the Newton-scheme exist, nor whether the operators defined in the following section are (Frechet-) differentiable.

A proof of convergence of the discretisation is entirely beyond the scope of this work.

In the following we assume that in the course of the path-tracking algorithm we have arrived at a "point" $\left(\Gamma_{m}, C_{m}\right)$. The goal is to develop a consistent notation which would define a Newton iteration if we knew that all quantities existed.

Because we are not concerned with convergence and hence will not perform the limiting process $\left(\Gamma_{m+1}, C_{m+1}\right)=\lim _{l \rightarrow \infty}\left(\Gamma_{m}^{(l)}, C_{m}^{(l)}\right)$ we drop the lower index and set $(\Gamma, C):=\left(\Gamma_{m}, C_{m}\right)$ in the following. For the same reason we set $\omega:=\omega_{m+1}$.

\subsubsection{The differential operators}

Let $\Xi_{0} \equiv\left(\Gamma^{(0)}, C^{(0)}\right):=(\Gamma, C)$ where $\Gamma \subset \mathbb{R}^{3}$ is a compact immersed surface of class $\mathrm{C}^{\infty}$.

Because the curvature of $\Gamma$ is bounded there is an open set $U_{0}^{k, \alpha} \subset\left(\mathrm{C}^{k, \alpha}(\Gamma)\right)^{3}$ so that each $\varphi \in U_{0}^{\infty}:=U_{0}^{k, \alpha} \cap\left(\mathrm{C}^{\infty}(\Gamma)\right)^{3}$ defines a diffeomorphism $\operatorname{id}_{\Gamma}+\varphi: \Gamma \rightarrow \Gamma_{\varphi}$ with

$$
\Gamma_{\varphi}:=\left\{\operatorname{id}_{\Gamma}(X)+\varphi(X) \mid X \in \Gamma\right\}
$$

where $\Gamma_{\varphi}$ is again of class $\mathrm{C}^{\infty}$.

We remark that the Banach spaces $\mathrm{C}^{j, \beta}\left(\Gamma_{\varphi}\right)$ and $\mathrm{C}^{j, \beta}(\Gamma)$ are norm-equivalent for $j \leq k$ and $\beta \leq \alpha$ and that the norm-equivalence between them is controlled by the $\mathrm{C}^{j, \beta}(\Gamma)$ norm of $\operatorname{id}_{\Gamma}+\varphi$. Similarly, the Sobolev spaces $\mathrm{H}_{p}^{s}\left(\Gamma_{\varphi}\right)$ and $\mathrm{H}_{p}^{s}(\Gamma), s<j+\beta$, are 
equivalent where the equivalence is controlled by the $\mathrm{H}_{p}^{s}(\Gamma)$ norm of $\operatorname{id}_{\Gamma}+\varphi$ (chain rule) and the $\mathrm{H}_{\infty}^{1}(\Gamma)$ norm of $\mathrm{id}_{\Gamma}+\varphi$ (transformation formula for integrals).

By actually identifying the spaces $\mathrm{C}^{j, \beta}\left(\Gamma_{\varphi}\right)$ and $\mathrm{C}^{j, \beta}(\Gamma)$ we can define an operator

$$
A^{\infty}: U_{0}^{\infty} \times \mathbb{R} \rightarrow \mathrm{C}^{k-2, \alpha}(\Gamma) \times \mathbb{R}:(\varphi, K) \mapsto\left(R_{\left(\Gamma_{\varphi}, K, \omega\right)}, \int_{\Gamma_{\varphi}} \operatorname{id}_{\Gamma_{\varphi}} \cdot \nu_{\Gamma_{\varphi}} d o-4 \pi\right)
$$

where

$$
R_{\left(\Gamma_{\varphi}, K, \omega\right)}:=2 H_{\Gamma_{\varphi}}-\left(4 \omega^{2} r^{2}+\right) K=-\nu_{\Gamma_{\varphi}} \cdot \Delta_{\Gamma_{\varphi}} \mathrm{id}_{\Gamma_{\varphi}}-\left(4 \omega^{2} r^{2}+K\right)
$$

is the residual of the non-linear Euler-Lagrange equations (2.6) on page 23. This notation was already introduced when discussing the second variations (2.21) and (2.22) of the normalised energy $E$ on page 28.

The operator $A$ is continuous on $U_{0}^{\infty} \times \mathbb{R}$ and $U_{0}^{\infty}$ is dense in $U_{0}^{k, \alpha}$ so that we can extend $A$ to a continuous operator on $U_{0}^{k, \alpha} \times \mathbb{R}$. We note that $A$ is Gateaux-differentiable on $U_{0}^{\infty} \times \mathbb{R}$ and we assume in the following that $A$ is indeed Frechet-differentiable on $U_{0}^{k, \alpha} \times \mathbb{R}$.

To formulate the iteration step of the Newton iteration we need an expression for $D A(\varphi, K)$ only at points $(\varphi, K) \in U_{0}^{\infty} \times \mathbb{R}$ where $D A$ denotes the Frechet-derivative of the operator $A$. The expression for $D A(\varphi, K)$ is implied by the vector-valued linearised Euler-Lagrange equations (2.23) on page 28:

$$
\begin{aligned}
& D A(\varphi, K):\left(\mathrm{C}^{k, \alpha}\left(\Gamma_{\varphi}\right)\right)^{3} \times \mathbb{R} \rightarrow \mathrm{C}^{k-2, \alpha}\left(\Gamma_{\varphi}\right) \times \mathbb{R}, \\
& (\psi, J) \mapsto\left(-\nu_{\Gamma_{\varphi}} \cdot \Delta_{\Gamma_{\varphi}} \psi-a_{\Gamma_{\varphi}} \nu_{\Gamma_{\varphi}} \nu_{\Gamma_{\varphi}} \cdot \psi-L+R_{\left(\Gamma_{\varphi}, K, \omega\right)} Y_{\Gamma_{\varphi}} \cdot \psi, \int_{\Gamma_{\varphi}} \psi \cdot \nu_{\Gamma_{\varphi}} d o\right) .
\end{aligned}
$$

The $0^{\text {th }}$-order term $a_{\Gamma_{\varphi}}$ was defined by equation $(2.25)$ on page 28 and is governed by the square of the second fundamental form of $\Gamma_{\varphi}$.

The expression $\left(R_{\left(\Gamma_{\varphi}, K, \omega\right)} Y_{\Gamma_{\varphi}} \cdot \psi\right)$ is the correction introduced by the fact that we necessarily linearise at a non-stationary point $\left(\Gamma_{\varphi}, K\right)$ of the drop-energy, compare with equation (2.21) on page 27.

We can make $R_{\Gamma_{\varphi}, K, \omega}$ arbitrarily small by choosing the start value $\Gamma$ closer to a solution of the Euler-Lagrange equation (2.6). In the Newton iteration Algorithm 2.3.1 on the facing page we will therefore use the following approximation

$$
L(\varphi, K)[\psi, J]:=\left(-\nu_{\Gamma_{\varphi}} \cdot \Delta_{\Gamma_{\varphi}} \psi-a_{\Gamma_{\varphi}} \psi \cdot \nu_{\Gamma_{\varphi}}-L, \int_{\Gamma_{\varphi}} \psi \cdot \nu_{\Gamma_{\varphi}} d o\right) .
$$

instead of $D A(\varphi, K) \approx L(\varphi, K)$.

As we have seen in the discussion of the linearised Euler-Lagrange equations the operator $A$ and its approximate linearisation $L$ are not invertible in general. The reason is that $A$ - in the way $A$ is defined above - has a non-trivial Lie-symmetry group.

We will resolve this problem by constructing the Newton-correction in special Ansatzspaces, see Remark 2.2.3 on page 34 . 


\subsubsection{The iteration step}

Using the notation of the preceding section we are now able to constitute the iteration step of the Newton algorithm Algorithm 2.3.1 on page 38 in our special case.

Let $\Xi_{l}=\left(\operatorname{id}_{\Gamma^{(l)}}, C^{(l)}\right) \in\left(\mathrm{C}^{k, \alpha}\left(\Gamma^{(0)}\right)\right)^{3} \times \mathbb{R}$ be the point generated in the previous iteration step, where $\Gamma^{(l)}$ is the associated surface. We then generate $\Gamma^{(l+1)}$ and thereby $\Xi_{l+1}=\left(\mathrm{id}_{\Gamma^{(l+1)}}, C^{(l+1)}\right)$ by the following (sub-) steps:

\section{Algorithm 2.3.2 The iteration step of the continuous Newton iteration}

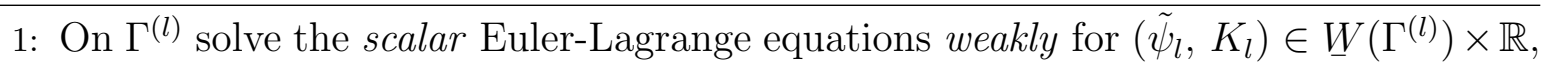
as defined in Definition 2.2.4 on page 35:

$$
\int_{\Gamma^{(l)}} \nabla_{\Gamma^{(l)}} \tilde{\psi}_{l} \cdot \nabla_{\Gamma^{(l)}} \eta d o-\int_{\Gamma^{(l)}} \bar{a}_{\Gamma^{(l)}} \tilde{\psi}_{l} \eta d o=\int_{\Gamma^{(l)}}\left(\bar{f}+\bar{a}_{\Gamma^{(l)}} \frac{b}{\left|\Gamma^{(l)}\right|}+K_{l}\right) \eta d o
$$

for all $\eta \in \underline{W}\left(\Gamma^{(l)}\right)$ with "right-hand side"

$$
(f, b):=A\left(\Xi_{l}\right)=\left(R_{\left(\Gamma^{(l)}, C^{(l)}, \omega\right)}, \int_{\Gamma^{(l)}} \operatorname{id}_{\Gamma^{(l)}} \cdot \nu_{\Gamma^{(l)}} d o\right)
$$

and let $\bar{\psi}_{l}:=\tilde{\psi}_{l}+\frac{b}{\left|\Gamma^{(l)}\right|}$. The special choice of the Ansatz space $\underline{W}\left(\Gamma^{(l)}\right) \subset \mathrm{H}^{1}\left(\Gamma^{(l)}\right)$ eliminates the non-uniqueness caused by the Lie-symmetries as explained in Section 2.2 above.

2: Set $\psi_{l}:=\bar{\psi}_{l} \nu_{\Gamma^{(l)}}$. By the regularity Theorem 2.2.5 on page 35 and the equivalence Theorem 2.2.6 on page 36 we have $\psi_{l} \in \mathrm{C}^{k, \alpha}\left(\Gamma^{(l)}\right)$ and $\left(\psi_{l}, K_{l}\right)$ is the solution of the vector-valued linearised Euler-Lagrange equation (2.26). This implies

$$
d_{l}:=\left(\psi_{l}, K_{l}\right)=\left(L\left(\operatorname{id}_{\Gamma^{(l)}}, C^{(l)}\right)\right)^{-1} A\left(\operatorname{id}_{\Gamma^{(l)}}, C^{(l)}\right) .
$$

3: Choose $\lambda_{l} \in \mathbb{R}^{+}$as explained in Algorithm 2.3.1 on page 38 and generate

$$
\Xi_{l+1}=\left(\operatorname{id}_{\Gamma^{(l+1)}}, C^{(l+1)}\right):=\left(\operatorname{id}_{\Gamma^{(l)}}, C^{(l)}\right)-\lambda_{l}\left(\psi_{l}, K_{l}\right)=\Xi_{l}-\lambda_{l} d_{l},
$$

where $\Gamma^{(l+1)}:=\left\{\operatorname{id}_{\Gamma^{(l)}}(X)-\lambda_{l} \psi_{l}(X) \mid X \in \Gamma^{(l)}\right\}=\Gamma^{(l)}{ }_{-\lambda_{l}} \psi_{l}$, see Figure 2.3.

REmARK 2.3.1 1. The Newton iteration produces a sequence of surfaces and associated function spaces, which are generated by the diffeomorphisms

$$
\Psi_{l}:=\mathrm{id}_{\Gamma^{(l)}}-\lambda_{l} \psi_{l} \in \mathrm{C}^{k, \alpha}\left(\Gamma^{(l)}\right), \quad l \in \mathbb{N}_{0} .
$$

In the definition of the operator $A$ and its approximate linearisation $L$ we have identified all those function spaces with the corresponding spaces over $\Gamma^{(0)}$. The identification is arranged by chaining up all diffeomorphisms $\Psi_{0}, \ldots, \Psi_{j-1}$ which lead to a surface $\Gamma^{(j)}$. The relation between the function spaces is demonstrated by the following diagram for the case of $\mathrm{C}^{k, \alpha}$ :

$$
\begin{array}{cccccc}
\Gamma^{(0)} & \stackrel{\Psi_{0}}{\longrightarrow} \quad \Gamma^{(1)} \stackrel{\Psi_{1}}{\longrightarrow} \cdots \stackrel{\Psi_{l-1}}{\longrightarrow} \Gamma^{(l)} \stackrel{\Psi_{l}}{\longrightarrow} \cdots \\
\mathrm{C}^{k, \alpha}\left(\Gamma^{(0)}\right) & \stackrel{\Psi_{0 *}}{\longleftarrow} \mathrm{C}^{k, \alpha}\left(\Gamma^{(1)}\right) \stackrel{\Psi_{1 *}}{\longleftarrow} \cdots \stackrel{\Psi_{l-1^{*}}}{\longleftarrow} \mathrm{C}^{k, \alpha}\left(\Gamma^{(l)}\right) \stackrel{\Psi_{l^{*}}}{\longleftarrow} \cdots
\end{array}
$$


Figure 2.3 Displacement in normal direction during the Newton iteration

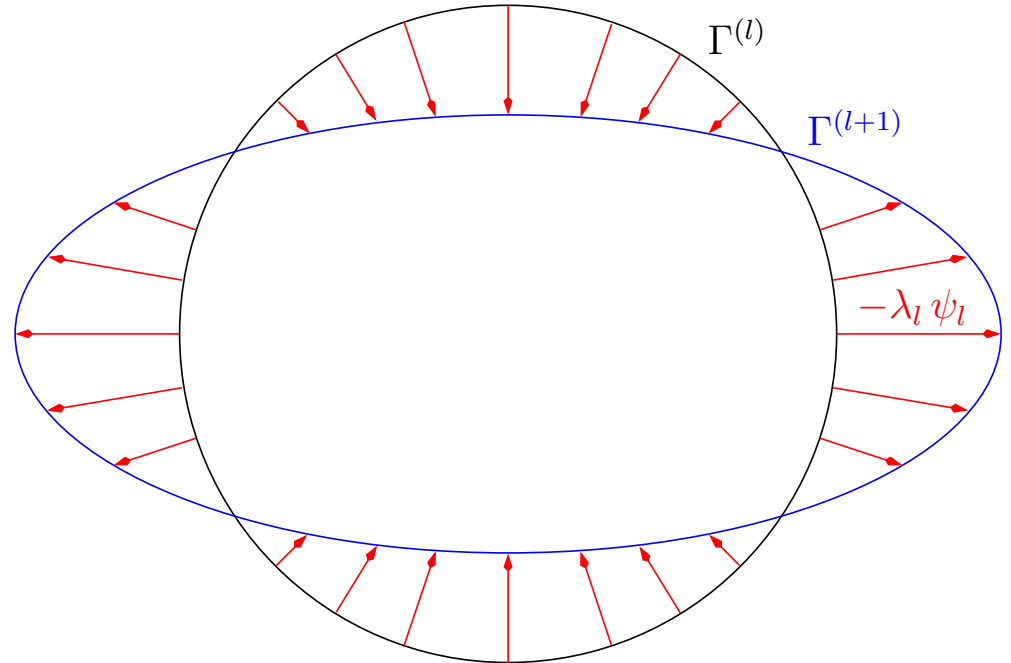

Here $\Phi * f(x)=f(\Phi(x))$ denotes the pull-back which maps a space $\mathrm{C}^{k, \alpha}\left(\Gamma^{(l)}\right)$ back to $\mathrm{C}^{k, \alpha}\left(\Gamma^{(l-1)}\right)$. The equivalence between $\mathrm{C}^{k, \alpha}\left(\Gamma^{(l)}\right), l \in \mathbb{N}$, and the "base"space $\mathrm{C}^{k, \alpha}\left(\Gamma^{(0)}\right)$ is controlled by the $\mathrm{C}^{k, \alpha}$-norm of the chain of diffeomorphisms $\Psi_{l-1} \circ \cdots \circ \Psi_{0}$.

2. We have formulated the iteration step of the Newton algorithm in terms of $\mathrm{C}^{k, \alpha_{-}}$ spaces to indicate in which norm convergence possibly could take place. This should probably be weakened. However, choosing for example a $\mathrm{H}_{p}^{k}$-norm one would still need to have $\mathrm{H}_{\infty}^{1}$-estimates for the diffeomorphisms $\Psi_{j}, j \in \mathbb{N}$, to control the equivalence of the function spaces over the sequence of surfaces generated by the Newton iteration.

3. Requiring the initial surface to be of differentiability class $\mathrm{C}^{\infty}$ is not a restriction. The initial surfaces for the path-tracking algorithm are chosen from the exactly known families of surfaces which are indeed $\mathrm{C}^{\infty}$-manifolds.

4. To actually establish convergence of the Newton iteration in any appropriate norm one would need to have a priori estimates for the solutions of the linearised EulerLagrange equations. To establish such estimates one needs control over the $0^{\text {th }}$ order term $-a_{\Gamma}$ respectively $-\bar{a}_{\Gamma}$. However, $a_{\Gamma}$ is governed by $\nabla_{\Gamma} \nu_{\Gamma}: \nabla_{\Gamma} \nu_{\Gamma}$, the square of the second fundamental form. Already in the course of the annular family established by [GULLIVER 1984] the term $\nabla_{\Gamma} \nu_{\Gamma}: \nabla_{\Gamma} \nu_{\Gamma}$ diverges when the annular shapes approach the spheroidal family at $\omega^{2}=1 / 2$, see Section 1.4.

5. It is important that control over the regularity of the surface is not destroyed during the Newton-algorithm. While the smoothness of the initial manifold guarantees that each surface $\Gamma^{(l)}$ is again smooth, it is the equivalence Theorem 2.2.6 on page 36 which guarantees that we do not loose regularity by computing only the normal component of the Newton-directions $\psi_{l}$. Here, "loss of regularity" means loss of information about the norm of the derivatives of $\psi_{l}$ in the given function space. 


\subsection{The discretised Newton iteration}

The Euler-Lagrange equations derived in Section 2.1 which constitute the Newton iteration described in the previous section are defined on the surface of the drop. We do not want to use a global parametrisation to avoid coordinate singularities. Using, for example, a polar coordinate system one even could not represent the exactly known solution families in that coordinate system (see Figure 2.4).

$\overline{\text { Figure 2.4 A parametrisation by a polar coordinate system fails even along the family }}$ of exact spheroidal solutions found by [BEER 1869]. The red spots mark the points where the radius vector intersects the surface.

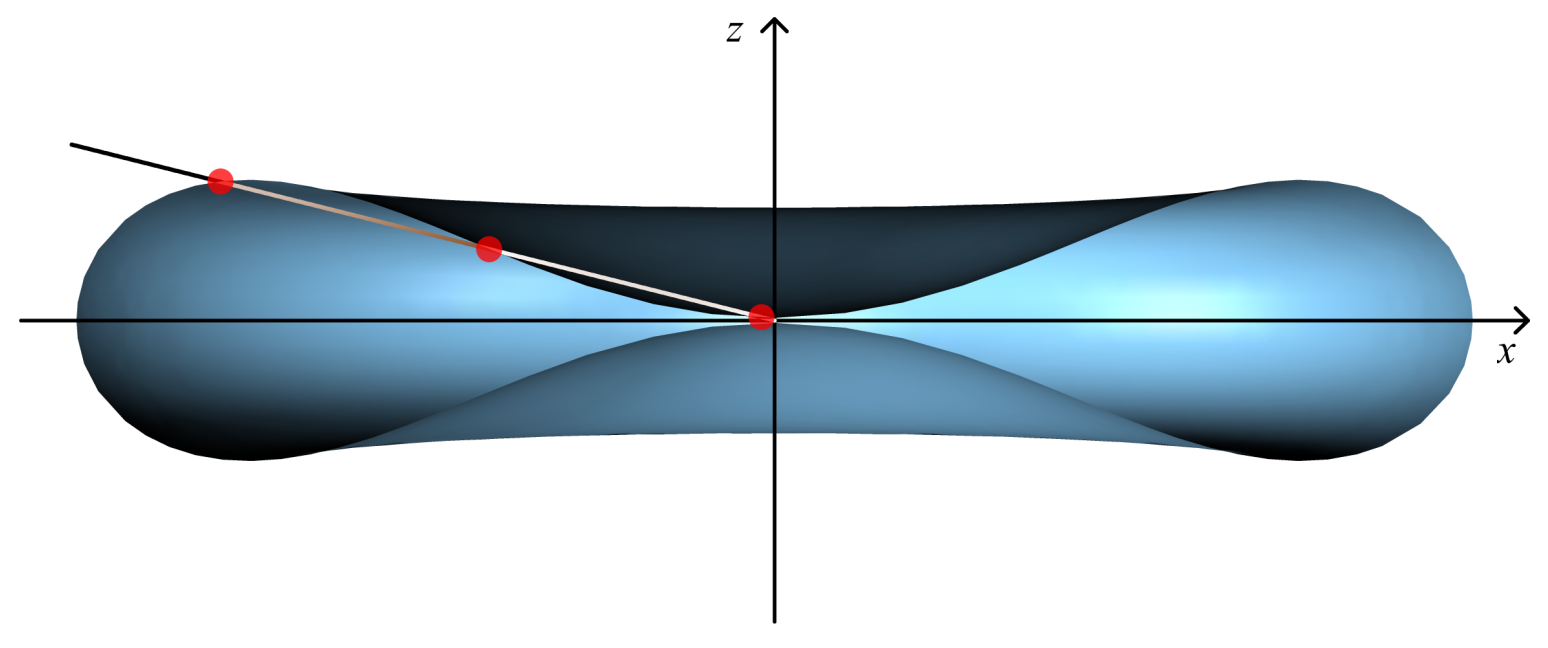

Therefore we follow the method developed in [DZIUK 1991] for parametric meancurvature flow and adapt it to our problem. In this approach one defines finite element spaces on discretised surfaces and solves the differential equations on the surfaces without using an explicit parametrisation in the sense that the local parametrisations are defined by the triangulation itself. The construction of these parametric finite elements is described below in Section 2.4.1.

In the following sections we use these parametric finite elements to discretise the continuous Newton iteration defined in the preceding Section 2.3 and to discretise the eigenvalue problem originating from the bifurcation equations in the sense of Theorem 1.3.28 on page 15 .

Finally we will give an overview of the complete discrete path-tracking algorithm used in this work.

\subsubsection{Iso-parametric triangulations}

First we need to construct a discretisation $\Gamma_{h}$ of a smooth surface $\Gamma$ to have an initial value for the discretisation of the path-tracking algorithm. The notion "iso-parametric triangulation" will be justified in the following Section 2.4.2.

We start by giving the necessary notations: 


\subsubsection{Definition (Regular Simplex)}

Let $P_{1}, \ldots, P_{n+1} \in \mathbb{R}^{q}$, with $n, q \in \mathbb{N}, n \leq q$, such that $P_{1}-P_{n+1}, \ldots, P_{n}-P_{n+1}$ are linearly independent. The regular $n$-simplex $T$ with vertices $P_{1}, \ldots, P_{n+1}$ is the convex hull of the points $P_{1}, \ldots, \overline{P_{n+1} \text { : }}$

$$
T:=\operatorname{conv}\left(P_{1}, \ldots, P_{n+1}\right)=\left\{X \in \mathbb{R}^{q} \mid X=\sum_{i=1}^{n+1} \lambda_{i} P_{i}, \lambda_{i} \in \mathbb{R}^{\geq 0}, \sum_{i=1}^{n+1} \lambda_{i}=1\right\}
$$

For $X \in T$ the components of the $(n+1)$-tuple $\left(\lambda_{1}, \ldots, \lambda_{n+1}\right)$ are called the barycentric coordinates of $X$ with respect to $T$.

For $k \leq n$ a regular $k$-simplex with vertices $Q_{1}, \ldots, Q_{k+1} \in\left\{P_{1}, \ldots, P_{n+1}\right\}$ is called a $k$-sub-simplex of $T$. Any $(n-1)$-sub-simplex of $T$ is called a face of $T$. Any 1-subsimplex of $T$ is called an edge of $T$.

REMARK 2.4.1 We introduce the following notation:

The diameter of $T$ and the radius of the largest $n$-dimensional ball contained in $T$ are denoted by

$$
h(T):=\operatorname{diam}(T) \quad \text { and } \quad \rho(T):=\max \left\{r \in \mathbb{R}^{+} \mid B_{r}^{T}(X) \subset T, X \in T\right\},
$$

where $B_{r}^{T}(X)$ denotes the $n$-dimensional section of the $q$-dimensional ball with radius $r$ around $X \in \mathbb{R}^{q}$ with the affine sub-space of dimension $n$ containing $T$.

2.4.2 Definition (Regular Triangulation, Conforming Triangulation) Let $q, n \in \mathbb{R}, n \leq q$ and $N \in \mathbb{N}$. Let $T_{j} \subset \mathbb{R}^{q}, 1 \leq j \leq N$, be regular $n$-simplices and let

$$
h:=\max _{1 \leq j \leq N} h\left(T_{j}\right), \quad \overline{\Gamma_{h}}:=\bigcup_{j=1}^{N} T_{j} \quad \text { and } \quad \mathcal{T}_{h}:=\bigcup_{j=1}^{N}\left\{T_{j}\right\}
$$

1. $\mathcal{T}_{h}$ is called a conforming triangulation if for any $T_{j}, T_{l} \in \mathcal{T}_{h}$ with $T_{j} \neq T_{l}$ the

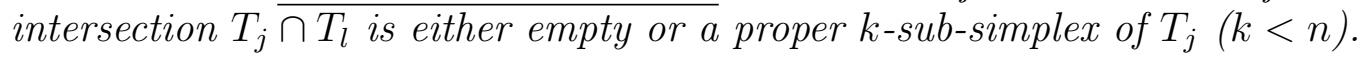

2. A vertex $P$ of a regular $n$-simplex $T_{j} \in \mathcal{T}_{h}$ is called a non-conforming vertex of $\mathcal{T}_{h}$ if there is a regular $n$-simplex $T_{l} \in \mathcal{T}_{h}$ such that $P$ is not a vertex of $T_{l}$, but $P \in T_{l}$.

3. A sequence $\left(\mathcal{T}_{h_{j}}\right)_{j \in \mathbb{N}}$ of triangulations is called shape-regular if

$$
\sup _{j \in \mathbb{N}} \max _{T \in \mathcal{T}_{h_{j}}} \frac{h(T)}{\rho(T)}<\infty
$$

REMARK 2.4.2 We note that for any regular triangulation $\mathcal{T}_{h}$ of regular $n$-simplices its associated simplicial domain $\Gamma_{h} \subset \mathbb{R}^{q}$ is an $n$-dimensional $\mathrm{C}^{0,1}$-immersion into $\mathbb{R}^{q}$. For a smooth $n$-dimensional immersion $\Gamma$ we say that $\Gamma_{h}$ and $\mathcal{T}_{h}$ interpolate $\Gamma$ if for any vertex $P \in \mathcal{T}_{h}$ we have $P \in \Gamma$. 
In our case the above definitions are only needed for the case of conforming triangulations consisting of regular 2-simplices in $\mathbb{R}^{3}$.

To get a discretisation of an initial surface for the path-tracking algorithm the goal is now to construct a $\mathrm{C}^{0,1}$-immersion $\Gamma_{h}^{d}$ which is locally parametrised by polynomials of a given degree $d$ and which approximates a smooth surface $\Gamma$. To this aim we start with a conforming triangulation $\mathcal{T}_{h}$ which interpolates $\Gamma$.

It is possible to construct such a triangulation from local charts (see [KALIK and WendLAND 1992]), but this is not necessary in our case. Our initial surface will either be a spheroid close to the unit-sphere or an annular surface close to a circular torus. In both cases we have a global parametrisation and can choose an initial "macro"-triangulation which interpolates the smooth surface. Later, this macro-triangulation is refined sufficiently often to yield a good approximation of $\Gamma$. The details of the refinement process and how to maintain regularity of the triangulation is described in the following chapter in the sections 3.3 and 3.4.

Therefore, at this point we just assume that we already have a conforming triangulation $\mathcal{T}_{h}^{1}:=\mathcal{T}_{h}$ which interpolates a smooth surface $\Gamma$.

If the desired order of the polynomial parametrisation is 1 then we are done. Otherwise we use Lagrange-polynomials of order $d$ over a reference-simplex to define higher-order approximations of $\Gamma$ by the procedure described below. We define the following notations:

\subsubsection{Definition (Reference Simplex, Lagrange Polynomials)}

The regular $n$-simplex $\hat{T} \subset \mathbb{R}^{n+1}$ defined by

$$
\hat{T}:=\left\{\left(\lambda_{1}, \ldots, \lambda_{n+1}\right) \mid \lambda_{i} \in \mathbb{R}^{\geq 0}, \sum_{i=1}^{n+1} \lambda_{i}=1\right\}
$$

is called the $n$-dimensional reference simplex.

For given $d \in \mathbb{N}$ we denote by $m(n, d)$ the dimension of the vector space $P_{d}(\hat{T})$ of polynomials of degree $d$ over $\hat{T} \subset \mathbb{R}^{n+1}$. The canonical Lagrange-interpolation nodes on $\hat{T}$ are denoted by $\hat{Q}_{j}$ and the nodal basis functions by $\hat{p}_{j}, j=1, \ldots, m(n, d)$.

REMark 2.4.3 Per definition we have $\hat{p}_{j}\left(\hat{Q}_{i}\right)=\delta_{i j}$. Examples for $n=2$ and $d=1,2$ are shown in Figure 2.5(b) on the facing page.

We give now the construction of a $\mathrm{C}^{0,1}$ surface $\Gamma_{h}^{d}$ which is locally parametrised over the reference simplex $\hat{T}$ by polynomials of degree $d$ and interpolates the smooth surface $\Gamma$. An example is illustrated in Figure 2.5(a) on the next page.

We still assume that we have a given regular triangulation $\mathcal{T}_{h}^{1}$ which interpolates the smooth surface $\Gamma$ and also a well defined projection $\Pi_{\Gamma}: \Gamma_{h}^{1} \rightarrow \Gamma: P \mapsto \Pi_{\Gamma}(P) \in \Gamma$. In general $\Pi_{\Gamma}$ exists only if the triangulation $\mathcal{T}_{h}^{1}$ is fine enough.

For any regular $n$-simplex $\bar{T} \in \mathcal{T}_{h}^{1}$ with vertices $Q_{1}, \ldots, Q_{n+1} \in \mathbb{R}^{q}$ we denote by $\Phi_{T}^{1}: \hat{T} \rightarrow T$ the affine linear parametrisation which maps each vertex $\hat{Q}_{i}$ of $\hat{T}$ to the vertex $Q_{i}$ of $\bar{T}$, so we have

$$
\Phi_{T}^{1}\left(\hat{Q}_{i}\right)=Q_{i}, \quad 1 \leq i \leq(n+1) .
$$




\section{Figure 2.5 Parametrisation with Lagrange finite elements}

(a) The figure shows an octahedron as "macro"-triangulation and the resulting interpolation of the unit-sphere with quadratic Lagrange elements. The images $Q_{i}$ of the Lagrange nodes $\hat{Q}_{i}$ of the reference element $\hat{T}$ are obtained by first mapping $\hat{T}$ with an affine-linear transformation to each face of the octahedron. Then the non-vertex Lagrange nodes of the affine-linear image are projected to the sphere. $\Phi_{T}^{2}$ is the resulting local parametrisation which interpolates the sphere at the points $Q_{i}$.

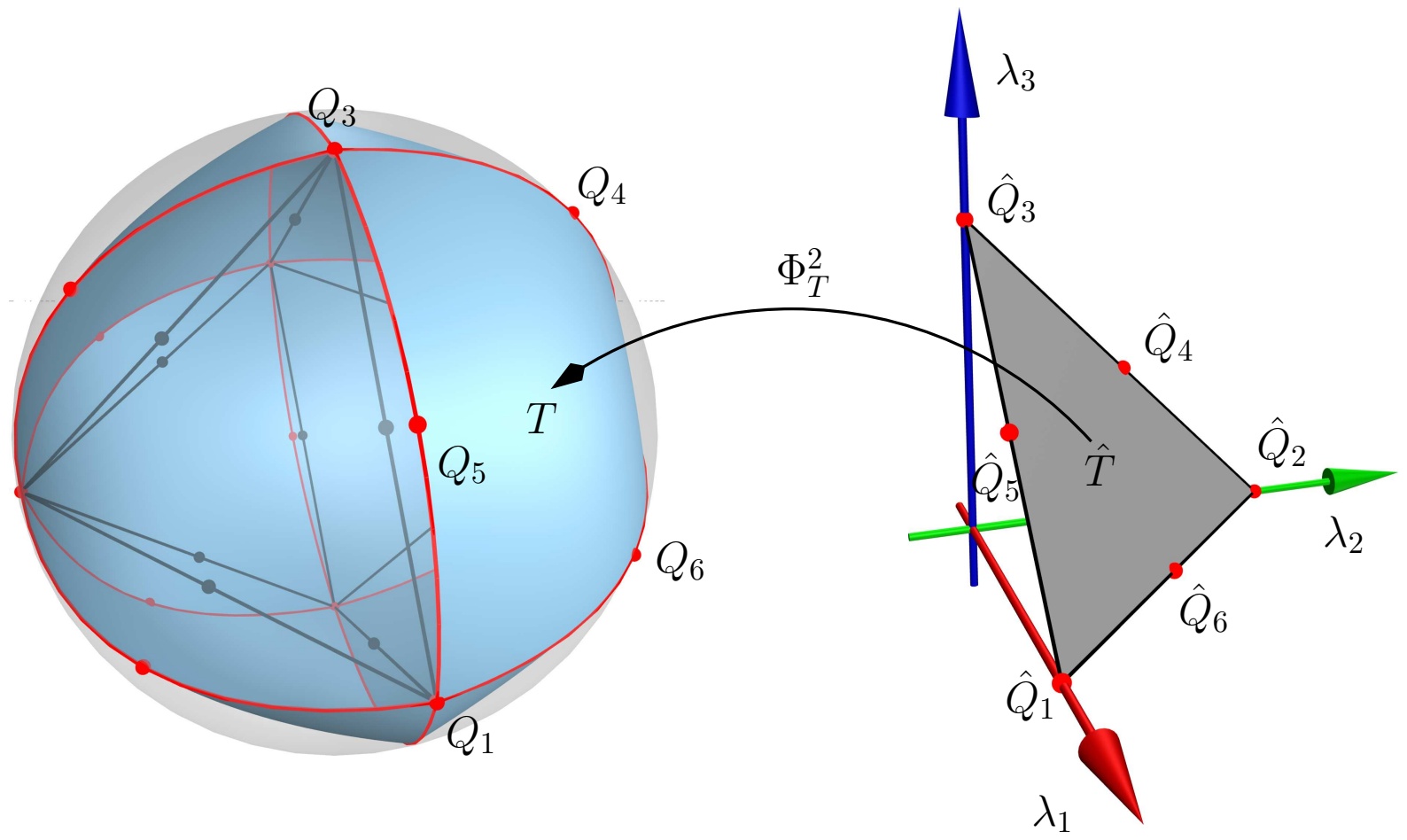

(b) Lagrange nodes and basis-functions for linear and quadratic finite elements. $n$ denotes the dimension of the reference element $\hat{T} \subset \mathbb{R}^{n+1} . d$ is the polynomial degree of the Ansatz-functions and $m(n, d)$ is the number of Lagrange nodes, which is also the number of the Lagrange Ansatz-functions for given $n$ and $d$.

$n=2, d=1$

$$
\begin{aligned}
& m(n, d)=3, \\
& \hat{Q}_{1}=(1,0,0), \quad \hat{Q}_{2}=(0,1,0), \quad \hat{Q}_{3}=(0,0,1), \\
& \hat{p}_{j}\left(\lambda_{1}, \lambda_{2}, \lambda_{3}\right)=\lambda_{j}
\end{aligned}
$$

$n=2, d=2$

$$
\begin{aligned}
& m(n, d)=6, \\
& \hat{Q}_{1}=(1,0,0), \quad \hat{Q}_{2}=(0,1,0), \quad \hat{Q}_{3}=(0,0,1), \\
& \hat{Q}_{4}=\left(0, \frac{1}{2}, \frac{1}{2}\right), \quad \hat{Q}_{5}=\left(\frac{1}{2}, 0, \frac{1}{2}\right), \quad \hat{Q}_{6}=\left(\frac{1}{2}, \frac{1}{2}, 0\right), \\
& \hat{p}_{j}\left(\lambda_{1}, \lambda_{2}, \lambda_{3}\right)= \begin{cases}2 \lambda_{j}\left(\lambda_{j}-1\right) & j=1,2,3, \\
4 \lambda_{2} \lambda_{3} & j=4, \\
4 \lambda_{3} \lambda_{1} & j=5, \\
4 \lambda_{1} \lambda_{2} & j=6 .\end{cases}
\end{aligned}
$$


By $\bar{Q}_{i}:=\Phi_{T}^{1}\left(\hat{Q}_{i}\right),(n+1)<i \leq m(n, d)$, we denote the images of the non-vertex Lagrange-nodes of $\hat{T}$ on the simplex $\bar{T}$ and set

$$
Q_{i}:=\Pi_{\Gamma}\left(\bar{Q}_{i}\right)=\Pi_{\Gamma} \circ \Phi_{T}^{1}\left(\hat{Q}_{i}\right), \quad(n+1)<i \leq m(n, d) .
$$

This defines a polynomial mapping $\Phi_{T}^{d}$ by interpolating the points $Q_{i} \in \Gamma$ with the Lagrange-polynomials $\hat{p}_{i}, 1 \leq i \leq m(n, d)$,

$$
\Phi_{T}^{d}: \hat{T} \rightarrow T:=\Phi_{T}^{d}(\hat{T}): \lambda=\left(\lambda_{1}, \ldots, \lambda_{n+1}\right) \mapsto \sum_{i=1}^{m(n, d)} \hat{p}_{i}(\lambda) Q_{i} .
$$

REMARK 2.4.4 By construction $T$ interpolates the surface $\Gamma$ in its Lagrange-nodes $Q_{i}=\Phi_{T}^{d}\left(\hat{Q}_{i}\right)$. If the base-triangulation $\mathcal{T}_{h}^{1}$ is fine enough, then $\Phi_{T}^{d}: \hat{T} \rightarrow T$ is a diffeomorphism. If $\Phi_{T}^{d}$ is a diffeomorphism for all $\bar{T} \in \mathcal{T}_{h}^{1}$ then

$$
\Gamma_{h}^{d}:=\bigcup_{\bar{T} \in \mathcal{T}_{h}^{1}} \sum_{i=1}^{m(n, d)} \hat{p}_{i}(\hat{T})\left(\Pi_{\Gamma} \circ \Phi_{\bar{T}}^{1}\left(\hat{Q}_{i}\right)\right)
$$

is a $\mathrm{C}^{0,1}$-surface which is piecewise polynomial of degree $d$ over the reference element $\hat{T}$. The continuity of $\Gamma_{h}^{d}$ is clear, because the parametrisation with Lagrange-polynomials over the reference elements respects the simplicial hierarchy of $\mathcal{T}_{h}^{1}$. Therefore neighbouring elements have compatible Lagrange-nodes in their intersection (see the example in Figure 2.5(a) on the preceding page).

In the following we will also need $\mathrm{C}^{0,1}$-surfaces which do not originate from the interpolation of an underlying smooth surface $\Gamma$. For these surfaces we can generalise our definitions in the following sense:

\subsubsection{DeFinition (ISO-PARAMETRIC $n$-SimpleX)}

Let $Q_{i} \in \mathbb{R}^{q}, 1 \leq i \leq m(n, d)$, such that the mapping

$$
\Phi_{T}^{d}: \hat{T} \rightarrow T:=\Phi_{T}^{d}(\hat{T}): \lambda=\left(\lambda_{1}, \ldots, \lambda_{n+1}\right) \mapsto \sum_{i=1}^{m(n, d)} \hat{p}_{i}(\lambda) Q_{i}
$$

is a diffeomorphism. Then $T$ is called an iso-parametric $n$-simplex with vertices $Q_{1}, \ldots, Q_{n+1}$ and non-vertex nodes $Q_{n+2}, \ldots, \overline{Q_{m(n, d)}}$.

REMARK 2.4.5 Likewise it is possible to define conforming iso-parametric "triangulations" $\mathcal{T}_{h}^{d}$ as sets of compatible iso-parametric $n$-simplices in the sense that intersecting iso-parametric $n$-simplices have compatible Lagrange-nodes in their intersection.

In this sense $\mathcal{T}_{h}^{d}$ is in the following such a conforming iso-parametric triangulation which does not necessarily arise from the interpolation of a smooth surface. Its associated $\mathrm{C}^{0,1}$-immersion will be denoted by $\Gamma_{h}^{d}:=\bigcup_{T \in \mathcal{T}_{h}^{d}} T$. Because for any degree $d$ the first $(n+1)$ Lagrange-nodes of the reference-element $\hat{T}$ are its vertices, we have always a canonical linear triangulation $\mathcal{T}_{h}^{1}$ and an associated immersion $\Gamma_{h}^{1}$ which interpolate the discrete surface $\Gamma_{h}^{d}$.

We will omit the upper index which indicates the polynomial degree of the local parametrisation over the reference element $\hat{T}$ when it is either clear from the context or does not matter. 


\subsubsection{Iso-parametric finite elements}

We define parametric finite elements on a discrete surface $\Gamma_{h}^{d}$ which are piece-wise polynomial of degree $d$ or 1 over the reference simplex $\hat{T}$.

We define for $s \in\{1, d\}$

$$
W_{h}^{s}\left(\Gamma_{h}^{d}\right):=\left\{v_{h} \in \mathrm{C}^{0,1}\left(\Gamma_{h}^{d}\right) \mid \quad \Phi_{h}^{d} * v_{h}:=\left(v_{h} \circ \Phi_{T}^{d}\right) \in P_{s}(\hat{T}) \quad \forall T \in \mathcal{T}_{h}^{d}\right\} .
$$

The finite element space $W_{h}^{d}\left(\Gamma_{h}^{d}\right)$ is virtually iso-parametric as the local parametrisation of $\Gamma_{h}^{d}$ over the reference simplex is polynomial of degree $d$. In particular we have

$$
\operatorname{id}_{\Gamma_{h}^{d}} \in\left(W_{h}^{d}\left(\Gamma_{h}^{d}\right)\right)^{q}
$$

This justifies our notion of an "iso-parametric triangulation" introduced in the preceding section.

For given $s \in\{1, d\}$ the canonical nodal basis $\left\{v_{h}^{i} \mid i=1, \ldots, \operatorname{dim}\left(W_{h}^{s}\left(\Gamma_{h}^{d}\right)\right)\right\}$ of the finite element space $W_{h}^{s}\left(\Gamma_{h}^{d}\right)$ is defined by the images of the Lagrange-nodes of the reference element:

$$
\left\{Q_{i} \mid i=1, \ldots, \operatorname{dim}\left(W_{h}^{s}\left(\Gamma_{h}^{d}\right)\right)\right\}=\bigcup_{T \in \mathcal{T}_{h}^{d}}\left\{\Phi_{T}^{d}\left(\hat{Q}_{j}\right) \mid 1 \leq j \leq m(n, s)\right\} .
$$

The basis functions $v_{h}^{i}$ are then uniquely determined by requiring $v_{h}^{i}\left(Q_{j}\right)=\delta_{i j}$. We note that the first $(n+1)$ Lagrange-nodes $\hat{Q}_{1}, \ldots, \hat{Q}_{n+1}$ for any polynomial degree $d$ are the vertices of the reference element $\hat{T}$. This implies that it is possible to define the finite element space $W_{h}^{1}\left(\Gamma_{h}^{d}\right)$ of piecewise linear functions for any polynomial order $d \in \mathbb{N}$ of the parametrisation.

By Definition 1.3.25 on page 14 and the following remark 1.3 .10 we know that the Hilbert-space $\mathrm{H}^{1}\left(\Gamma_{h}^{d}\right)$ is well defined. It follows easily that $W_{h}^{s}<\mathrm{H}^{1}\left(\Gamma_{h}^{d}\right), s \in\{1, d\}$.

From [CIARLET and RAVIART 1972] we quote the following interpolation estimates:

\subsubsection{THEOREM}

Let $\Gamma \subset \mathbb{R}^{q}$ be a smooth compact immersed surface. Let $\mathrm{H}^{d+1}(\Gamma)$ be continuously embedded into $\mathrm{C}^{0}(\Gamma)$. By $I_{h}^{d}: \mathrm{H}^{d+1}(\Gamma) \rightarrow W_{h}^{d}\left(\Gamma_{h}^{d}\right)$ we denote the interpolation operator which interpolates a function $\varphi \in \mathrm{H}^{d+1}(\Gamma)$ in the Lagrange-nodes $\left\{Q_{i}\right\} \in \Gamma_{h}^{d}$. We note that $I_{h}^{d}$ is hereby uniquely determined. Further we denote by $\pi: \Gamma_{h}^{d} \rightarrow \Gamma$ the lift in normal direction with respect to $\Gamma$.

It then holds for any function $\varphi \in W_{h}^{d}\left(\Gamma_{h}^{d}\right)$ :

$$
\begin{aligned}
& \left\|\varphi-\pi\left(I_{h}^{d} \varphi\right)\right\|_{\mathrm{H}^{m}(\Gamma)} \leq c h^{d+1-m}\|\varphi\|_{\mathrm{H}^{d+1}(\Gamma)}, \quad \text { with } \quad m=0,1, \\
& \left\|\pi^{-1} \varphi-I_{h}^{d} \varphi\right\|_{\mathrm{H}^{m}(T)} \leq c h^{d+1-m}\left\|\pi^{-1} \varphi\right\|_{\mathrm{H}^{d+1}(T)}, \quad \text { with } \quad 0 \leq m \leq d+1 .
\end{aligned}
$$

Proof: [Ciarlet and Raviart 1972, Theorem 5]

REMARK 2.4.6

The regular iso-parametric $n$-simplex $T=\Phi_{T}^{d}(\hat{T}) \in \mathcal{T}_{h}^{d}$ is a $\mathrm{C}^{\infty}$-manifold. Thus the spaces $\mathrm{H}^{m}(T)$ are well defined and make sense for $0 \leq m \leq d+1$, where the upper bound $d+1$ is maximal in the sense that derivatives of order higher than $d$ of the local parametrisation $\Phi_{T}^{d}$ are constant. 


\subsubsection{A finite element formulation of the Newton iteration}

Based on the weak formulation (Definition 2.2.4 on page 35) of the scalar linearised Euler-Lagrange equations (2.24) we will now formulate a finite element discretisation of the continuous Newton iteration Algorithm 2.3.2 on page 40.

The goal is to approximate the solutions of the non-linear Euler-Lagrange equations (2.6) on page 23 in the context of iso-parametric discrete surfaces $\Gamma_{h}^{d} \subset \mathbb{R}^{3}$ of dimension 2 and their corresponding iso-parametric finite element spaces $W_{h}^{d}\left(\Gamma_{h}^{d}\right)$ as defined in the previous section. We will drop the upper index because we do not mix discretisations of different polynomial degree here, so for $d \in \mathbb{N}$ fixed we set $\Gamma_{h}:=\Gamma_{h}^{d}$ and $W_{h}\left(\Gamma_{h}\right):=W_{h}^{d}\left(\Gamma_{h}^{d}\right)$.

Moreover, the surfaces generated during the Newton iteration will be indexed by upper indices in brackets. So $\Gamma_{h}^{(l)}$ is the $l^{\text {th }}$ surface in the Newton iteration. In particular $(l)$ does not denote the polynomial degree of the parametrisation.

We begin by writing down the discrete analogon of the continuous iteration step Algorithm 2.3.2 on page 40 of the modified Newton iteration Algorithm 2.3.1 on page 38. The details will be discussed afterwards, see the Remarks 2.4 .7 to 2.4 .11 on pages $49-51$. Let $\Xi_{h}^{(l)}=\left(\operatorname{id}_{\Gamma_{h}^{(l)}}, C_{h}^{(l)}\right) \in\left(W_{h}\left(\Gamma_{h}^{(l)}\right)\right)^{3} \times \mathbb{R}$ be the point generated by the previous iteration step of a discretisation of the modified Newton algorithm. We use the following notations:

- $\Gamma_{h}^{(l)} \subset \mathbb{R}^{2} \times \mathbb{R}^{+}$is an iso-parametric surface with boundary $\Sigma_{h}^{(l)} \subset \mathbb{R}^{2} \times\{0\}$.

- We define the discrete residual $R_{h}^{(l)}$ of the non-linear Euler-Lagrange equations by the following expression:

$$
R_{h}^{(l)}:=2 H_{h}\left(\Gamma_{h}^{(l)}\right)-2 \omega^{2} r^{2}-C_{h}^{(l)} .
$$

The discrete mean curvature $H_{h}\left(\Gamma_{h}^{(l)}\right)$ is defined in Section 3.2, Definition 3.2.4 on page 62. We note that $R_{h}^{(l)} \in W_{h}\left(\Gamma_{h}^{(l)}\right)$ and refer to Remark 2.4.10 and Remark 2.4.11 on page 51 .

- We define a discrete version $\bar{a}_{h}^{(l)}$ of the $0^{\text {th }}$-order term $\bar{a}_{\Gamma}$ of the scalar linearised Euler-Lagrange equations:

$$
\bar{a}_{h}^{(l)}:=\left(I I_{h}\left(\Gamma_{h}^{(l)}\right): I I_{h}\left(\Gamma_{h}^{(l)}\right)\right)+8 \omega^{2}\left(x_{1} \nu_{1}+x_{2} \nu_{2}\right),
$$

where the discrete Weingarten-map $I I_{h}\left(\Gamma_{h}^{(l)}\right)$ is defined in Section 3.2, Definition 3.2.4 on page 62 .

- We remind of the notation $\bar{\Theta}:=\left(-x_{2}, \nu_{1}+x_{1} \nu 2\right) . \bar{\Theta}$ is generated by the action of the infinitesimal generator $\mathcal{R}$ of the symmetry group of rotations around the $x_{3}$-axis and is a solution of the scalar linearised Euler-Lagrange equations. $\bar{\Theta}$ is non-trivial when the cylinder-symmetry of the surface is broken, see equation (2.28) on page 31.

Next we generate $\Gamma_{h}^{(l+1)}$ and thereby $\Xi_{h}^{(l+1)}=\left(\operatorname{id}_{\Gamma_{h}^{(l+1)}}, C_{h}^{(l+1)}\right) \in\left(W_{h}\left(\Gamma_{h}^{(l+1)}\right)\right) \times \mathbb{R}$ using the following (sub-) steps as explained in Algorithm 2.4.1: 


\section{Algorithm 2.4.1 The iteration step of the discrete Newton iteration}

1: On $\Gamma_{h}^{(l)}$ solve the following system of equations for $\left(\bar{\psi}_{h}^{(l)}, K_{h}^{(l)}\right) \in W_{h}\left(\Gamma_{h}^{(l)}\right) \times \mathbb{R}$ and $\theta^{(l)} \in \mathbb{R}:$

$$
\begin{aligned}
\int_{\Gamma_{h}^{(l)}} \nabla_{\Gamma_{h}^{(l)}} \bar{\psi}_{h}^{(l)} \cdot \nabla_{\Gamma_{h}^{(l)}} & \bar{\eta}_{h} d o-\int_{\Gamma_{h}^{(l)}} \bar{a}_{h}^{(l)} \bar{\psi}_{h}^{(l)} \bar{\eta}_{h} d o \\
& -K_{h}^{(l)} \int_{\Gamma_{h}^{(l)}} \bar{\eta}_{h} d o-\theta^{(l)} \int_{\Gamma_{h}^{(l)}} \bar{\Theta} \bar{\eta}_{h} d o=\int_{\Gamma_{h}^{(l)}} R_{h}^{(l)} \bar{\eta}_{h} d o,
\end{aligned}
$$

$$
\begin{gathered}
\int_{\Gamma_{h}^{(l)}} \bar{\psi}_{h}^{(l)} d o=C_{h}^{(l)}, \\
\int_{\Gamma_{h}^{(l)}} \bar{\psi}_{h}^{(l)} \bar{\Theta} d o=0
\end{gathered}
$$

for all $\bar{\eta} \in W_{h}\left(\Gamma_{h}^{(l)}\right)$. See Remark 2.4.9 on the next page below.

2: Generate an orthogonal perturbation $\psi_{h}^{(l)} \in\left(W_{h}\left(\Gamma_{h}^{(l)}\right)\right)^{3}$ using an $\mathrm{L}_{2}$-projection with the appropriate boundary conditions. See also Remark 2.4.7 below.

$$
\left\{\begin{array}{l}
\int_{\Gamma_{h}^{(l)}} \psi_{h}^{(l)} \cdot \eta_{h} d o=\int_{\Gamma_{h}^{(l)}} \bar{\psi}_{h}^{(l)} \nu_{\Gamma_{h}^{(l)}} \cdot \eta_{h} d o, \\
\text { for all } \quad \eta_{h}=\left(\eta_{h}^{1}, \eta_{h}^{2}, \eta_{h}^{3}\right) \in\left(W_{h}\left(\Gamma_{h}^{(l)}\right)\right)^{3} \quad \text { with }\left.\quad \eta_{h}^{3}\right|_{\Sigma_{h}^{(l)}} \equiv 0, \\
\text { where } \quad \psi_{h}^{(l)}=\left(\psi_{h, 1}^{(l)}, \psi_{h, 2}^{(l)}, \psi_{h, 3}^{(l)}\right) \in\left(W_{h}\left(\Gamma_{h}^{(l)}\right)\right)^{3} \quad \text { with }\left.\quad \psi_{h, 3}^{(l)}\right|_{\Sigma_{h}^{(l)}} \equiv 0
\end{array}\right.
$$

3: Choose $\lambda_{l} \in \mathbb{R}^{+}$as explained in Algorithm 2.3.1 on page 38 (but see Remark 2.4.11 below) and set

$$
\Xi_{h}^{(l)}=\left(\operatorname{id}_{\Gamma_{h}^{(l+1)}}, C_{h}^{(l+1)}\right):=\left(\operatorname{id}_{\Gamma_{h}^{(l)}}, C_{h}^{(l)}\right)-\lambda_{l}\left(\psi_{h}^{(l)}, K_{h}^{(l+1)}\right),
$$

where

$$
\Gamma_{h}^{(l+1)}:=\left\{\operatorname{id}_{\Gamma_{h}^{(l)}}(X)-\lambda_{l} \psi_{h}^{(l)}(X) \mid X \in \Gamma_{h}^{(l)}\right\}=\left(\Gamma_{h}^{(l)}\right)_{-\lambda_{l} \psi_{h}^{(l)}}
$$

\section{REMARK 2.4.7 (PERTURBATIONS IN NORMAL DiRECTION)}

In step 2 of the discretised iteration step Algorithm 2.4.1 we have to use an $\mathrm{L}_{2}$-projection to construct a vector-valued perturbation because the normal-field $\nu_{\Gamma_{h}^{(l)}}$ is discontinuous across element boundaries. This means that $\nu_{\Gamma_{h}^{(l)}} \bar{\psi}_{h}^{(l)} \notin\left(W_{h}\left(\Gamma_{h}^{(l)}\right)\right)$.

Another possibility than using an $\mathrm{L}_{2}$-projection to construct a vector-valued perturbation in normal direction is to define a continuous approximate normal field $\nu_{h} \in\left(W_{h}\left(\Gamma_{h}^{(l)}\right)\right)^{3}$ and set $\psi_{h}^{(l)}:=\bar{\psi}_{h}^{(l)} \nu_{h}$. The construction of such an approximate normal field is discussed in Section 3.1 in the next chapter. 
Remark 2.4.8 (Polynomial Degree of the Finite Element space)

The discretised Newton iteration does not work for linearly discretised parametric surfaces. The reason is the $0^{\text {th }}$-order term $\bar{a}_{h}\left(\Gamma_{h}^{(l)}\right)$ which contains the square of the discrete Weingarten-map $I I_{h}\left(\Gamma_{h}^{(l)}\right)$. We present experimental convergence tests with respect to the $\mathrm{L}_{2^{-}}$and $\mathrm{L}_{\infty^{-}}$-norm for $I I_{h}$ in the Tables 3.1 to 3.4 on pages $65-68$ in the next chapter. The numerical experiments suggest that $I I_{h}$ converges with respect to the $\mathrm{L}_{2^{-}}$and $\mathrm{L}_{\infty}$-norm to the continues Weingarten-map of the underlying smooth surface if the polynomial degree of the interpolating iso-parametric surface is at least 2, but that we have no convergence in the linear case.

\section{REMARK 2.4.9 (BREAKING OF LiE-SyMmetries)}

1. For the continuous Newton iteration (Algorithm 2.3.2 on page 40) we were using an Ansatz-space of reflectively symmetric functions with respect to the $\left(x_{1}, x_{2}\right)$-plane to break the translational invariance of the linearised equations in $x_{3}$-direction.

To do so in the context of finite elements would imply to choose an iso-parametric triangulation which shares the reflective symmetry. But then an Ansatz-space consisting of reflectively symmetric functions would just result in a linear system with block-matrices composed of identical blocks, overlapping only for the nodes located in the equatorial plane. Exploiting the block-matrix structure to reduce the number of unknowns by roughly one half is equivalent to dissecting the drop at its equatorial plane and imposing the natural boundary conditions implied by the dissection.

For the system of scalar equations (2.42) which determine the signed length of the (discrete) orthogonal perturbation this implies homogenous Neumann boundary conditions.

2. Likewise, in the continuous setup the volume constraint as well as the transversality condition with respect to the infinitesimal rotations around the $x_{3}$-axis were resolved by using appropriate Ansatz-functions. This is not possible in the finiteelement regime because the goal is to use basis-functions with small simply connected support in order to generate sparse matrices. But it is in general not possible for such functions to satisfy integral constraints which prescribe global properties.

Therefore we have to use the transversality constraints (2.42b) and (2.42c) when solving of the discretised linear Euler-Lagrange equation (2.42). This is the reason why (2.42) is a saddle-point problem while the corresponding differential equation (2.34) in the first (sub-) step in the continuous scheme - Algorithm 2.3.2 on page 40 - is not.

REMARK 2.4.10 (EQUiVAlENCE OF THE Finite ELEMENT SPACES)

As introduced in Section 2.3.1 we use the notation

$$
\left(\Gamma_{h}\right)_{\psi_{h}}:=\left\{\operatorname{id}_{\Gamma_{h}}(X)+\psi_{h}(X) \mid X \in \Gamma_{h}\right\}
$$

with $\psi_{h} \in\left(W_{h}\left(\Gamma_{h}\right)\right)^{3}$. If $\left(\Gamma_{h}\right)_{\psi_{h}}$ is again a $\mathrm{C}^{0,1}$-immersion then it is also naturally an iso-parametric triangulated surface because $\operatorname{id}_{\Gamma_{h}}+\psi_{h}$ is piecewise a polynomial of degree $d$ over the reference element. This implies that the finite element spaces $W_{h}\left(\left(\Gamma_{h}\right)_{\psi_{h}}\right)$ and 
$W_{h}\left(\Gamma_{h}\right)$ are isomorphic and norm-equivalent with respect to the $\mathrm{H}^{1}$-norm. This is clear because by construction it holds for any $\varphi_{h} \in W_{h}\left(\Gamma_{h}\right)<\mathrm{C}^{0,1}\left(\Gamma_{h}\right)$ that $\varphi_{h} \in \mathrm{H}_{\infty}^{1}\left(\Gamma_{h}\right)$ and additionally $\varphi_{h}$ and $\Gamma_{h}$ are piecewise of class $\mathrm{C}^{\infty}$ (see [ADAMS 1975, Theorem 3.35]).

Therefore we can apply the chain-rule to the pull-back $\psi_{h} * f_{h}=f_{h} \circ \psi_{h}$ of any function $f_{h} \in W_{h}\left(\left(\Gamma_{h}\right)_{\psi_{h}}\right)$ and so we have $\psi_{h} * f_{h} \in W_{h}\left(\Gamma_{h}\right)$. In particular the notion of a weak-solution is equivalent for $W_{h}\left(\left(\Gamma_{h}\right)_{\psi_{h}}\right)$ and $W_{h}\left(\Gamma_{h}\right)$.

In the discrete regime there is - analogous to Remark 2.3.1 on page 40 - a chain of (weak) diffeomorphisms

$$
\Psi_{h}^{(l)}:=\operatorname{id}_{\Gamma_{h}^{(l)}}-\lambda_{l} \psi_{h}^{(l)} \in\left(W_{h}\left(\Gamma_{h}^{(l)}\right)\right)^{3}, l \in \mathbb{N}_{0}
$$

We can sketch the situation by the following diagram:

$$
\begin{aligned}
& \Gamma_{h}^{(0)} \quad \stackrel{\Psi_{h}^{(0)}}{\longrightarrow} \Gamma_{h}^{(1)} \quad \stackrel{\Psi_{h}^{(1)}}{\longrightarrow} \cdots \stackrel{\Psi_{h}^{(l-1)}}{\longrightarrow} \quad \Gamma_{h}^{(l)} \quad \stackrel{\Psi_{h}^{(l)}}{\longrightarrow} \ldots \\
& W_{h}\left(\Gamma_{h}^{(0)}\right) \stackrel{\Psi_{h}^{(0)} *}{\longleftarrow} W_{h}\left(\Gamma_{h}^{(1)}\right) \stackrel{\Psi_{h}^{(1)} *}{\longleftarrow} \ldots \stackrel{\Psi_{h}^{(l-1)} *}{\longleftarrow} W_{h}\left(\Gamma_{h}^{(l)}\right) \stackrel{\Psi_{h}^{(l)} *}{\longleftarrow} \ldots
\end{aligned}
$$

Analogous to the continuous case the equivalence between $\left.W_{h}\left(\Gamma_{h}^{(0)}\right)\right)$ and $W_{h}\left(\Gamma_{h}^{(l)}\right)$ is controlled by the $\mathrm{H}_{\infty}^{1}\left(\Gamma_{h}^{(0)}\right)$-norm of the chain of (weak) diffeomorphisms $\Psi_{h}^{(l-1)} \circ \cdots \circ \Psi_{h}^{(0)}$.

\section{REMARK 2.4.11 (STOPPING CRITERION)}

As stopping criterion for the discretised Newton method we use a norm of the discrete residual $R_{h}^{(l)}$ of the non-linear Euler-Lagrange equations as defined in equation (2.41).

The proper norm, of course, would be the operator norm of the right-hand side of the weak formulation (2.42) used in the iteration step (Algorithm 2.4.1 on page 49) above with respect to the finite element space $W_{h}\left(\Gamma_{h}^{(0)}\right)$.

From Remark 2.4.10 on the preceding page we see that the computation of this norm would be quite involved. Instead, we use a norm - which one is explained at the end of this remark - of $R_{h}^{(l)}$ with respect to $W_{h}\left(\Gamma_{h}^{(l)}\right)$. For the continuous case this practice is justified by the fact that we actually have a solution if the continuous Newton iteration converges and the residual of the non-linear Euler-Lagrange equations with respect to the limit surface is zero. So we can hope that a small discrete residual $R_{h}^{(l)}$ with respect to $W_{h}\left(\Gamma_{h}^{(l)}\right)$ indicates that we have arrived at a good approximation of a smooth drop-shape.

However, it is not clear that the norm of $R_{h}^{(l)}$ with respect to $W_{h}\left(\Gamma_{h}^{(l)}\right)$ can be used for the step-size control in Algorithm 2.3.1 on page 38 which determines the value of $\lambda_{l}$ in each iteration step. The step-size control is intended to enforce convergence when the initial value is not close to a solution.

Because the operator norm of the right-hand side of (2.42) with respect to the finite dimensional space $W_{h}\left(\Gamma_{h}^{(l)}\right)$ is just an Euclidean scalar-product we chose the Euclidean standard scalar-product with respect to the nodal basis of $W_{h}\left(\Gamma_{h}^{(l)}\right)$. 
REMARK 2.4.12 (SOLVERS FOR THE DISCRETE SYSTEM)

In the nodal basis of $W_{h}\left(\Gamma_{h}^{(l)}\right)$ implied by the Lagrange-nodes of the iso-parametric discrete surface $\Gamma_{h}^{(l)}$ the linear system (2.42) takes the form

$$
\left(\begin{array}{c|c}
A & B \\
\hline B^{\operatorname{tr}} & 0
\end{array}\right) \cdot\left[\begin{array}{l}
x \\
y
\end{array}\right]=\left[\begin{array}{l}
b \\
c
\end{array}\right],
$$

where $A \in \mathbb{R}^{N \times N}, B \in \mathbb{R}^{N \times 2}, x, b \in \mathbb{R}^{N}$ and $y, c \in \mathbb{R}^{2}$, given $N$ is the dimension of $W_{h}\left(\Gamma_{h}^{(l)}\right)$.

We note that $A$ itself is not invertible in general and not positive (semi-) definite. This means that a Schur-complement reformulation of (2.46) is in general not possible. In addition the non-definiteness of $A$ makes it necessary to use a solver for quadratic indefinite systems. We chose to use the symmlq-solver which is an iterative $C G$-like solver based on the Lanczos-algorithm, see [PAIGE and SAUnders 1975].

It is also not straight-forward to arrive at a good preconditioner, again because $A$ is indefinite. During our experiments we were using a level 1 ILU $(k)$-preconditioner for the system matrix as described in [BARRETT et al. 1994, pp. 74]. The important feature of an ILU $(k)$-preconditioner $S$ for $A$ is that the profile of $S$ depends only on the profile of $A$ but not on the values of the non-zero entries of $A$. Therefore a large part of the costly computations to generate the preconditioner can be shared between the iterationsteps of the Newton iteration. In addition we were using the Gibs-King algorithm which computes a permutation of the basis of $W_{h}\left(\Gamma_{h}^{(l)}\right)$ to minimise the fill-in of an $L U$ factorisation. Without such a permutation an $\operatorname{ILU}(k)$-preconditioner is computationally too costly and only reduces the number of iterations of the solver but does not reduce the computation time.

To be precise, if $S$ is an ILU(1)-preconditioner for $A$, then we were using the matrix

$$
\left(\begin{array}{l|l}
S & 0 \\
\hline 0 & 1
\end{array}\right)
$$

as a left-preconditioner for the entire system. Using the ILU(1)-preconditioner $S$ for $A$ is of course purely experimental. It appears that $S$ often is positive definite and can therefore be used as the matrix of a scalar product; otherwise it would not be straightforward to use it in the left-preconditioner above. The reason why $S$ is in many cases a good preconditioner for $A$ is probably that $A$ has only a few negative eigenvalues.

The general technique of preconditioning is for example explained in [BRAESS 1997] or again in [BARRETT et al. 1994].

\subsubsection{An error indicator}

In the course of the path-tracking algorithm the surface mesh is heavily deformed. We resolve the degeneration of the surface-elements implied by this deformation by the mesh-smoothing techniques described in the Section 3.4 of Chapter 3 below. 
To counterbalance the change in the local node-density which is even amplified by meshsmoothing (see Figure 3.11 on page 85) we use a local mesh-refinement strategy. The decision which elements should be refined or coarsened is governed by an ad-hoc error indicator which assigns a local error to each element of the iso-parametric triangulation. The indicator function we are using is modelled after an a posteriori error estimator derived by [VERFÜRTH 1994] for quasi-linear elliptic problems.

For an element $T \in \mathcal{T}_{h}$ with diameter $h_{T}$ the quantity $\eta_{T, 1}$ defined by

$$
\eta_{T, 1}^{2}:=C_{0}^{2} h_{T}^{2}\left\|-\Delta_{\Gamma_{h}} \mathrm{id}_{\Gamma_{h}}-\left(4 \omega^{2} r^{2}\right) \nu_{\Gamma_{h}}\right\|_{\mathrm{L}_{2}(T)}^{2}+C_{1}^{2} h_{T}\left\|\left[\left[\nu_{\Gamma_{h}}\right]\right]\right\|_{\mathrm{L}_{2}(\partial T)}^{2}
$$

is used as an indicator for the local $\mathrm{H}^{1}$-error. $C_{0}$ and $C_{1}$ are constants which can be chosen to adapt the error indicator to the real error for exactly known surfaces. [[.]] denotes the jump across element boundaries.

Modelled after an $\mathrm{L}_{2}$-estimator derived by [B̈̈NSCH and SIEBERT 1995] for semi-linear problems we can use $\eta_{T, 2}$ defined by

$$
\eta_{T, 2}^{2}:=C_{0}^{2} h_{T}^{4}\left\|-\Delta_{\Gamma_{h}} \mathrm{id}_{\Gamma_{h}}-\left(4 \omega^{2} r^{2}\right) \nu_{\Gamma_{h}}\right\|_{\mathrm{L}_{2}(T)}^{2}+C_{2}^{2} h_{T}^{3}\left\|\left[\left[\nu_{\Gamma_{h}}\right]\right]\right\|_{\mathrm{L}_{2}(\partial T)}^{2}
$$

as an ad-hoc $\mathrm{L}_{2}$-error-indicator.

Below in Chapter 4 we will compare the global error indicators

$$
\eta_{1}:=\sqrt{\sum_{T \in \mathcal{T}_{h}} \eta_{T, 1}^{2}} \text { and } \eta_{2}:=\sqrt{\sum_{T \in \mathcal{T}_{h}} \eta_{T, 2}^{2}}
$$

with the real $\mathrm{H}^{1}$ - and $\mathrm{L}_{2}$-errors of some approximate solutions generated by the Newton scheme.

For the adaption of the mesh with the error indicator $\eta_{T, 1}$ or $\eta_{T, 2}$ we were using the equal distribution strategy which aims at equally distributing the error over all mesh elements in the sense of squared mean values. This is explained in detail in [ERIKSON and JOHNSON 1991].

We remark that we leave the topology of the mesh unchanged during the discrete Newton iteration which generates the drop-shapes. It was our experience that mesh-adaption in between the iteration steps caused the Newton method to fail completely. We therefore adapt the mesh after the Newton iteration has terminated using the error indicators described above.

\subsection{The solving of the bifurcation equation}

\subsubsection{Finding of bifurcation points}

As a necessary condition for the existence of a bifurcation point in the sense of Definition 1.3.26 on page 15 the linearised Euler-Lagrange equation (2.23) on page 28 must have non-trivial solutions. Using the notations of the Newton iteration described in the 
previous sections this means that 0 is an eigenvalue of the Fréchet-derivative $L_{\omega}(\Xi)$ of the non-linear operator $A_{\omega}(\Xi)$ (assuming that $A$ is Fréchet-differentiable with respect to $\omega$ and $\Xi$ ). If $A_{\omega}$ is continuously Fréchet-differentiable then the spectrum of the linearisation depends continuously on $\omega$ and Theorem 1.3 .28 on page 15 guarantees that at a simple eigenvalue 0 of $L_{\omega}$ there is indeed a bifurcation point. Additionally, the same theorem establishes that the corresponding eigenfunction of $L_{\omega}$ approximates the drop-shapes of the new branch.

The remarks above motivate the Algorithm 2.5.1 for the finding of branch points and constructing an initial value for the path-tracking algorithm such that we can track down the new branch. The method arises naturally from the approximation property of the eigenfunctions corresponding to a 0 eigenvalue and was used before by others, see for example [Brown and SCRIVEN 1980a].

Algorithm 2.5.1 Flow-chart for the finding of new branches during the pathtracking algorithm. The adaptive computation of the drop shapes is illustrated in Algorithm 2.6.1 on page 58. $n^{-}$denotes the number of negative eigenvalues of the linearised operator (see (2.50) on the next page).

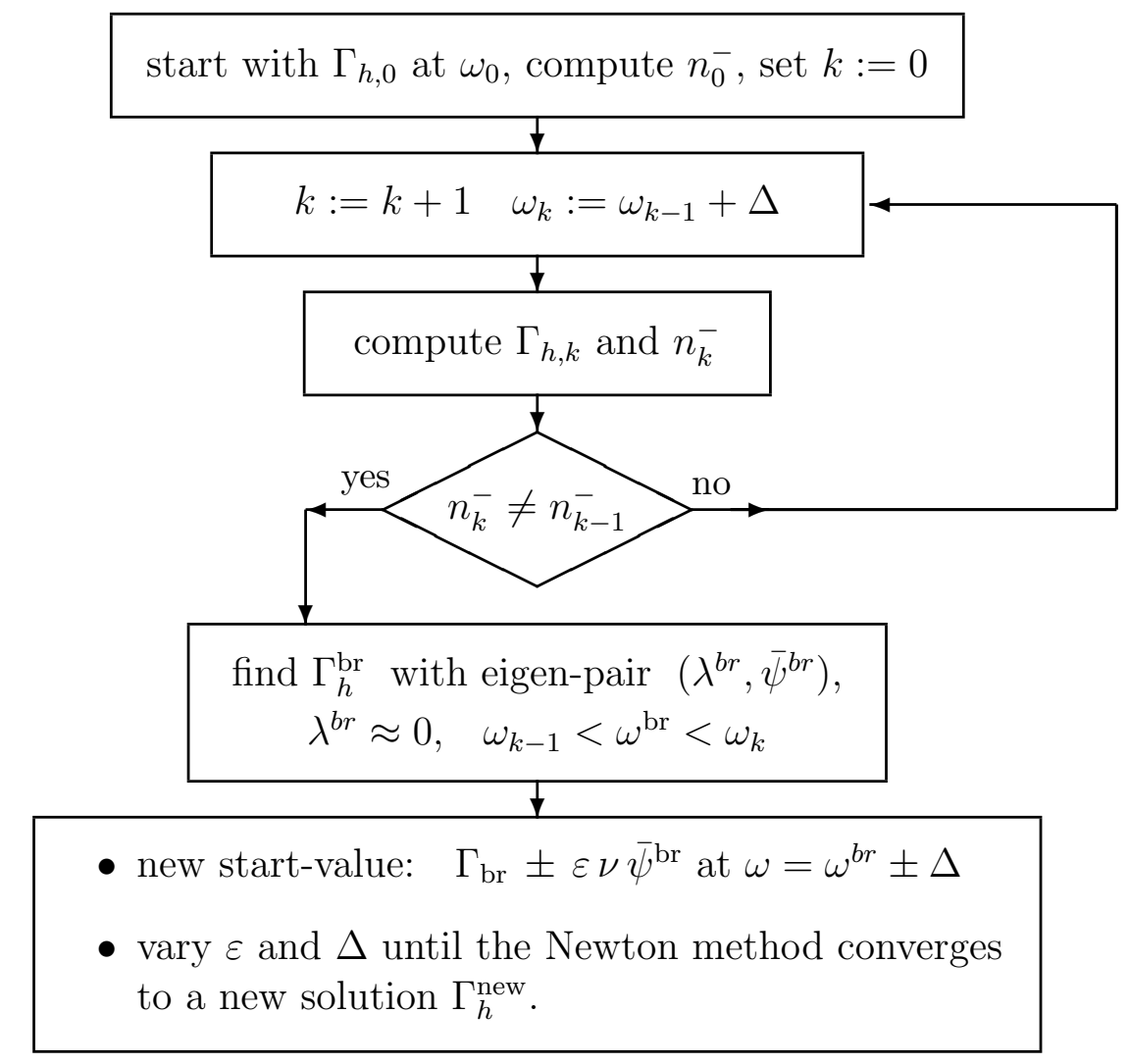

A central point of Algorithm 2.5.1 is the computation of all negative eigenvalues of the linearised operator. In the compact notation used above this means to find the smallest few eigenvalues of $L_{\omega}$ where the eigenvalues of $L_{\omega}$ are determined by solving the equation

$$
L_{\omega} \Xi=\lambda \Xi
$$


for $\Xi$ and $\lambda$. We remark that there are only finitely many negative eigenvalues because the spectrum of the Laplace-Beltrami operator $\Delta_{\Gamma}$ ( $\Gamma$ compact Riemannian $)$ is bounded from below, see [AUBIN 1982, Theorem 4.2].

Further, all (discrete) bifurcations we have found during our numerical experiments were bifurcations from "effectively" simple eigenvalues in the sense that occasionally a multiplicity of 2 was caused by the invariance of the linearised differential equation with respect to (infinitesimal) rotations around the $x_{3}$-axis.

Our final remark in this section is that Algorithm 2.5.1 on the preceding page is a "shooting"-method. If it doesn't find a new branch at a value of $\omega^{\text {br }}$ where an eigenvalue changes its sign then this does not imply that there is no branch-point. This is clear because the convergence of the Newton iteration depends on its initial value.

\subsubsection{The discretisation of the bifurcation equation}

For completeness we give here the discretisation of the eigenvalue equation (2.49) where we use the notations introduced in the construction of the discrete Newton iteration in the previous section. The discretisation of the eigenvalue equation follows from the first (sub-) step of the discrete Newton iteration-step Algorithm 2.4.1 on page 49. We assume that $\left(\Gamma_{h}, C_{h}\right) \equiv\left(\Gamma_{h, k}, C_{h, k}\right) \equiv \Xi_{h, k}$ is the $k^{\text {th }}$-surface constructed during the discrete path-tracking algorithm $(k \in \mathbb{N})$. So $\left(\Gamma_{h}, C_{h}\right)$ is an approximate limit of the discrete Newton method. We then arrive at the following discrete eigenvalue-problem which has to be solved for $\left(\bar{\psi}_{h}, K_{h}\right) \in W_{h}\left(\Gamma_{h}\right) \times \mathbb{R}, \theta \in \mathbb{R}$ :

$$
\left\{\begin{array}{l}
\int_{\Gamma_{h}} \nabla_{\Gamma_{h}} \bar{\psi}_{h} \cdot \nabla_{\Gamma_{h}} \bar{\eta}_{h} d o-\int_{\Gamma_{h}} \bar{a}_{h} \bar{\psi}_{h} \bar{\eta}_{h} d o \\
\quad-K_{h}^{(l)} \int_{\Gamma_{h}} \bar{\eta}_{h} d o-\theta^{(l)} \int_{\Gamma_{h}} \bar{\Theta} \bar{\eta}_{h} d o=\lambda \int_{\Gamma_{h}} \bar{\psi}_{h} \bar{\eta}_{h} d o \\
\int_{\Gamma_{h}} \bar{\psi}_{h} d o=0 \\
\int_{\Gamma_{h}} \bar{\psi}_{h} \bar{\Theta} d o=0 \\
\text { for all } \bar{\eta} \in W_{h}\left(\Gamma_{h}\right) .
\end{array}\right.
$$

See Remark 2.4.9 on page 50 for a discussion of the imposed constraints and the Neumann boundary conditions.

\subsubsection{Solvers for the constraint eigen-problem}

The matrices of the linear system (2.50) in the nodal basis of $W_{h}\left(\Gamma_{h}\right)$ implied by the Lagrange-nodes of the iso-parametric discrete surface $\Gamma_{h}$ are just the same as for the solution of the linear system in the discrete Newton method with the exceptions that the mass-matrix $M \in \mathbb{R}^{N \times N}$ appears on the right-hand side of the matrix equation. Using 
the same notation as for the matrix equation (2.46) on page 52 for the linear-system of the discrete Newton iteration-step we get the following generalised eigenvalue-problem:

$$
\left(\begin{array}{c|c}
A & B \\
\hline B^{\operatorname{tr}} & 0
\end{array}\right) \cdot\left[\begin{array}{l}
x \\
y
\end{array}\right]=\lambda\left(\begin{array}{c|c}
M & 0 \\
\hline 0 & 0
\end{array}\right)\left[\begin{array}{l}
x \\
y
\end{array}\right],
$$

where the unknowns are the eigenvalues $\lambda$ and the eigenvectors $(x, y)^{\operatorname{tr}}$.

[Brown and SCRIVEn 1980a] use a Cholesky decomposition of $A$ to transform (2.51) into a standard eigenvalue problem which then is solved using the Lanczos-algorithm. For large linear systems this leads to problems even when using a permutation to reduce the bandwidth of the Cholesky factorisation. The reason is that the matrices generated by a LU-factorisation from a sparse matrix are no longer sparse.

Apart from this the Cholesky decomposition seems to lead to problems for large systems in the sense that the solver for the standard eigenvalue problem failed to converge.

Another - possibility sometimes called "shift and invert" - is to choose an $s \in$ such that

$$
\left(\begin{array}{c|c}
A & B \\
\hline B^{\operatorname{tr}} & 0
\end{array}\right)-s\left(\begin{array}{c|c}
M & 0 \\
\hline 0 & 0
\end{array}\right)
$$

is invertible and use the inverse of this matrix to transform (2.51) into a standard eigenvalue problem. This method is described in detail in [LEHOUCQ et al. 1998].

For both methods it is essential to first compute a permutation of the nodal basis of $W_{h}\left(\Gamma_{h}\right)$ basis which minimises the fill-in of LU-factorisations. The permutation dramatically reduces the amount of memory needed for the Cholesky-factorisation and in the case of the "shift and invert" method it is needed for the ILU $(k)$-preconditioner in the inversion of the matrix, see our Remark 2.4.12 on page 52.

After converting the generalised eigenvalue problem (2.51) to standard form the Lanczos algorithm can be used to compute approximations to all negative eigenvalues and the corresponding eigenfunctions. For this purpose we have employed the ARPACK package, see [LEHOUCQ et al. 1998].

\subsection{The discrete path-tracking algorithm}

This last section of Chapter 2 gives finally an overview over the complete discrete pathtracking algorithm which is sketched in the flow-chart Algorithm 2.6.1 on page 58.

The goal is to compute a discrete set $\mathcal{S}_{h}=\left\{\left(\Gamma_{h}^{k}, C_{h}^{k}\right)\right\}$ of iso-parametric surfaces each approximating a smooth solution $\left(\Gamma^{k}, C^{k}\right)$ of the non-linear Euler-Lagrange equations (2.6) on page 23. $\mathcal{S}_{h}$ should have the property that, whenever $\left(\Gamma^{k}, C^{k}\right)$ and $\left(\Gamma^{k+1}, C^{k+1}\right)$ enclose a bifurcation point $\left(\Gamma^{\mathrm{br}}, C^{\mathrm{br}}\right)$, then $\left(\Gamma^{\mathrm{br}}, C^{\mathrm{br}}\right)$ as well as a discrete set of approximate solutions belonging to the new branch should also be part of $\mathcal{S}_{h}=\left\{\left(\Gamma_{h}^{k}, C_{h}^{k}\right)\right\}$. It is, of course, impossible to achieve this goal with our method (see our remark at the end of Section 2.5.1). 
The flow-chart Algorithm 2.6.1 on the following page illustrates the discrete pathtracking algorithm. A central part is the computation of the discrete drop-shapes. To generate a single new surface $\Gamma_{h}^{k+1}$ at a new angular velocity $\omega_{k+1}:=\omega_{k}+\Delta_{\omega}$ from its predecessor $\Gamma_{h}^{k}$ the following steps are needed:

- improve the regularity of the surface mesh by mesh-smoothing techniques, see Section 3.4 below,

- compute an intermediate new surface with the discrete Newton iteration, see Section 2.4,

- adapt the iso-parametric triangulation to the non-linear Euler-Lagrange equations (2.6) using the error indicators described in Section 2.4.4 - do this at least once even if the accumulated error indicator is below the given bound. The latter is used to counterbalance the redistribution of the nodes caused by the deformation of the mesh and by the mesh-smoothing.

This procedure is iterated until a given bound for the accumulated error indicator (see Section 2.4.4 above) is fulfilled.

Another point is that we have to adapt the step size $\Delta_{\omega}$ with respect to the angular velocity. If $\Delta_{\omega}$ is too large then the initial value for the Newton iteration is too far away from the new solution at $\omega_{k}+\Delta_{\omega}$ and the Newton iteration does not converge. We use a simple "divide by 2 and multiply by $\sqrt{2}$ " scheme as shown in the flow-chart Algorithm 2.6.1 on the next page.

The finding of bifurcation points was already described in Algorithm 2.5.1 on page 54 (Section 2.5). It searches for branch points and if it finds one, it tries to generate a new discrete surface $\Gamma_{h}^{\text {new }}$ which already belongs to the new branch. If it finds such a $\Gamma_{h}^{\text {new }}$ then a new instance of the path-tracking Algorithm 2.6.1 on the next page is started, this time with $\Gamma_{h}^{\text {new }}$ as initial surface.

The following Chapter 3 deals with numerical methods of iso-parametric triangulations in general and the last Chapter 4 deals with the results of our numerical experiments. 
Algorithm 2.6.1 Flow-chart for the adaptive computation of drop-shapes as part of the path-tracking algorithm. The finding of bifurcation points is illustrated in Algorithm 2.5.1 on page 54 .

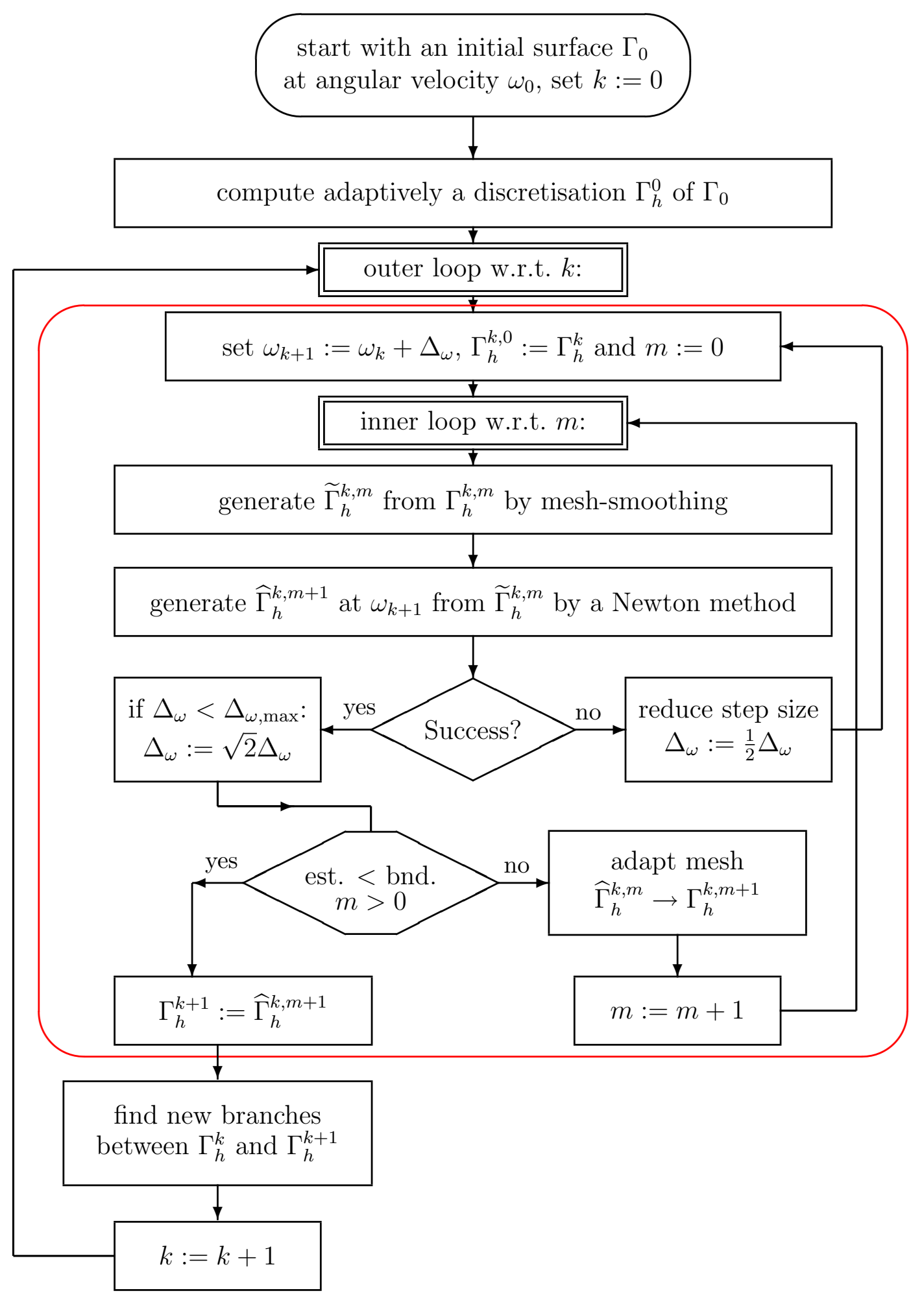




\section{Chapter 3}

\section{Numerical methods for iso-parametric meshes}

The implementation of the discretisation of the path-tracking algorithm described in the previous chapter was based on version 0.2 of the finite element toolbox ALBERT [SCHMIDT and SIEBERT 1998]. ALBERT provides abstract data-structures and library functions for the management of locally adaptive finite element algorithms on simplicial meshes in space-dimension 2 and 3. It supplies the user with predefined Lagrange finite element spaces up to polynomial degree 4, including the management of degrees of freedom, numerical quadrature and the abstract implementation of adaptive algorithms.

The treatment of parametric meshes was already well prepared to a certain extent, in particular the geometric dimension of the mesh-coordinates was separated from the dimension of the reference simplex. Based on the frame-work provided by the provisional version 0.2 of ALBERT we have implemented support for iso-parametric surfaces meshes up to arbitrary polynomial degree ALBERT supports.

This chapter is concerned with four important aspects which arise in the handling of isoparametric meshes without discussing the actual coding of the algorithms in the context of ALBERT.

In the first two sections we define discrete versions of geometric quantities, namely the outer unit normal field and - far more important - the notion of a discrete curvature for $\mathrm{C}^{0,1}$-surfaces.

The third Section 3.3 discusses in brief local mesh refinement and coarsening. In Section 3.4 we explain in greater detail mesh-smoothing techniques. Mesh-smoothing is a crucial point when using "moving-mesh" methods like our discretisation of the path-tracking algorithm in the previous chapter.

\subsection{Approximate normals}

For the implementation of the discrete Newton iteration as well as for the meshsmoothing algorithm we need a continuous approximate normal field for a discrete 
$n$-dimensional (hyper-) surface $\Gamma_{h}^{d} \subset \mathbb{R}^{n+1}$ and for it's linear interpolation $\Gamma_{h}^{1}$. In the following we can drop the upper index and set $\Gamma_{h}:=\Gamma_{h}^{d}$ and $W_{h}\left(\Gamma_{h}\right):=W_{h}^{d}\left(\Gamma_{h}^{d}\right)$.

[SCHMIDT 1993] defines a continuous approximate normal field by an averaging process effectively lumping the mass-matrix of the finite element space $W_{h}$ as is explained below.

At a Lagrange-node $P \in \Gamma_{h}$ we define an average normal $\nu_{h}^{a v}(P)$ as a weighted meanvalue of the element normals of its adjacent triangles,

$$
\tilde{\nu}_{h}^{a v}:=\frac{\sum_{T \in S(P)}|T| \nu_{T}}{\sum_{T \in S(P)}|T|}, \quad \nu_{h}^{a v}(P):=\frac{1}{\left\|\tilde{\nu}_{h}^{a v}(P)\right\|} \tilde{\nu}_{h}^{a v}(P),
$$

where $S(P)$ denotes the "star" around $P$ (meaning the set of elements adjacent to $P$ ) and $\nu_{T}$ the exact unit normal field of the iso-parametric $n$-simplex $T$. The approximate normal-field $\nu_{h}^{a v} \in W_{h}$ is then the Lagrange-interpolation between the values $\nu_{h}^{a v}(P)$ at the nodes of the iso-parametric triangulation $\mathcal{T}_{h}$.

[SCHMIDT 1993] remarks that we get the same value for $\nu_{h}^{a v}$ when defining it by the $\mathrm{L}_{2}$-projection of $\nu_{T}$ to $\left(W_{h}\left(\Gamma_{h}\right)\right)^{n+1}$

$$
\left(\tilde{\nu}_{h}^{\mathrm{L}_{2}}, \psi_{h}\right)_{\mathrm{L}_{2}\left(\Gamma_{h}\right)}:=\sum_{T \in \mathcal{T}_{h}}\left(\nu_{T}, \psi_{h}\right)_{\mathrm{L}_{2}(T)} \quad \forall \psi_{h} \in W_{h}\left(\Gamma_{h}\right), \quad \nu_{h}^{\mathrm{L}_{2}}:=\frac{1}{\left\|\tilde{\nu}_{h}^{\mathrm{L}_{2}}\right\|} \tilde{\nu}_{h}^{\mathrm{L}_{2}},
$$

if we use lumping for the mass-matrix on the left-hand side or a simple formula for the numerical quadrature on the left-hand side. So $\nu_{h}^{\mathrm{L}_{2}}=\nu_{h}^{a v}$ in this case. The topic of lumping and the associated error-estimates are described in [ThомÉE 1980].

In the presence of ill-shaped elements it might be better to use the angles $\angle(P, T)$ adjacent to the point $P$ as weight factor instead of the area $|T|$ of its adjacent elements (see the figure below for the notation):

$$
\tilde{\nu}_{h}^{\perp}(P):=\sum_{T \in S(P)} \angle(P, T) \nu_{T}, \quad \nu_{h}^{\angle}(P):=\frac{1}{\left\|\tilde{\nu}_{h}^{L}(P)\right\|} \tilde{\nu}_{h}^{\angle}(P) .
$$

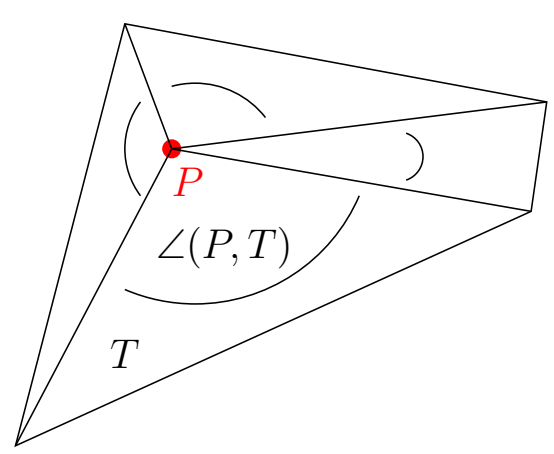

The difference between $\nu_{h}^{a v}, \nu_{h}^{\mathrm{L}_{2}}$ and $\nu_{h}^{<}$should be neglectable for regular-shaped iso-parametric triangulations. $\nu_{h}^{L}$ probably gives better results at the vertices $P$, because it stresses the fact that the direction of the normal at $P$ is a local property of the surface $\Gamma_{h}$ at $P$.

We note that the computational costs for the pathtracking algorithm described in the previous chapter are governed by the computation of the eigenvalues of the linearised Euler-Lagrange equations. This means that the computational costs of the three different averaging processes (3.1)-(3.3) are not an issue here.

We have implemented all three methods in the context of the finite element-toolbox ALBERT. Generally, we were using the exact $\mathrm{L}_{2}$-projection $\nu_{h}^{\mathrm{L}_{2}}$ as defined in equation 
(3.2) for the discretised Newton iteration described in the previous chapter and the angle-weighted averaged normals $\nu_{h}^{<}$of equation (3.3) in the projection step of the meshsmoothing algorithm, which is described in detail in Section 3.4.4.

We will not comment further on our particular choice and use the notation $\nu_{h}$ for either of the three possibilities.

\subsection{Curvature for Discrete Surfaces}

The linearisations (2.23) and (2.24) on page 28 of the Euler-Lagrange Equations (2.6) on page 23 contains the square sum $\kappa_{1}^{2}+\kappa_{2}^{2}=\operatorname{det} I I^{2}=2\left(2 H^{2}-K\right)$ of the principal curvatures of the surface $\Gamma$ of the drop. The present formulation of the Newton iteration needs a point-wise definition of this quantity as well as a point-wise definition of the mean-curvature for the computation of the residual in each Newton-step.

This would not be a problem for surfaces of class at least $C^{2}$. However, the surfaces $\Gamma_{h}^{n}$ of the discretised Newton iteration are only of class $C^{0,1}$. So it is not obvious how to define a good point-wise approximation for the curvature needed for the discretisation of the Newton-scheme.

In this section we define a discrete curvature for surfaces of class $C^{0,1}$. At the end we will present some experimental convergence tests for $C^{0,1}$-surfaces obtained by finite element approximations.

\subsubsection{Definition of the Discrete Curvature}

\subsubsection{Definition (PROJECTION)}

Let $W<X, X$ a vector space. $\Pi \in L(X, W)$ is called a projection from $X$ onto $W$, if $\Pi$ is surjective and idempotent.

3.2.2 LEMMA $\left(H^{-1}\right.$-PROJECTION)

Let $\Gamma$ be a compact $C^{0,1}$ manifold without boundary, $W<\mathrm{H}^{1}\left(\Gamma_{h}\right)$. Further, we denote by $\langle\langle\cdot, \cdot\rangle\rangle: \mathrm{H}^{-1}(\Gamma) \times \mathrm{H}^{1}(\Gamma) \rightarrow \mathbb{R}$ the dual pairing. Then the map

$$
\Pi: \mathrm{H}^{-1}(\Gamma) \rightarrow W
$$

defined by

$$
(\Pi v, \psi)_{\mathrm{L}_{2}(\Gamma)}=\langle\langle v, \psi\rangle\rangle \quad \forall \psi \in W
$$

is a projection from $\mathrm{H}^{-1}(\Gamma)$ onto $\left(W,\|\cdot\|_{\mathrm{L}_{2}(\Gamma)}\right)$.

Proof: This is clear because the $\mathrm{L}_{2}$-norm is weaker than the $\mathrm{H}_{2}^{1}$-norm.

REMARK 3.2.1 In general $\left(W,\|\cdot\|_{\mathrm{L}_{2}(\Gamma)}\right)$ is not a Hilbert-space even if $W<\mathrm{H}^{1}\left(\Gamma_{h}\right)$ is closed. 
For smooth $C^{2}$-surfaces $\Gamma \subset \mathbb{R}^{n+1}$ we have the identity

$$
Y_{\Gamma}=-\Delta_{\Gamma} \mathrm{id}_{\Gamma}, \quad \text { with } Y_{\Gamma}:=n H \nu .
$$

as was shown in Lemma 1.3.22 on page 13. So it follows for all $\psi \in\left(H^{1}(\Gamma)\right)^{n+1}$

$$
\int_{\Gamma} Y_{\Gamma} \cdot \psi d \Gamma=-\int_{\Gamma} \Delta_{\Gamma} \mathrm{id}_{\Gamma} \cdot \psi d \Gamma=\int_{\Gamma} \nabla_{\Gamma} \mathrm{id}_{\Gamma} \cdot \nabla_{\Gamma} \psi d \Gamma
$$

\subsubsection{Definition (Discrete Curvature Vector)}

Let $\Gamma_{h}$ be a $C^{0,1}$ approximation of a $C^{2}$ manifold $\Gamma \subset \mathbb{R}^{n+1}$ with $W_{h} \leq H^{1}\left(\Gamma_{h}\right)$ such that $\operatorname{id}_{\Gamma_{h}} \in\left(W_{h}\right)^{n+1}$. Then the discrete curvature vector $Y_{h} \in\left(W_{h}\right)^{n+1}$ is defined as the solution of

$$
\left(Y_{h}, \psi_{h}\right)_{\mathrm{L}_{2}\left(\Gamma_{h}\right)}=\left(\nabla_{\Gamma_{h}} \mathrm{id}_{\Gamma_{h}}, \nabla_{\Gamma_{h}} \psi_{h}\right)_{\mathrm{L}_{2}\left(\Gamma_{h}\right)} \quad \forall \psi_{h} \in\left(W_{h}\right)^{n+1}
$$

This definition follows [SCHMIDT 1993]. There it is shown that $Y_{h}$ is the $H^{-1}$ projection of the functional $-\Delta_{\Gamma_{h}} \mathrm{id}_{\Gamma_{h}} \in H^{-1}\left(\Gamma_{h}\right)$ defined by

$$
\left\langle\left\langle-\Delta_{\Gamma_{h}} \mathrm{id}_{\Gamma_{h}}, \psi\right\rangle\right\rangle:=-\left(\nabla_{\Gamma_{h}} \mathrm{id}_{\Gamma_{h}}, \nabla_{\Gamma_{h}} \psi\right)_{\mathrm{L}_{2}\left(\Gamma_{h}\right)} \quad \text { for } \quad \psi \in \mathrm{H}^{1}\left(\Gamma_{h}\right) .
$$

The experimental convergence tests for the case of a sphere presented in [SCHMIDT 1993] show approximate linear convergence in the $L^{2}$ and $L^{\infty}$ norms for quadratic Lagrange elements and no convergence for linear elements.

For the definition of the scalar mean and Gaussian curvature we have a look at the Weingarten map. Again, let $\Gamma$ be a smooth surface. Then the principal curvatures are the non-zero eigenvalues of the Weingarten map

$$
I I: \Gamma \rightarrow \mathbb{R}_{\mathrm{sym}}^{3 \times 3}: x \mapsto \nabla_{\Gamma} \nu_{\Gamma}(x),
$$

which is just the differential of the Gauß-map trivially extended to $T_{p} \Gamma^{\perp}$ and written in Cartesian coordinates, see Remark 1.3 .8 on page 12 .

To derive a weak formulation for $I I$ we multiply the equation $I I_{j}=\nabla_{\Gamma}\left(\nu_{\Gamma}\right)_{j}$ by a testfunction $\psi \in \mathrm{H}^{1}(\Gamma)$ and integrate over $\Gamma$. Then using the integration by parts formula (1.9) from on page 13 we arrive at

$$
\int_{\Gamma} I I_{j} \cdot \psi d o=\int_{\Gamma} \nabla_{\Gamma}\left(\nu_{\Gamma}\right)_{j} \cdot \psi d o=-\int_{\Gamma}(\nu)_{j} \nabla_{\Gamma} \cdot \psi d o-2 \int_{\Gamma} H\left(\nu_{\Gamma}\right)_{j} \psi \cdot \nu d o .
$$

We employ this relation to define a discrete Weingarten map $I I_{h}$ on a discrete surface $\Gamma_{h}$ :

\subsubsection{Definition (Discrete Weingarten map)}

Let $\Gamma_{h}$ be an $C^{0,1}$ approximation of a $C^{2}$ manifold $\Gamma \subset \mathbb{R}^{n+1}$ with $W_{h} \subset \mathrm{H}^{1}\left(\Gamma_{h}\right)$ such that $\operatorname{id}_{\Gamma_{h}} \in\left(W_{h}\right)^{n+1}$. Then the discrete Weingarten map $I I_{h} \in\left(W_{h}\right)^{(n+1) \times(n+1)}$ is defined as the solution of

$$
\begin{aligned}
& \left(\left(I I_{h}\right)_{j}, \psi_{h}\right)_{\mathrm{L}_{2}\left(\Gamma_{h}\right)}=-\left(\left(\nu_{h}\right)_{j}, \nabla_{\Gamma_{h}} \cdot \psi_{h}\right)_{\mathrm{L}_{2}\left(\Gamma_{h}\right)}-\left(\left(\nu_{h}\right)_{j}, Y_{h} \cdot \psi_{h}\right)_{\mathrm{L}_{2}\left(\Gamma_{h}\right)} \\
& \text { for all } \psi_{h} \in\left(W_{h}\right)^{n+1},
\end{aligned}
$$


where $Y_{h}$ is the discrete curvature vector defined in (3.4) and $\nu_{h}$ is the (discontinuous) normal field of $\Gamma_{h}$.

The discrete mean curvature $H_{h}$ and discrete Gaussian curvature $K_{h}$ (the latter only in the case of $n=2$ ) are defined by

$$
H_{h}:=\frac{1}{2} \operatorname{trace} I I_{h}, \quad K_{h}:=\operatorname{det}\left(I I_{h}+I_{3}\right)-2 H_{h}-1 .
$$

REMARK 3.2.2 $I I_{h} \in\left(W_{h}\right)^{(n+1) \times(n+1)}$ is the $\mathrm{H}^{-1}$ projection of the functional

$$
\nabla_{\Gamma_{h}} \nu_{h} \in\left(\mathrm{H}^{-1}\left(\Gamma_{h}\right)\right)^{(n+1) \times(n+1)}
$$

defined by

$$
\left\langle\left\langle\nabla_{\Gamma_{h}} \nu_{h}, \psi\right\rangle\right\rangle:=-\left(\left(\nu_{h}\right)_{j}, \nabla_{\Gamma_{h}} \cdot \psi\right)_{\mathrm{L}_{2}\left(\Gamma_{h}\right)}-\left(\left(\nu_{h}\right)_{j}, Y_{h} \cdot \psi\right)_{\mathrm{L}_{2}\left(\Gamma_{h}\right)} \quad \forall \psi \in\left(\mathrm{H}^{1}\left(\Gamma_{h}\right)\right)^{n+1} .
$$

\subsubsection{Convergence tests}

Figure 3.1 Surface meshes for testing our definition of the discrete curvature. The defining equations for the surfaces are $3.7 \mathrm{~b}$ to $3.7 \mathrm{~d}$ on this page. A picture for $3.7 \mathrm{a}$ has been omitted as it is of the same type as the surface shown in (a), but with smaller Gaussian curvature.

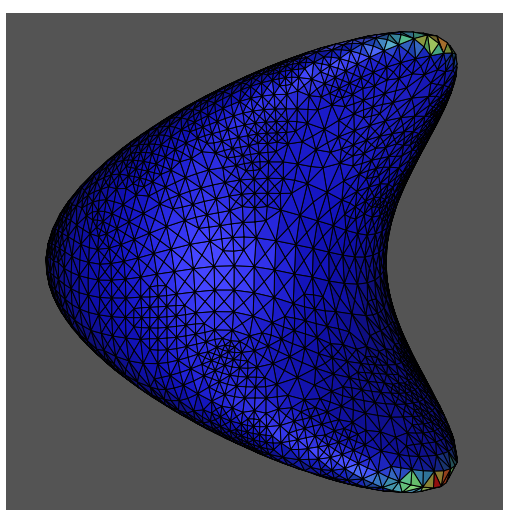

(a) Equation (3.7b)

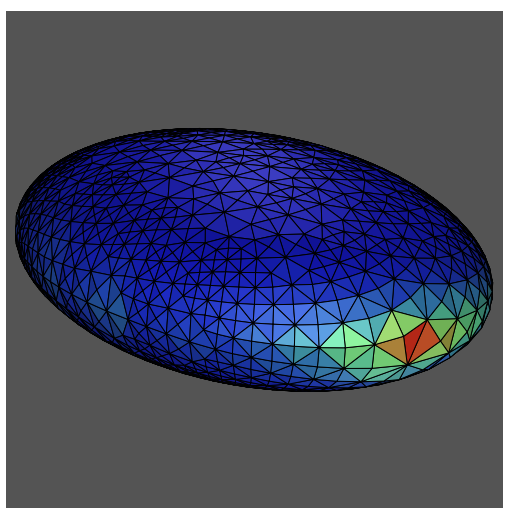

(b) Equation (3.7c)

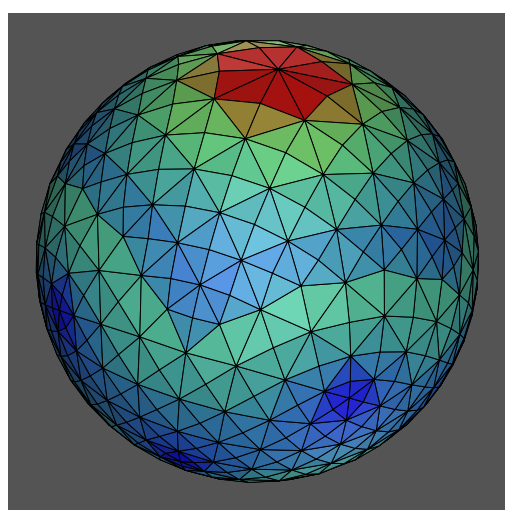

(c) Equation (3.7d)

Table 3.1 to 3.4 on pages $65-68$ show the results of our experimental convergence tests with piecewise polynomial discretisations of degree 1, 2, 3 and 4 for the following model problems (see Figure 3.1):

$$
\begin{gathered}
\left(x-z^{2}\right)^{2}+y^{2}+z^{2}-1=0 \\
\left(x-z^{2}\right)^{2}+\left(y-z^{2}\right)^{2}+z^{2}-1=0 \\
x^{2}+(2 y)^{2}+\left(\frac{2}{3} z\right)^{2}-1=0 \\
x^{2}+y^{2}+z^{2}-1=0
\end{gathered}
$$


The underlying macro triangulation for the discretisation of all model-problems is an icosahedron. The discrete surface $\Gamma_{h}$ is just the interpolation of the exact shape $\Gamma$ with Lagrange-polynomials. $\Gamma$ is viewed as a graph in approximate normal direction over the discrete surface $\Gamma_{h}$.

At first the mesh was refined adaptively until the $\mathrm{L}_{2}$ error with respect to the exact surface was below 0.005 . Afterwards the meshes were refined globally by doubly bisecting the elements in each refinement step.

The tables 3.1 to 3.4 on pages $65-68$ show the number of unknowns, the maximal mesh width, the relative $\mathrm{L}_{2}$ and $\mathrm{L}_{\infty}$ errors and the experimental order of convergence $(E O C)$ of the discrete mean and Gaussian curvatures $H_{h}$ and $K_{h}$ as defined in (3.6).

For the normalisation of the relative errors we use the curvature of the exact surfaces defined by $3.7 \mathrm{a}$ to $3.7 \mathrm{~d}$ on the page before:

$$
\mathcal{E}_{C, r}:=\frac{\left\|C-C_{h}\right\|_{\mathrm{L}_{r}\left(\Gamma_{h}\right)}}{\|C\|_{\mathrm{L}_{r}\left(\Gamma_{h}\right)}}, \quad \text { where } C \in\{K, H\}, \quad r \in\{2, \infty\} .
$$

As usual, the experimental order of convergence is defined as

$$
E O C:=\frac{\log \frac{\mathcal{E}_{j}}{\mathcal{E}_{j+1}}}{\log \frac{h_{j}}{h_{j+1}}} .
$$

While it is not surprising that the discrete curvatures originating from Definition 3.2.4 on page 62 do not converge for linearly interpolated surfaces, the experimental order of convergence for higher order parametrisations is surprising.

The tables 3.1 to 3.4 on pages $65-68$ suggest that for Lagrange-interpolates of polynomial degree $k$ the experimental order of convergence for the discrete curvature is greater than $k-1$, provided that $k>1$.

It should be stressed that we did not interpolate the curvatures of the $C^{\infty}$ surfaces defined by 3.1 to 3.4 on pages $65-68$ with finite elements, but just the surfaces themselves. This means that the errors shown in Table 3.1 to 3.4 on pages $65-68$ are not mere interpolation errors. We would have expected values for the $E O C$ of at most $k-1$, but not greater.

It should also be stressed that the results for the less complicated surfaces (3.7c) (an ellipsoid) and (3.7d) (a sphere) (see Table 3.3 on page 67 and 3.4 on page 68) are not as suggestive as the results for the more complicated surfaces. Table 3.3 on page 67 and 3.4 on page 68 show a decreasing $E O C$ as the number of unknowns increases.

This might indicate that the values for the $E O C$ as shown in the tables 3.1 on the next page and 3.2 on page 66 just did not stabilise yet.

We presume that it is sufficient to use Lagrange finite elements of order 2 for the parametrisation of the discretised surfaces; even with respect to the $\mathrm{L}_{\infty}$-norm the discretised curvature of the interpolating surface seems to converge to the exact curvature of the $C^{\infty}$ surface.

To our knowledge no analytical results are known with respect to this problem. 
Table 3.1 Discrete curvature, problem 1, relative errors

Polynomial degree: 1

\begin{tabular}{|r||l||l|l||l|c||c|c||c|c||}
\hline \#unkn. & $h_{\max }$ & $\mathcal{E}_{K, 2}$ & EOC & $\mathcal{E}_{K, \infty}$ & EOC & $\mathcal{E}_{H, 2}$ & EOC & $\mathcal{E}_{H, \infty}$ & EOC \\
\hline \hline 2593 & 0.135 & 0.79 & $\cdot$ & 1.4 & $\cdot$ & 0.19 & $\cdot$ & 0.36 & $\cdot$ \\
\hline 10366 & 0.0689 & 0.87 & -0.16 & 2.2 & -0.61 & 0.19 & 0.055 & 0.36 & -0.028 \\
\hline 41458 & 0.0361 & 0.9 & -0.041 & 2.1 & 0.015 & 0.19 & -0.0011 & 0.4 & -0.15 \\
\hline 165826 & 0.0199 & 0.91 & -0.022 & 2.3 & -0.12 & 0.19 & -0.0065 & 0.42 & -0.081 \\
\hline 663298 & 0.0114 & 0.91 & -0.0072 & 2.3 & -0.02 & 0.19 & -0.0042 & 0.43 & -0.024 \\
\hline 2653186 & 0.00654 & 0.91 & -0.0023 & 2.3 & -0.0059 & 0.19 & -0.0021 & 0.43 & -0.0092 \\
\hline
\end{tabular}

Polynomial degree: 2

\begin{tabular}{|r||l||l|l||l|l||l|l||l|l||}
\hline \#unkn. & $h_{\max }$ & $\mathcal{E}_{K, 2}$ & $\mathrm{EOC}$ & $\mathcal{E}_{K, \infty}$ & EOC & $\mathcal{E}_{H, 2}$ & EOC & $\mathcal{E}_{H, \infty}$ & EOC \\
\hline \hline 522 & 0.372 & 1.7 &. & 2.5 &. & 0.15 &. & 0.48 &. \\
\hline 2082 & 0.206 & 1. & 0.85 & 2.7 & -0.14 & 0.085 & 0.92 & 0.31 & 0.75 \\
\hline 8322 & 0.118 & 0.54 & 1.2 & 1.5 & 1. & 0.044 & 1.2 & 0.15 & 1.3 \\
\hline 33282 & 0.0681 & 0.2 & 1.8 & 0.69 & 1.4 & 0.02 & 1.4 & 0.067 & 1.5 \\
\hline 133122 & 0.0382 & 0.082 & 1.6 & 0.44 & 0.78 & 0.0099 & 1.3 & 0.044 & 0.74 \\
\hline 532482 & 0.0219 & 0.032 & 1.7 & 0.13 & 2.2 & 0.0047 & 1.3 & 0.018 & 1.6 \\
\hline 2129922 & 0.0123 & 0.015 & 1.4 & 0.044 & 1.9 & 0.0023 & 1.2 & 0.0088 & 1.2 \\
\hline
\end{tabular}

Polynomial degree: 3

\begin{tabular}{|r||l||l|c||l|c||l|l||l|c||}
\hline \#unkn. & $h_{\max }$ & $\mathcal{E}_{K, 2}$ & $\mathrm{EOC}$ & $\mathcal{E}_{K, \infty}$ & EOC & $\mathcal{E}_{H, 2}$ & EOC & $\mathcal{E}_{H, \infty}$ & EOC \\
\hline \hline 848 & 0.488 & 1.5 &. & 3.6 &. & 0.13 &. & 0.49 &. \\
\hline 3386 & 0.32 & 2.1 & -0.83 & 5.4 & -1. & 0.15 & -0.26 & 0.58 & -0.4 \\
\hline 13538 & 0.191 & 1.1 & 1.1 & 6.3 & -0.3 & 0.087 & 1. & 0.62 & -0.12 \\
\hline 54146 & 0.11 & 0.17 & 3.5 & 1.3 & 2.9 & 0.022 & 2.5 & 0.17 & 2.3 \\
\hline 216578 & 0.064 & 0.054 & 2.1 & 0.46 & 1.9 & 0.0068 & 2.1 & 0.083 & 1.4 \\
\hline 866306 & 0.0359 & 0.014 & 2.3 & 0.13 & 2.2 & 0.0018 & 2.3 & 0.026 & 2. \\
\hline 3465218 & 0.0202 & 0.0036 & 2.4 & 0.034 & 2.3 & 0.00046 & 2.4 & 0.0069 & 2.3 \\
\hline
\end{tabular}

Polynomial degree: 4

\begin{tabular}{|r||l||l|l||l|l||l|l||l|l||}
\hline \#unkn. & $h_{\max }$ & $\mathcal{E}_{K, 2}$ & EOC & $\mathcal{E}_{K, \infty}$ & EOC & $\mathcal{E}_{H, 2}$ & EOC & $\mathcal{E}_{H, \infty}$ & EOC \\
\hline \hline 754 & 0.549 & 4.5 &. & 13. &. & 0.3 &. & 1.4 &. \\
\hline 3010 & 0.324 & 1.6 & 2. & 8.6 & 0.77 & 0.12 & 1.8 & 0.75 & 1.2 \\
\hline 12034 & 0.196 & 0.34 & 3.1 & 1.5 & 3.5 & 0.035 & 2.4 & 0.24 & 2.3 \\
\hline 48130 & 0.121 & 0.04 & 4.4 & 0.5 & 2.2 & 0.0048 & 4.1 & 0.055 & 3. \\
\hline 192514 & 0.0697 & 0.0047 & 3.9 & 0.046 & 4.3 & 0.00074 & 3.4 & 0.0092 & 3.2 \\
\hline 770050 & 0.0402 & 0.00061 & 3.7 & 0.0062 & 3.6 & $9.1 e-05$ & 3.8 & 0.0012 & 3.7 \\
\hline 3080194 & 0.0232 & $7.4 e-05$ & 3.8 & 0.00068 & 4. & $1.1 e-05$ & 3.8 & 0.00015 & 3.8 \\
\hline \hline
\end{tabular}


Table 3.2 Discrete curvature, problem 2, relative errors

Polynomial degree: 1

\begin{tabular}{|r||l||l|c||c|c||c|c||c|c||}
\hline \#unkn. & $h_{\max }$ & $\mathcal{E}_{K, 2}$ & EOC & $\mathcal{E}_{K, \infty}$ & EOC & $\mathcal{E}_{H, 2}$ & EOC & $\mathcal{E}_{H, \infty}$ & EOC \\
\hline \hline 2673 & 0.205 & 1.7 &. & 1.9 &. & 0.39 &. & 0.69 &. \\
\hline 10686 & 0.0957 & 1.4 & 0.22 & 1.7 & 0.14 & 0.24 & 0.64 & 0.59 & 0.21 \\
\hline 42738 & 0.0508 & 1.5 & -0.11 & 2.3 & -0.44 & 0.2 & 0.29 & 0.37 & 0.75 \\
\hline 170946 & 0.0263 & 1.7 & -0.19 & 3.3 & -0.56 & 0.19 & 0.057 & 0.37 & -0.025 \\
\hline 683778 & 0.0151 & 1.8 & -0.091 & 3.7 & -0.21 & 0.19 & -0.0025 & 0.41 & -0.17 \\
\hline 2735106 & 0.00864 & 1.8 & -0.033 & 3.7 & -0.037 & 0.2 & -0.0041 & 0.43 & -0.085 \\
\hline
\end{tabular}

Polynomial degree: 2

\begin{tabular}{|r||l||l|c||c|c||l|l||l|l||}
\hline \#unkn. & $h_{\max }$ & $\mathcal{E}_{K, 2}$ & EOC & $\mathcal{E}_{K, \infty}$ & EOC & $\mathcal{E}_{H, 2}$ & EOC & $\mathcal{E}_{H, \infty}$ & EOC \\
\hline \hline 970 & 0.368 & 2.7 &. & 5.2 &. & 0.25 &. & 0.58 &. \\
\hline 3874 & 0.204 & 4.4 & -0.83 & 11. & -1.3 & 0.15 & 0.9 & 0.42 & 0.52 \\
\hline 15490 & 0.116 & 2.4 & 1.1 & 6.4 & 1. & 0.073 & 1.2 & 0.31 & 0.53 \\
\hline 61954 & 0.0673 & 0.96 & 1.7 & 3.8 & 0.95 & 0.039 & 1.2 & 0.21 & 0.71 \\
\hline 247810 & 0.0379 & 0.34 & 1.8 & 1.6 & 1.5 & 0.018 & 1.3 & 0.087 & 1.6 \\
\hline 991234 & 0.0214 & 0.11 & 2. & 0.47 & 2.2 & 0.0085 & 1.4 & 0.039 & 1.4 \\
\hline
\end{tabular}

Polynomial degree: 3

\begin{tabular}{|r||l||l|c||l|c||l|l||l|c||}
\hline \#unkn. & $h_{\max }$ & $\mathcal{E}_{K, 2}$ & $\mathrm{EOC}$ & $\mathcal{E}_{K, \infty}$ & $\mathrm{EOC}$ & $\mathcal{E}_{H, 2}$ & $\mathrm{EOC}$ & $\mathcal{E}_{H, \infty}$ & EOC \\
\hline \hline 1559 & 0.468 & 12. &. & 19. &. & 0.45 &. & 0.84 &. \\
\hline 6230 & 0.322 & 13. & -0.15 & 29. & -1.1 & 0.34 & 0.73 & 1.1 & -0.78 \\
\hline 24914 & 0.19 & 10. & 0.39 & 50. & -1.1 & 0.19 & 1.2 & 1.4 & -0.4 \\
\hline 99650 & 0.113 & 1.3 & 4. & 8. & 3.5 & 0.055 & 2.3 & 0.43 & 2.3 \\
\hline 398594 & 0.0653 & 0.36 & 2.3 & 3.6 & 1.4 & 0.022 & 1.7 & 0.27 & 0.82 \\
\hline 1594370 & 0.0373 & 0.086 & 2.6 & 0.88 & 2.5 & 0.0062 & 2.2 & 0.1 & 1.8 \\
\hline
\end{tabular}

Polynomial degree: 4

\begin{tabular}{|r||l||l|l||l|l||l|l||l|c||}
\hline \#unkn. & $h_{\max }$ & $\mathcal{E}_{K, 2}$ & EOC & $\mathcal{E}_{K, \infty}$ & EOC & $\mathcal{E}_{H, 2}$ & EOC & $\mathcal{E}_{H, \infty}$ & EOC \\
\hline \hline 1874 & 0.477 & 15. &. & 39. &. & 0.45 &. & 1.2 &. \\
\hline 7490 & 0.304 & 8. & 1.4 & 58. & -0.87 & 0.22 & 1.6 & 1.3 & -0.16 \\
\hline 29954 & 0.196 & 1.2 & 4.3 & 9.2 & 4.2 & 0.055 & 3.2 & 0.45 & 2.5 \\
\hline 119810 & 0.115 & 0.22 & 3.3 & 3.1 & 2. & 0.013 & 2.7 & 0.14 & 2.2 \\
\hline 479234 & 0.0655 & 0.022 & 4.1 & 0.34 & 3.9 & 0.0019 & 3.5 & 0.026 & 3. \\
\hline 1916930 & 0.0374 & 0.0024 & 3.9 & 0.025 & 4.7 & 0.00023 & 3.8 & 0.0032 & 3.7 \\
\hline
\end{tabular}


Table 3.3 Discrete curvature, problem 3, relative errors

Polynomial degree: 1

\begin{tabular}{|r||l||l|l||l|c||c|c||c|c||}
\hline \#unkn. & $h_{\max }$ & $\mathcal{E}_{K, 2}$ & EOC & $\mathcal{E}_{K, \infty}$ & EOC & $\mathcal{E}_{H, 2}$ & EOC & $\mathcal{E}_{H, \infty}$ & EOC \\
\hline \hline 1799 & 0.2 & 0.76 & $\cdot$ & 1.4 & $\cdot$ & 0.19 & $\cdot$ & 0.45 & $\cdot$ \\
\hline 7190 & 0.117 & 0.83 & -0.14 & 1.7 & -0.39 & 0.18 & 0.056 & 0.39 & 0.25 \\
\hline 28754 & 0.0674 & 0.83 & -0.0022 & 1.7 & 0.028 & 0.18 & 0.019 & 0.43 & -0.2 \\
\hline 115010 & 0.0384 & 0.83 & -0.0086 & 1.7 & -0.098 & 0.18 & -0.0021 & 0.48 & -0.17 \\
\hline 460034 & 0.0218 & 0.83 & -0.0067 & 1.7 & 0.0033 & 0.18 & -0.0034 & 0.54 & -0.2 \\
\hline 1840130 & 0.0127 & 0.84 & -0.0037 & 1.8 & -0.012 & 0.18 & -0.0019 & 0.59 & -0.19 \\
\hline
\end{tabular}

Polynomial degree: 2

\begin{tabular}{|r||l||l|c||l|c||c|c||c|c||}
\hline \#unkn. & $h_{\max }$ & $\mathcal{E}_{K, 2}$ & EOC & $\mathcal{E}_{K, \infty}$ & EOC & $\mathcal{E}_{H, 2}$ & EOC & $\mathcal{E}_{H, \infty}$ & EOC \\
\hline \hline 514 & 0.295 & 0.69 &. & 1.6 &. & 0.086 &. & 0.27 &. \\
\hline 2050 & 0.19 & 0.49 & 0.77 & 1.7 & -0.23 & 0.06 & 0.81 & 0.23 & 0.41 \\
\hline 8194 & 0.107 & 0.21 & 1.5 & 1.1 & 0.85 & 0.026 & 1.4 & 0.12 & 1.1 \\
\hline 32770 & 0.0588 & 0.069 & 1.8 & 0.47 & 1.4 & 0.011 & 1.5 & 0.063 & 1.1 \\
\hline 131074 & 0.0309 & 0.025 & 1.6 & 0.17 & 1.6 & 0.0049 & 1.2 & 0.031 & 1.1 \\
\hline 524290 & 0.0159 & 0.011 & 1.3 & 0.056 & 1.7 & 0.0024 & 1.1 & 0.013 & 1.3 \\
\hline 2097154 & 0.00833 & 0.0051 & 1.2 & 0.026 & 1.2 & 0.0012 & 1.1 & 0.0064 & 1.1 \\
\hline
\end{tabular}

Polynomial degree: 3

\begin{tabular}{|r||l||l|l||l|l||l|l||l|l||}
\hline \#unkn. & $h_{\max }$ & $\mathcal{E}_{K, 2}$ & $\mathrm{EOC}$ & $\mathcal{E}_{K, \infty}$ & $\mathrm{EOC}$ & $\mathcal{E}_{H, 2}$ & $\mathrm{EOC}$ & $\mathcal{E}_{H, \infty}$ & EOC \\
\hline \hline 1082 & 0.309 & 2.2 &. & 8.3 &. & 0.23 &. & 1. &. \\
\hline 4322 & 0.198 & 0.76 & 2.3 & 3.4 & 2. & 0.09 & 2.1 & 0.59 & 1.2 \\
\hline 17282 & 0.131 & 0.16 & 3.8 & 1.2 & 2.6 & 0.025 & 3.1 & 0.19 & 2.8 \\
\hline 69122 & 0.0788 & 0.041 & 2.7 & 0.31 & 2.6 & 0.0067 & 2.6 & 0.063 & 2.1 \\
\hline 276482 & 0.0457 & 0.011 & 2.5 & 0.086 & 2.4 & 0.0017 & 2.5 & 0.019 & 2.2 \\
\hline 1105922 & 0.0259 & 0.0027 & 2.4 & 0.024 & 2.2 & 0.00044 & 2.4 & 0.0052 & 2.3 \\
\hline 4423682 & 0.0145 & 0.00067 & 2.4 & 0.0063 & 2.3 & 0.00011 & 2.4 & 0.0014 & 2.3 \\
\hline
\end{tabular}

Polynomial degree: 4

\begin{tabular}{|r||l||l|l||l|l||c|c||l|r||}
\hline \#unkn. & $h_{\max }$ & $\mathcal{E}_{K, 2}$ & $\mathrm{EOC}$ & $\mathcal{E}_{K, \infty}$ & EOC & $\mathcal{E}_{H, 2}$ & EOC & $\mathcal{E}_{H, \infty}$ & EOC \\
\hline \hline 978 & 0.443 & 7.5 &. & 72. &. & 0.39 &. & 4.1 &. \\
\hline 3906 & 0.265 & 0.23 & 6.8 & 2.5 & 6.5 & 0.04 & 4.4 & 0.36 & 4.7 \\
\hline 15618 & 0.153 & 0.024 & 4.1 & 0.24 & 4.3 & 0.0056 & 3.6 & 0.065 & 3.1 \\
\hline 62466 & 0.0908 & 0.0027 & 4.2 & 0.027 & 4.2 & 0.00063 & 4.2 & 0.0059 & 4.6 \\
\hline 249858 & 0.0527 & 0.00034 & 3.8 & 0.004 & 3.5 & $8 e-05$. & 3.8 & 0.0012 & 2.9 \\
\hline 999426 & 0.0297 & $4.3 e-05$ & 3.6 & 0.00057 & 3.4 & $1 e-05$. & 3.6 & 0.00018 & 3.4 \\
\hline 3997698 & 0.0168 & $5.5 e-06$ & 3.6 & $9.9 e-05$ & 3.1 & $1.3 e-06$ & 3.6 & $2.8 e-05$ & 3.2 \\
\hline \hline
\end{tabular}


Table 3.4 Discrete curvature, problem 4, relative errors

Polynomial degree: 1

\begin{tabular}{|r||l||c|c||c|c||c|c||c|c||}
\hline \#unkn. & $h_{\max }$ & $\mathcal{E}_{K, 2}$ & EOC & $\mathcal{E}_{K, \infty}$ & EOC & $\mathcal{E}_{H, 2}$ & EOC & $\mathcal{E}_{H, \infty}$ & EOC \\
\hline \hline 2346 & 0.126 & 0.41 & $\cdot$ & 1.1 & $\cdot$ & 0.17 & $\cdot$ & 0.43 & $\cdot$ \\
\hline 9378 & 0.0717 & 0.41 & -0.015 & 1.2 & -0.19 & 0.18 & -0.014 & 0.47 & -0.13 \\
\hline 37506 & 0.0407 & 0.42 & -0.0048 & 1.2 & 0.088 & 0.18 & -0.0062 & 0.45 & 0.085 \\
\hline 150018 & 0.0231 & 0.42 & -0.0038 & 1.2 & 0.012 & 0.18 & -0.0041 & 0.44 & 0.012 \\
\hline 600066 & 0.0131 & 0.42 & -0.0022 & 1.2 & 0.0027 & 0.18 & -0.0023 & 0.44 & 0.0022 \\
\hline 2400258 & 0.00743 & 0.42 & -0.0012 & 1.2 & 0.0011 & 0.18 & -0.0012 & 0.48 & -0.13 \\
\hline
\end{tabular}

Polynomial degree: 2

\begin{tabular}{|r||l||c|c||l|l||l|l||l|l||}
\hline \#unkn. & $h_{\max }$ & $\mathcal{E}_{K, 2}$ & EOC & $\mathcal{E}_{K, \infty}$ & EOC & $\mathcal{E}_{H, 2}$ & EOC & $\mathcal{E}_{H, \infty}$ & EOC \\
\hline \hline 202 & 0.395 & 0.052 &. & 0.18 &. & 0.021 &. & 0.082 &. \\
\hline 802 & 0.242 & 0.025 & 1.5 & 0.13 & 0.74 & 0.0055 & 2.7 & 0.031 & 2. \\
\hline 3202 & 0.138 & 0.0073 & 2.2 & 0.048 & 1.7 & 0.0014 & 2.5 & 0.01 & 2. \\
\hline 12802 & 0.0784 & 0.0019 & 2.4 & 0.016 & 1.9 & 0.00034 & 2.4 & 0.0033 & 2. \\
\hline 51202 & 0.0441 & 0.00049 & 2.4 & 0.0053 & 2. & $8.6 e-05$ & 2.4 & 0.001 & 2. \\
\hline 204802 & 0.0249 & 0.00012 & 2.4 & 0.0017 & 2. & $2.2 e-05$ & 2.4 & 0.00033 & 2. \\
\hline 819202 & 0.014 & $3.1 e-05$ & 2.4 & 0.00053 & 2. & $5.4 e-06$ & 2.4 & 0.00011 & 2. \\
\hline 3276802 & 0.00787 & $7.7 e-06$ & 2.4 & 0.00017 & 2. & $1.4 e-06$ & 2.4 & $3.3 e-05$ & 2. \\
\hline
\end{tabular}

Polynomial degree: 3

\begin{tabular}{|r||l||l|l||l|l||l|l||l|l||}
\hline \#unkn. & $h_{\max }$ & $\mathcal{E}_{K, 2}$ & EOC & $\mathcal{E}_{K, \infty}$ & EOC & $\mathcal{E}_{H, 2}$ & EOC & $\mathcal{E}_{H, \infty}$ & EOC \\
\hline \hline 272 & 0.515 & 0.27 &. & 2.5 &. & 0.11 &. & 0.54 &. \\
\hline 1082 & 0.284 & 0.096 & 1.7 & 0.52 & 2.6 & 0.048 & 1.5 & 0.23 & 1.4 \\
\hline 4322 & 0.154 & 0.021 & 2.5 & 0.12 & 2.4 & 0.011 & 2.4 & 0.06 & 2.2 \\
\hline 17282 & 0.0863 & 0.0048 & 2.5 & 0.037 & 2. & 0.0028 & 2.4 & 0.02 & 1.9 \\
\hline 69122 & 0.0486 & 0.0012 & 2.4 & 0.01 & 2.2 & 0.0007 & 2.4 & 0.0061 & 2.1 \\
\hline 276482 & 0.0273 & 0.00031 & 2.4 & 0.0032 & 2. & 0.00018 & 2.4 & 0.0019 & 2. \\
\hline 1105922 & 0.0154 & $7.8 e-05$ & 2.4 & 0.001 & 2. & $4.5 e-05$ & 2.4 & 0.00061 & 2. \\
\hline 4423682 & 0.00856 & $2 e-05$. & 2.4 & 0.00034 & 1.9 & $1.1 e-05$ & 2.4 & 0.0002 & 1.9 \\
\hline
\end{tabular}

Polynomial degree: 4

\begin{tabular}{|r||l||c|c||l|c||c|c||l|c||}
\hline \#unkn. & $h_{\max }$ & $\mathcal{E}_{K, 2}$ & EOC & $\mathcal{E}_{K, \infty}$ & EOC & $\mathcal{E}_{H, 2}$ & EOC & $\mathcal{E}_{H, \infty}$ & EOC \\
\hline \hline 162 & 0.793 & 0.14 & $\cdot$ & 0.86 &. & 0.058 & $\cdot$ & 0.26 & $\cdot$ \\
\hline 642 & 0.448 & 0.025 & 2.9 & 0.14 & 3.2 & 0.012 & 2.7 & 0.056 & 2.7 \\
\hline 2562 & 0.244 & 0.0015 & 4.7 & 0.0081 & 4.7 & 0.0008 & 4.5 & 0.0039 & 4.4 \\
\hline 10242 & 0.144 & 0.00028 & 3.2 & 0.003 & 1.9 & 0.00014 & 3.4 & 0.0015 & 1.8 \\
\hline 40962 & 0.0817 & $1.9 e-05$ & 4.7 & 0.00026 & 4.3 & $9.7 e-06$ & 4.6 & 0.00014 & 4.2 \\
\hline 163842 & 0.0464 & $2.8 e-06$ & 3.4 & $7.5 e-05$ & 2.2 & $1.4 e-06$ & 3.4 & $3.8 e-05$ & 2.3 \\
\hline 655362 & 0.0261 & $2.4 e-07$ & 4.2 & $1.5 e-05$ & 2.8 & $1.2 e-07$ & 4.2 & $7.5 e-06$ & 2.8 \\
\hline 2621442 & 0.0146 & $3.4 e-08$ & 3.4 & $5.4 e-06$ & 1.8 & $1.7 e-08$ & 3.4 & $2.7 e-06$ & 1.8 \\
\hline
\end{tabular}




\subsection{Local refinement and coarsening}

ALBERT implements local mesh-refinement and coarsening by the newest-vertex bisection method as described in detail in [SCHMidT and SieBert 2000]. At the beginning each element $T$ of a shape-regular conforming triangulation is assigned a dedicated refinement edge. When an element is bisected, the refinement edges of its children are the edges opposite the newest vertex, see Figure 3.2. Algorithm 3.3.1 sketches the algorithm which removes non-conforming nodes in the sense of Definition 2.4.2 on page 43 above. [B $\ddot{A N S C H} 1991]$ shows that in the case of a fixed triangulated domain the newest-vertex bisection method guarantees the shape-regularity and conformance of the refined meshes.

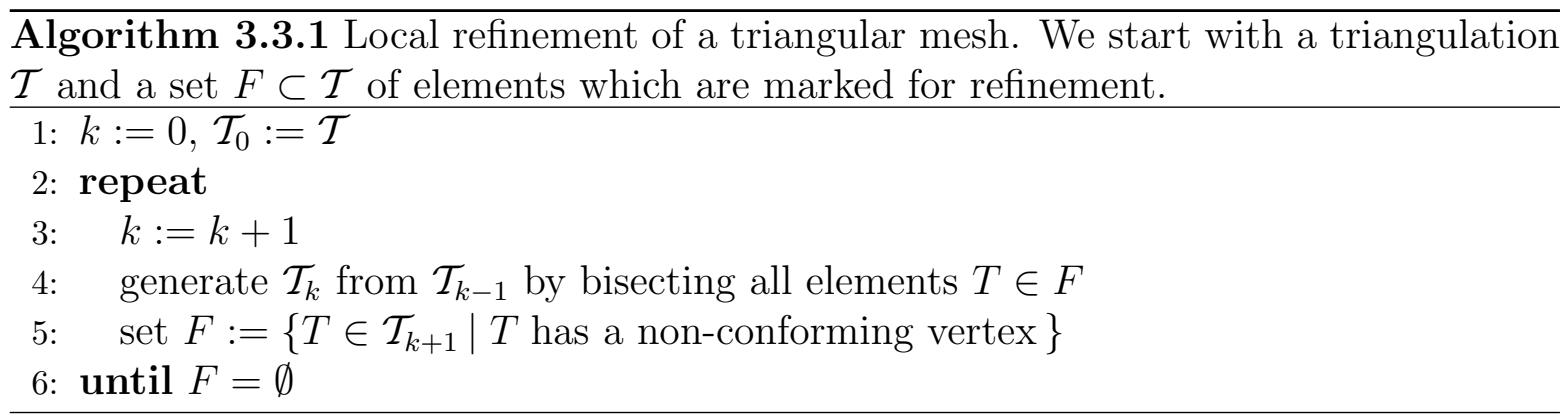

The case of an iso-parametric triangulation $\mathcal{T}_{h}^{d}$ can be handled similar to the case of planar triangulations described above. In the following we will use the notation introduced in Section 2.4.1. In the linearly parametrised case $d=1$ there is actually no difference.

If $d \geq 2$ then the only difference to the planar case is the following: the assignment of coordinates to the newly introduced nodes is more difficult. The coordinates are chosen such that the coordinates of the new nodes interpolate the old surface $\Gamma_{h}^{d}$. A natural choice is to map the Lagrange-nodes of the two child-elements of the appropriately bisected reference element $\hat{T}$ to the bisected iso-parametric element $T$ by means of the local parametrisation $\Phi_{T}^{d}$. Figure 3.3 on the following page shows an example for the case of $d=n=2$.

Figure 3.2 Refinement and coarsening of a conforming patch. The refinement edges are indicated by the dashed red lines. The blue dotted lines in the patch on the right mark the edges which would be inserted in the next refinement step.

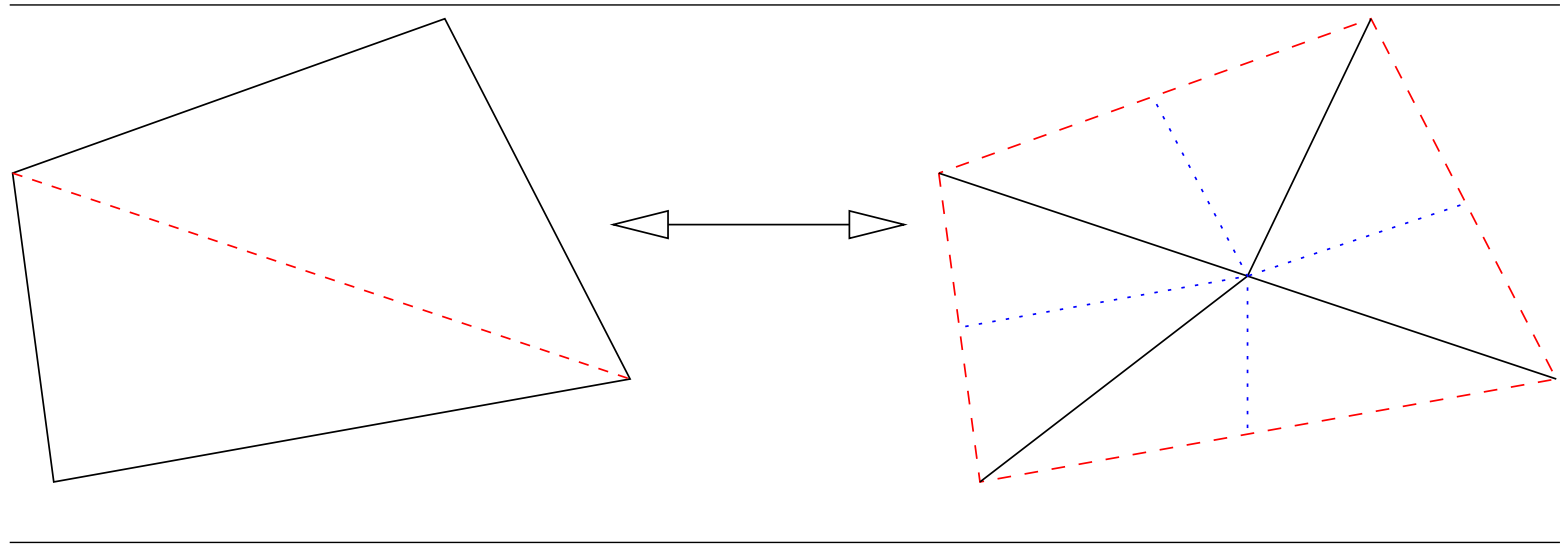


$\overline{\text { Figure 3.3 Refinement of an iso-parametric element of degree 2. The coordinates of }}$ the new nodes are the images under $\Phi_{T}^{2}$ of the childrenof the refined reference element. The new edge which bisects the reference element $\hat{T}$ and the iso-parametric element is shown in red, as well as the new nodes.

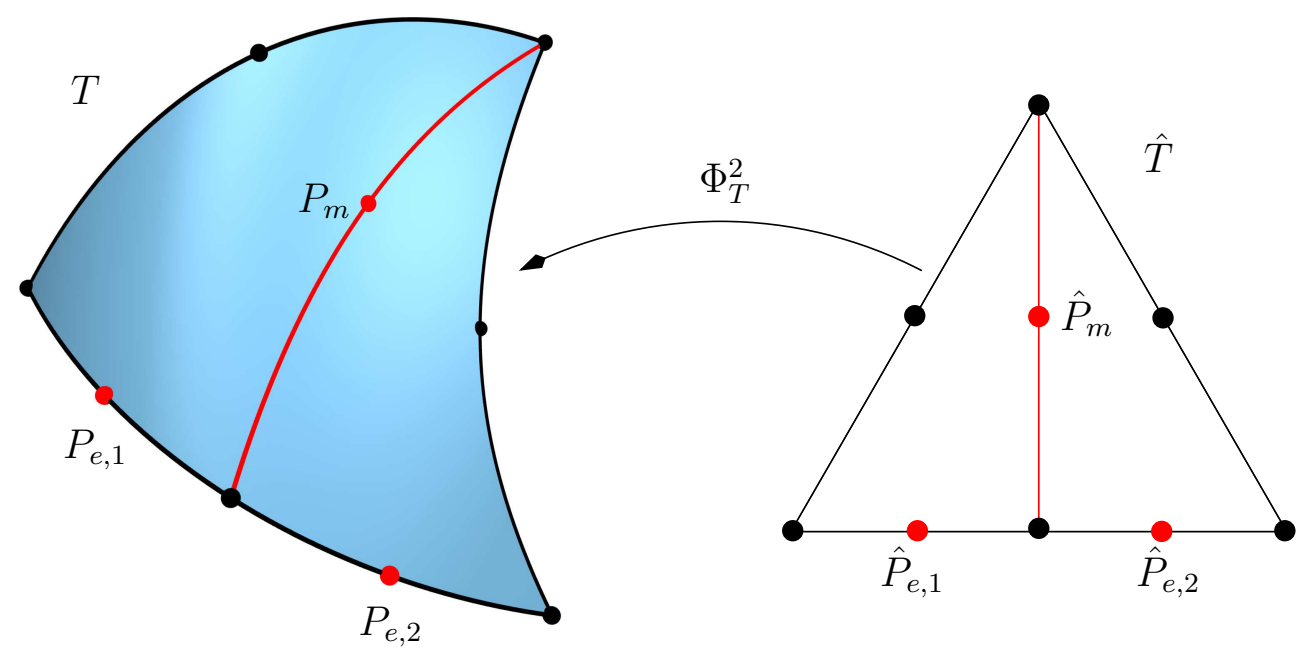

Because the identity mapping $\operatorname{id}_{\Gamma_{h}^{d}}$ of the iso-parametric discrete surface $\Gamma_{h}^{d}$ is itself contained in the iso-parametric finite element space $W_{h}^{d}\left(\Gamma_{h}^{d}\right)$ this choice for the new coordinates during the refinement of an iso-parametric element is just the usual interpolation step which maps a finite element function from the coarse mesh to the refined mesh.

Finally we remark that during the construction of an interpolation $\Gamma_{h}^{d}$ of a given smooth immersed (hyper-)surface $\Gamma$ we have to project the new nodes to the smooth surface $\Gamma$. We do this by first performing the interpolation step described above and afterwards use the lift in approximate normal direction with respect to the discrete surface to project the new coordinates to $\Gamma$.

\subsection{Mesh smoothing}

During the path-tracking algorithm for the drop shapes the surface mesh used in the discretisation of the problem may degenerate and develop very acute and very obtuse angles (see Figure 3.4(b) on the next page). Therefore it is a crucial point of the algorithm to counterbalance this degeneration by mesh-smoothing techniques, which eliminates this problem as shown in Figure 3.4(c) on the facing page.

\subsubsection{Subdivision of obtuse angles}

ALBERT uses the "newest vertex" algorithm (see Section 3.3) for mesh-adaption. This guarantees a stable triangulation in the planar case ([B̈̈NSCH 1991]). 


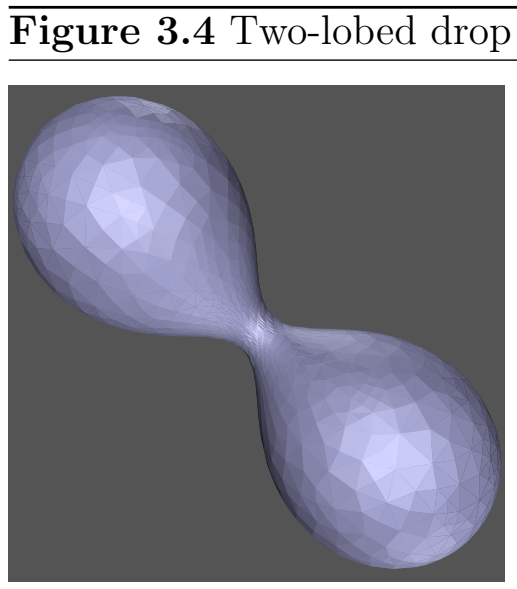

(a) Two-lobed drop, $\omega \simeq 0.208$, result of path-tracking without mesh-smoothing

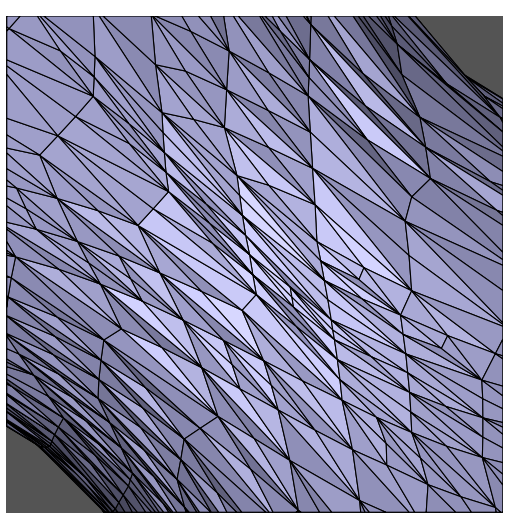

(b) Magnification of central region of (a)

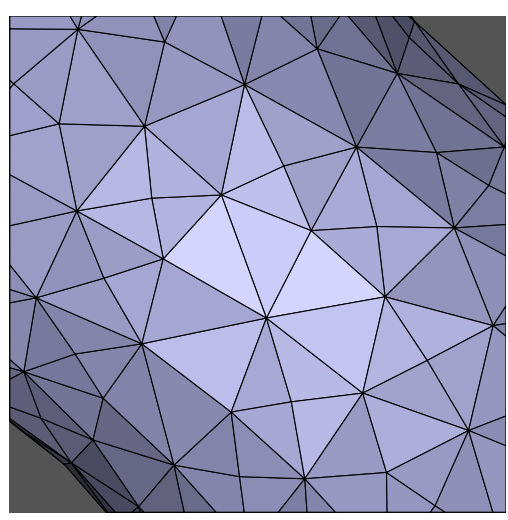

(c) Same value of $\omega$, created by path-tracking with meshsmoothing

However, as the surface mesh is subject to large deformations during the path-tracking algorithm, the stability of the triangulation is no longer guaranteed by this scheme. Therefore in [SCHMIDT 1993] - prior to the mesh-adaption step - the longest edge of each element is chosen as the refinement-edge, so that the largest angle will be divided when an element is refined. Subsequent bisections to resolve hanging nodes follow the newestvertex scheme (in contrast to [Mitchell 1989]), because this reduces the number of bisection steps needed to get a conformal triangulation again.

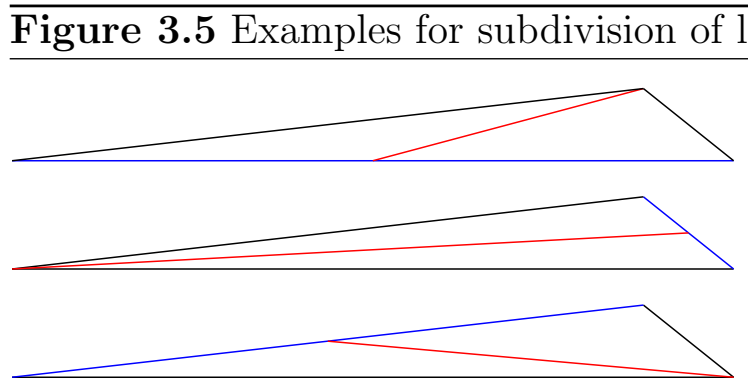

(a) 3 choices for the refinement edge (in blue). Angles of children need not be less obtuse than angles of the parent element.

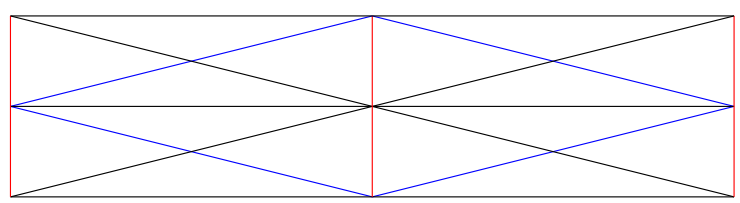

(c) Refine by red edges: acute angles cannot improve.

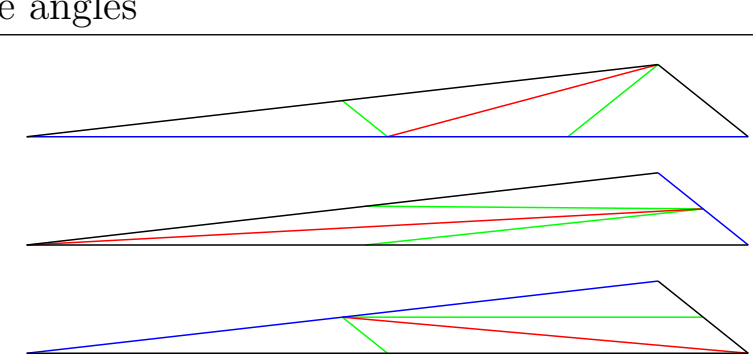

(b) Subsequent refinement steps (coloured). Topmost triangle: acute angles less sharp.

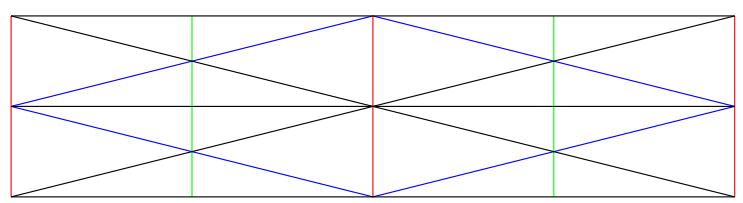

(d) Flip-flop effect: which each step alternately either obtuse or right angles occur.

We note that there is no guarantee that the children of a bisected element have significantly less obtuse angles. Nevertheless, choosing the largest edge as refinement edge might make things less worse. This is shown in Figure 3.5(b).

In addition to just reassigning the refinement edges [SCHMIDT 1993] also actively refines 
obtuse angles greater than a certain limit, $\frac{2 \pi}{3}$ being the limit of choice.

We adopt his techniques. However, we want to avoid the flip-flop effect illustrated in Figure 3.5(d) on the preceding page: there, when bisecting an element only once in each step of our path-tracking algorithm (or in a time-step for time dependent problems), obtuse and right angles alternate from each refinement step to the next.

Also, we do not always reassign the refinement edges to be opposite the largest angle in each element, but only when one of the interior angles indeed is obtuse, that is, larger than $\frac{\pi}{2}$. This way, our algorithm benefits from the robustness of the newest-vertex scheme locally, in regions where the triangulation is highly regular. It doesn't make sense to reassign the refinement edges in nearly equilateral triangles.

So, with regard to the division of obtuse angles, we arrive at Algorithm 3.4.1.

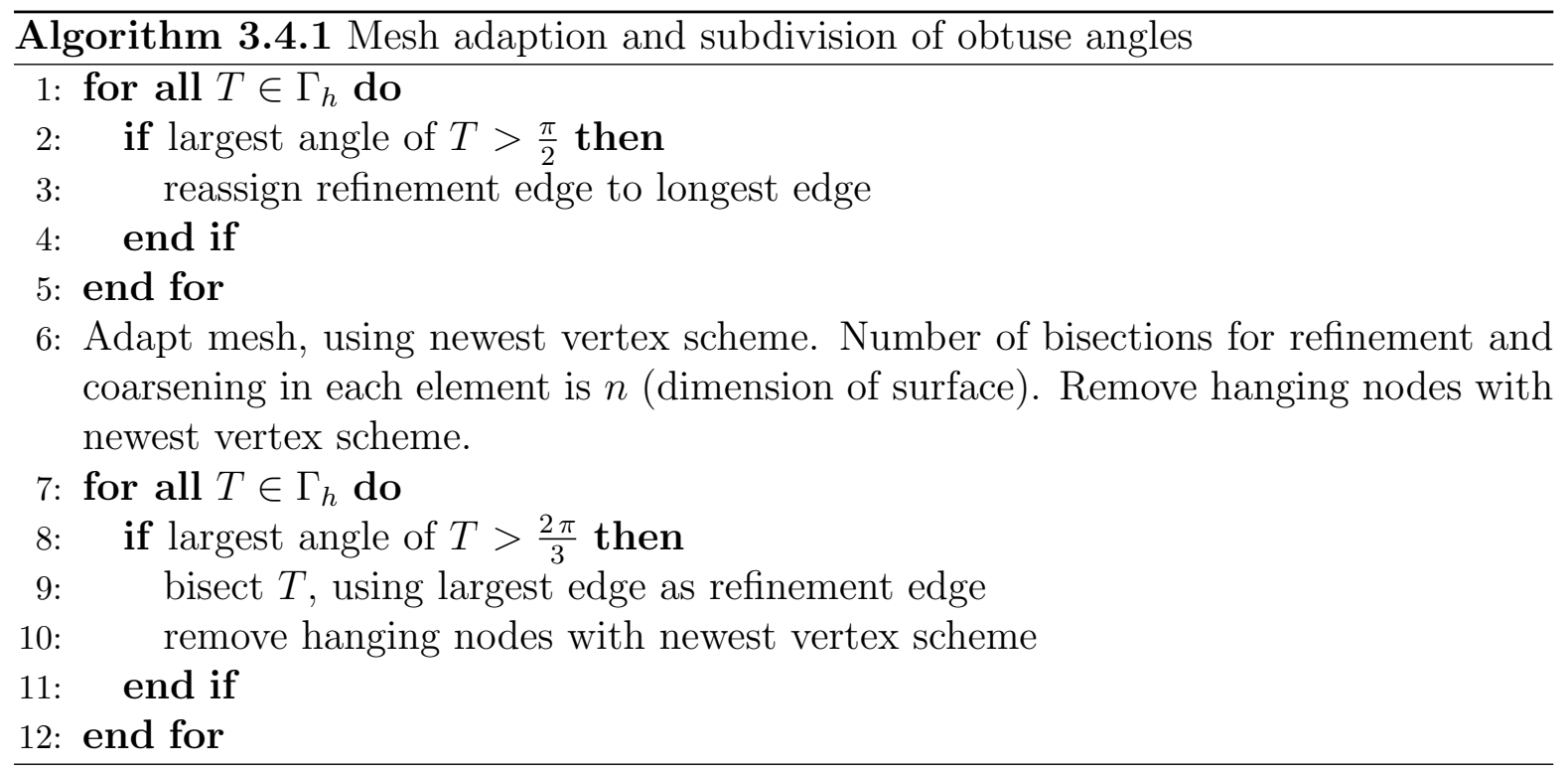

\subsubsection{Vertex displacement}

The mesh-smoothing method described in the previous section tries to improve the mesh quality by refinement techniques. This changes the topology of the triangulation but leaves already present vertices in place. It is not possible with this method to counterbalance the development of a distinct anisotropy in the node-density during the path-tracking algorithm, as indicated in Figure 3.5(d) on the preceding page.

Another possibility to improve the mesh quality is to leave the topological structure of the mesh unchanged and instead change the coordinates of the vertices to improve the triangulation, which is the subject of the subsequent sections.

While there are also "direct" methods available, which compute optimised coordinates for all vertices simultaneously - see e.g. [BALENDRAN 1999] for a very brief outline we have chosen a single-step method.

In the single-step setup, we loop over all vertices of the mesh and displace the vertices, sequentially, based on the shape of the "star" $S(P)$ around each vertex $P$, the union of 


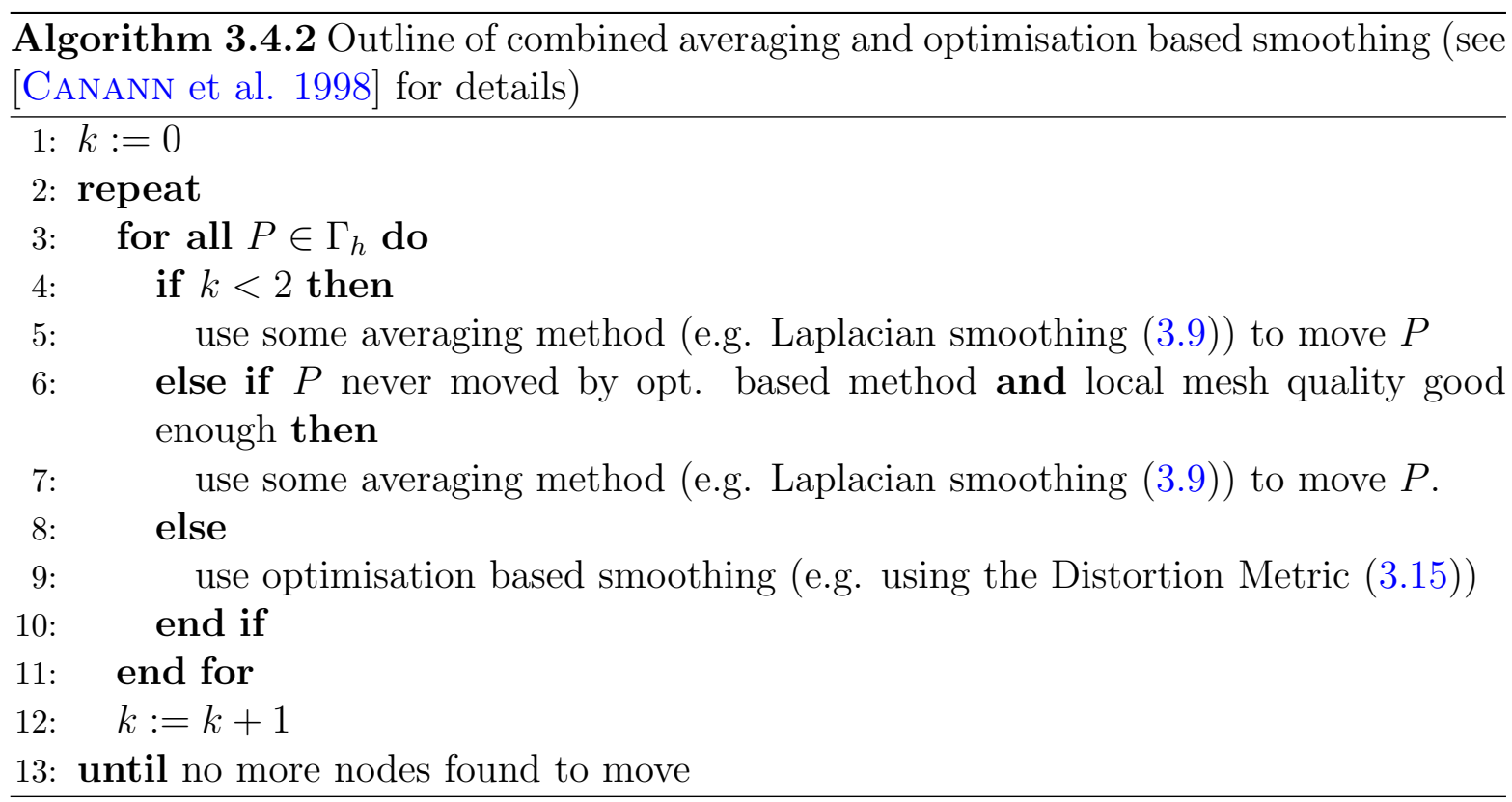

all triangles adjacent to $P$. This "main-loop" is then repeated until the mesh reaches a satisfactory state.

We expect - without further investigation - that a single-step method is more stable in the case of parametric meshes, because the displacement of each vertex will be small even for highly distorted meshes, and so it is easier to project the displaced nodes back to the surface.

Some existing single-step approaches In the literature there is the notion of "averaging methods", where the new position $A(P)$ of a node $P$ is computed directly from the data of its element neighbourhood $S(P)$, using an explicit mapping $A: S(P) \rightarrow \mathbb{R}^{m}$ where $m$ is the dimension of the embedding space. Two examples are "Laplacian smoothing" (3.9) - the new position of $P$ is the average of the end points of the edges adjacent to $P$ - and averaging about certain interior angles, as in (3.12) on page 79.

In contrast to this, one finds the notion of "optimisation based" methods, which compute the new location by minimising a functional $F$ defined on the element-neighbourhood $S(P)$ of $P$. Actually, the most commonly used averaging methods are just special cases of optimisation based methods where the minimiser $P_{\min }$ of $F$ can be computed by a closed, comparatively simple formula, see (3.8) and (3.10) on page 78.

Algorithm 3.4.2 gives a very short outline of a combined method, as described in [CANANN et al. 1998]. It uses computational cheap averaging methods in regions where the local mesh quality is good enough, and invokes optimisation based smoothing only for sufficiently ill-shaped elements. This technique aims at reducing the computational cost of an optimisation-based smoothing method considerably by still retaining a highly regular mesh.

For optimisation based methods, the simplest way to decrease the value of the smoothingfunctional $F$ locally is a steepest descent method in the direction opposite to the gradient of $F$. 
In contrast to this method [DJIDJEV 2000] proposes to use the search direction of the previous iteration-step of the main-loop of his single-step algorithm for the update of a new search direction in a conjugate-gradient manner (see Algorithm 3.4.3 on the facing page). Unluckily in the examples we have tried this leads to a non-stable smoothing-algorithm, in the sense that the conjugate-gradient method (CG-method) Algorithm 3.4.3 on the next page converges more slowly than the more simpler steepest decent method.

The problem might be that the gradient of the smoothing-functional $F$ at $P$ can be altered a lot when a neighbouring node is moved. Therefore, the gradients and CGdirections of the previous iteration might not contain enough information about the current state.

It has to be noted, though, that in the single-step approach a lot of parameters come into play which influence the smoothing-algorithm: for example the maximum distance to move a node inside its patch $S(P)$, the accuracy of the computation of the local minimiser (good local accuracy does not imply good global quality!), the order in which the nodes are displaced. Those parameters do not only influence the efficiency of the mesh-smoothing, but also the final state. Thus the reason that we could not use the CG-style method presented in [DJIDJEV 2000] simply might be that our parameters were not suitable for doing so.

Single-step method for parametric meshes For the moment we are only concerned with piecewise linearly parametrised surface meshes. A simple extension to local parametrisations of higher polynomial degree is given at the end of this paragraph.

We do not use the combined Algorithm 3.4.2 on the page before: as illustrated below in Figure 3.10 on page 85 , the global redistribution of the vertices caused by the averaging methods (3.9) and (3.12) is significantly different from the redistribution caused by the most powerful smoothing-functionals (3.15) and (3.16). (3.15) and (3.16) tend to increase the node-density in regions of high curvature (where a highly refined mesh is needed), but (3.9) and (3.12) do not.

Therefore we use a pure optimisation-based approach, but avoid unnecessary work by only moving nodes where the smoothing-functional has a large gradient and so the expected improvement of the local mesh quality is high enough.

Regardless of whether using a direct or a single-step method, it is an important constraint for the displacement of the nodes that they must remain on the surface. As a global parametrisation of the discrete surface is not available, it would be hard to incorporate this constraint directly into the computation of the vertex displacements. Instead, one first computes the optimised node positions ignoring the on-surface constraint. Afterwards, the displaced nodes are projected back to the original surface. The projection is a crucial and non-trivial step of the smoothing algorithm and is therefore explained in detail in Section 3.4.4 on page 88 below.

This way the computation of the new coordinates is simplified, but the smoothed mesh still interpolates the discrete surface $\Gamma_{h}$, and the additional error introduced by meshsmoothing is a mere interpolation error. The problem of projecting a point to the discrete surface $\Gamma_{h}$ is addressed in Section 3.4.4. The general approach for a single-step 


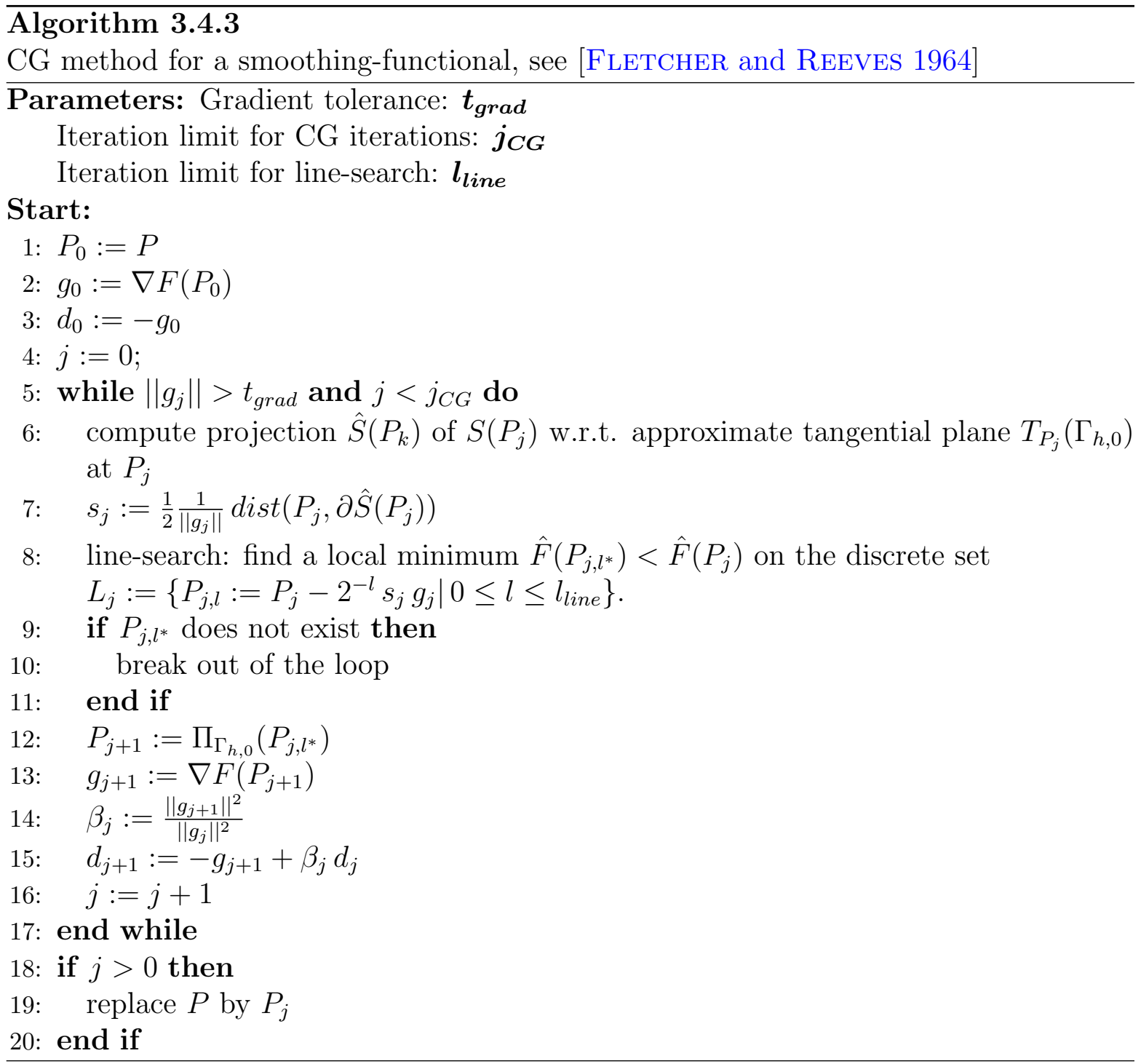

algorithm for parametric meshes is to remember the original surface $\Gamma_{h, 0}:=\Gamma_{h}$ at the start of the single-step algorithm. This involves only storage of the coordinates of the nodes of the original mesh as the topological structure of the mesh does not change during the smoothing process. Then we are able to iterate the single-step smoothing method and produce a sequence $\Gamma_{h, k}, k=1,2, \ldots$ of triangulations, where in each step the stored coordinates of the original surface are used to compute the projection back to $\Gamma_{h, 0} \equiv \Gamma_{h}$. In the following we denote this projection by $\Pi_{\Gamma_{h, 0}}$ ([SCHMIDT 1993]).

In our implementation, we first project the element-neighbourhood $S(P)$ of a vertex $P \in \Gamma_{h, k}$ to an approximate tangential (with respect to $\Gamma_{h, 0}$ ) plane at $P$. Then we compute the new position $\hat{P}$ of $P$ with respect to the projected star $\hat{S}(P)$. Compared to allowing arbitrary displacements in $\mathbb{R}^{3}$ this stabilises the node-displacement with respect to the projection $\Pi_{\Gamma_{h, 0}}$ in regions of high curvature, because the displaced node $\hat{P}$ remains closer to the original surface. Especially this approach inhibits displacements which only lead off the surface and would be annihilated by the subsequent projection step. 


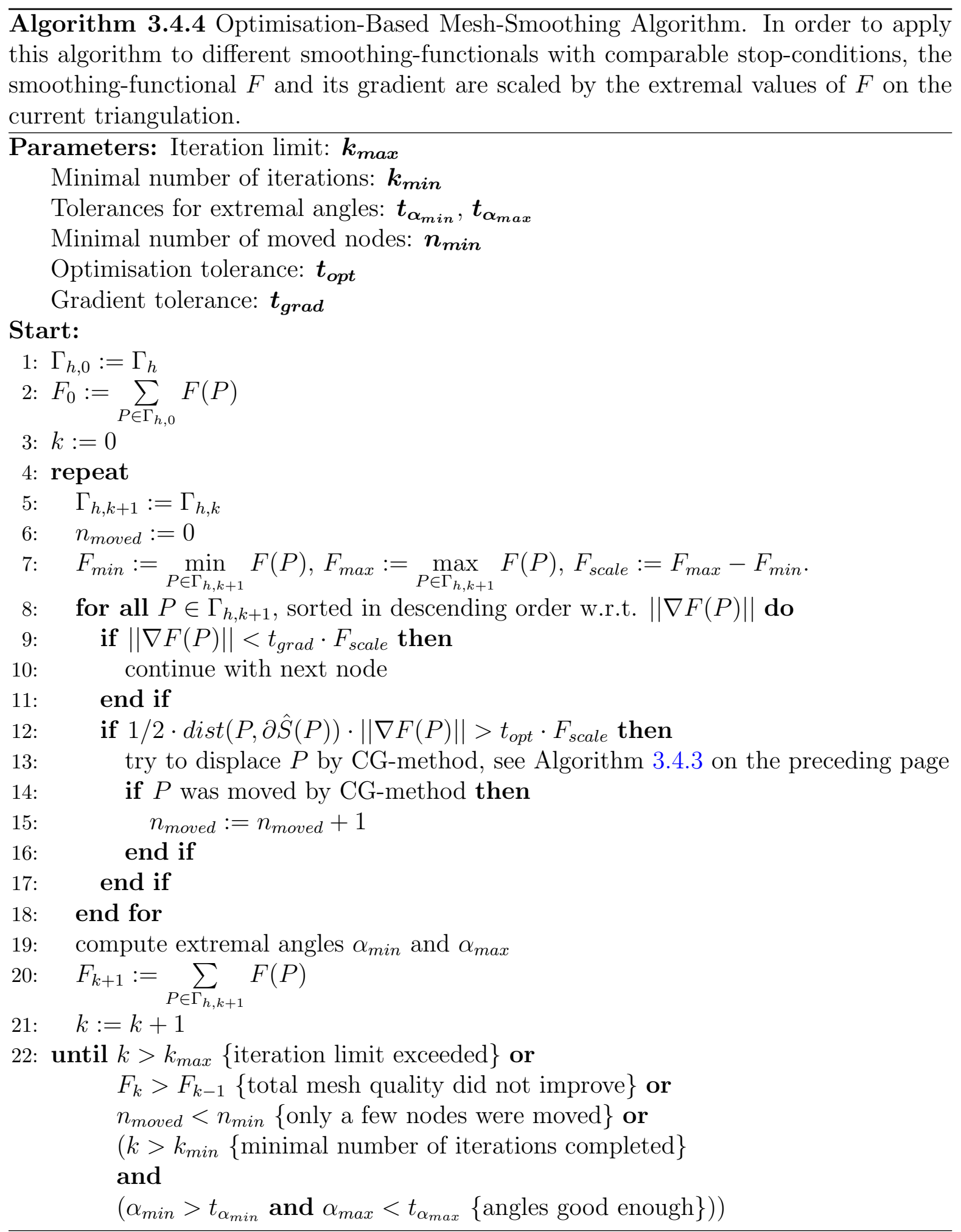


In order to find good new position $\hat{P}$ for $P$ inside the projected element neighbourhood $\hat{S}(P)$ - such that $F(\hat{P})$ is close to minimal - a few steps (typically 2 or 3 ) of a nonlinear conjugate gradient method are applied (see Algorithm 3.4.3 on page 75). This was inspired by [DJIDJEV 2000], though we were not able to copy his method, see the remarks on page 74 .

The main part of our optimisation-based smoothing algorithm is described in $\mathrm{Al}$ gorithm 3.4.4 on the facing page. Suitable choices for the optimisation functional $F$ are investigated in Section 3.4.3 below.

Parametrisations of higher polynomial degree So far our approach only covers piecewise linearly-parametrised surfaces. As described in [SCHMIDT 1993] for a surface $\Gamma_{h}$ which's elements are parametrised with Lagrange-polynomials of degree 2 or greater the smoothing algorithm acts only on the vertex-nodes of the discretised surface $\Gamma_{h}$ : the vertex nodes constitute a triangulated surface $\Gamma_{h}^{l i n}$ which is then smoothed as described above, with the difference that the projection $\Pi_{\Gamma_{h, 0}}$, of course, uses the non-linear parametrisation of $\Gamma_{h}$.

After the smoothing-algorithm has terminated, producing a smoothed triangular surface mesh $\Gamma_{h, k}^{l i n}$, the none-vertex Lagrange-nodes of each element with respect to the chosen polynomial parametrisation are projected by $\Pi_{\Gamma_{h, 0}}$ to $\Gamma_{h}$, hereby generating the nonlinearly parametrised smoothed mesh.

For the parametrisation with quadratic Lagrange-polynomials this means that the midpoints of the edges of $\Gamma_{h, k}^{l i n}$ are projected to $\Gamma_{h, 0}$ and constitute the non-vertex nodes of the final mesh.

\subsubsection{Smoothing functionals}

For evaluating the local mesh-quality we use a functional $F(P)$ defined on the star $S(P)$ around a vertex $P$ of the triangulation.

To fix the notation (see the figure on the right) let $N$ denote the number of the triangles $T_{i}$ around $P, 1 \leq i \leq N$, so $S(P)=$ $\bigcup_{i=1}^{N} T_{i}$.

For $1 \leq i \leq N$ let $Q_{i}, 1 \leq i \leq$ $N, Q_{N+1}:=Q_{1}$, be the vertices of $S$ such that $Q_{i}, Q_{i+1}$ and $P$ are the vertices of $T_{i}$. Further let $v_{i}:=Q_{i}-P$, with $v_{N+1}:=v_{1}$, denote the edges adjacent to $P$ and $w_{i}:=Q_{i+1}-Q_{i}=v_{i+1}-v_{i}$, $w_{N+1}:=w_{1}$, the edges opposite to $P$.

The non-unit element normals are denoted by $\nu_{i}:=v_{i} \wedge v_{i+1}(1 \leq i \leq N)$. The normals $n u_{i}$ have been omitted from the figure on the right.

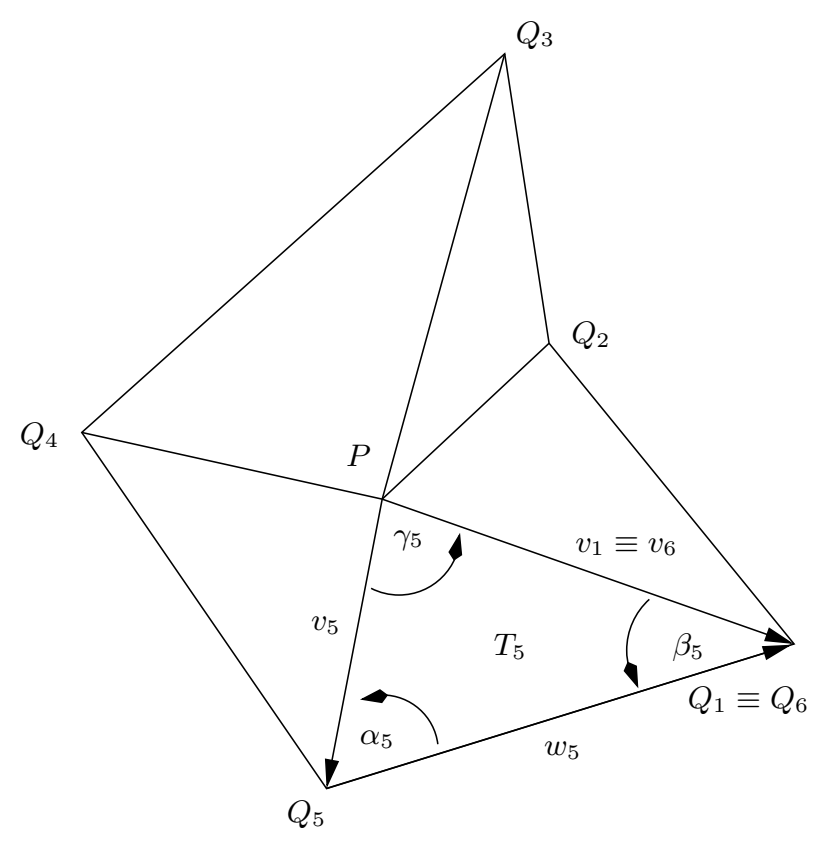


Finally $\alpha_{i}:=\angle\left(w_{i},-v_{i}\right), \beta_{i}:=\angle\left(v_{i+1}, w_{i}\right)$ and $\gamma_{i}:=\angle\left(v_{i}, v_{i+1}\right)$ denote the interior angles of $T_{i}, 1 \leq i \leq N$ as shown in the drawing above.

In the case of a parametric surface $\Gamma_{h}$, we actually do not use $S(P)$, but its projection $\hat{S}(P)$ to an approximate tangential plane at $P$. However, to simplify notation we will not distinguish between $S(P)$ and $\hat{S}(P)$ in the following, and assume that $S(P)$ is planar, but embedded into $\mathbb{R}^{3}$.

For the derivation of the formulas for the gradients of the smoothing-functionals it has to be noted that $P$ is the independent variable and

$$
\nabla\left\|\nu_{i}(P)\right\|=\frac{\nu_{i}(P)}{\left\|\nu_{i}(P)\right\|} \wedge w_{i}
$$

This is clear because $\left\|\nu_{i}\right\|$ is the volume of the parallelogram spanned by $w_{i}$ and $v_{i}$. So the steepest-ascent direction clearly is orthogonal to $w_{i}$, moving $P$ away from $w_{i}$, and the amount of increase is proportional to $\left\|w_{i}\right\|$ (which is independent from $P$ ).

There are several possible choices for $F$. In his survey about unstructured mesh generation [OwEN 1998] gives a brief summary and large list of references. In our work we have considered and implemented the following smoothing-functionals:

Discrete Laplacian The length of the edges adjacent to $P$ is minimised:

$$
\begin{gathered}
F_{\Delta}(P):=\sum_{i=1}^{N}\left\|v_{i}\right\|^{2}, \\
\nabla F_{\Delta}(P)=-2 \sum_{i=1}^{N} v_{i} .
\end{gathered}
$$

The exact position of the minimum of $F_{\Delta}$ can be computed easily, it is just the mean value of the vertices $Q_{i}, 1 \leq i \leq N$. This method is the probably most often used averaging smooth-method. The new position of $P$ is given by

$$
A_{\Delta}(P):=\frac{1}{N} \sum_{i=1}^{N} Q_{i} .
$$

$F_{\Delta}$ can be viewed as the energy of a mechanical system where the edges adjacent to $P$ are replaced by linear springs. This was probably the motivation for the following averaging method.

Torsion Springs In [ZHou and Shimada 2000] the energy of a torsion-spring system is used:

$$
F_{\text {tors }}(P):=\sum_{i=1}^{N}\left(\alpha_{i}^{2}+\beta_{i}^{2}\right) .
$$

The authors define an iterative process, which decreases $F_{\text {tors }}$ : rotate the interior edges $v_{i}$ of $S(P)$ around their starting points $Q_{i}$ until the edges half the angles 
at $Q_{i}$. The new position for $P$ is the average of the coordinates of the end-points of the rotated edges. This can be used to define an iteration which finally yields a minimiser for $F_{\text {tors }}$ by a limiting process. For convenience let $\bar{\nu},\|\bar{\nu}\|=1$, be an approximate normal at $P$ (just $(0,0,1)$ in the 2 -dimensional case). Then let $P_{\text {tors }, 0}:=P$ and for $k \in \mathbb{N}_{0}$ define

$$
P_{\text {tors }, k+1}:=\frac{1}{N} \sum_{i=1}^{N}\left(\left\|v_{i+1}\left(P_{\text {tors }, k}\right)\right\|\left(\bar{\nu} \wedge \frac{\frac{w_{i+1}}{\left\|w_{i+1}\right\|}+\frac{w_{i}}{\left\|w_{i}\right\|}}{\left\|\frac{w_{i+1}}{\left\|w_{i+1}\right\|}+\frac{w_{i} \|}{\left\|w_{i}\right\|}\right\|}\right)+Q_{i+1}\right) .
$$

Compared with the computations described by [ZHOU and SHIMADA 2000] this formula avoids trigonometric functions at the cost of additional square-roots.

The exact position of the minimiser can be obtained by the limit

$$
A_{\text {tors }}(P):=\lim _{k \rightarrow \infty} P_{\text {tors }, k} .
$$

We did not investigate whether there exists an explicit formula for the minimiser, though this would be an interesting point for the application of (3.10) as smoothing-functional.

The authors do not use (3.12), but apply in each step of the smoothing algorithm only one iteration step of (3.11).

\section{Conformal Energy}

[SchmidT 1993] uses the "conformal energy" $E_{C}$ of [Hutchinson 1991],

$$
E_{C}(x):=\int_{D_{N}}\|\nabla x\|^{2}-2 \int_{D_{N}}\left\|x_{u} \wedge x_{v}\right\|
$$

where $D_{N}$ is the regular $N$-polygon inscribed into the unit-circle and $x: D_{N} \rightarrow$ $S(P):(u, v) \mapsto x(u, v)$ denotes a parametrisation of $S(P)$ over $D_{N}$. It holds that $E_{C}(x) \geq 0$ and $E_{C}(x)=0$ if and only if $x$ is a conformal parametrisation.

In the discrete case one restricts oneself to (continuous!) parametrisations which map the facets of $D_{N}$ linearly to the facets of $S(P)$ (so vertices are mapped to vertices and the central of $D_{N}$ is mapped to $P$ ). This yields the following formula for the "conformal energy" $E_{C}(P)$ of $S(P)$, where $\alpha:=\frac{2 \pi}{N}$ :

$$
\begin{aligned}
F_{\text {con }}(P) & :=E_{C}(P)=\sum_{i=1}^{N}\left(\frac{\left\|v_{i}\right\|^{2}+\left\|v_{i+1}\right\|^{2}-2 \cos (\alpha)\left\langle v_{i}, v_{i+1}\right\rangle}{2 \sin (\alpha)}-\left\|\nu_{i}\right\|\right), \\
\nabla F_{\text {con }}(P) & =-\sum_{i=1}^{N}\left(\frac{1-\cos (\alpha)}{\sin \alpha}\left(v_{i}+v_{i+1}\right)+\frac{\nu_{i}}{\left\|\nu_{i}\right\|} \wedge w_{i}\right),
\end{aligned}
$$

with $\nu_{i}=v_{i} \wedge v_{i+1}$ (see the figure on page 77 for the notation). A detailed derivation of (3.13) can be found in [SCHMIDT 1993].

It has to be noted, however, that in the planar case $F_{c o n}$ is identical to $F_{\text {edge }}$, up to a factor and an additive constant, as long as the triangles are not inverted (see 
$\overline{\text { Figure 3.6 Contour plots of some smoothing-functionals. The position of the minimum }}$ is indicated by the central node. For the smoothing-functionals see equations (3.8)(3.16).

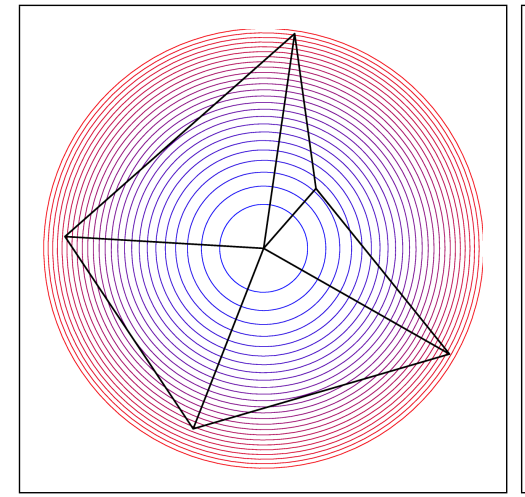

(a) $F_{\Delta}$, Laplacian

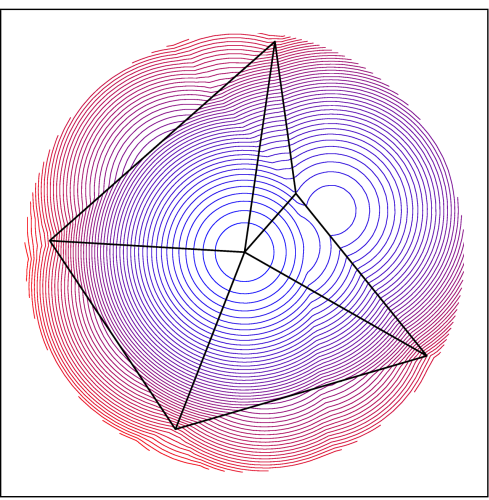

(b) $F_{c o n}$, conformal energy

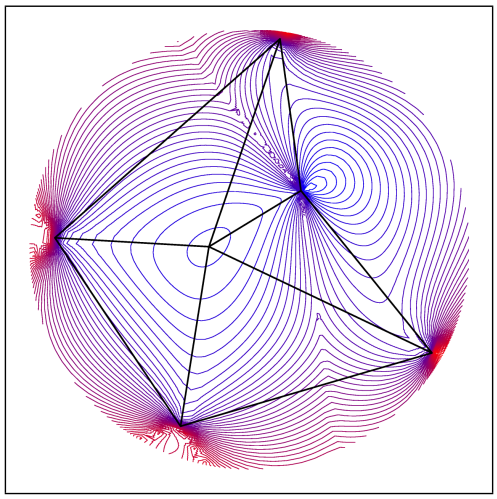

(c) $F_{\text {tors }}$, torsion springs

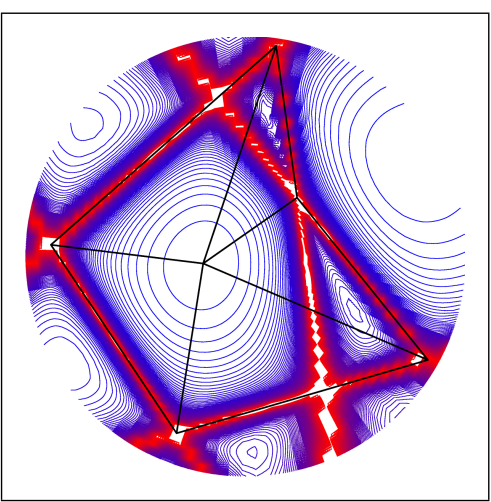

(d) $F_{\text {dist }}$, distortion metric

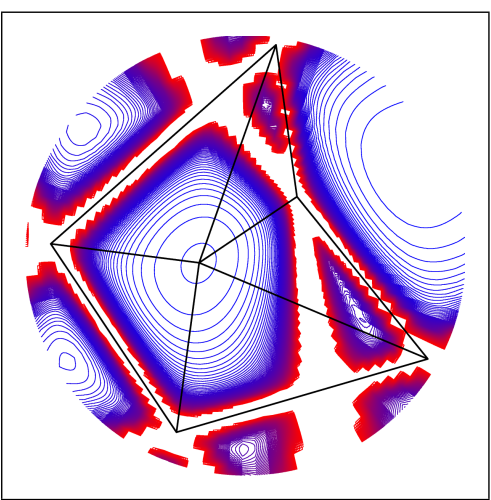

(e) $F_{\cot ^{2}}$, squared cotangens

Figure 3.6(a) and Figure 3.6(b)). This follows from:

$$
\begin{gathered}
\left\|v_{i+1}\right\|^{2}-2\left\langle v_{i+1}, v_{i}\right\rangle+\left\|v_{i}\right\|^{2}=\left\|v_{i+1}-v_{i}\right\|^{2}=\left\|Q_{i+1}-Q_{i}\right\|^{2}=\left\|w_{i}\right\|^{2} \\
\Longleftrightarrow-2 \cos (\alpha)\left\langle v_{i}, v_{i+1}\right\rangle=\cos (\alpha)\left(\left\|w_{i}\right\|^{2}-\left\|v_{i}\right\|^{2}-\left\|v_{i+1}\right\|^{2}\right) \quad(1 \leq i \leq N) .
\end{gathered}
$$

So we get

$$
\begin{aligned}
F_{\text {con }}(P) & =\sum_{i=1}^{N}\left(\frac{\left\|v_{i}\right\|^{2}+\left\|v_{i+1}\right\|^{2}-2 \cos (\alpha)\left\langle v_{i}, v_{i+1}\right\rangle}{2 \sin (\alpha)}-\left\|\nu_{i}\right\|\right) \\
& =\sum_{i=1}^{N}\left(\frac{1-\cos (\alpha)}{2 \sin (\alpha)}\left(\left\|v_{i}\right\|^{2}+\left\|v_{i+1}\right\|^{2}\right)+\frac{1}{2} \cot (\alpha)\left\|w_{i}\right\|^{2}\right)-2|S(P)| \\
& =\frac{1-\cos (\alpha)}{\sin (\alpha)} F_{\Delta}(P)+\frac{1}{2} \cot (\alpha) \sum_{i=1}^{N}\left\|w_{i}\right\|^{2}-2|S(P)|
\end{aligned}
$$

Neither the boundary edges $w_{i}$ nor the area $|S(P)|$ of $S(P)$ depend on the position of the central node $P$. In the parametric case, when $P$ is constrained to move on the 
Figure 3.7 Contour plots of some smoothing-functionals for a highly distorted patch. Edges leading to inverted triangles are shown in red.

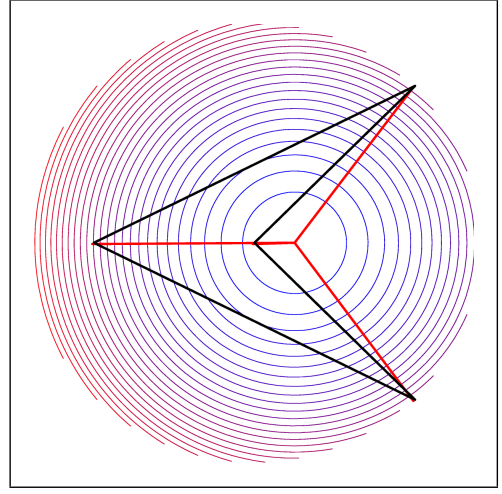

(a) $F_{\Delta}$, Laplacian

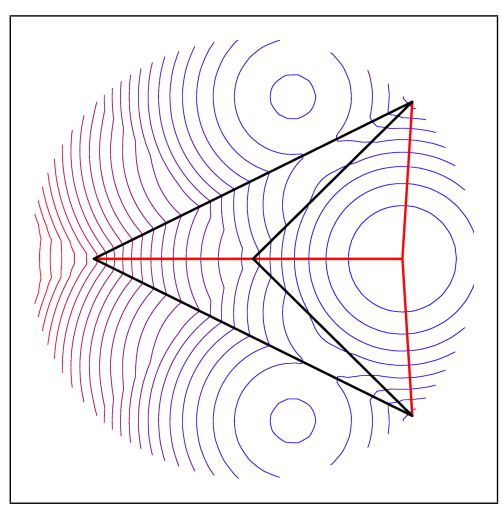

(b) $F_{c o n}$, conformal energy

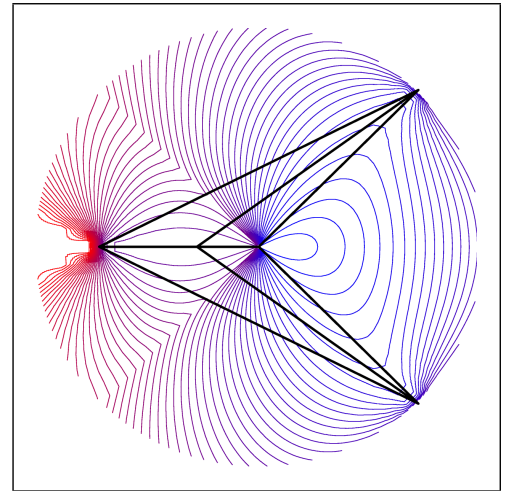

(c) $F_{\text {tors }}$, torsion springs

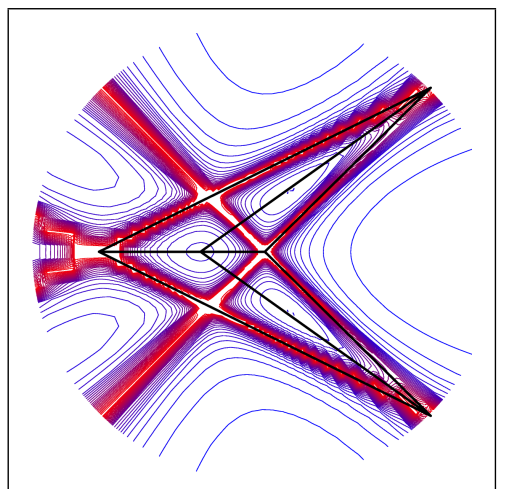

(d) $F_{\text {dist }}$, distortion metric

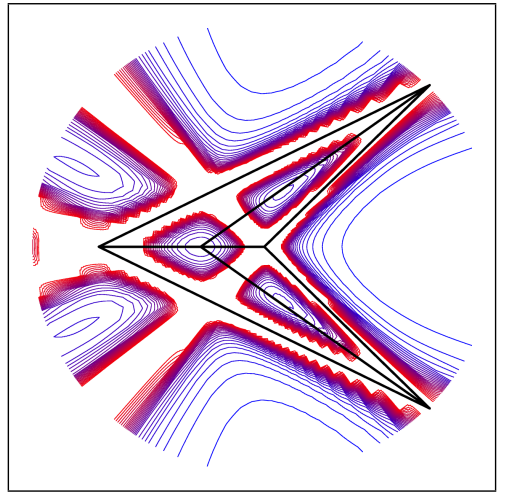

(e) $F_{\cot ^{2}}$, squared cotangens

surface $\Gamma_{h}, F_{\text {con }}$ penalises loss of area, which might explain why [SCHMIDT 1993] observed that in the parametric case this method is slightly better than mere Laplacian smoothing.

However, as we project $S(P)$ to an approximate tangent plane at $P$, our algorithm doesn't see this difference. The only remaining difference to Laplacian smoothing is the weight-factor $\frac{1-\cos (\alpha)}{\sin (\alpha)}$, which changes the order in which our optimisation based single-step mesh-smoothing Algorithm 3.4.4 on page 76 displaces the nodes.

Distortion Metric In [CANANn et al. 1998] a so called "distortion metric" is used. For any triangle $T_{i} \subset S(P)$ the quantity $\mathcal{D}\left(T_{i}\right)$ defined as

$$
\mathcal{D}\left(T_{i}\right):=\frac{\left\|v_{i}\right\|^{2}+\left\|v_{i+1}\right\|^{2}+\left\|w_{i}\right\|^{2}}{2\left\|\nu_{i}\right\|}
$$

is used as a measure the distortion of $T_{i}$ from the equilateral form. Up to a factor $\mathcal{D}\left(T_{i}\right)$ is the ratio of the sum of the squared edge lengths of $T_{i}$ to its area. It is 
minimal for equilateral triangles. The smoothing-functional for this case is

$$
\begin{aligned}
F_{\text {dist }}(P) & :=\sum_{i=1}^{N} \mathcal{D}\left(T_{i}\right)=\sum_{i=1}^{N} \frac{\left\|v_{i}\right\|^{2}+|| v_{i+1}||^{2}+|| w_{i} \|^{2}}{2 \| \nu_{i}||} \\
& =\sum_{i=1}^{N}\left(\left|\cot \alpha_{i}\right|+\left|\cot \beta_{i}\right|+\left|\cot \gamma_{i}\right|\right) \\
& =\sum_{i=1}^{N} \frac{\left|\left\langle v_{i}, v_{i+1}\right\rangle\right|+\left|\left\langle v_{i+1}, w_{i}\right\rangle\right|+\left|\left\langle v_{i}, w_{i}\right\rangle\right|}{\| \nu_{i}||}, \\
\nabla F_{\text {dist }}(P) & :=-\sum_{i=1}^{N}\left(\frac{v_{i}+v_{i+1}}{\| \nu_{i}||}+\frac{F_{\text {dist }}(P)}{2\left\|\nu_{i}\right\|^{2}} \nu_{i} \wedge w_{i}\right) .
\end{aligned}
$$

Squared Cotangens The following is a modification of (3.15); the sum is taken over the square of the cotangens of the interior angles:

$$
\begin{aligned}
F_{\cot ^{2}}(P) & :=\sum_{i=1}^{N}\left(\cot ^{2}\left(\alpha_{i}\right)+\cot ^{2}\left(\beta_{i}\right)+\cot ^{2}\left(\gamma_{i}\right)\right) \\
& =\sum_{i=1}^{N} \frac{\left\langle v_{i}, v_{i+1}\right\rangle^{2}+\left\langle v_{i+1}, w_{i}\right\rangle^{2}+\left\langle v_{i}, w_{i}\right\rangle^{2}}{\left\|\nu_{i}\right\|^{2}}, \\
\nabla F_{\cot ^{2}}(P) & =-2 \sum_{i=1}^{N} \frac{\left\langle v_{i}, v_{i+1}\right\rangle\left(v_{i}+v_{i+1}\right)+\left\langle v_{i}+v_{i+1}, w_{i}\right\rangle w_{i}+F_{\cot ^{2}}(P) \nu_{i} \wedge w_{i}}{\left\|\nu_{i}\right\|^{2}} .
\end{aligned}
$$

Contour-plots of the smoothing-functionals (3.8)-(3.16) are shown in Figure 3.6 on page 80 . In compliance with $(3.14), F_{\Delta}$ and $F_{\text {con }}$ have the same level-sets inside the region where the triangles of the patch are not inverted. Figure 3.6(d) and 3.6(e) on page 80 illustrate that $F_{\text {dist }}$ and $F_{\cot ^{2}}$ diverge when the central nodes approaches a position which leads to degenerated triangles. This has a stabilising effect for our single step Algorithm 3.4.4 on page 76, because triangles become less easily inverted.

In contrast to this, $F_{\Delta}, F_{\text {tors }}$ and $F_{\text {con }}$ are continuous across "degeneration"-lines, so that Algorithm 3.4.4 on page 76 additionally has to check for inverted triangles. As illustrated in Figure 3.7 on the page before, there are even cases where the minimiser for $F_{\Delta}$ (and also for $F_{c o n}$ ) is located outside the patch $S(P)$ around $P$.

Mesh-smoothing for a planar test-domain As a first test for the behaviour of the smoothing-functionals listed above, we apply the two averaging methods (3.9) and (3.11) and the optimisation-based Algorithm 3.4.4 on page 76 with the smoothing-functionals listed above to the planar triangulation shown in Figure 3.8 on the next page.

This figure was created by deforming a globally refined rectangular domain. The colouring measures the quality of the elements in terms of the minimum and maximum angles (blue is better). The overall minimum and maximum angles were 6.86 and 155.39 degrees. 
The mesh-smoothing was carried out until Algorithm 3.4.4 on page 76 terminated because it couldn't find any better node-position up to machine-precision.

The final angles - see Table 3.5 - show only very little difference between explicit Laplacian smoothing, optimisation based Laplacian smoothing and optimisation based smoothing with the conformal energy $F_{\text {con }}$ (Figures 3.9(a) to 3.9(d) on the following page).

This was, of course, to be expected, as this only difference between $F_{\Delta}$ and $F_{c o n}$ is the weight factor $\frac{1-\cos (\alpha)}{\sin (\alpha)}$, which compensates for the number of edges adjacent to each node (see equation (3.14) on page 80).

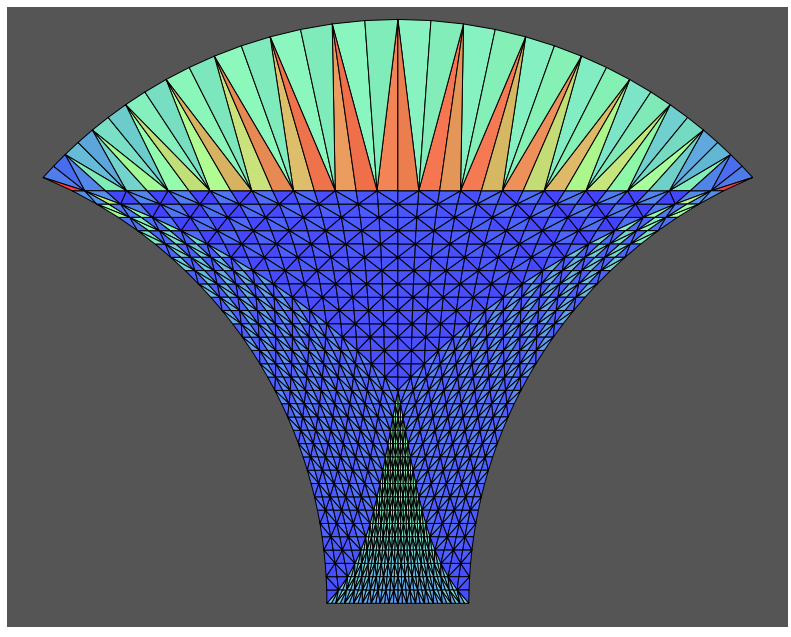

Figure 3.8 Planar test triangulation

Table 3.5 Extremal angles for the triangulated domain from Figure 3.9 on the following page, after mesh-smoothing

\begin{tabular}{|l||l|c|}
\hline & minimum angle & maximum angle \\
\hline \hline Laplacian, explicit & 12.96 & 100.21 \\
\hline Torsion Springs & 12.19 & 97.50 \\
\hline Laplacian, opt. based & 13.81 & 100.86 \\
\hline Conformal Energy & 12.93 & 99.79 \\
\hline Distortion Metric & 21.08 & 97.20 \\
\hline Squared Cotangents & 21.33 & 96.32 \\
\hline
\end{tabular}

The domain of the first test-case has also been chosen as an example to illustrate another effect of mesh smoothing. As shown in Figure 3.10 on page 85, the smoothing-functionals differ in the way they redistribute the nodes globally. $F_{\Delta}$ (and so $F_{c o n}$ and $F_{\text {tors }}$ ) produce high-quality triangles in the top-region, at the cost of the triangles in the quenched bottom region of the example domain, while $F_{\text {dist }}$ and $F_{\cot ^{2}}$ achieve a more fair distribution of acute angles.

Mesh-smoothing for parametric test-cases We will now apply the smoothingalgorithm to a parametric example. The torsion-spring energy will be used no longer.

Figure 3.11 on page 85 shows the development of triangulations of the surface shown in figure Figure 3.12(a) on page 86. The surface originates from a globally refined icosahedron inscribed into the unit-sphere. The resulting mesh was heavily deformed and afterwards refined, either globally (Figure 3.11(a) on page 85) or locally (Figure 3.11(b) on page 85).

The initial triangulation has some quite obtuse angles, resulting from the deformation of the underlying sphere. 
$\overline{\text { Figure 3.9 Distribution of angles with different smoothing-functionals. The colours }}$ indicate the element quality in terms of the size of the interior angles (blue is better).

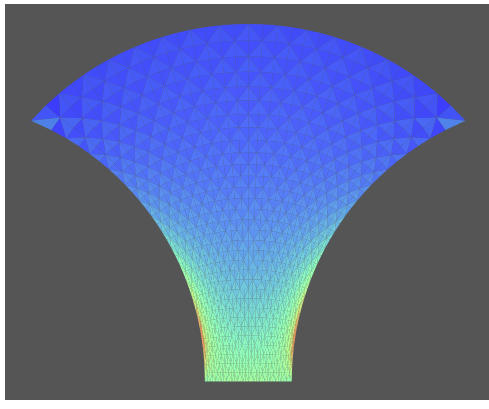

(a) Laplacian, explicit

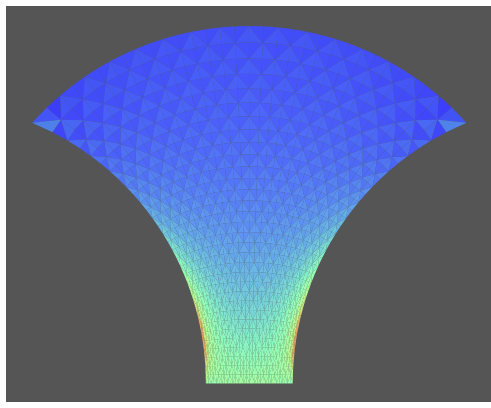

(d) Conformal Energy

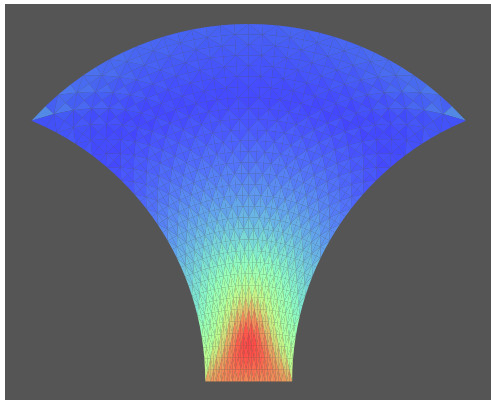

(b) Torsion springs

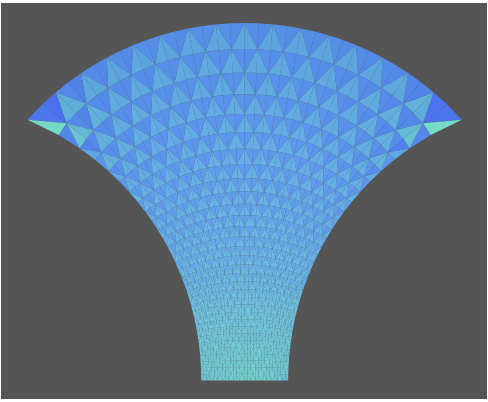

(e) Distortion Metric

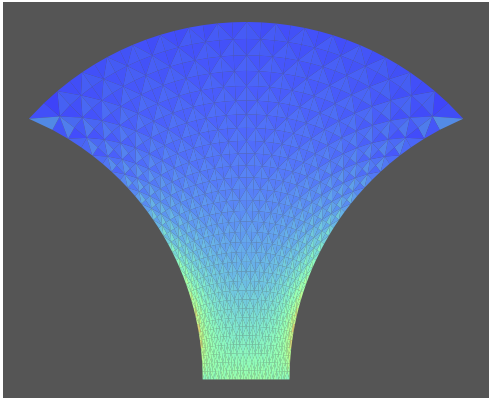

(c) Laplacian, opt. based

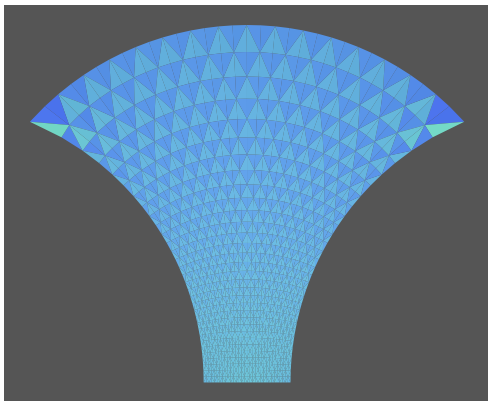

(f) squared cotangens

Figure 3.11 on the next page shows a big difference in the behaviour of the various smoothing-functionals with respect to the topology of the surface mesh. In the globally refined case the results of the more complicated smoothing-functionals $F_{\text {dist }}$ and $F_{\cot ^{2}}$ are much better than the results achieved by smoothing with the conformal energy $F_{\text {con }}$. Again, the diagrams demonstrate that there is only little difference between Laplacian smoothing and smoothing with the conformal energy.

Excessive mesh-smoothing - in this examples we were using 200 iterations - can severely change the node-distribution over the surface-mesh; the tips of the lobes in Figure 3.11 are only covered by a very coarse mesh after the smoothing-algorithm has finished. However, one notes that the smoothing-functionals tend to move nodes to regions of high curvature, where a high resolution is needed. A local mesh-adaption strategy can afterwards compensate the coarse-mesh structure in regions of low curvature.

As a final example, we present how the smoothing-functionals behave when applied to our path-tracking problem. This will also explain why we were using $F_{\cot ^{2}}$, defined in (3.16), rather than Laplacian smoothing (or the essentially equivalent conformal energy $\left.F_{c o n}\right)$. For the test we chose the two-lobed family of solutions which branches off from the exact solutions found by Beer (see Section 1.4 on page 16). This is the simplest noncylinder-symmetric branch but it already develops a distinct constriction around the origin when the angular velocity $\omega$ approaches 0 , as shown in Figure 3.14 on page 87. 
Figure 3.10 Top and bottom parts of Figure 3.9 on the preceding page, bottom part magnified. Mesh-smoothing changes the local node-density

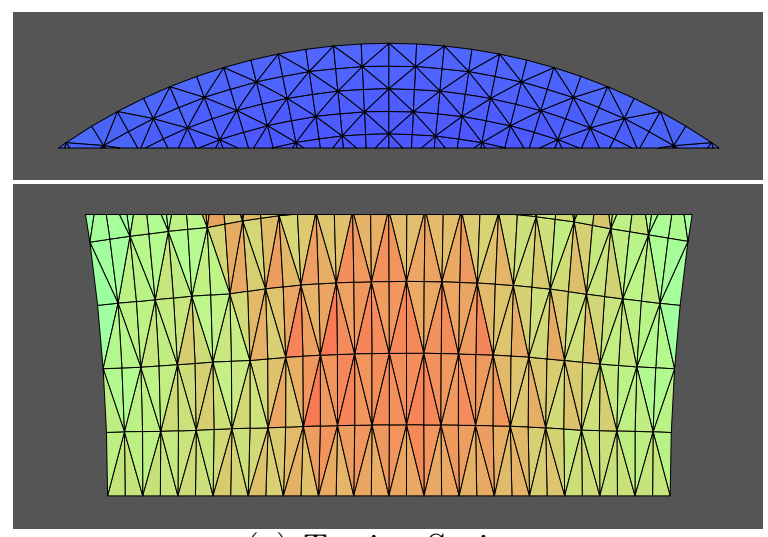

(a) Torsion Springs
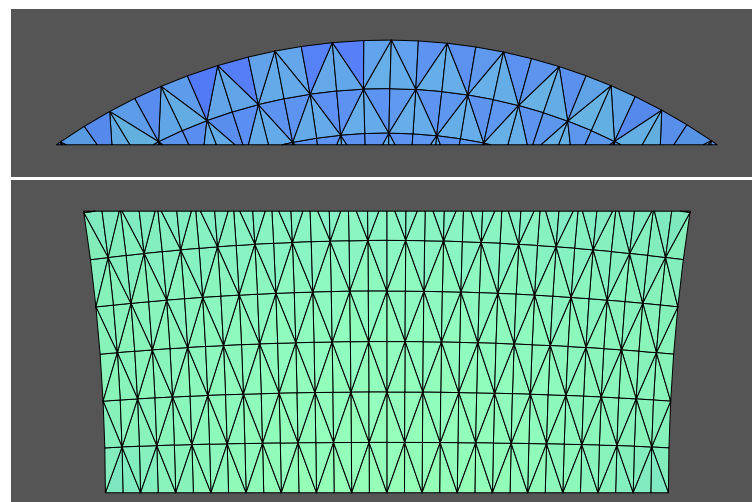

(c) Distortion Metric

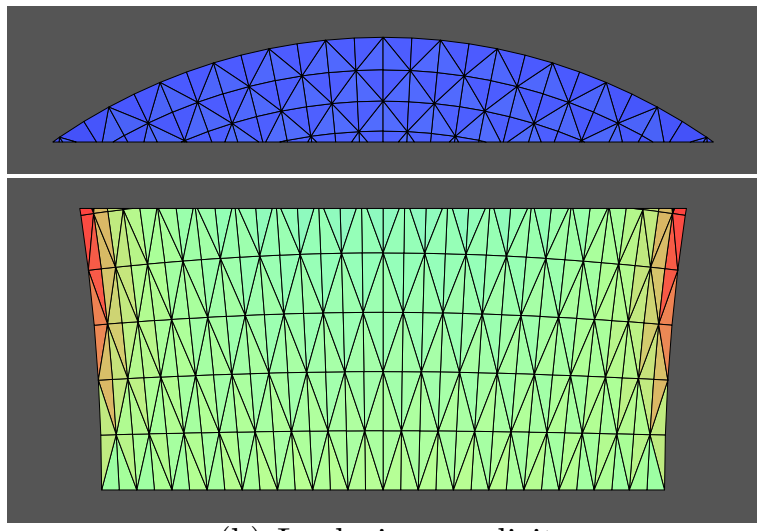

(b) Laplacian, explicit

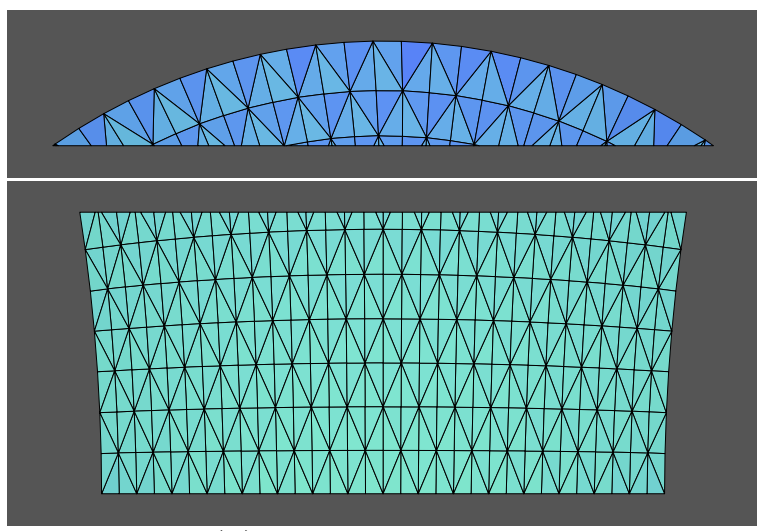

(d) Squared Cotangens

Figure 3.11 Mesh-smoothing for the parametric example mesh shown in Figure 3.12 on the next page. The two diagrams show the development of the maximal angle for the indicated smoothing-functionals for the surface meshes shown in Figure 3.12(a) on the following page.

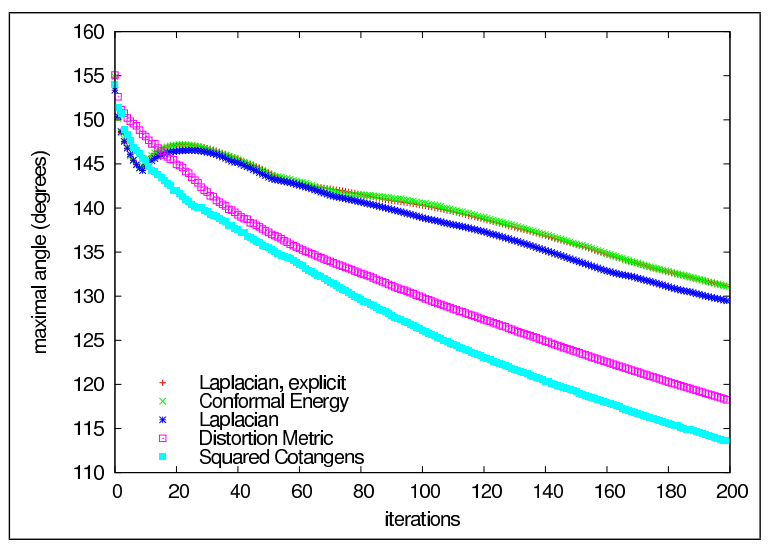

(a) Globally refined mesh

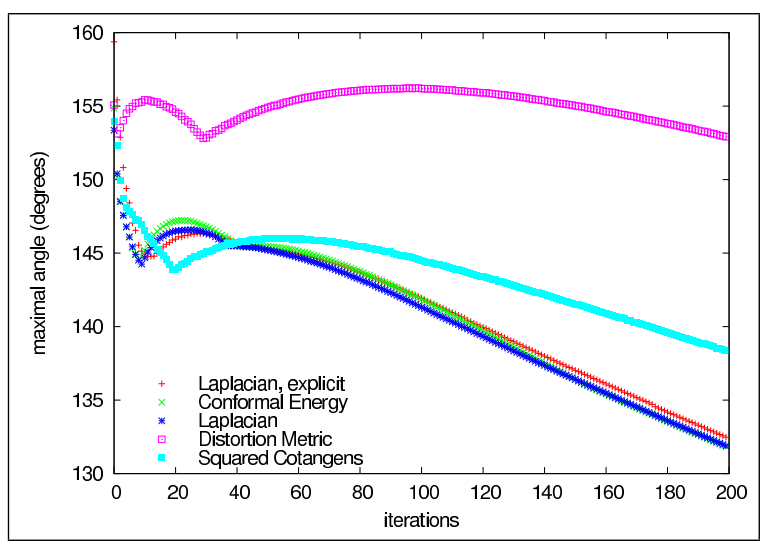

(b) Locally refined mesh 
Figure 3.12 Geometry and smoothed-meshes for a parametric example. Only the locally refined case is shown. The colours reflect the local mesh-quality (dark blue is better).

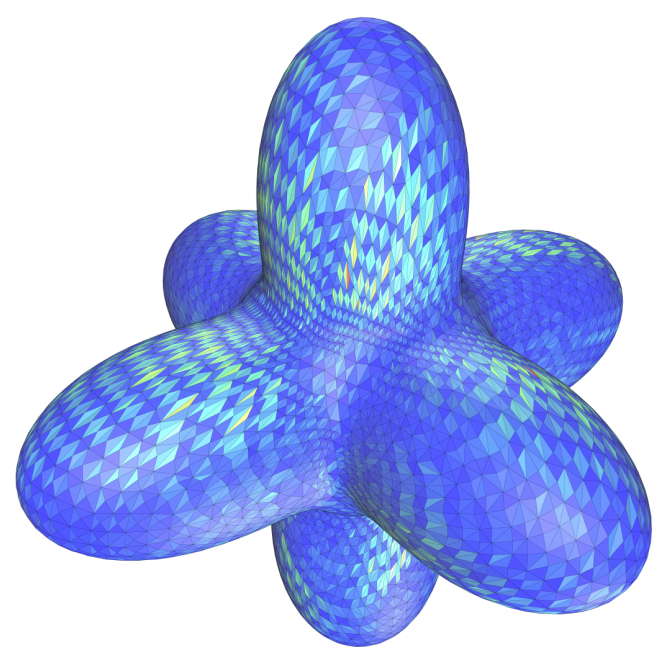

(a) Initial mesh

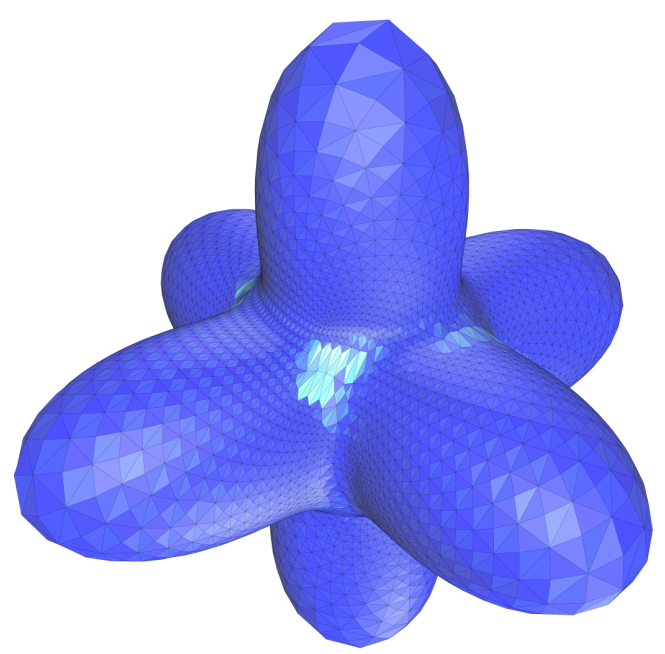

(c) Distortion Metric

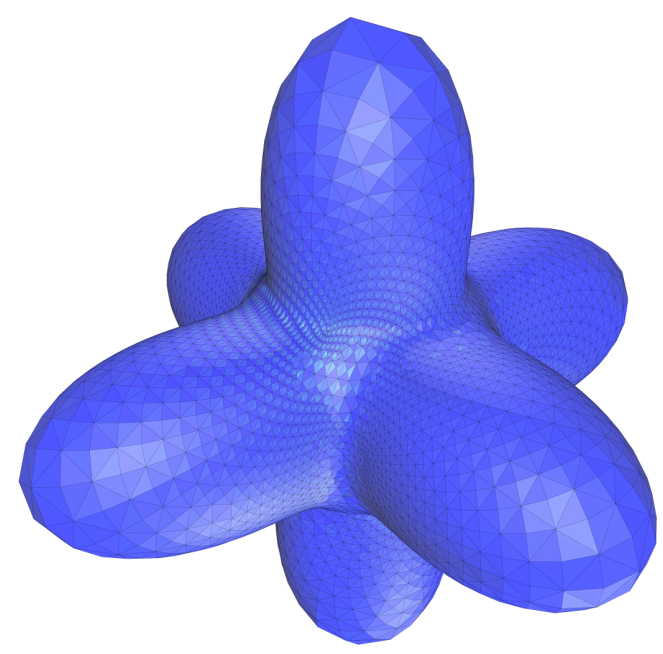

(b) Conformal Energy

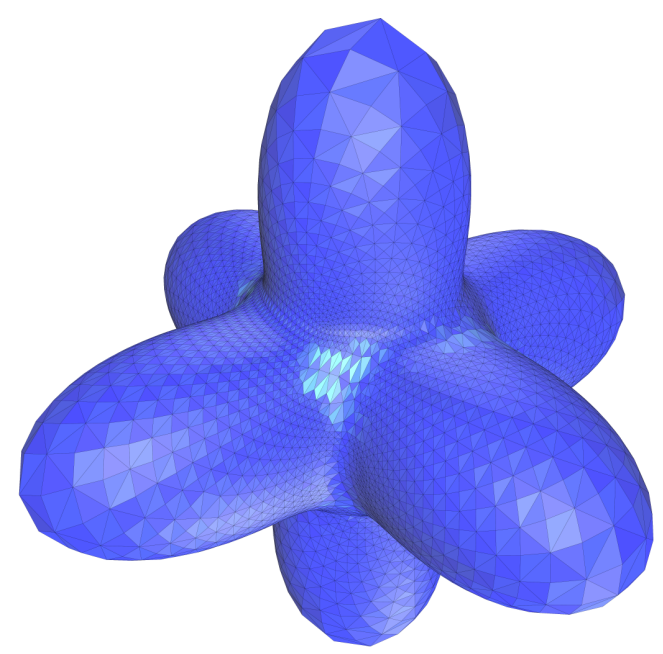

(d) Squared Cotangens 
$\overline{\text { Figure 3.13 Different smoothing-functionals acting on the two-lobed family. The goal }}$ was to keep the minimal angle above $30^{\circ}$, starting from very regular triangulation at $\omega \approx 0.55$. (a) shows that smoothing with $F_{d i s t}$ and $F_{\cot ^{2}}$ achieve this goal very well.

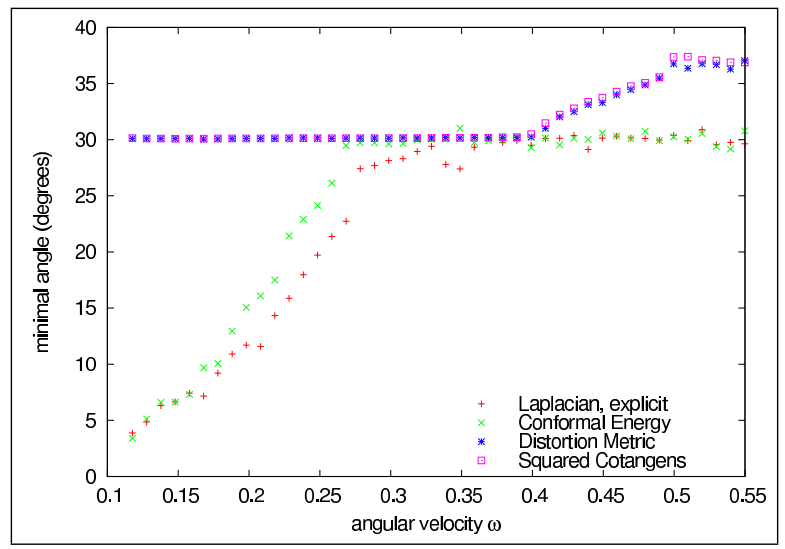

(a) Development of the minimal angle

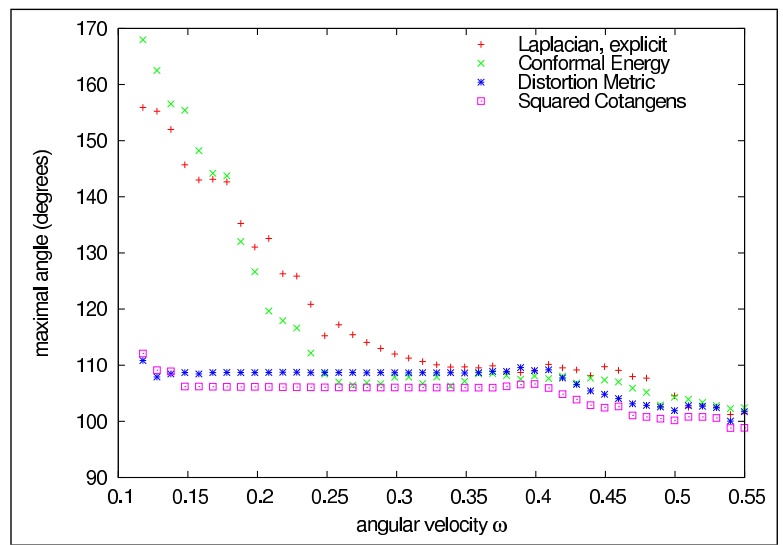

(b) Development of the maximal angle

$\overline{\text { Figure 3.14 Result of path-tracking using optimisation based mesh-smoothing with }}$ either $F_{c o n}$ or $F_{\cot ^{2}}$

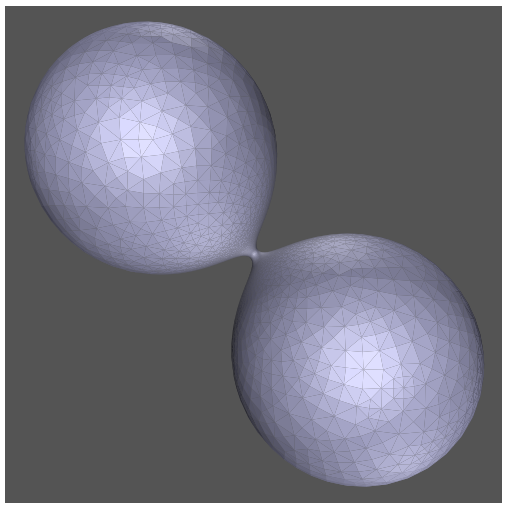

(a) Drop shape at $\omega \approx 0.12$

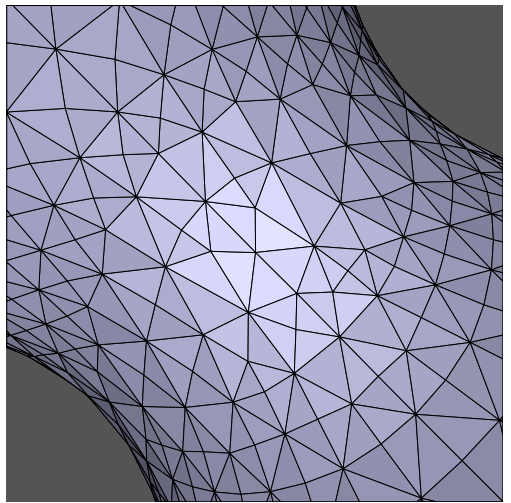

(b) Magnified central region of (a), smoothed using $F_{\cot ^{2}}$

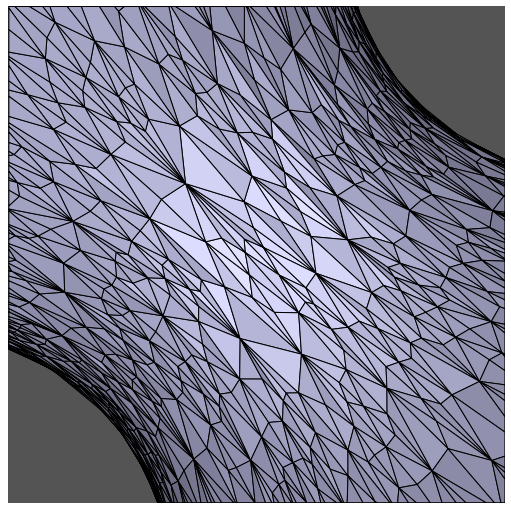

(c) Magnified central region of (a), smoothed using $F_{\text {con }}$

The test-runs started at $\omega \approx 0.55$ and stopped at $\omega \approx 0.12$. Figure 3.13 shows that neither optimisation based smoothing with the Conformal Energy $F_{\text {con }}$ nor explicit Laplacian smoothing are able to keep the mesh in good shape, and the corresponding test-runs broke down at $\omega \approx 0.12$, the last value shown in Figure 3.13.

While the same diagram also shows that there is little difference between $F_{\text {dist }}$ and $F_{\cot ^{2}}$, we nevertheless chose $F_{\cot ^{2}}$ as it yielded better results for annular drop shapes. We will not discuss this here further, but the example for the planar Figure 3.10 on page 85 and the first parametric test-case Figure 3.11 on page 85 already indicate that $F_{\cot ^{2}}$ yields slightly better results than $F_{d i s t}$, though at a higher computational cost. 


\subsubsection{Projection to discretised surfaces}

As a part of the mesh-smoothing Algorithm 3.4.4 on page 76 we need to project the displaced nodes back to $\Gamma_{h, 0}$. So we need a projection

$$
\Pi_{\Gamma_{h, 0}}: U\left(\Gamma_{h, 0}\right) \rightarrow \Gamma_{h, 0}
$$

which maps points from a neighbourhood $U\left(\Gamma_{h, 0}\right)$ of $\Gamma_{h, 0}$ onto the surface.

A crucial part of our mesh-smoothing algorithm is the method described in [SCHMid 1993]. We give a detailed description as it has to be analysed carefully to be able to implement it in a stable way.

In order to implement the projection $\Pi_{\Gamma_{h, 0}}$ efficiently, one first searches for the triangle onto which a point $P$ will be projected. To this aim for points $P \in \Gamma_{h, k}$ one maintains mappings $P \mapsto T_{P}^{k}$, where $T_{P}^{k} \subset \Gamma_{h, 0}$ is the element that $P$ was projected to in the previous iteration of the smoothing algorithm - that is, when $\Gamma_{h, k}$ was generated $(k \in \mathbb{N})$. For $T_{P}^{0}$ one just selects one of the elements adjacent to $P$.

$T_{P}^{k}$ will be close to the element to which $P \in \Gamma_{h, k}$ will be projected in the $k$-th run of the smoothing-algorithm, because the amount of displacement caused by the single-step method in each iteration step is not very large.

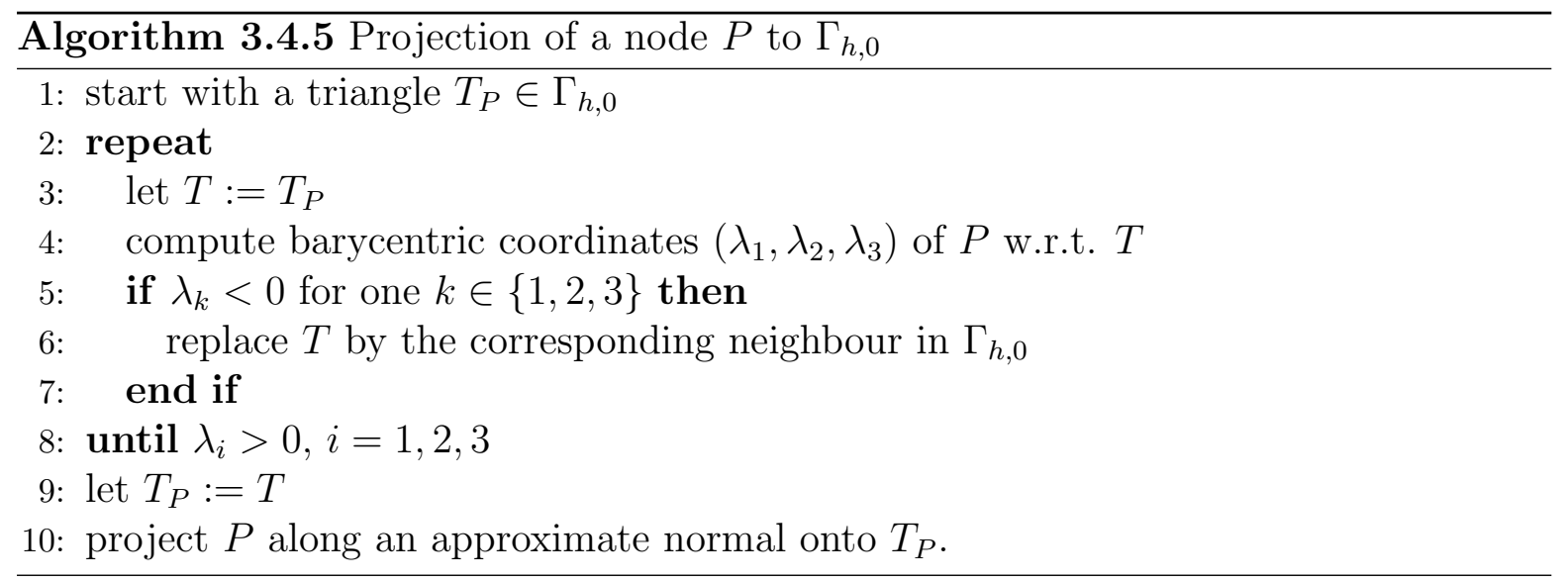

Starting with $T_{P}^{k}$, one uses Algorithm 3.4.5 to find $T_{P}^{k+1}$ and to compute $\Pi_{\Gamma_{h, 0}}(P)$. Line 4 of Algorithm 3.4.5 - the computation of the barycentric coordinates - is the crucial point and will be more detailed now.

Linear case First, we will present the computation of the barycentric coordinates for the linear case, where $\Gamma_{h, 0}$ is parametrised locally by linear Lagrange finite elements; the method will later be extended to parametrisations of higher polynomial degree.

Let $P$ be a point off the surface and $T \subset \Gamma_{h, 0}$ an element of the triangulation of $\Gamma_{h, 0}$ with vertices $P_{i}, i=1,2,3$. Let $\nu_{i}, i=1,2,3$ be the approximate normals (refapproxnormals) at the vertices of $T$.

The idea is to compute the barycentric coordinates $\lambda=\left(\lambda_{0}, \lambda_{1}, \lambda_{2}\right)$ of $P$ with respect to the triangle $\hat{T}$ parallel to $T$, which is inscribed into the skew-prism defined by the approximate normals $\nu_{i}, 1 \leq i \leq 3$, of $T$ (see Figure 3.15). 
Let $\nu_{T}$ (not shown in the figure) be the (non-approximate) unit-normal of $T$ and

$$
Q_{i}:=P_{i}+\frac{\left\langle P-P_{3}, \nu_{T}\right\rangle}{\left\langle\nu_{i}, \nu_{T}\right\rangle} \nu_{i}
$$

be the vertices of $\hat{T}$.

Of course, instead of $P_{3}$ any of the other two vertices of $T$ could also be used as origin.

$\lambda$ can then be computed by solving the linear system

$$
\left\{\begin{array}{l}
\lambda_{1}\left(Q_{1}-Q_{3}\right)+\lambda_{2}\left(Q_{2}-Q_{3}\right)=\left(P-Q_{3}\right) \\
\lambda_{3}=1-\lambda_{1}-\lambda_{2}
\end{array}\right.
$$

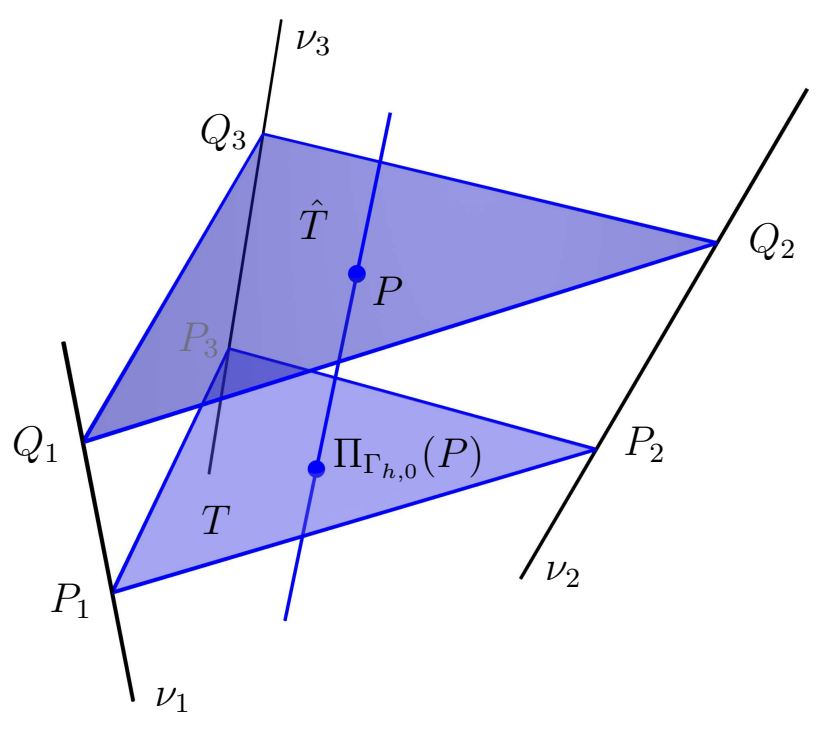

Figure 3.15 Projection to triangulations

(3.17) has a unique solution, provided that the skew-prism is not degenerated. If the barycentric coordinates are all positive, then $P$ is located inside $\hat{T}$.

In the linearly parametrised case, the projection $\Pi_{\Gamma_{h, 0}}^{\text {lin }}$ of $P$ to $\Gamma_{h, 0}$ is then simply defined as

$$
\Pi_{\Gamma_{h, 0}}^{l i n}:=\sum_{i=1}^{3} \lambda_{i} P_{i}
$$

[SCHMiDT 1993] observed that in some cases the Projection-Algorithm 3.4.5 on the preceding page will run into loops. As we found out the reason for this are not mere rounding errors. The skew-prisms defined by the approximate normals at the vertices of each element do not constitute a unique subdivision of a neighbourhood of $\Gamma_{h, 0}$, as was intended.

This is illustrated in Figure 3.16 on the following page. The walls of the skew-prisms are pieces of ruled surfaces, in general being parts of hyperboloids. The skew-prisms intersect each other and leave holes between them.

The errors are still small as long as the mesh is fine enough and the jump of the element normals between neighbouring elements are not too large. However, the errors are several orders of magnitude larger than rounding errors, and they can become large in regions where the mesh is not fine enough to resolve the curvature of the surface (in the sense that the jump of the element normals between neighbouring triangles is large).

The algorithm has to take this into account; when it runs into a loop, it just picks one of the elements of the loop-path as target element and projects to an edge which corresponds to a negative barycentric coordinate $\lambda_{k^{*}}$ and "repairs" the remaining two barycentric coordinates by adding $-\frac{1}{2} \lambda_{k^{*}}$. 
$\overline{\text { Figure 3.16 Projection to discrete surfaces. In general the skew-prisms overlap and }}$ leave holes between them, they are ruled surfaces.

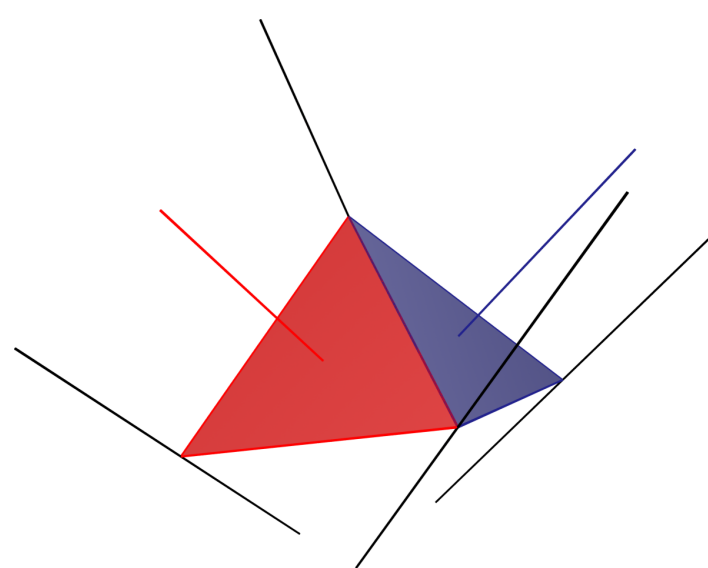

(a) Elements, face normals (coloured), approximate normals (black)

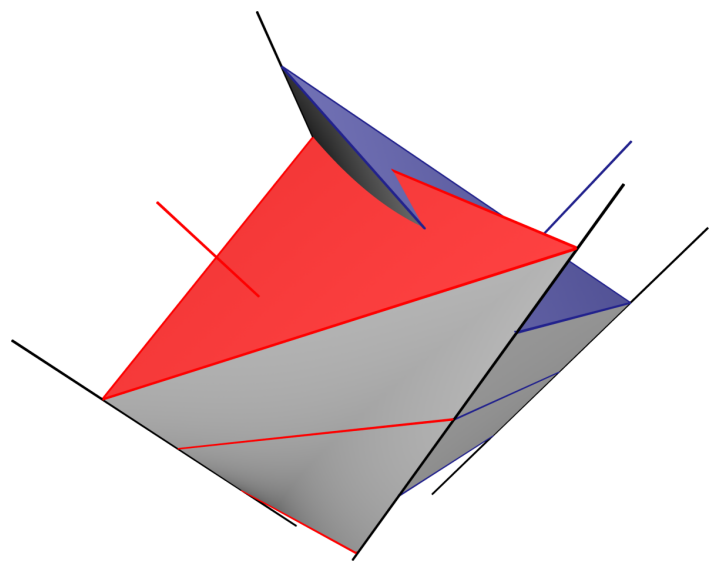

(c) Overlapping prisms, top view

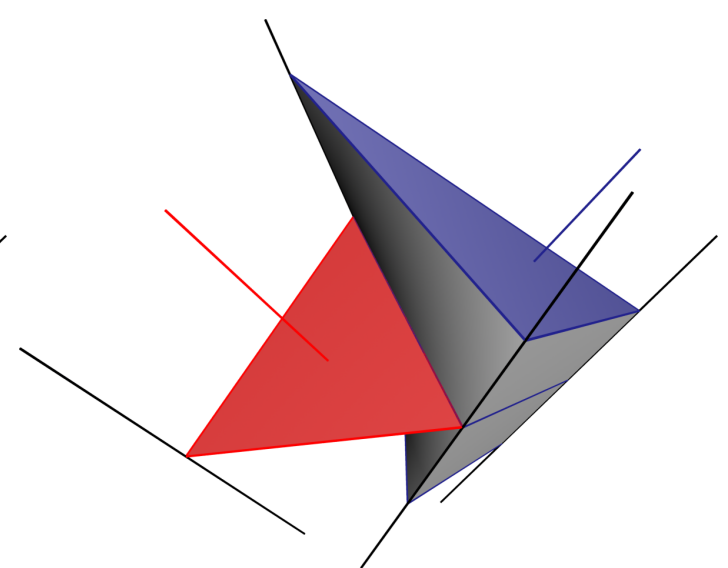

(b) Single skew prism spanned by approximate normals

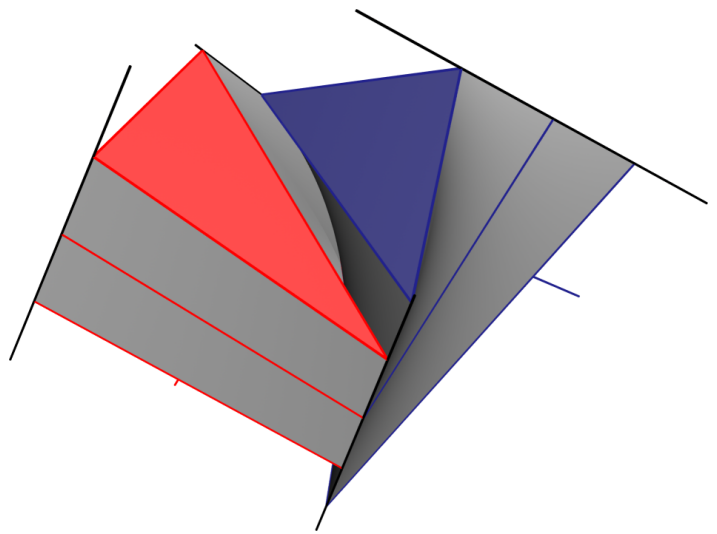

(d) Bottom view

It would have been possible to avoid the problems shown in figure Figure 3.16 by not defining the skew-prism by parallel translation of the elements $T$ of $\Gamma_{h, 0}$. Instead, one could have defined the vertices $Q_{i}$ of the top triangle $\hat{T}$ in Figure 3.15 on the preceding page by moving the points $P_{i}$ the same distance along the average normals $\nu_{i}$ until $\hat{T}$ contained the point $P$. But this would lead to a quadratic equation for the barycentric coordinates, which would have been computationally more costly to solve than solving the linear equation (3.17).

Higher order parametrisations Still following [SCHMIDT 1993], we first proceed as in the linear case and determine $T_{P}$ and and the barycentric coordinates $\left(\lambda_{1}, \lambda_{2}, \lambda_{3}\right)$ of $P$ with respect to the triangulation defined by the vertex nodes of $\Gamma_{h, 0}$. The goal is then to project $P$ along the direction of $g$ defined as the weighted mean value

$$
g:=\sum_{i=1}^{3} \lambda_{i} \nu_{i}
$$

to the non-linearly parametrised surface. 
The point where $G:=\{P+s g \mid s \in \mathbb{R}\}$ meets the element $T$ (if it meets it at all) is a zero of the function $d_{P, g, T}\left(\lambda_{1}, \lambda_{2}\right)$ defined by

$$
d_{P, g, T}\left(\lambda_{1}, \lambda_{2}\right):=\Psi_{T}\left(\lambda_{1}, \lambda_{2}\right)-P-\frac{\left\langle\Psi_{T}\left(\lambda_{1}, \lambda_{2}\right)-P, g\right\rangle}{\|g\|^{2}} g .
$$

Here $\Psi_{T}\left(\lambda_{1}, \lambda_{2}\right)$ denotes the parametrisation of $T$ over the reference simplex, choosing $\lambda_{1}$ and $\lambda_{2}$ as coordinates as in (3.17). $d_{P, g, T}\left(\lambda_{1}, \lambda_{2}\right)$ is the perpendicular line from $G$ to $\Psi_{T}\left(\lambda_{1}, \lambda_{2}\right)$, so it is a mapping from the reference triangle to $G^{\perp}$.

We can try to solve the non-linear equation (3.19) by a Newton iteration. For the case that one of the components of the solution $\lambda=\left(1-\lambda_{1}-\lambda_{2}, \lambda_{1}, \lambda_{2}\right)$ is negative, [SCHMIDT 1993] suggests to proceed to the corresponding neighbour as described in Algorithm 3.4.5 on page 88 .

\section{Figure 3.17 Projection to parametric surfaces, polynomial degree is 2}

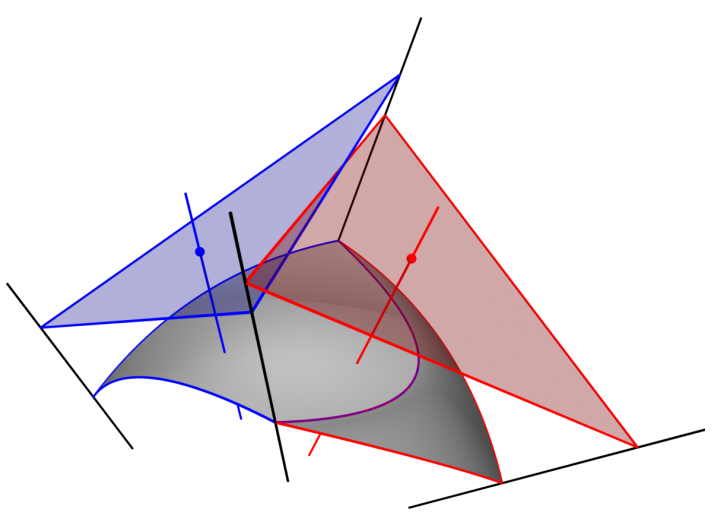

(a) Projection hits the "wrong" element

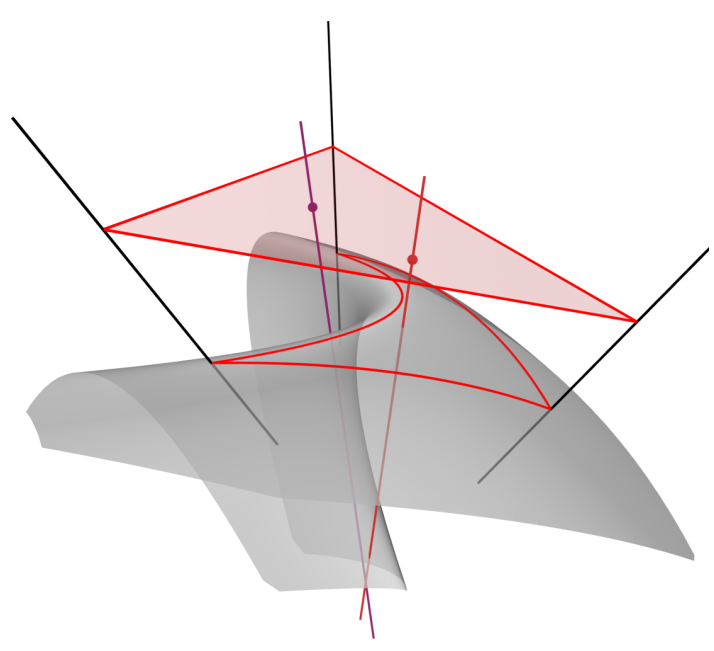

(b) Multiple or no "projections"

Unluckily, there are some complications. Two of the possible cases are illustrated in Figure 3.17, which shows quadratic parametrisations of two neighbouring elements. The straight lines in blue and red in the interior of the top-triangles indicate possible searchdirections $g$.

The blue line in Figure 3.17(a) shows the "good" case where the projection along $g$ hits the same element as found by the linear projection $\Pi_{\Gamma_{h, 0}}^{\text {lin }}$. The red line originated from the pale-red coloured top-triangle on the right, but the non-linear projection should end up in left the element with the blue boundary.

However, it is not that easy to for a computer to efficiently find out that the "blue" element is indeed the right one for the red line in Figure 3.17(a). This is demonstrated in Figure 3.17(b), which shows - slightly rotated to give a better view - the left element, where the quadratic parametrisation is extended beyond the boundaries of the element.

The left, dark blue line in Figure 3.17(b) does not hit the quadratic parametrised surface at all. In this case equation (3.19) does not provide information about a neighbour which might contain the projection. 
So it is not possible to apply the Projection-Algorithm 3.4.5 on page 88 blindly. To cope with this case, our implementation falls back to a brute-force search. If a solution of (3.19) cannot be found, traversing neighbouring elements until it succeeds.

This alone still does not suffice. The orange line on the right in Figure 3.17(b) on the preceding page hits the surface obtained by extending the quadratic parametrisation beyond the boundary of the element twice. This shows that a negative component of the barycentric coordinates obtained as a solution of (3.19) does not necessarily mean that the point should be projected to a neighbouring element.

So if the brute-force search still fails, the start-values for the Newton iteration are altered in the hope to eventually catch the correct solution.

This works in most cases. Otherwise, when a vertex-node cannot be projected, the node simply remains in place during the corresponding iteration of the smoothing-algorithm.

When non-vertex nodes cannot be projected, our implementation just skips the projection step. This means that the corresponding node remains in the place which is defined by the barycentric coordinates of its associated Lagrange node. In the case of 2-dimensional Lagrange elements this is just the mid-point of the edge which connects the neighbouring vertices. 


\section{Chapter 4}

\section{Numerical results}

In this chapter we present the results of our numerical experiments.

We will begin with experimental convergence tests with respect to the discretised Newton iteration described in Section 2.4. In particular, we present EOC-tests with respect to the real $\mathrm{L}_{2}$ and $\mathrm{H}^{1}$-errors for approximations of some explicitly know solutions. Furthermore, we will compare the ad-hoc error indicators introduced in Section 2.4.4 with the real $\mathrm{L}_{2}$ - and $\mathrm{H}^{1}$-errors. We use some members of the axially symmetric families of spheroidal and annular drop shapes discussed in Section 1.4.

In the last sections of this final chapter we will present the results of our experimental numerical computations. Our computations - although completely experimental - illustrate nevertheless the capabilities of our approach:

1. our algorithm does not rely on artificial symmetry constraints

2. it cannot suffer from coordinate singularities

3. it is able to treat drop shapes of arbitrary topology

The third point is a consequence of using a local parametrisation over the reference element. We stress that 3. does not mean that we are able to compute "through changes of topology". Our numerical method preserves the topological class of the (discrete) surface because it preserves by construction the number of "holes" of the surface.

\subsection{Experimental convergence tests}

\subsubsection{Convergence of the discrete Newton iteration}

For a sequence of iso-parametric triangulations $\mathcal{T}_{h_{j}}$ we define the experimental order of convergence $(E O C)$ - as usual - by

$$
E O C_{j}:=\frac{\log \frac{\mathcal{E}_{j}}{\mathcal{E}_{j+1}}}{\log \frac{h_{j}}{h_{j+1}}},
$$


where $\mathcal{E}_{j}:=\left\|u-u_{h_{j}}\right\|$ denotes the error between the discrete solution $u_{h_{j}}$ and the exact solution $u$ in any appropriate norm.

Table 4.1 Errors and $E O C$ s for a spheroidal drop shape at $\omega \approx 0.7138$

\begin{tabular}{|l||l|l||l|l||l|l||l|l|}
\hline$h_{\max }$ & $\left\|\Gamma-\Gamma_{h}\right\|_{\mathrm{L}_{2}}$ & $E O C$ & $\left\|\Gamma-\Gamma_{h}\right\|_{\mathrm{H}^{1}}$ & $E O C$ & $\left\|\Gamma-\Gamma_{h}\right\|_{\mathrm{L}_{\infty}}$ & $E O C$ & $\left\|\Gamma-\Gamma_{h}\right\|_{\mathrm{H}_{\infty}^{1}}$ & $E O C$ \\
\hline \hline 0.159 & 0.000323 &. & 0.013 &. & 0.000342 &. & 0.0115 &. \\
\hline 0.08 & $2.78 e-05$ & 3.58 & 0.00309 & 2.1 & $4.39 e-05$ & 3. & 0.00366 & 1.67 \\
\hline 0.0401 & $3.37 e-06$ & 3.04 & 0.000798 & 1.96 & $6.47 e-06$ & 2.76 & 0.00137 & 1.42 \\
\hline 0.02 & $4.26 e-07$ & 2.99 & 0.000202 & 1.98 & $1.05 e-06$ & 2.63 & 0.000457 & 1.59 \\
\hline 0.01 & $5.38 e-08$ & 2.99 & $5.07 e-05$ & 1.99 & $1.59 e-07$ & 2.71 & 0.00013 & 1.82 \\
\hline
\end{tabular}

Table 4.2 Errors and EOCs for an annular drop shape at $\omega \approx 0.7069$

\begin{tabular}{|l||l|l||l|l||l|l||l|c|}
\hline$h_{\max }$ & $\left\|\Gamma-\Gamma_{h}\right\|_{\mathrm{L}_{2}}$ & $E O C$ & $\left\|\Gamma-\Gamma_{h}\right\|_{\mathrm{H}^{1}}$ & $E O C$ & $\left\|\Gamma-\Gamma_{h}\right\|_{\mathrm{L}_{\infty}}$ & $E O C$ & $\left\|\Gamma-\Gamma_{h}\right\|_{\mathrm{H}_{\infty}^{1}}$ & $E O C$ \\
\hline \hline 0.17 & 0.00035 &. & 0.0148 &. & 0.000455 &. & 0.0115 &. \\
\hline 0.0849 & 0.000128 & 1.46 & 0.00763 & 0.954 & 0.000204 & 1.16 & 0.0124 & -0.115 \\
\hline 0.0425 & $1.58 e-05$ & 3.02 & 0.00183 & 2.07 & $3.52 e-05$ & 2.54 & 0.00409 & 1.61 \\
\hline 0.0213 & $2.06 e-06$ & 2.94 & 0.000449 & 2.03 & $5.82 e-06$ & 2.6 & 0.00116 & 1.81 \\
\hline
\end{tabular}

Table 4.1 and Table 4.2 show the $\mathrm{L}_{2^{-}}, \mathrm{L}_{\infty^{-}}, \mathrm{H}^{1}$ - and $\mathrm{H}_{\infty}^{1}$-errors for the approximation of a spheroidal and an annular shape (see Figure 4.1 on the next page), both members of the exactly known axially symmetric families of solutions to equation (2.6). The discrete surfaces were generated by the discrete Newton iteration described in the previous chapter using iso-parametric Lagrange-elements of polynomial degree 2. In each iteration of the convergence test the surface mesh was globally refined by doubly bisecting each element (see Section 3.3).

The EOC of the $\mathrm{L}_{2^{-}}$and $\mathrm{H}^{1}$-errors shown in the tables 4.1 and 4.2 is nearly optimal in the sense that it is close to the approximation order of the Lagrange interpolation operator, see Theorem 2.4.5 on page 47 .

We note that the first $E O C$-value is not always of the magnitude of the interpolation order which is explained by the fact that the initial triangulation used in the convergence tests was very coarse; in general EOC-tests are only meaningful for sufficiently refined meshes.

This is also illustrated by the diagrams shown in the Figures 4.2 to 4.5 on pages 96-99. The figures show the results of a test-run which started with a very coarse approximation of a sphere as initial surface for the path-tracking algorithm. Then the discrete path-tracking algorithm described in the previous chapter (see the flow-chart shown in Algorithm 2.6.1 on page 58) generated an initial family of coarse iso-parametric surfaces approximating the exact axially symmetric solutions described in Section 1.4.

Each of the coarse initial iso-parametric triangulations was then globally refined effectively halving the mesh-constant in each refinement step. The labels "level $i$ " to the right of the keys at the bottom of the figures denote the $i^{\text {th }}$ refinement-step, so the maximal 


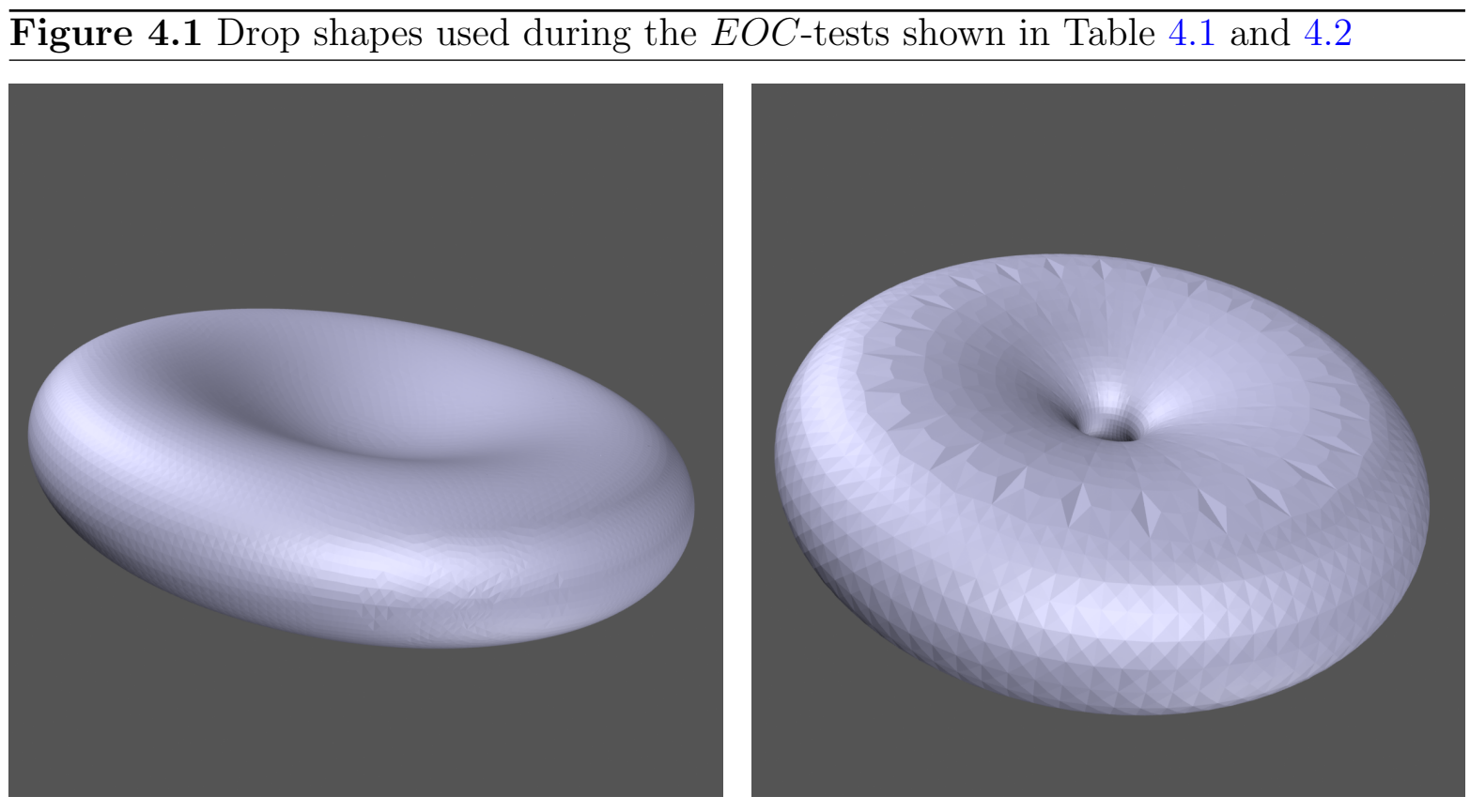

mesh-width of a triangulation at "level 5" is approximately 16-times smaller than the mesh-width of the corresponding "level 1" triangulation.

The final value of $\omega$ in the figures on the following pages is close to the value which marks the turning point where the spheroidal family bends back to meet the annular drop shapes (see Figure 1.5 on page 17).

Figure 4.2 shows the $E O C$ for the $\mathrm{L}_{2}$ - and $\mathrm{H}^{1}$-errors while tracking the axially-symmetric family of spheroidal drop-shapes. For the finer meshes the $E O C$ seems again to approach the approximation order of the Lagrange interpolation operator, in accordance with the behaviour shown in Table 4.1 and Table 4.2.

\subsubsection{Accuracy of the error indicators}

In Section 2.4.4 we have introduced two residual ad-hoc error indicators for controlling the local adaption of the surface grids. We repeat them here for the sake of clarity:

$$
\begin{aligned}
& \eta_{T, 2}^{2}:=C_{0}^{2} h_{T}^{4}\left\|-\Delta_{\Gamma_{h}} \operatorname{id}_{\Gamma_{h}}-\left(4 \omega^{2} r^{2}\right) \nu_{\Gamma_{h}}\right\|_{\mathrm{L}_{2}(T)}^{2}+C_{2}^{2} h_{T}^{3}\left\|\left[\left[\nu_{\Gamma_{h}}\right]\right]\right\|_{\mathrm{L}_{2}(\partial T)}^{2} \\
& \eta_{T, 1}^{2}:=\tilde{C}_{0}^{2} h_{T}^{2}\left\|-\Delta_{\Gamma_{h}} \operatorname{id}_{\Gamma_{h}}-\left(4 \omega^{2} r^{2}\right) \nu_{\Gamma_{h}}\right\|_{\mathrm{L}_{2}(T)}^{2}+\tilde{C}_{1}^{2} h_{T}\left\|\left[\left[\nu_{\Gamma_{h}}\right]\right]\right\|_{\mathrm{L}_{2}(\partial T)}^{2}
\end{aligned}
$$

By summing up the local contributions of each element of the iso-parametric triangulations $\mathcal{T}_{h}$ we arrive at the global quantities

$$
\eta_{1}:=\sqrt{\sum_{T \in \mathcal{T}_{h}} \eta_{T, 1}^{2}} \text { and } \eta_{2}:=\sqrt{\sum_{T \in \mathcal{T}_{h}} \eta_{T, 2}^{2}},
$$

which we use to measure the error of the discretisation in the path-tracking algorithm. More specifically, either the value $\eta_{1}$ or $\eta_{2}$ is used as the stopping criterion for the mesh-adaption in Algorithm 2.6.1 on page 58. 
Figure 4.2EOC for $\mathrm{L}_{2^{-}}$and $\mathrm{H}^{1}$-errors while tracking the spheroidal family

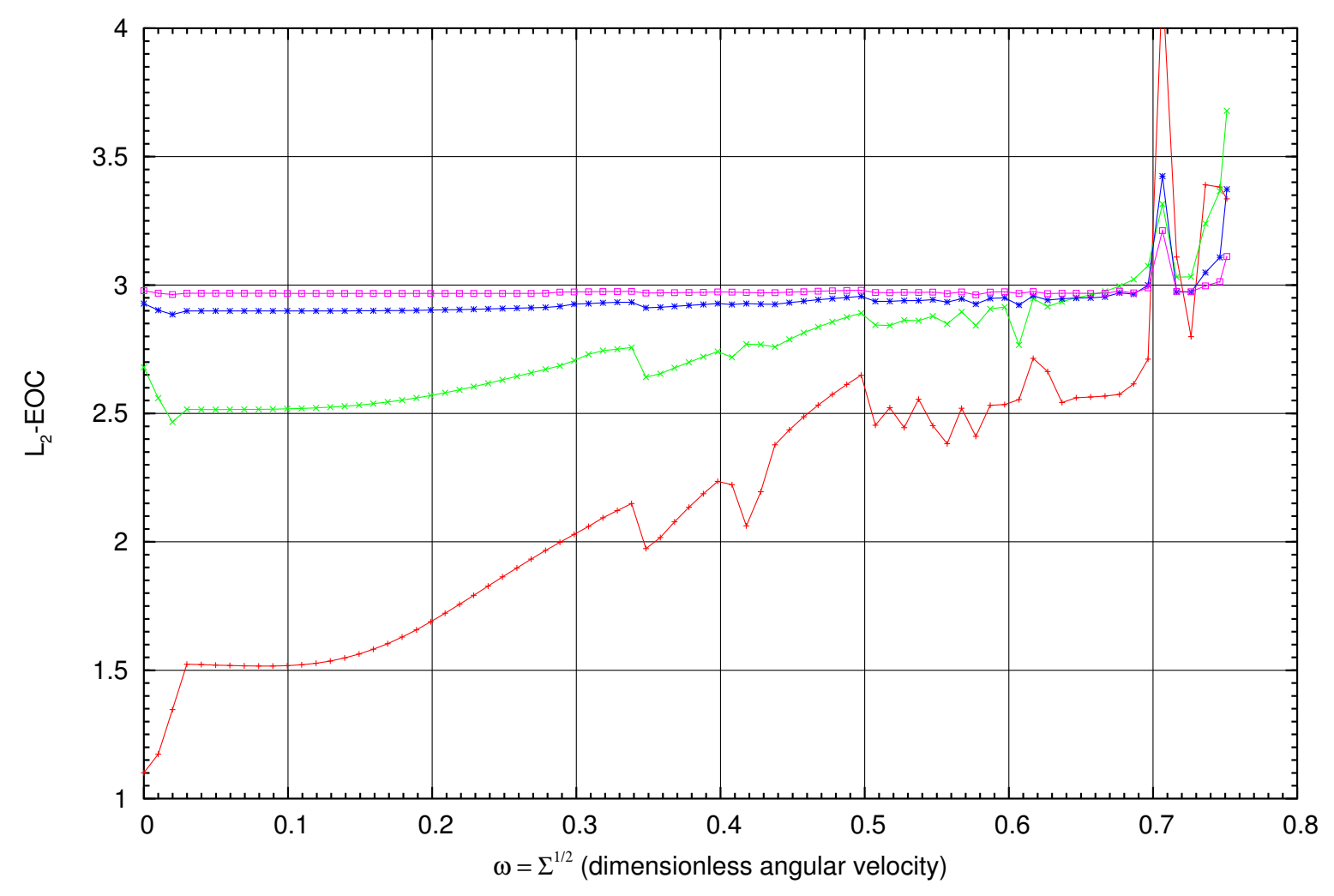
+ level 2
* level 3
* level 4
$\rightarrow$ level 5

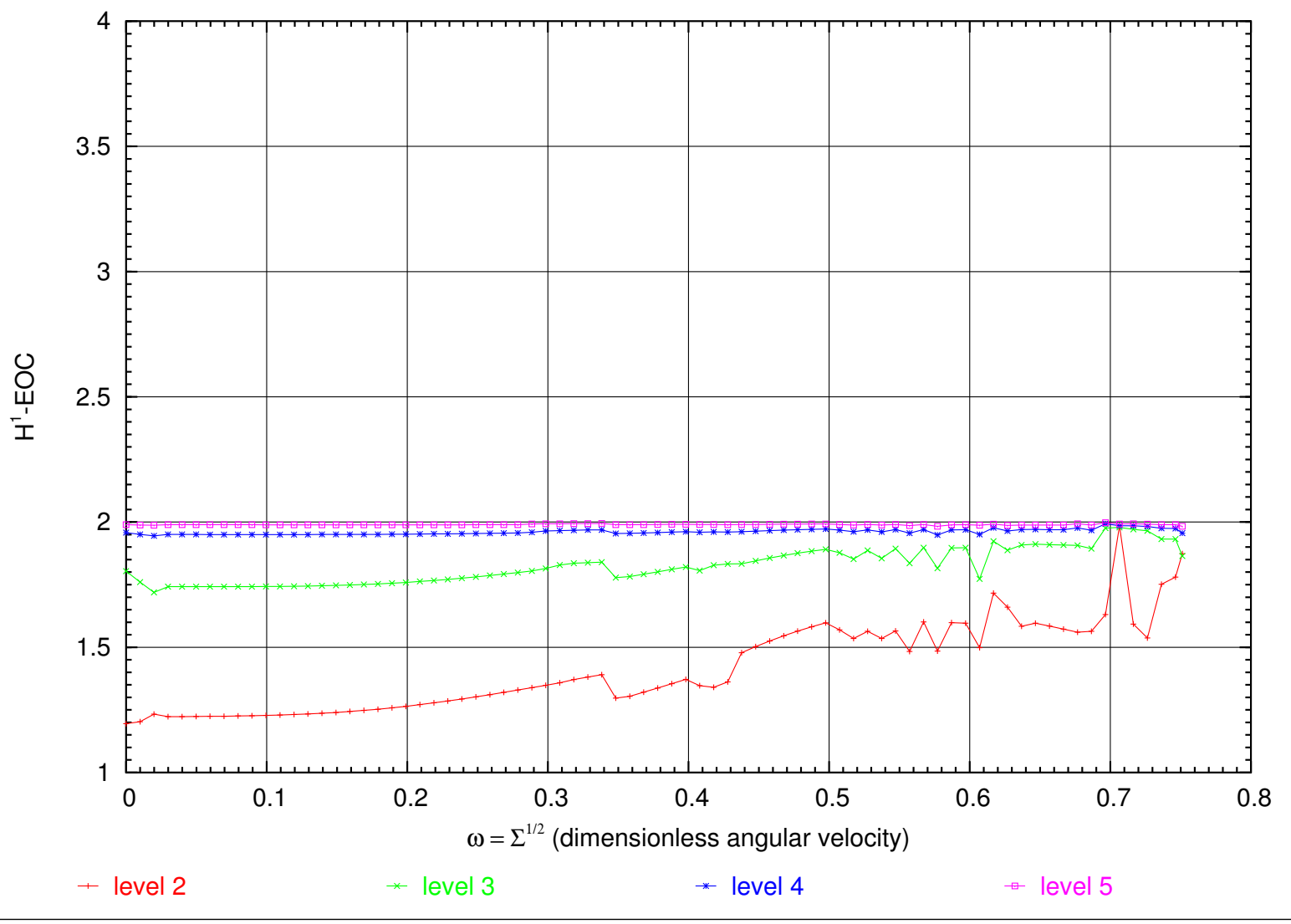


It is important to understand that it is not possible to determine constants $C_{0}$ and $C_{1}$ such that either $\eta_{1}$ or $\eta_{2}$ approximate the real error uniformly in $\omega$ because we compute the errors and the error indicators with respect to the $\mathrm{L}_{2^{-}}$and $\mathrm{H}^{1}$-spaces on each surface. Those spaces are isomorphic for smooth surfaces of the same topological type but they are not isometric. Moreover, the iso-parametric finite element spaces $W_{h}$ are not even isomorphic for the different discrete surfaces generated during the path-tracking if the mesh is refined or coarsened in between.

Finally, Figure 4.4 on the following page and Figure 4.5 on page 99 show the values of the real errors and the error indicator $\eta_{2}$ and $\eta_{1}$ using a logarithmic scale on the ordinate.

We note that the "noise" at $\omega \approx 0.71$ in some of the diagrams is caused by the bifurcation point which leads to the 3-lobed family.

\subsubsection{Eigenvalue computation}

Although the accuracy of eigenvalue computation is an important issue for the pathtracking algorithm - because it determines the position of the bifurcation points - we will be rather brief here. Figure 4.3 shows the first 5 eigenvalues of the linearised Euler-Lagrange equation for some of the drop-shapes computed during the convergence tests discussed in the previous two sections. We note that the figure actually shows the smallest 5 eigenvalues for 4 levels of global refinement - though this is hardly recognisable in the diagram on the left. The right diagram is just a magnified part of the left diagram and shows the $5^{\text {th }}$ eigenvalue. Still only the coarsest discretisation - the red line - can be clearly distinguished from all the others which are just plotted one over another.

$\overline{\text { Figure 4.3 The first five eigenvalues, computed for some of the shapes used for the }}$ experimental convergence tests shown on the pages before
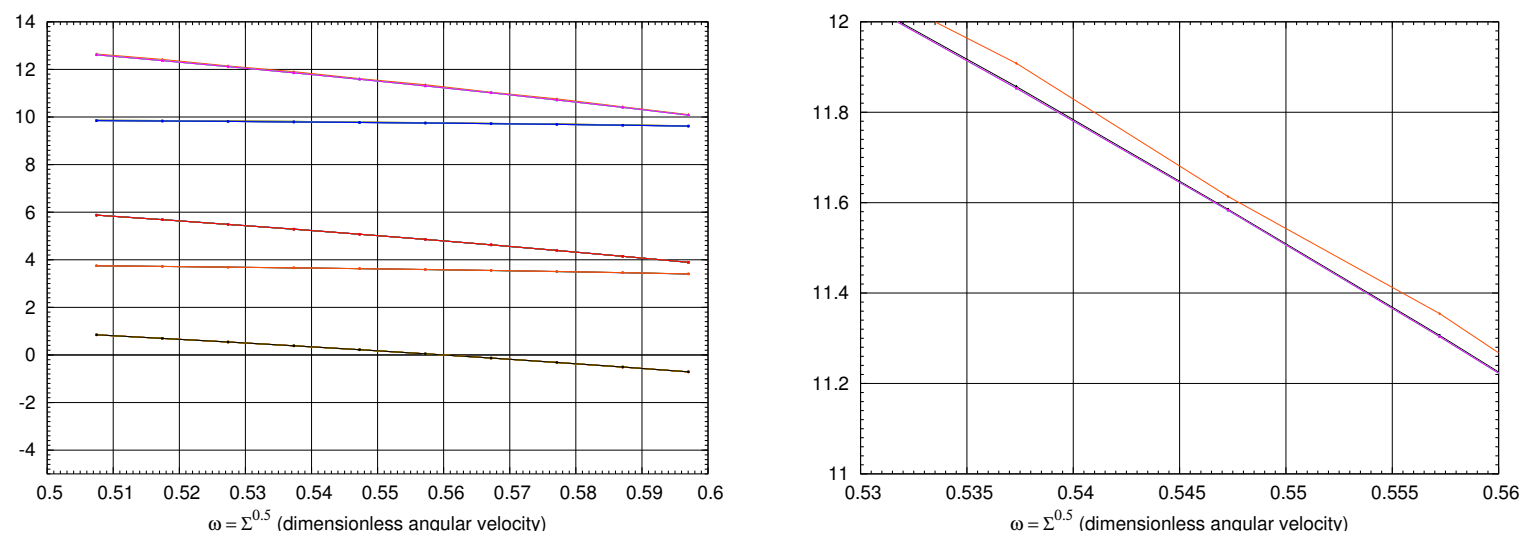
Figure $4.4 \mathrm{~L}_{2}$-errors and error-indicators while tracking the spheroidal family
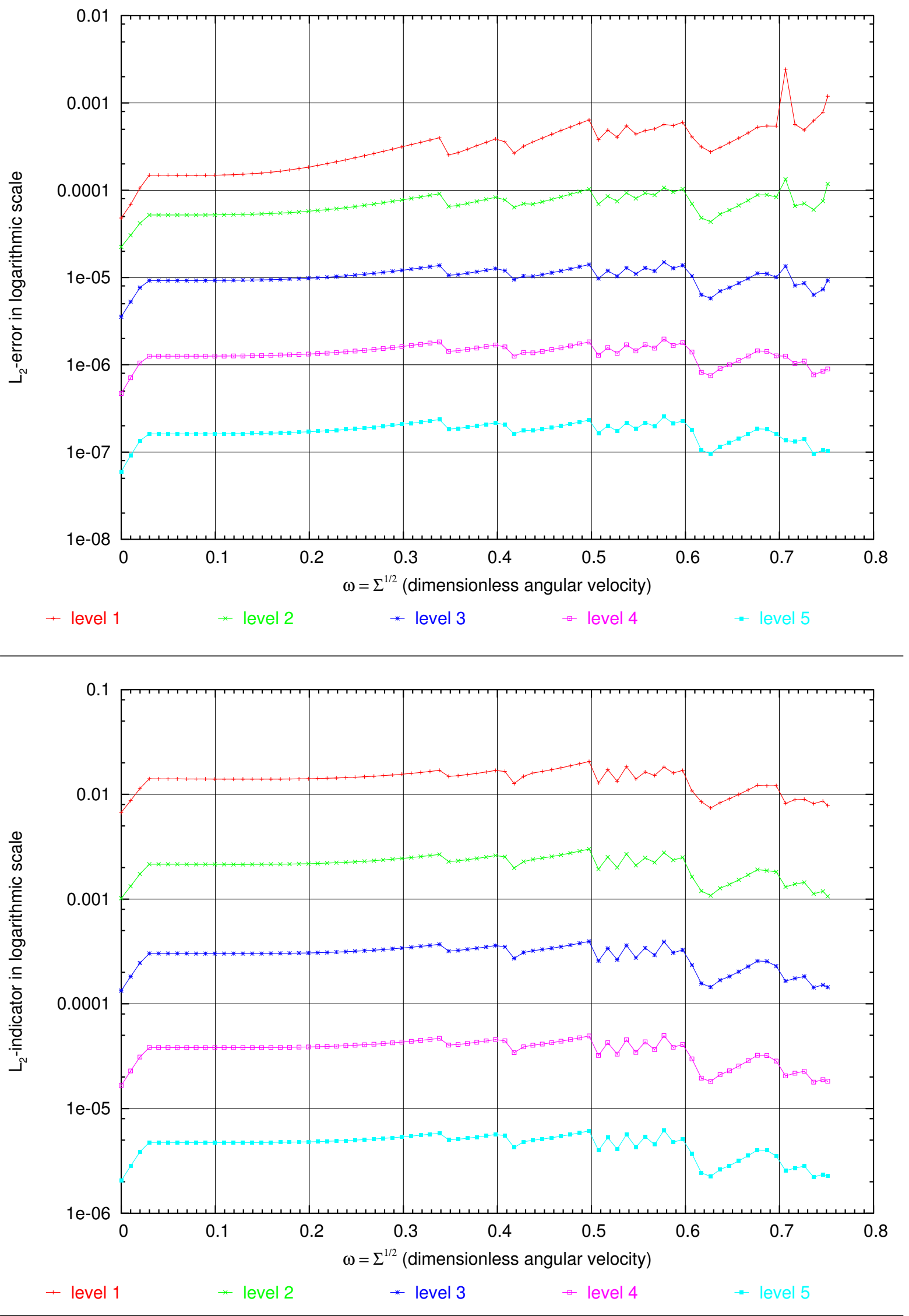

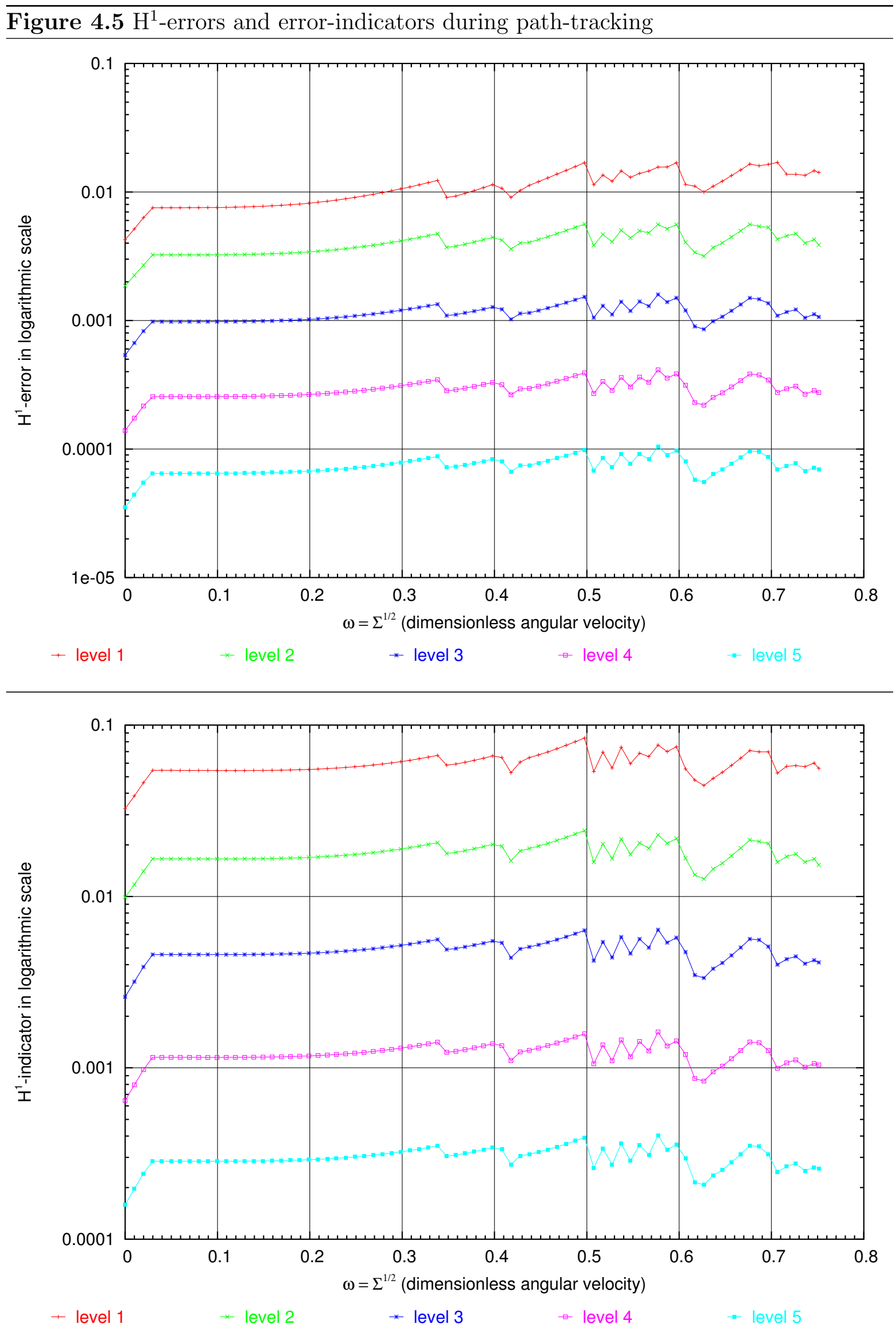


\subsection{Bifurcation diagrams and drop-shapes}

We start this section by comparing the bifurcation diagram Figure 4.6 - computed by [BROwn and SCRIVEN 1980a] - with the results of our own computations. Brown and Scriven were investigating numerically the bifurcations from the axially symmetric family of spheroidal shapes (see Section 1.4). We remark that Figure 4.7 on the next page showing the results of our own numerical experiments - has its axis interchanged with respect to Figure 4.6. Generally, we illustrate the bifurcations by plotting the angular momentum $L$ against the angular velocity $\omega$. Using either $(\omega, L)$ - or $(L, \omega)$-diagrams is a natural choice because $L$ is the proper parameter to describe freely rotating drops while the equations for the equilibrium shapes take as simpler when choosing $\omega$ as parameter.

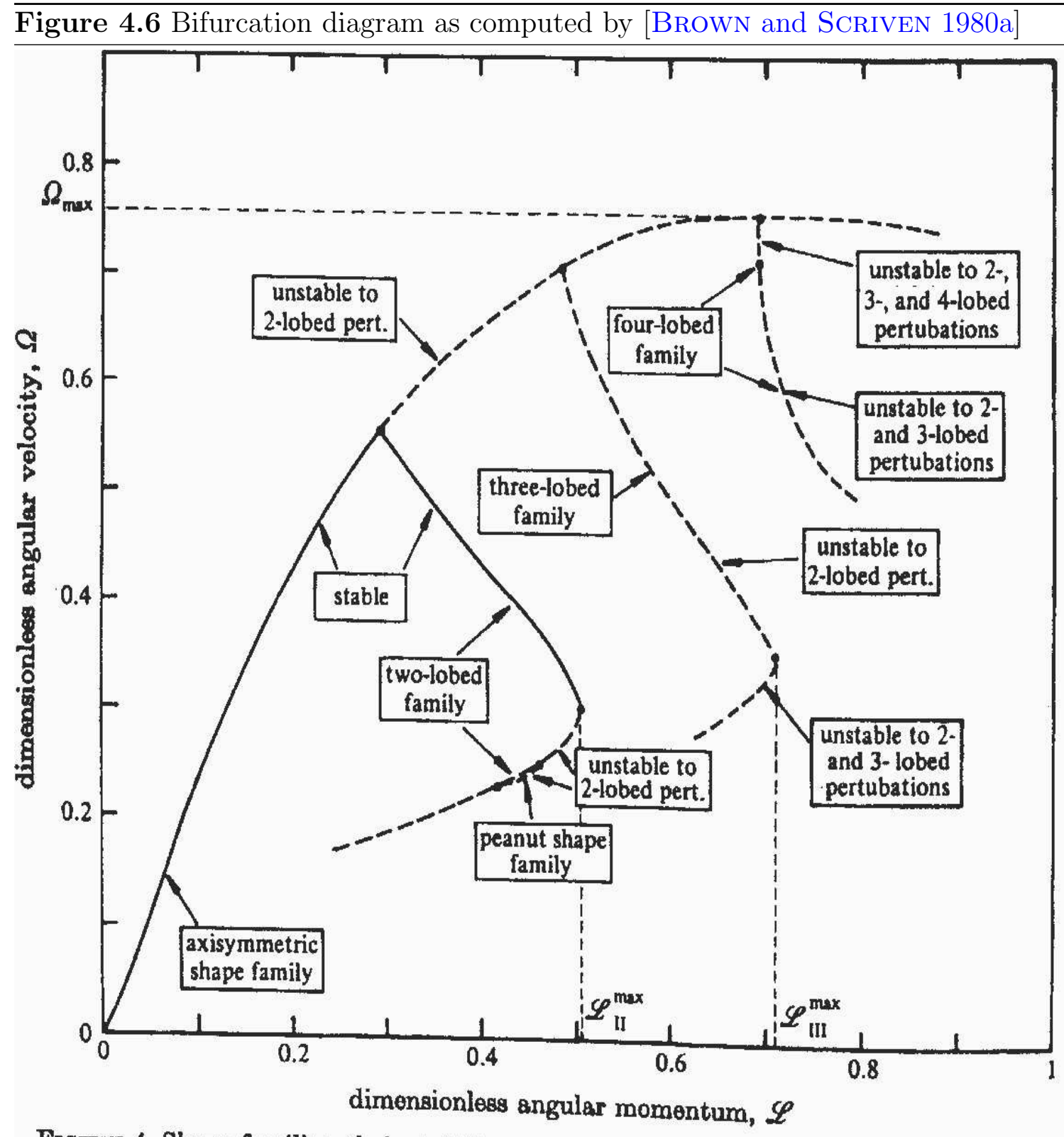

Figura 4. Shape families, their atability and bifurcetions, for drope isolated and so rotating at constant angular momentum. Shapes are stable along solid branches, unstable along 
We have chosen $\omega$ for the abscissa because it is the bifurcation parameter for our pathtracking algorithm.

We remind in this respect of the introductory Section 1.1 for the relation between the two variants of the energy functional which use either the angular velocity or the angular momentum as the bifurcation parameter.

The notion of an $n$-lobed drop shape used in Figure 4.6 on the facing page and Figure 4.7 will be made clear by the pictures on the following pages. The first difference between the two bifurcation diagrams is that Figure 4.7 shows many bifurcations not present in Figure 4.6. Besides the two-, three and four-lobed families already found by [Brown and Scriven 1980a] there are a five- and a six-lobed branch which bifurcate directly from the axially symmetric family. All families bifurcating from the axially symmetric family also have sub-branches (though Figure 4.7 doesn't show it for the five-lobed branch).

The major reason why we were able to find discrete solutions not found by Brown and Scriven is that they used a global parametrisation to represent the drop shapes. Another reason is that they were imposing an artificial meridional reflective symmetry.

Another difference between Figure 4.6 and 4.7 is that the scales of the axes do not match. The reason is that Brown and Scriven did not compensate for dissecting the drop at its equatorial and the assumed meridional symmetry-plane. This means that their angular momentum differs from ours by a factor of 4 .

Figure 4.7 Bifurcation diagram resulting from Algorithm 2.6.1 on page 58

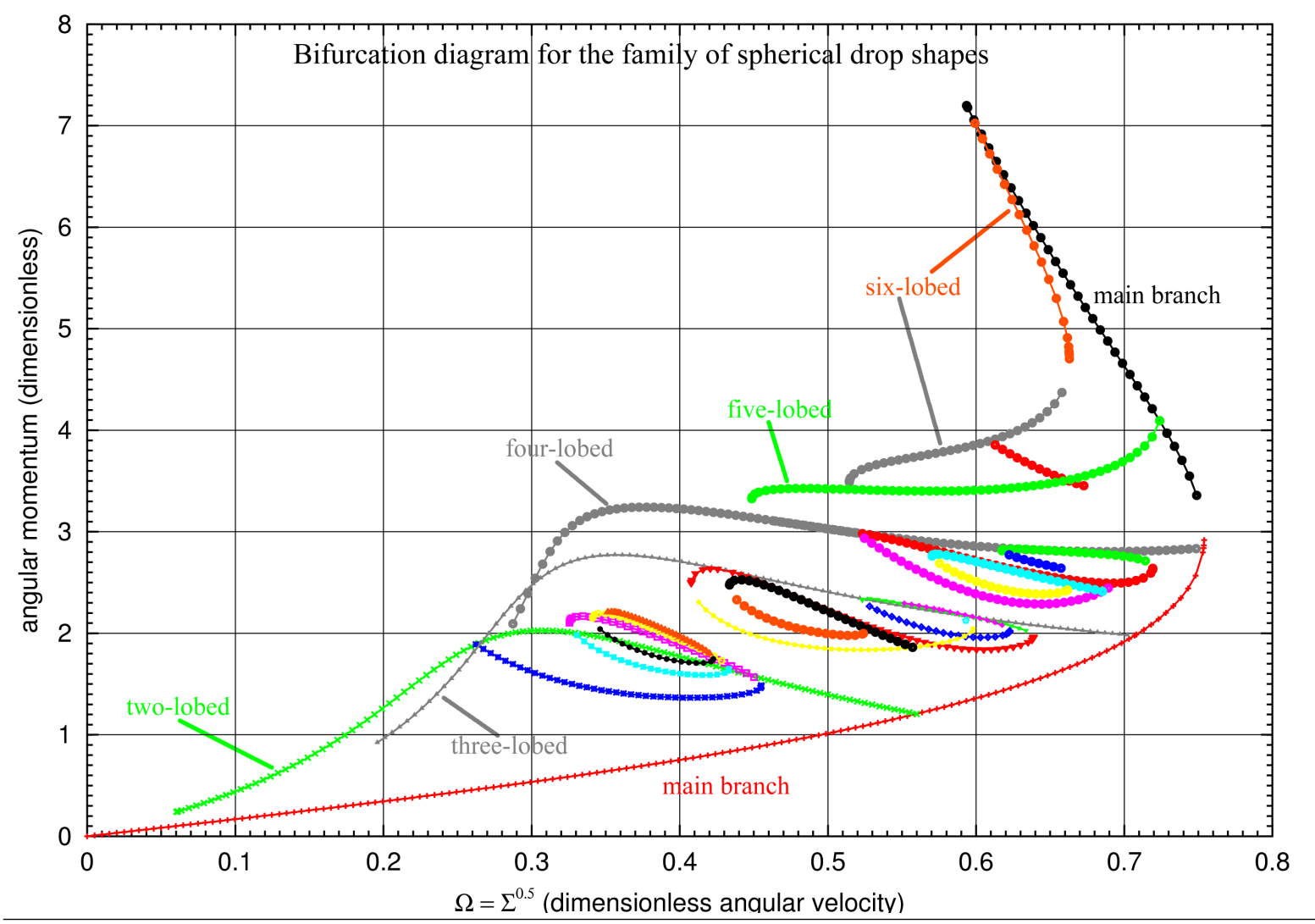


Figure 4.7 on the preceding page does not contain all the bifurcations we have found. We will present more detailed diagrams below which concentrate on certain parts of the solution family rather than presenting an overview over the entire set of discrete solutions computed by our path-tracking algorithm.

We have the conjecture that beyond the six-lobed family there are no further bifurcations from the main branch. We believe so because the eigenvalues of the linearised EulerLagrange equation seem to approach zero when tracking down the main branch beyond the bifurcation point of the six-lobed family towards smaller values of $\omega$. Figure 4.8 shows the smallest few eigenvalues of the linearised Euler-Lagrange equations for the axially symmetric family of spheroidal solutions.

$\overline{\text { Figure 4.8 Spectrum of the linearised Euler-Lagrange equations for the axially sym- }}$ metric spheroidal family after the turning point

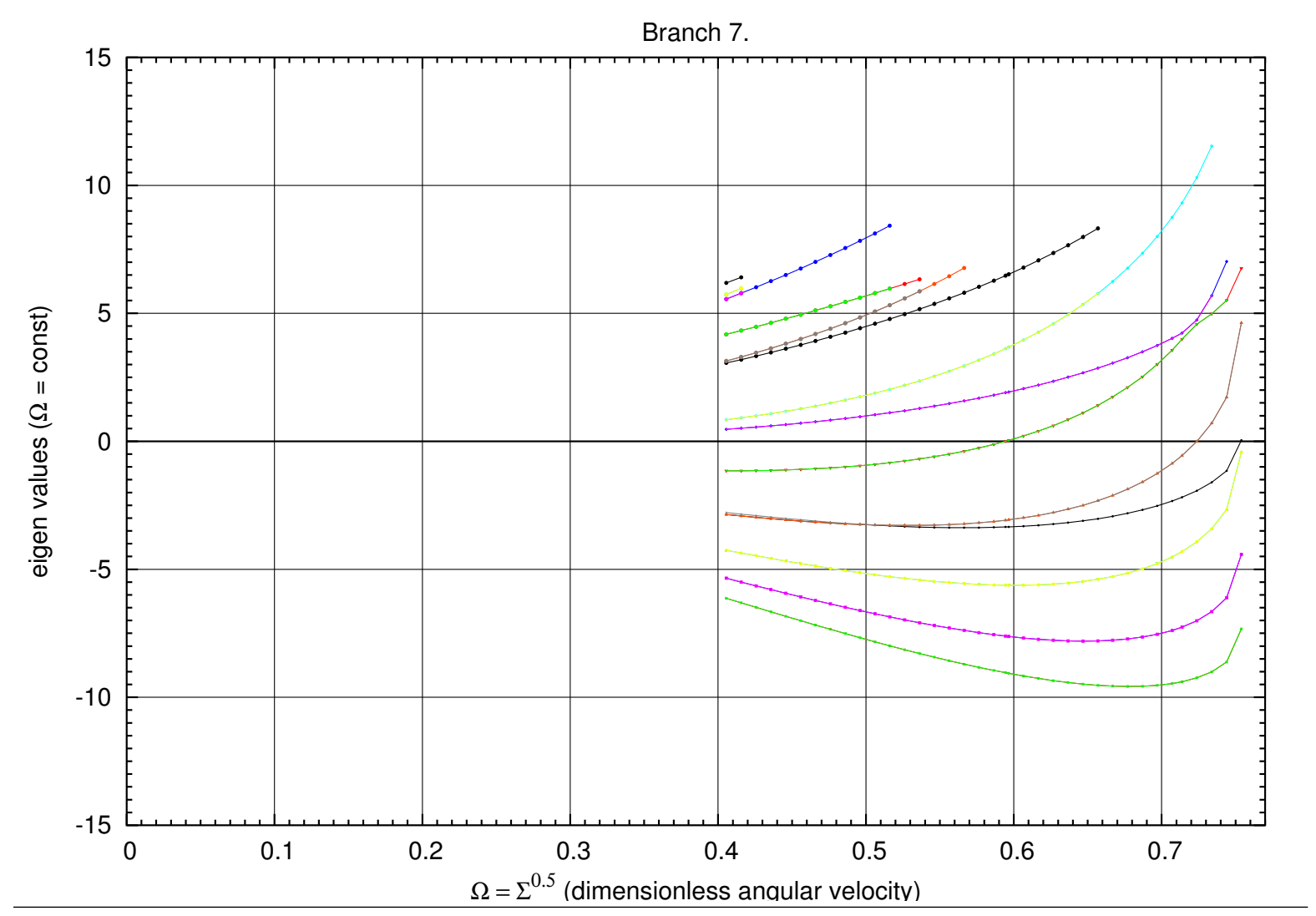

\subsubsection{Two-lobed spheroidal shapes}

The first bifurcation from the axially symmetric family of spheroidal drop shapes occurs at a value of $\omega \approx 0.5599$ which is in accordance with the value reported in [Brown and Scriven 1980a]. We note that [Wente 1982] has developed a method to estimate the actual value of $\omega$ to arbitrary accuracy. We note, however, that the value determined by the path-tracking method is inherently inexact because the linearization of the Euler-Lagrange equations becomes singular at a branch point which leads to numerical difficulties in the discretisation. 
Figure 4.9 shows a sequence of members of the two-lobed family of drop shapes. The two-lobed drops seem to approach a limiting surface consisting of two spheres touching each other in one point as $\omega$ approaches 0 .

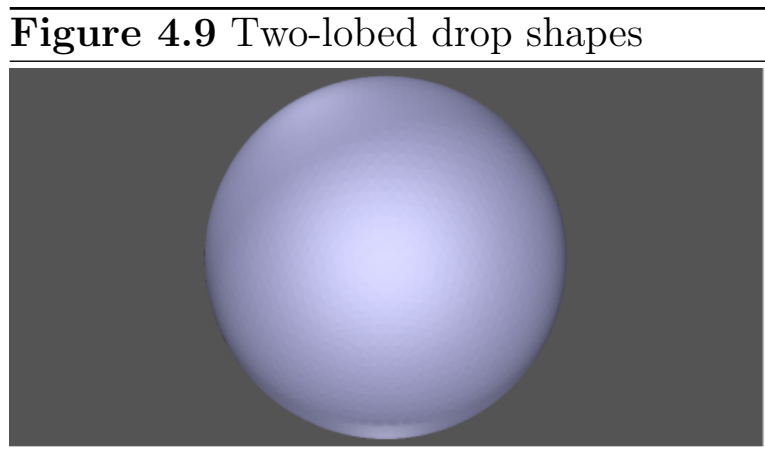

$\omega=0.5599$

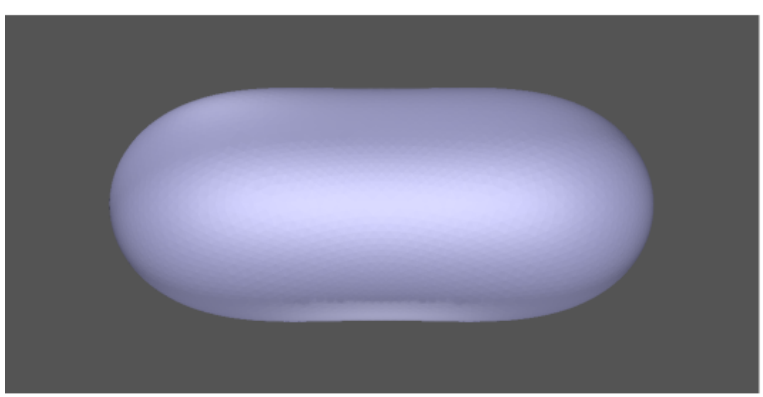

$\omega=0.4594$

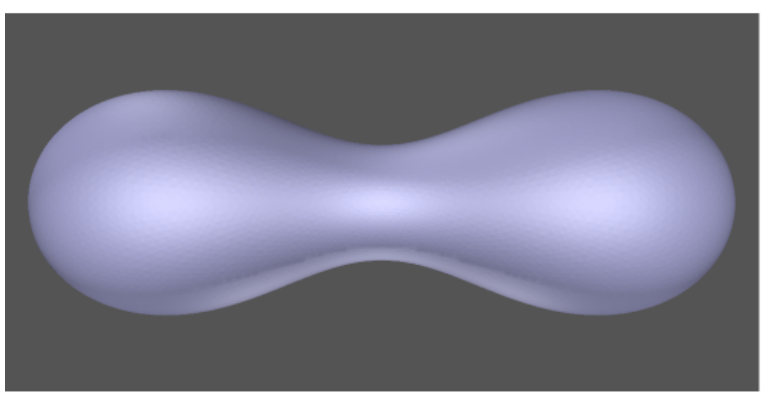

$\omega=0.2885$

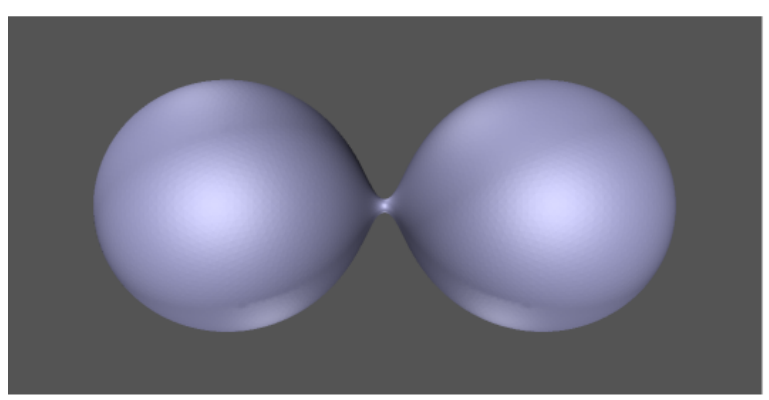

$\omega=0.1277$

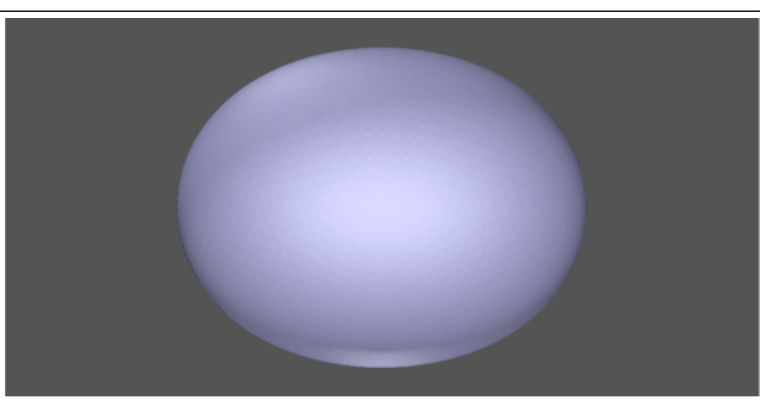

$\omega=0.5499$

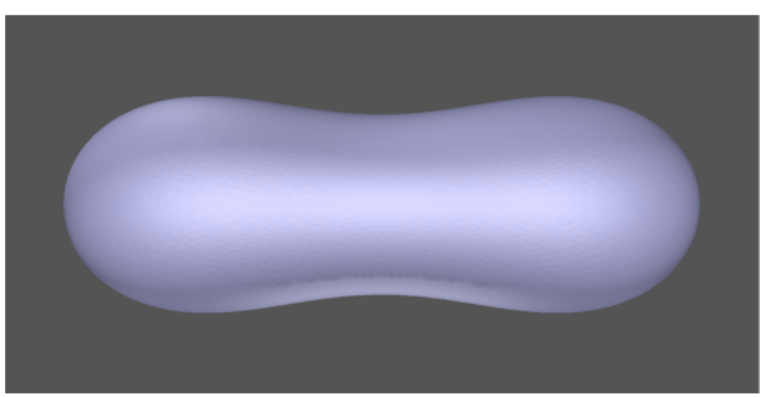

$\omega=0.3790$

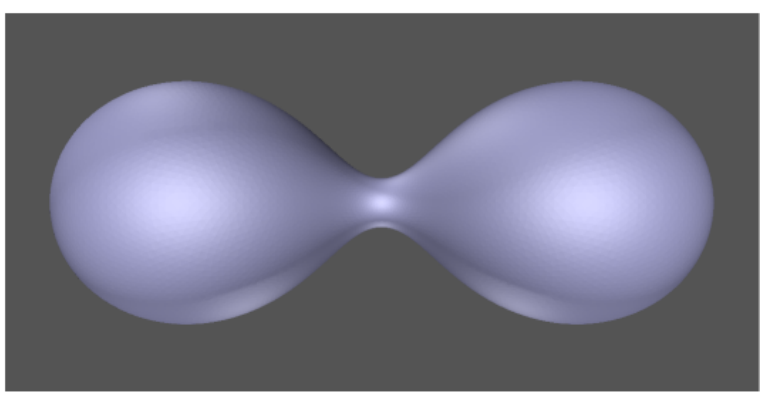

$\omega=0.2081$

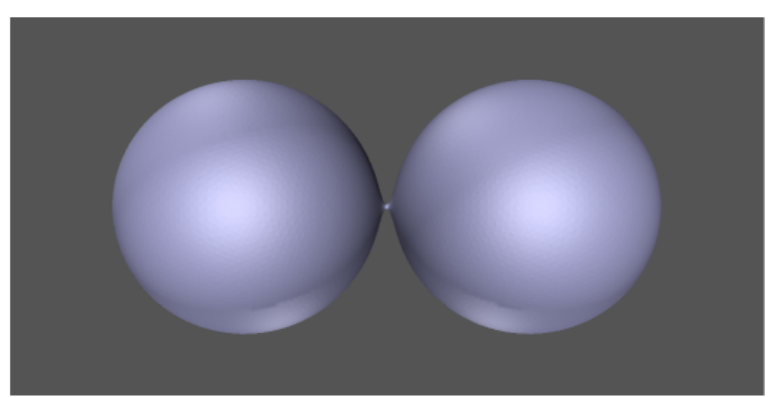

$\omega=0.0780$

The value of $\omega$ which marks the bifurcation point for the two-lobed family is important because it marks the onset of instability for the axially symmetric spheroidal family. Beyond this point the linearization has negative eigenvalues - regardless whether one imposes the additional constraint of conservation of angular momentum. This means 
that all spheroidal shapes for larger values of $\omega$ are (presumably) only saddle-points of the underlying energy functional.

It is remarkable in this respect that the first part of the two-lobed family might be stable if one imposes the constraint of conservation of angular momentum. Figure 4.10 shows that at the beginning of the two-lobed branch the (discretised) linearization has only positive eigenvalues when the angular momentum is conserved by the admissible perturbations. This was already found out by Brown and Scriven.

Figure 4.10 Spectrum of the linearised Euler-Lagrange equations for the two-lobed branch under the constraint of conserver angular momentum. The strange transversal lines at the right of the spectrum are just an artifact of the numerical construction of the initial value for the Newton iteration: the eigenvalue leading off to a new branch changes it multiplicity at the bifurcation point.

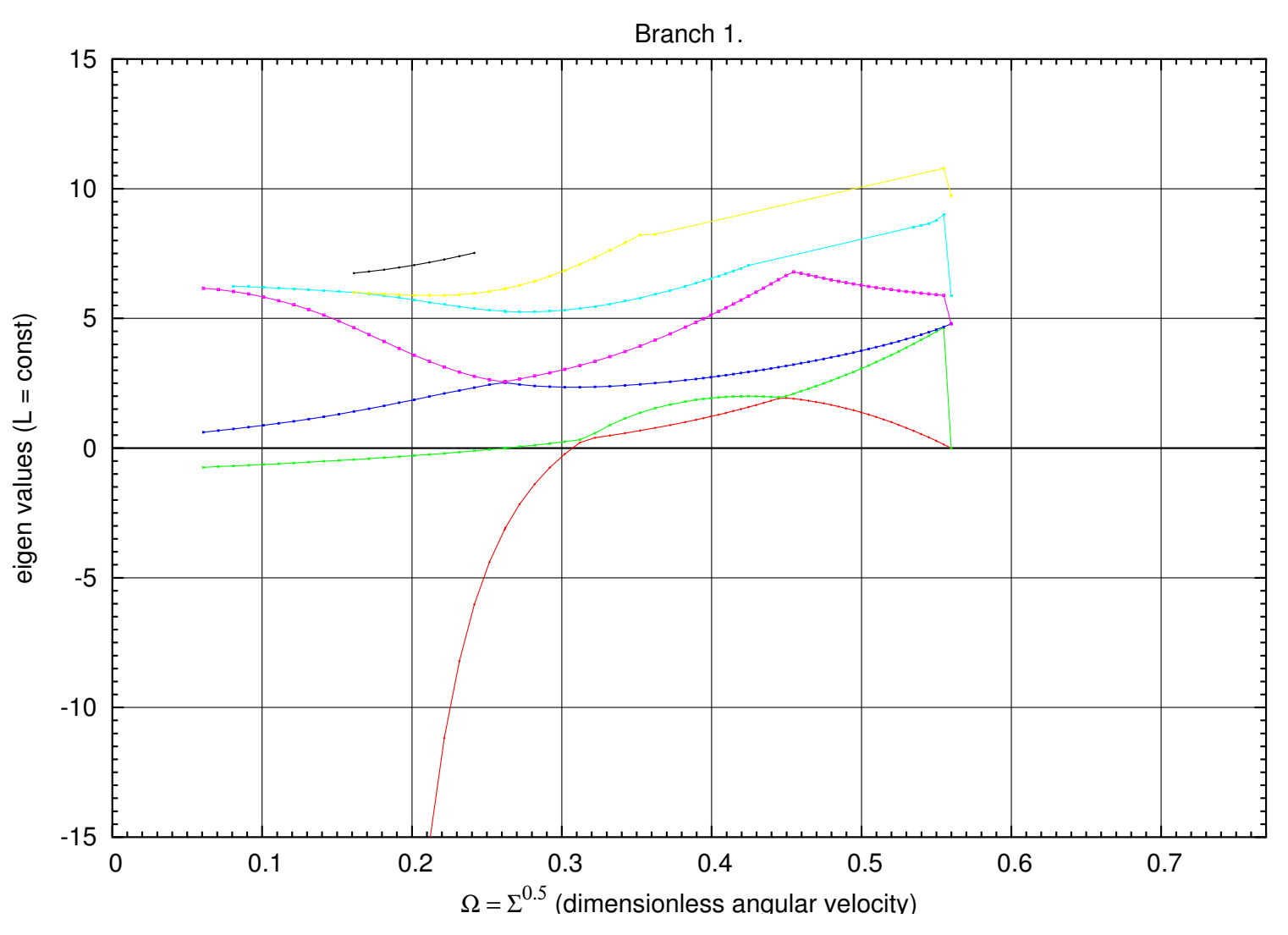

The two-lobed family has an interesting sub-branch which starts at $\omega \approx 0.262$. The form of the drops is illustrated in Figure 4.11 on the facing page. Brown and Scriven also found a sub-branch of similar shape at $\omega \approx 0.255$. However, they reported that the so called "peanut-shaped" branch reconnects to the two-lobed branch at a smaller value of the angular velocity. We could not reproduce their result. In fact, the branch of "peanut-shaped" discrete surfaces found by us branches off to larger values of $\omega$.

It is interesting that this new family of surfaces passes through several turning points and - up to the point where we terminated our path-tracking algorithm - develops an additional constriction after each turnaround in the $\omega$ - $L$ diagram. Figure 4.12 and 4.13 
on page 106 and 107 illustrate the "development" of the constrictions in greater detail (this is not a development in time; all surfaces are equilibrium figures).

We have the conjecture that this sub-branch of the two-lobed family develops arbitrary many constrictions while "winding up" itself in the $(\omega, L)$-plane.

We will see below that a similar behaviour seems to be typical for all sub-branches of the three- and four-lobed families and for the five- and six-lobed family bifurcating from the main branch.

Figure 4.11 Sub-branch of the two-lobed family branching at $\omega \approx 0.262$. The notion "Branch 1-2-3-4-5-6-7" indicates which eigenvalue was approaching 0 at each turning point, leading to the next turnaround.

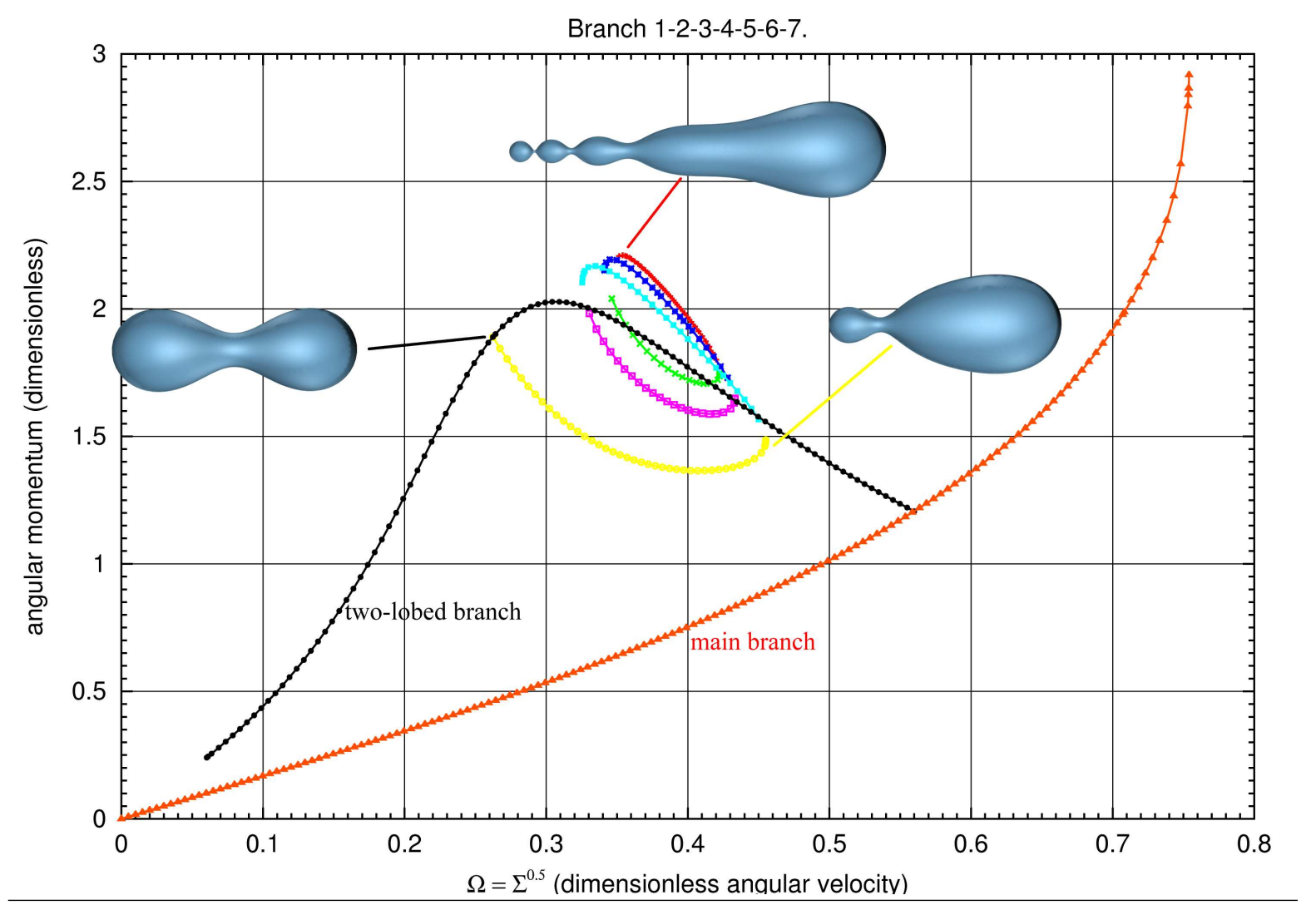


Figure 4.12 Two-lobed sub-branch, view from the top. The black cross marks the axis of rotation.

branch $1-2, \omega=0.2624$

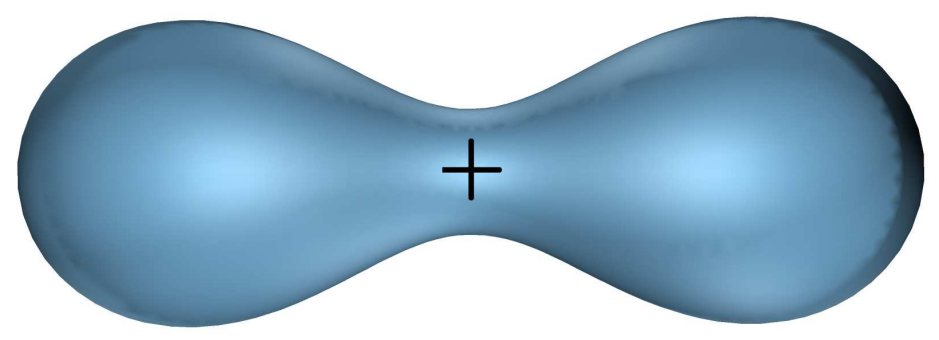

branch $1-2, \omega=0.3721$

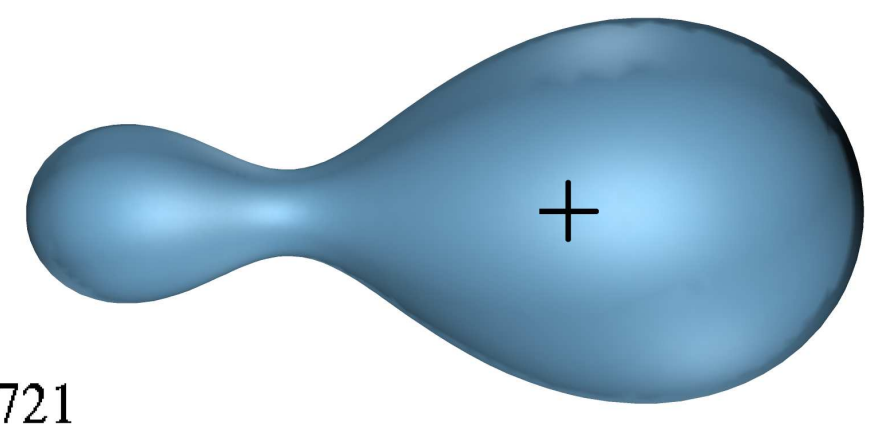

branch $1-2, \omega=0.4551$
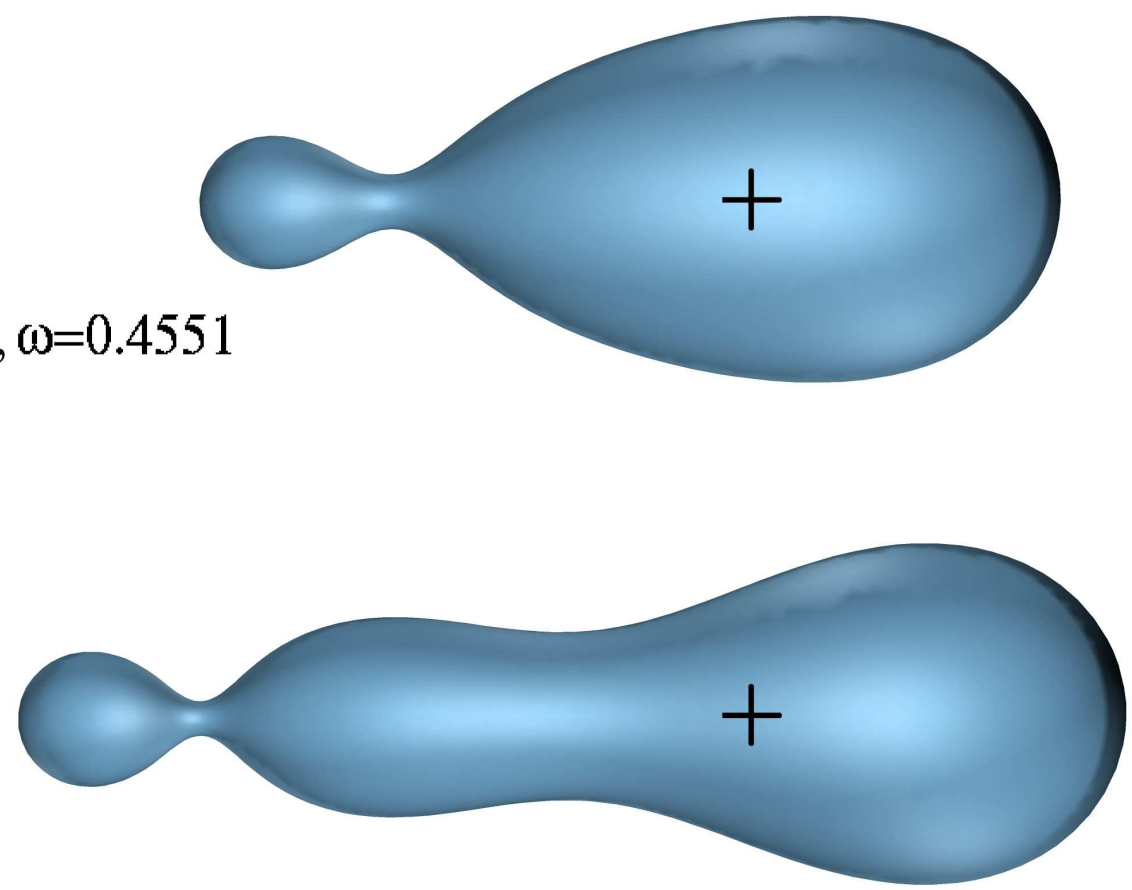

branch $1-2-3, \omega=0.3255$ 
Figure 4.13 Two-lobed sub-branch, continued from Figure 4.12 on the preceding page

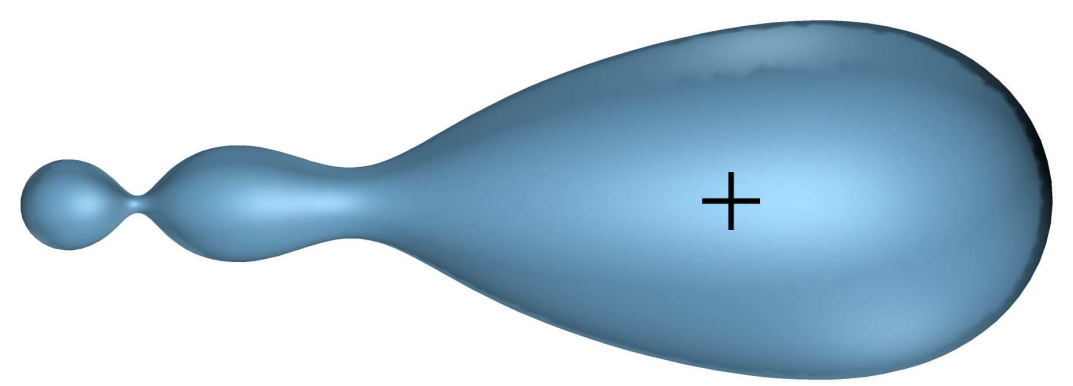

branch $1-2-3-4, \omega=0.4332$

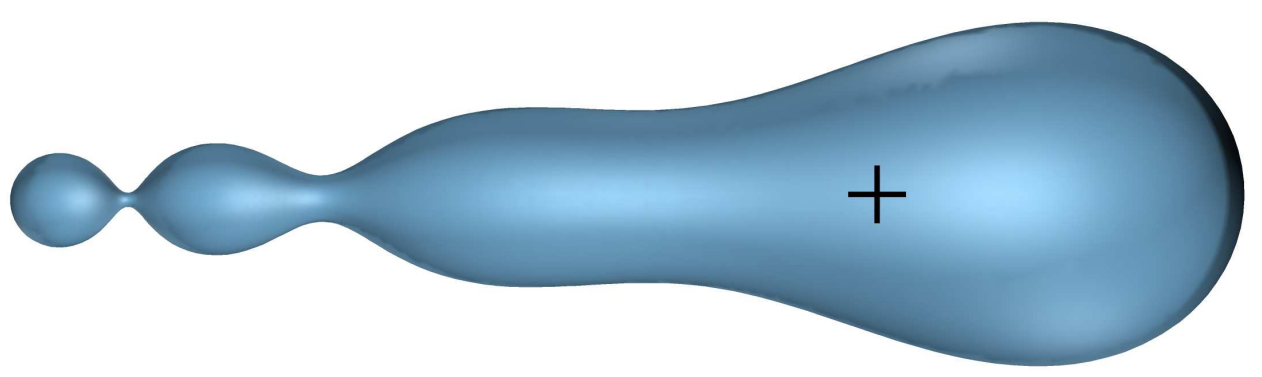

branch 1-2-3-4-5, $\omega=0.3413$

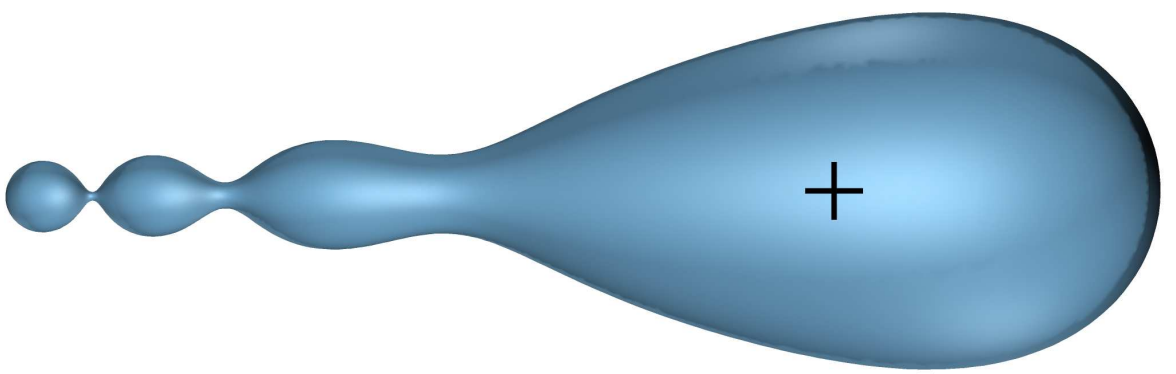

branch 1-2-3-4-5-6, $\omega=0.4226$

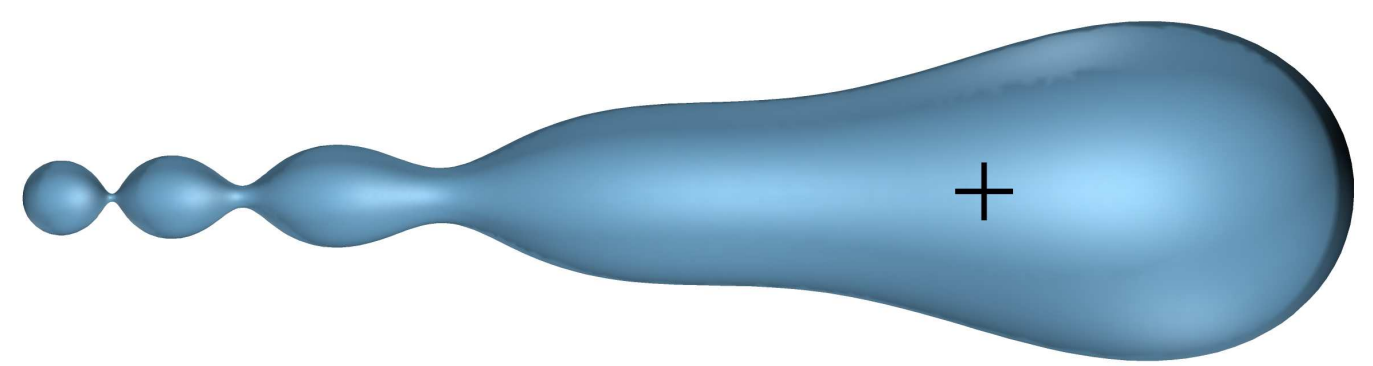

branch 1-2-3-4-5-6-7, $\quad \omega=0.3515$ 


\subsubsection{Three- and four-lobed shapes}

Like the two-lobed branch the three- and four-lobed branches seem to approach a limit surface consisting of spheres of equal size when $\omega$ tends to zero. Figure 4.14 illustrates this for the case of the three-lobed branch.

$\overline{\text { Figure 4.14 Three-lobed branch at } \omega=0.1056 \text {. This figure also illustrates the effectiv- }}$ ity of the mesh-smoothing algorithm and the concept of using a local parametrisation over the reference simplex which allows for surfaces with self-intersection.
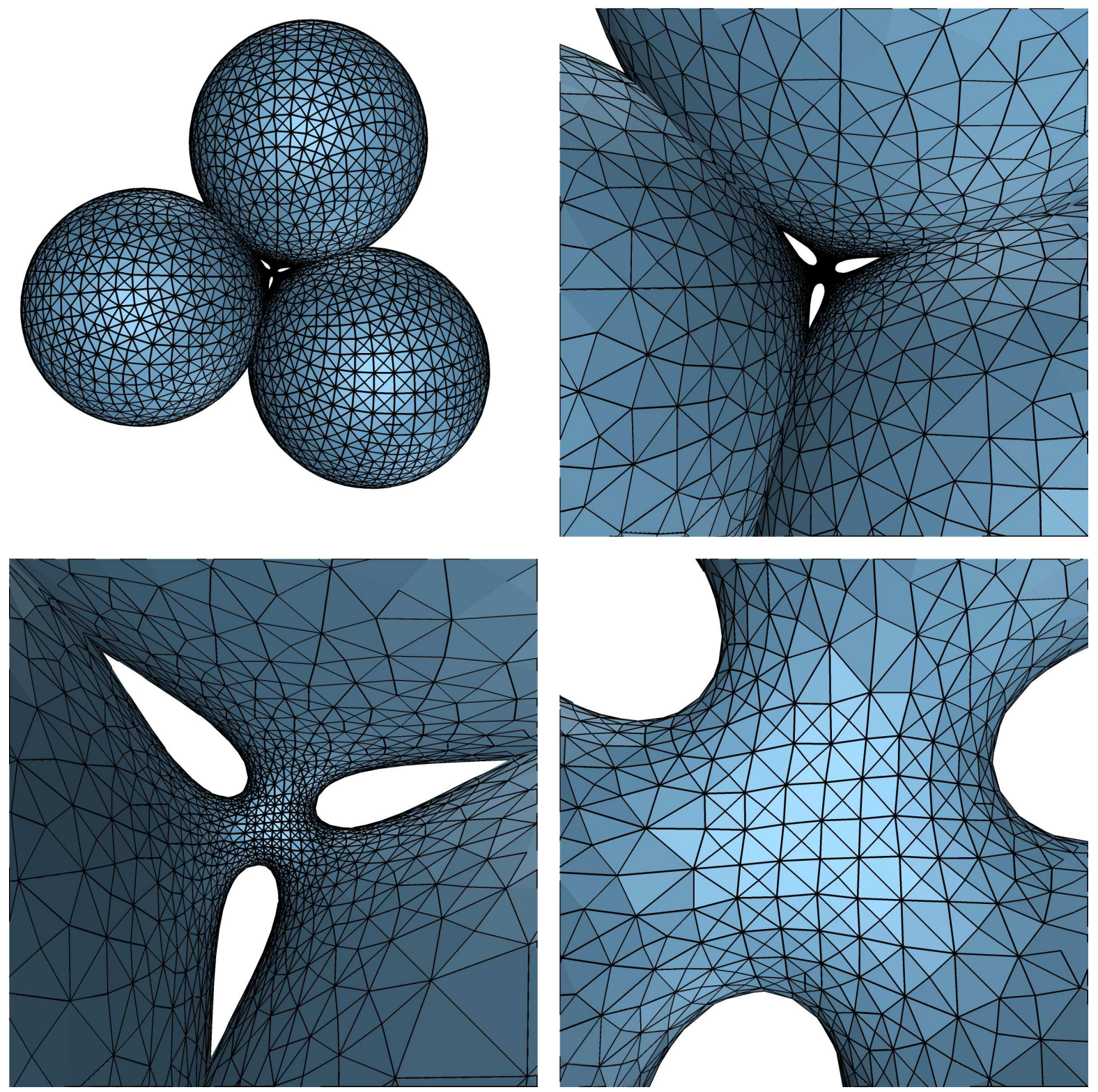

More interesting is the behaviour of the sub-branch which bifurcates from the three-lobed family at $\omega \approx 0.42$, see Figure 4.15 on the next page. First we note that the sub-branch leads to both, smaller and larger values of $\omega$. It seems to be tangential to the three-lobed branch in the $(\omega, L)$-plane. The sub-branch shows again the "winding-up" behaviour 
Figure 4.15 Three-lobed sub-branch breaking the meridional reflective symmetry

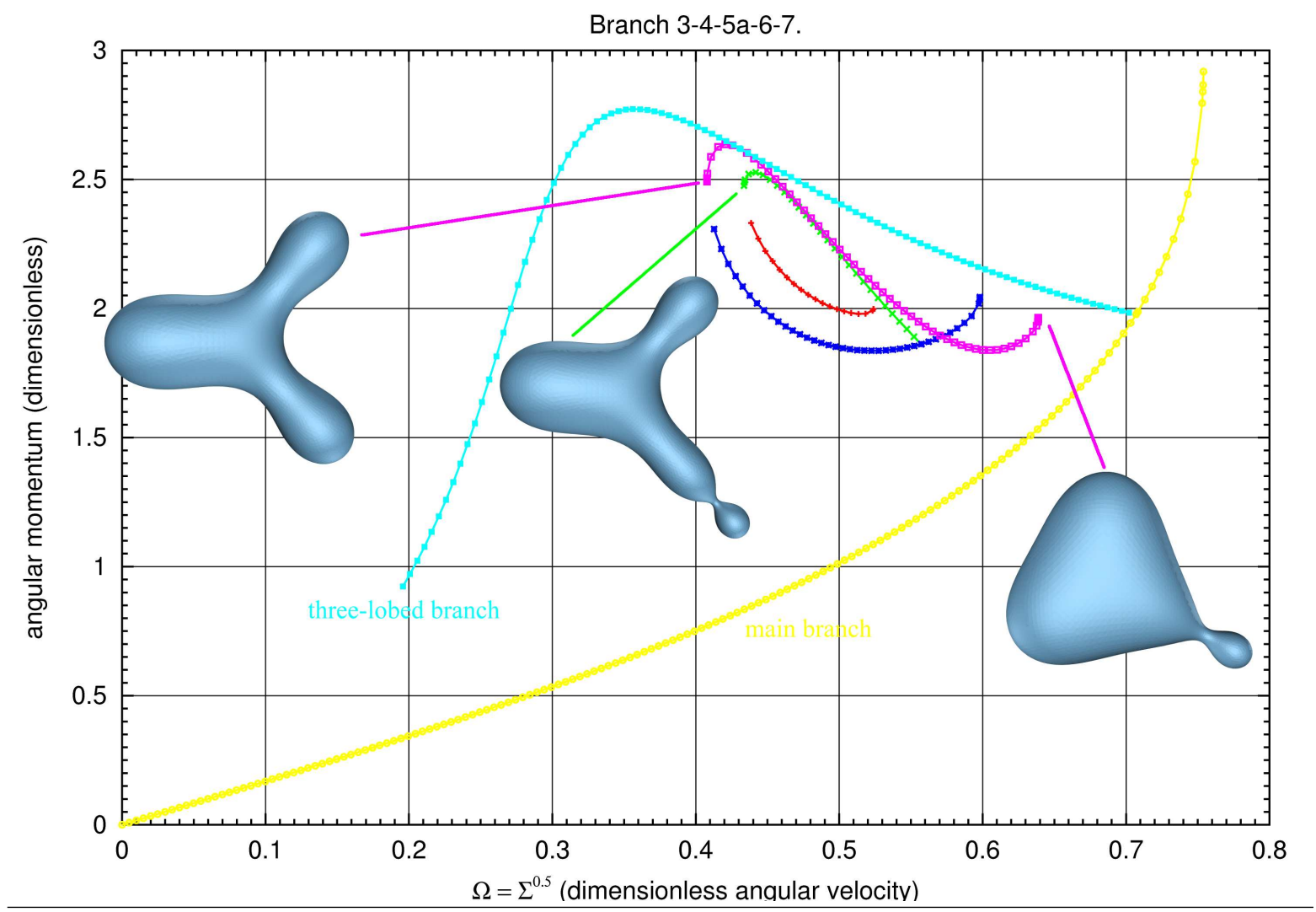

similar to the sub-branch of the two-lobed family, and it is highly probable that it continues beyond the point where we stopped our computations. However, the important point is that it develops a sub-sub-branch which breaks the meridional reflective symmetry.

The way this happens seems to be typical for the sub-branches in the respect that not all lobes develop constrictions simultaneously but only some of them.

Concerning the four-lobed shapes we just remark that they exhibit a similar behaviour as the three-lobed families. They seem to approach a four-sphere limit when $\omega$ tends to zero. The bifurcation diagram Figure 4.7 on page 101 shows sub- and sub-sub-branches which are related to the geometrically simpler "parent" branches by shrunken or grown lobes and additional constrictions, just as it was the case for the three-lobed family. 


\subsubsection{Five- and six-lobed spheroidal shapes}

The five- and six-lobed bifurcations differ from the two-, three- and four-lobed variants. They do not approach a limit surface consisting of spheres of equal size, but instead wind-up in the $(\omega, L)$-phase-space, see Figure 4.16 . We are rather brief here and just present some pictures on the following pages.

Figure 4.16 "Winding-up" of the two-, five- and six-lobed families

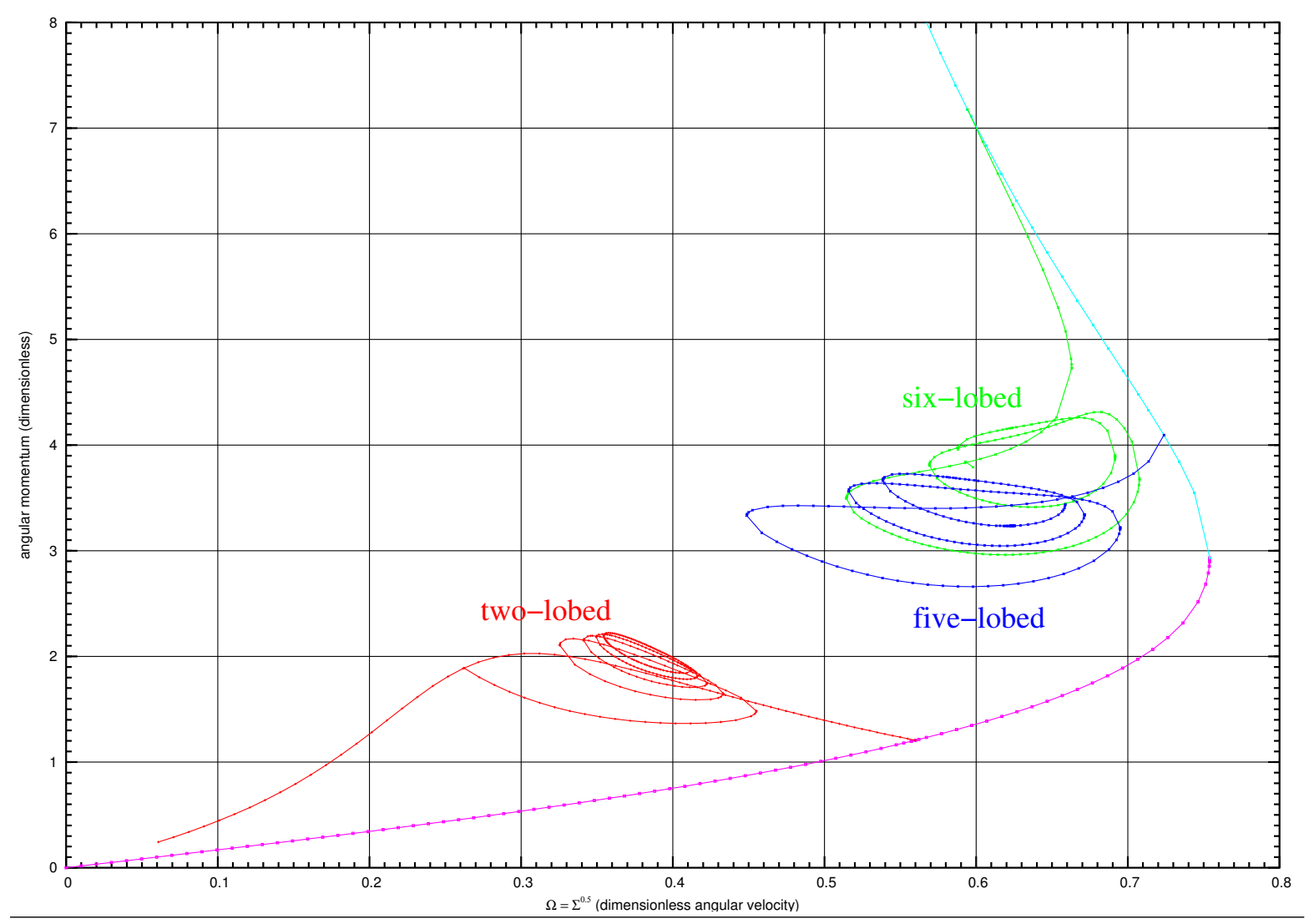




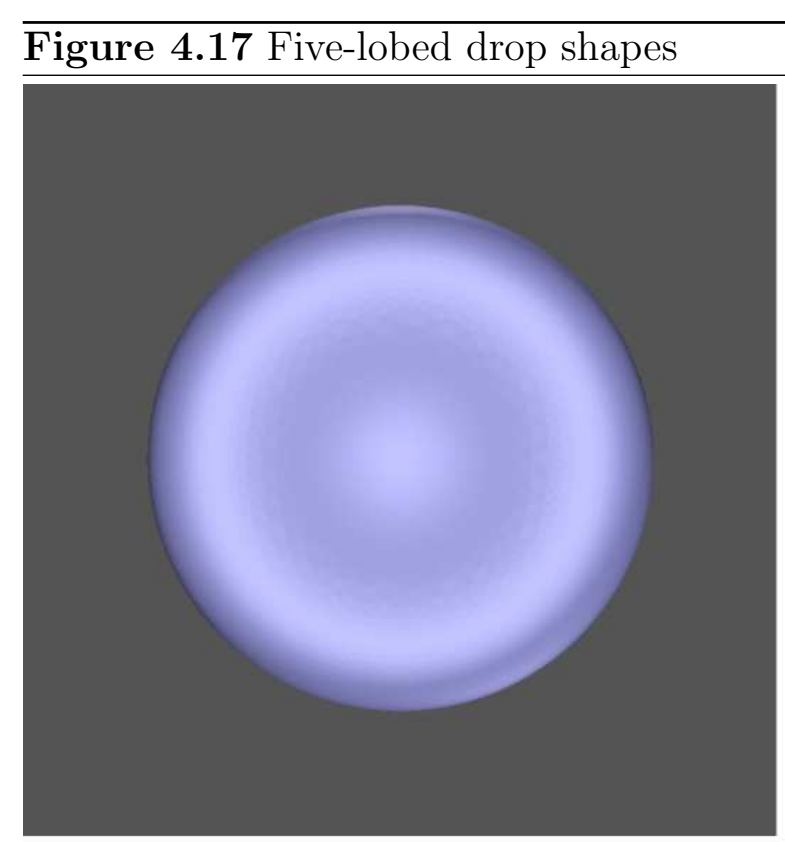

$\omega=0.7239$

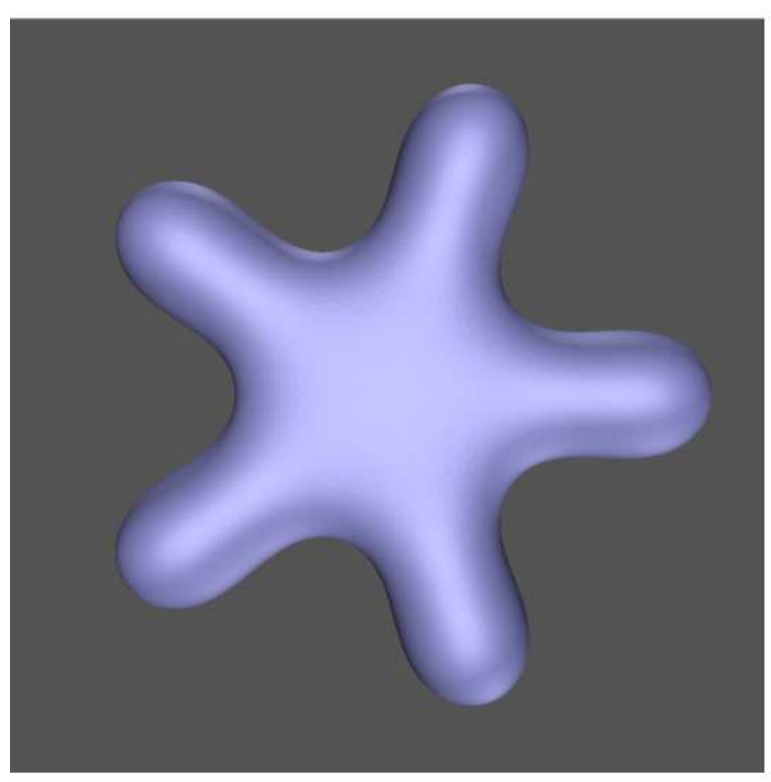

$\omega=0.5731$

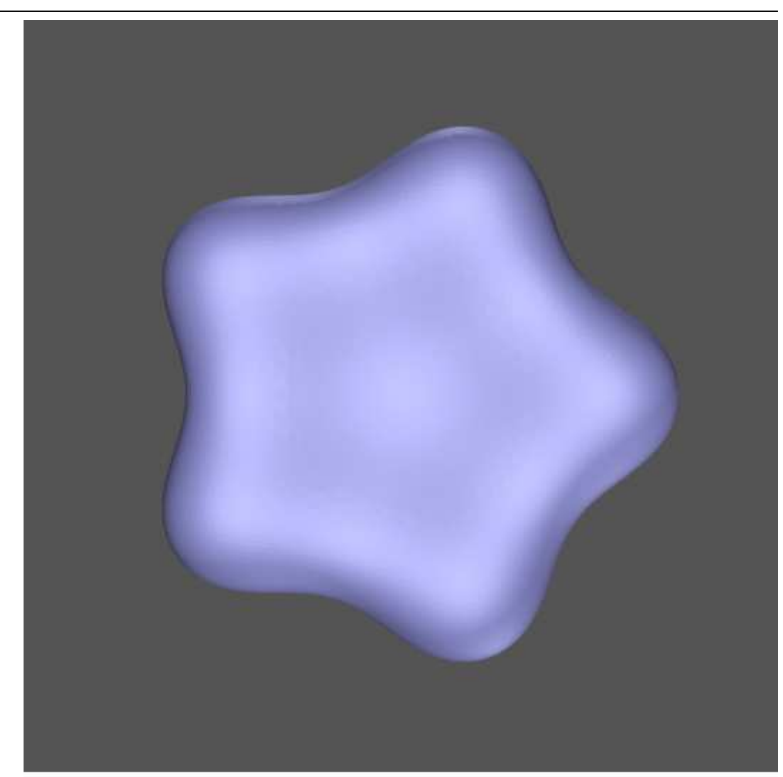

$\omega=0.7138$

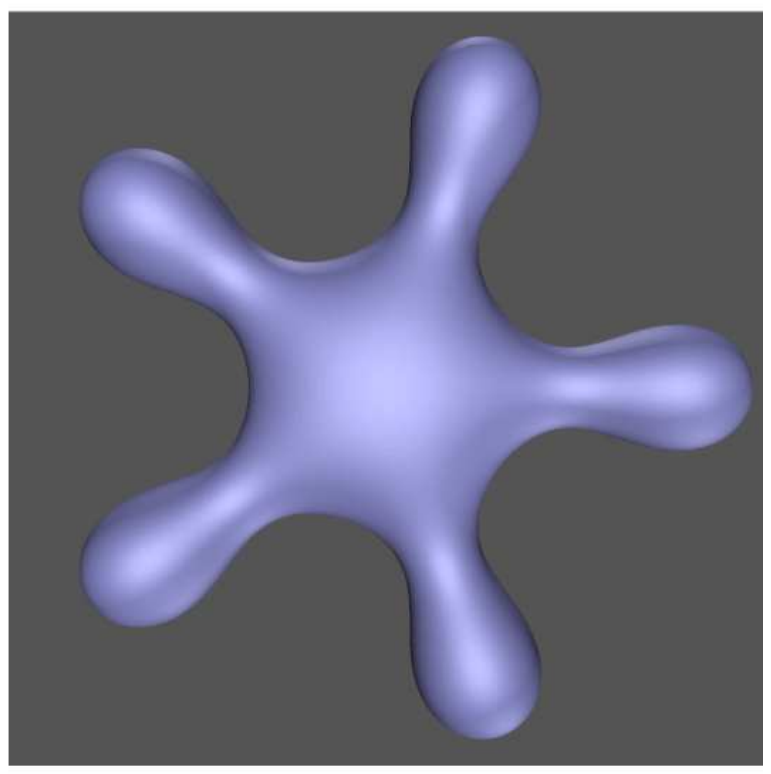

$\omega=0.4491$ 

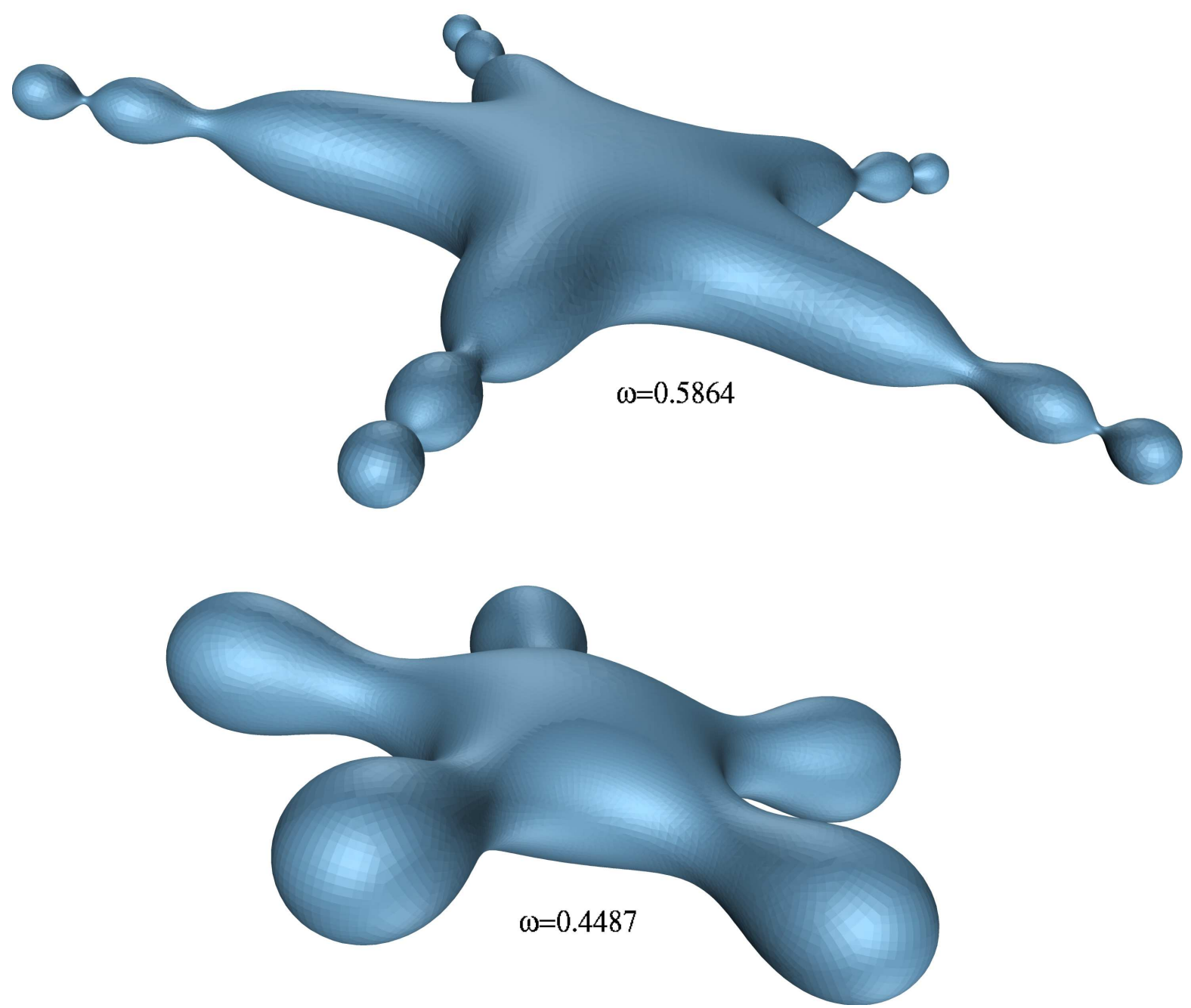
Figure 4.19 Members of the six-lobed branch shown in the bifurcation diagram Figure 4.7 on page 101. The bottom-right shape belongs to the sub-branch.
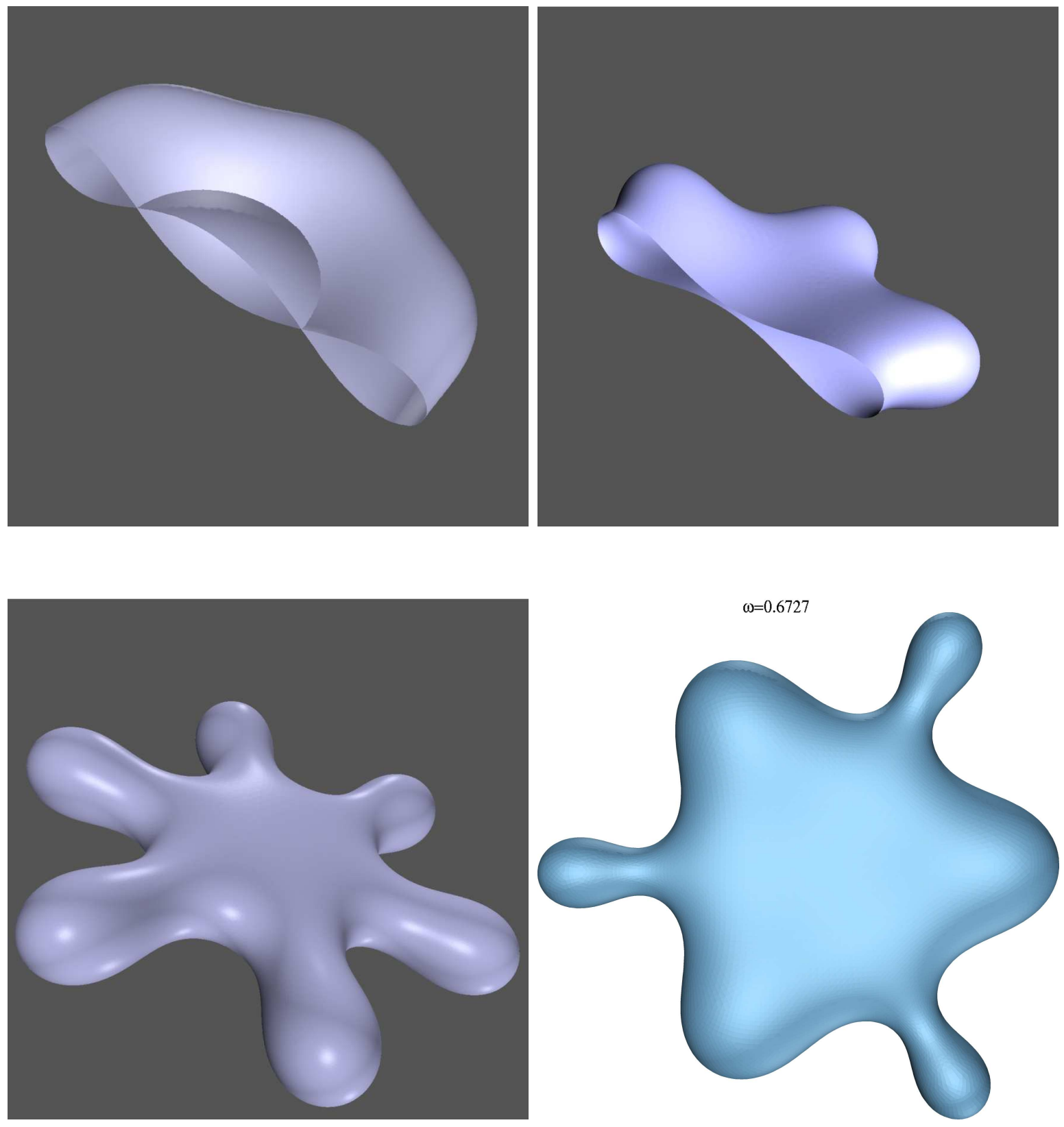


\subsubsection{Annular drop shapes}

The results of our computations with respects to bifurcations from the axially symmetric annular shapes are summarised in the bifurcation diagram shown in Figure 4.20.

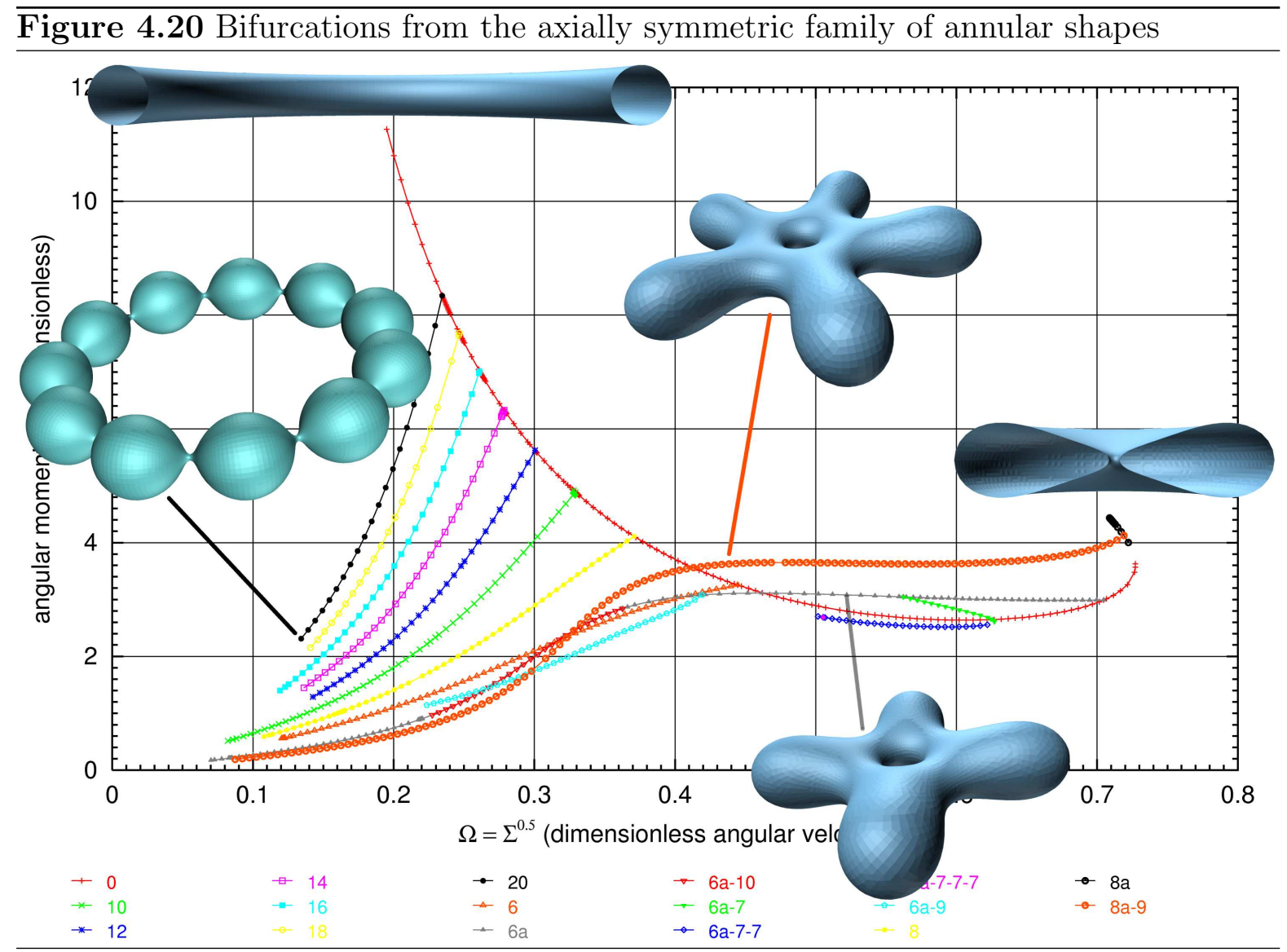

We remark that the "pearl-necklet"-like shape does not seem to be the final bifurcation of this kind. We have the conjecture that for decreasing $\omega$ there is a countable number of bifurcations, each one incrementing the number of constriction by 1 with respect to its predecessor. 


\section{Bibliography}

[Adams 1975] Adams, Robert A. (1975). Sobolev spaces. Academic Press [A subsidiary of Harcourt Brace Jovanovich, Publishers], New York-London. Pure and Applied Mathematics, Vol. 65. 51

[Aubin 1982] Aubin, ThierRy (1982). Nonlinear Analysis on Manifolds. MongeAmpère Equations. Springer Verlag, Berlin, Heidelberg, New York. 9, 36, 55

[Balendran 1999] Balendran, B. (1999). A Direct Smoothing Method for Surface Meshes. In Proceedings 8th International Meshing Roundtable, South Lake Tahoe, California. http://www.andrew.cmu.edu/user/sowen/imr9.html. 72

[B̈̈NSCH 1991] BÄNsch, E. (1991). Local mesh refinement in 2 and 3 dimensions. IMPACT of Computing in Science and Engineering, 3:181-191. 69, 70

[B̈̈Nsch and Siebert 1995] BÄnsch, Eberhard and K. G. Siebert (1995). A Posteriori Error Estimation for Nonlinear Problems by Duality Techniques. Preprint 30, Universität Freiburg. 53

[Barrett et al. 1994] Barrett, R., M. Berry, T. F. Chan, J. Demmel, J. Donato, J. Dongarra, V. Eijkhout, R. Pozo, C. Romine and H. V. DER VORST (1994). Templates for the Solution of Linear Systems: Building Blocks for Iterative Methods, 2nd Edition. SIAM, Philadelphia, PA. http://www.netlib.org/templates/Templates.html. 52

[BEer 1869] BeER, A. (1869). Einleitung in die mathematische Theorie der Elasticität und Capillarität. A. Giesen Verlag, Leipzig. In German. 5, 6, 16, 17, 42

[Braess 1997] Braess, D. (1997). Finite Elemente. Springer, Berlin, Heidelberg, New York, 2 ed. 52, 116

[Brown and Scriven 1980a] Brown, R. A. and L. E. Scriven (1980a). The shape and stability of rotating liquid drops. Proc. Roy. Soc. London Ser. A, 371(1746):331357. See also [Brown and Scriven 1980b]. iv, v, 5, 31, 54, 56, 100, 101, 102

[Brown and Scriven 1980b] Brown, R. A. and L. E. Scriven (1980b). The shapes and stability of captive rotating drops. Philos. Trans. Roy. Soc. London Ser. A, 297(1429):51-79. 115 
[Brulois 1987] Brulois, F. (1987). The Limit of Stability of Axisymmetric Rotating Drops. In Variational methods for free surface interfaces (Menlo Park, Calif., 1985), pp. 145-153. Springer Verlag. 6

[Canann et al. 1998] Canann, Scott A., J. R. Tristano and M. L. Staten (1998). An Approach to Combined Laplacian and OptimizationBased Smoothing for Triangular, Quadrilateral and Quad-Dominant Meshes. In Proceedings 7th International Meshing Roundtable, Dearborn, $M I$. http://www.andrew.cmu.edu/user/sowen/imr7.html. 73, 81

[Carmo 1992] Carmo, M.P. Do (1992). Differentialgeometrie von Kurven und Flächen. Vieweg, Braunschweig. 9, 12

[Carmo 1993] Carmo, M.P. do (1993). Riemannian Geometry. Birkhäuser, Boston, Stuttgart. 9

[Chandrasekhar 1965] Chandrasekhar, S. (1965). The stability of a rotating liquid drop. Proceedings of the Royal Society, London, A 286:1-26. 5, 17

[Chow and Hale 1996] Chow, Shui-NeE and J. K. Hale (1996). Methods of Bifurcation Theory. Springer Verlag, New York, 2 ed. 14, 15, 16

[Ciarlet and Raviart 1972] Ciarlet, P.G. and P.-A. Raviart (1972). Interpolation theory over curved elements, with applications to finite element methods. Computer Methods appl. Mech. Engin., 1:217-249. 47

[DJidjev 2000] DJidjev, Hristo N. (2000). Force-Directed Methods for Smoothing Unstructured Triangular and Tetrahedral Meshes. In Proceedings 9th International Meshing Roundtable, New Orleans. http://www.andrew.cmu.edu/user/sowen/imr9.html. 74, 77

[DzIUK 1988] DzIUK, G. (1988). Finite elements for the Beltrami operator on arbitrary surfaces.. In Hildebrandt, S. and R. Leis, eds.: Partial Differential Equations 86 Calculus of Variations, no. 1357 in Lecture Notes in Mathematics, pp. 142-155. Springer Verlag. 116

[DzIUk 1991] DzIuk, G. (1991). An Algorithm for Evolutionary Surfaces. Numerische Mathematik, Springer Verlag, 58:603-611. The algorithm uses [DzIUK 1988]. iv, v, 42

[Erikson and Johnson 1991] Erikson, Kenneth and C. Johnson (1991). Adaptive Finite Element Methods for Parabolic Problems I: A Linear Model Problem. SIAM Journal on Numerical Analysis, 28(1):43-77. 53

[Evans 1998] Evans, Lawrence C. (1998). Partial Differential Equations, vol. 19 of Graduate Studies in Mathematics. American Mathematical Society. 9, 29

[Fletcher and Reeves 1964] Fletcher, R. and C. Reeves (1964). Function minimization by conjugate gradients. The Computer Journal, pp. 149-154. FYI: quoted literally in [BRAESS 1997], p. 193. 75 
[Gallot et al. 1993] Gallot, S., D. Hulin and J. Lafontaine (1993). Riemannian Geometry. Springer Verlag, 2 ed. 9

[Gilbarg and Trudinger 2001] Gilbarg, David and N. S. Trudinger (2001). Elliptic Partial Differential Equations of Second Order. Classics in Mathematics. Springer Verlag, Berlin, Heidelberg, 1998, 2nd ed. 9, 13

[Gulliver 1984] Gulliver, R. (1984). Tori of prescribed mean curvature and the rotating drop. In E.H.A. Gonzales, I. TAMAnini, ed.: Variational methods for equilibrium problems of fluids. (Meeting held in Trento, Italy, from June 20 to 25, 1983)), vol. 118 of Asterisque, pp. 167-179. Societe Mathematique de France. iv, v, $16,19,41$

[Hutchinson 1991] Hutchinson, J.H. (1991). Computing conformal maps and minimal surfaces. Proc. C.M.A., (26):140-161. 79

[Kalik and Wendland 1992] Kalik, K. and W. Wendland (1992). The Approximation of Closed Manifolds by Triangulated Manifolds and the Triangulation of Closed Manifolds. Computing, (47):255-275. 44

[LANDAu and Lifschitz 1990] Landau, L.D. and E. Lifschitz (1990). Mechanik, vol. I of Lehrbuch der theoretischen Physik. Akademie-Verlag, Berlin, 13 ed. German translation of the Russian textbook by P. Ziesche. 3

[Lehoucq et al. 1998] Lehoucq, R. B., D. C. Sorensen and C. Yang (1998). ARPACK users' guide. Software, Environments, and Tools. Society for Industrial and Applied Mathematics (SIAM), Philadelphia, PA. Solution of large-scale eigenvalue problems with implicitly restarted Arnoldi methods. 2, 56

[Mitchell 1989] Mitchell, W.F. (1989). A comparison of adaptive refinement techniques for elliptic problems. ACM Transactions on Mathematical Software, 15:326347. 71

[Myshkis et al. 1987] Myshkis, A.D., V. Babskit, N. Kopachevskit, L. SlobozhAnIN and A. Tzuptsov (1987). Low-Gravity Fluid Mechanics - Mathematical Theory of Capillary Phenomena. Springer Verlag, Berlin, Heildelberg, New York. 5

[Nitsche 1975] Nitsche, Johannes C. C. (1975). Vorlesungen über Minimalflächen, vol. 199 of Die Grundlehren der mathematischen Wissenschaften in Einzeldarstellungen. Springer, Berlin, Heidelberg. 22, 24

[Owen 1998] Owen, Steven J. (1998). A Survey of Unstructured Mesh Generation Technology. In Proceedings Yth International Meshing Roundtable, Dearborn, MI. http://www.andrew.cmu.edu/user/sowen/imr7.html. 78

[Paige and Saunders 1975] Paige, C.C and A. Saunders (1975). Solution of sparse indefinite systems of linear equations. SIAM Journal on Numerical Analysis, 12. 52

[Plateau 1843] Plateau, J.A.F. (1843). Mémoire sur les phénomènes que présente une masse liquide libre et soustraite à l'action de la pesanteur. Mémoires de l'Academy Bruxelles, 16:1-35. [PlateAu 1863] is the English translation. 5, 118 
[Plateau 1863] Plateau, J.A.F. (1863). Experimental and theoretical researches on the figures of equilibrium of a liquid mass withdrawn from the action of gravity. Annual Report of the Board of Regents of the Smithsionian Institution, Washington, D.C., pp. 270-285. Translation of [Plateau 1843]. 4, 5, 117

[RAyleigh 1914] Rayleigh, LoRD (1914). The euquilibrium of revolving liquid under capillary force. Philosophical Magazine. 5

[Schmidt 1993] Schmidt, A. (1993). Die Berechnung dreidimensionaler Dendriten mit Finiten Elementen. PhD thesis, Albert-Ludwigs-Universität Freiburg i. Br. 60, $62,71,75,77,79,81,88,89,90,91$

[Schmidt and Siebert 1998] Schmidt, A and K. Siebert (1998). ALBERT 0.2 Adaptive multiL evel finite element toolbox using $\mathbf{B}$ isectioning refinement and $\mathbf{E}$ rror control by $\mathbf{R}$ esidual Techniques. [Schmidt and Siebert 1999]. 2, 59, 118

[Schmidt and Siebert 1999] Schmidt, A. and K. Siebert (1999). Abstract data structures for a finite element package: Design principles of ALBERT. Zeitschrift für Angewandte Mathematik und Mechanik, (79):49-52. 118

[Schmidt and Siebert 2000] Schmidt, A. and K. Siebert (2000). ALBERT Manual. Manual for [SchmidT and SieBert 1998]. 69

[Slobozhanin 1968] Slobozhanin, L.A. (1968). Hydrostatics in weak force fields. On the annular equilibrium shapes of a rotating liquid and on their stability. Izv. Akad. Nauk. SSSR, Mehk. Zhid. Gaza, 69(4). In Russian. 5

[Thomée 1980] ThomÉE, V. (1980). Galerkin finite element methods for parabolic problems. No. 1054 in Lecture Notes in Mathematics. Springer. 60

[Verfürth 1994] Verfürth, R. (1994). A posteriori error estimates for nonlinear problems. Finite element discretisations of elliptic problems.. Mathematics of Computation, 62:445-475. 53

[Wang 1988] Wang, T.G. (1988). Equilibrium Shapes of Rotating Spheroids and Drop Shape Oscillations. In Hutchinson, J.W. and T. Wu, eds.: Advances in Applied Mechanics, vol. 26, pp. 1-62. Academic Pres, Inc. 5, 6

[Wente 1982] Wente, H.C. (1982). The Symmetry of Rotating Fluid Bodies. Manuscripta Mathematica, Springer Verlag, 39:287-296. 5, 30, 102

[Wloka 1982] Wloka, Joseph (1982). Partielle Differentialgleichungen. B.G. Teubner, Stuttgart. 9, 14

[Zhou and Shimada 2000] Zhou, Tian and K. Shimada (2000). An Angle-Based Approach to Two-Dimensional Mesh Smoothing. In Proceedings 9th International Meshing Roundtable, New Orleans. http://www.andrew.cmu.edu/user/sowen/imr9.html. 78, 79 


\section{Index of Notations}

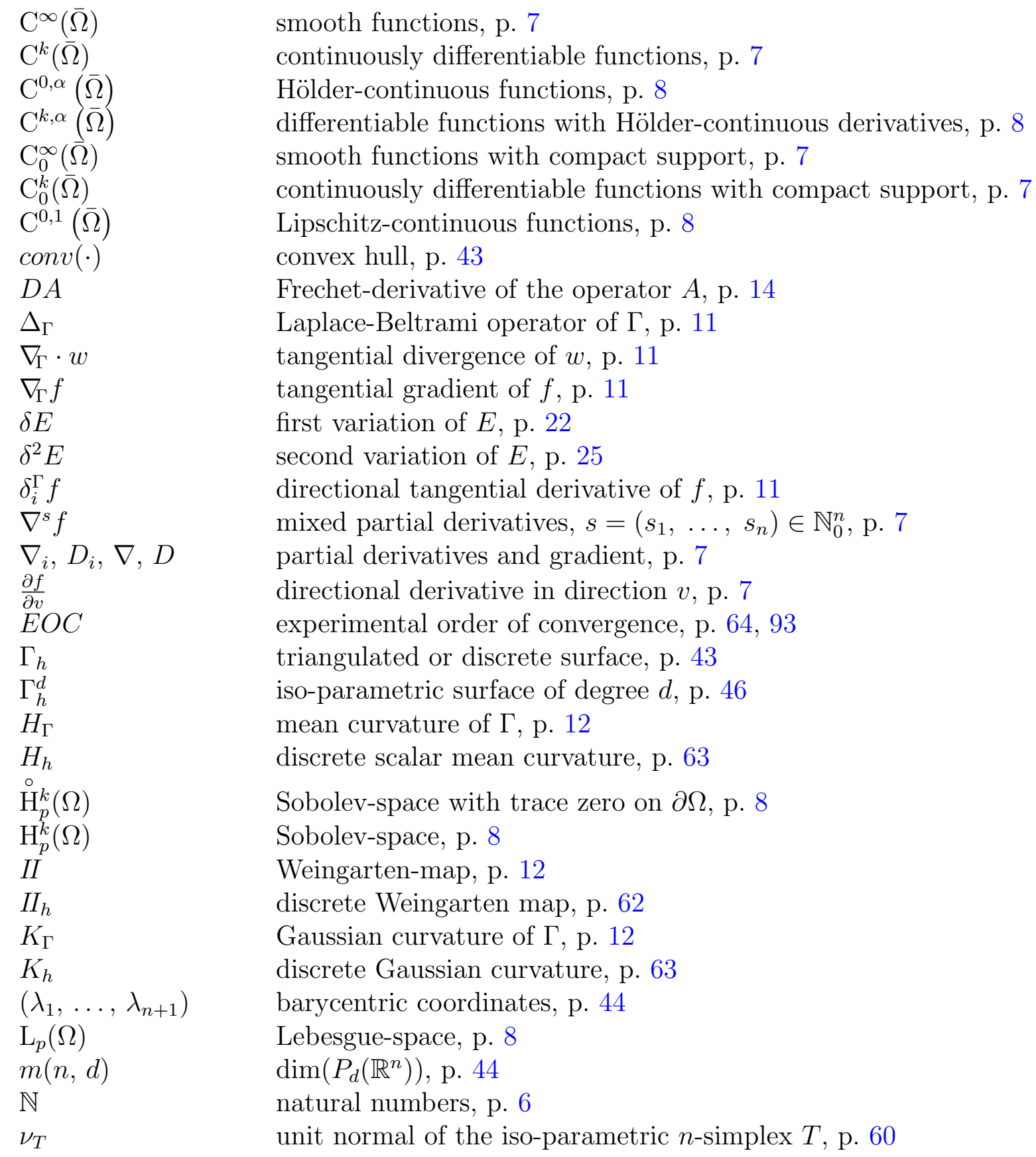


$\nu_{h}$

$\Phi_{T}^{d}$

$P_{k}$

$\Phi *$.

$\hat{p}_{j}$

$\mathbb{R}$

$\mathbb{R}^{+}$

$S(P)$

$\hat{S}(P)$

$A: B$

$x \cdot y,\langle x, y\rangle$

$T \Gamma$

$T_{P} \Gamma$

$\mathcal{T}_{h}$

$\mathcal{T}_{h}^{d}$

$\hat{T}$

$W_{h}^{d}\left(\Gamma_{h}^{d}\right)$

$Y_{\Gamma}$

$Y_{h}$ approximate discrete unit normal field, p. 60

polynomial parametrisation of degree $d$ over $\hat{T}$, p. 46

polynomials over $\mathbb{R}^{n}$ of degree at most $k$, p. 7

pull back w.r.t. to $\Phi$, p. 41

$j^{\text {th }}$-Lagrange polynomial, p. 44

real numbers, p. 6

positive real numbers, p. 6

the "star" of $P$, the elements adjacent to the vertex, p. 60, 72

projected element neighbourhood, p. 75

Euclidean scalar product between matrices, p. 6

Euclidean scalar product, p. 6

tangent bundle, p. 11

tangent space of $\Gamma$ at $P$, p. 10

triangulation, p. 43

iso-parametric triangulation of degree $d$, p. 46

reference simplex, p. 44

finite element space over $\Gamma_{h}^{d}$, p. 47

curvature vector, p. 12

discrete curvature vector, p. 62 


\section{Curriculum vitae}

Name: $\quad$ Claus-Justus Heine

Geburtsdatum: 8. Mai 1969

Geburtsort: Krefeld

\section{Bildungsgang}

1975-1979 Besuch der katholischen Grundschule Sollbrüggenstraße, Krefeld

1979-1988 Besuch des Gymnasiums Fabritianum, Krefeld

28.5.1988 Erwerb der allgemeinen Hochschulreife

10/1990-12/1997 Student an der RWTH Aachen

9/1992 Vordiplom in Mathematik und Physik

22.12.1997 Diplom in Mathematik

\section{Berufliche und ehrenamtliche Tätigkeiten}

4.7.1988-28.2.1990 Zivildienst am Krankenhaus St. Josefshospital Uerdingen, Krefeld 1991-1997 Beschäftigungen als studentische Hilfskraft (Kleingruppenübungen in Mathematik und Physik)

1995-1997 Beschäftigungen als studentische Hilfskraft für Computereinführungskurse im CIP-Pool der physikalischen Institute der RWTH Aachen

1/1998-2/2003 Wissenschaftlicher Angestellter des Instituts für Mathematik, RWTH Aachen. Betreuung von Übungen, Systemadministration (Unix/Windows), Forschungstätigkeit.

10/1997-10/2000 Organisatorische Leitung des Kammerensembles coll' arco

6/2000-6/2002 Vorstandsarbeit im Jungen Sinfonieorchester Aachen

seit 10/1996 Leitung des Ftape Projektes (Kerneltreiber für Floppystreamer) für das Betriebssystem Linux

3/2003-12/2003 Wissenschaftlicher Angestellter der Abteilung für Angewandte Mahtematik, Albert-Ludwigs-Universität Freiburg. Betreuung von Übungen, Forschungstätigkeit. 